\title{
WestVirginiaUniversity
}

THE RESEARCH REPOSITORY @ WVU

Graduate Theses, Dissertations, and Problem Reports

2014

\section{Effects of Aquatic Exercise on Executive Function in the Aging Population}

Lori A. Sherlock

West Virginia University

Follow this and additional works at: https://researchrepository.wvu.edu/etd

\section{Recommended Citation}

Sherlock, Lori A., "Effects of Aquatic Exercise on Executive Function in the Aging Population" (2014). Graduate Theses, Dissertations, and Problem Reports. 657.

https://researchrepository.wvu.edu/etd/657

This Dissertation is protected by copyright and/or related rights. It has been brought to you by the The Research Repository @ WVU with permission from the rights-holder(s). You are free to use this Dissertation in any way that is permitted by the copyright and related rights legislation that applies to your use. For other uses you must obtain permission from the rights-holder(s) directly, unless additional rights are indicated by a Creative Commons license in the record and/ or on the work itself. This Dissertation has been accepted for inclusion in WVU Graduate Theses, Dissertations, and Problem Reports collection by an authorized administrator of The Research Repository @ WVU.

For more information, please contact researchrepository@mail.wvu.edu. 
Effects of Aquatic Exercise on Executive Function in the Aging Population

Lori A. Sherlock

Dissertation submitted to

College of Human Resources and Education at

West Virginia University

In partial fulfillment of the requirements for the degree of

Doctor of Education

In Curriculum and Instruction

James Rye, Ph.D., Chair

W. Guyton Hornsby, Jr., Ph.D.

Sam Zizzi, Ed.D.

Mia Erickson, Ed.D.

Patricia Obenauf, Ph.D.

Department of Education Theory and Practice

Morgantown, West Virginia

2014

Key words: Aquatic Exercise, Water Exercise, Adult Learning, Aging, Older Adult, Executive Function, Exercise Barriers, Cognitive Function

Copyright 2014 Lori A. Sherlock 


\begin{abstract}
Effects of Aquatic Exercise on Executive Function in the Aging Population Lori A. Sherlock
\end{abstract}

Neurocognitive decline, including Alzheimer's Disease and other forms of dementia, is considered to be the world's fastest growing disease (Alzheimer's Association, 2011). Due to this escalation, research focused on determining causes, accelerants, impeding factors, and preventative strategies has become a focus of interest within the field. One of the principal points of study is the role that exercise plays in the maintenance or fortification against neurocognitive decline. Though there is a robust library of research focused on the effects of land-based exercise on cognitive function, currently there is no research that discusses the impact of aquatic-based exercise on these parameters.

This mixed method study focused on identifying the effects of a 10-week aquatic exercise intervention on parameters of executive function in individuals aged 60 years or older. Additional research questions targeted changes in cardiovascular fitness, wellness, and psychosocial barriers as well as behavior change in relation to the incorporation of adult education, and accessibility to exercise. Thirty-four volunteers between the ages of 60 and 90 years were recruited for this study. All participants took part in three preliminary visits consisting of assessment of physical parameters (anthropometric measurements, 6-minute walk test, RHR, RBP), psychosocial mediators (barriers, self-efficacy, motivators, social support), and cognitive variables (CANTAB ${ }^{\circledR}$ testing). Participants were then grouped into matched pairs and randomly assigned to the control or intervention group. The control group agreed to not alter their physical activity status while the intervention group took part in a 10-week aquatic exercise 
program employing progressive overload and adult education concepts. At the conclusion of the intervention, all participants $(\mathrm{n}=34)$ returned for their physiological, psychosocial, and cognitive post assessments. In addition to the post-testing, the intervention group also participated in a focus group to provide the researcher with qualitative data to associate with the quantitative findings.

Following data collection, a 2-way ANOVA with a group (treatment versus control) by time (pre and post intervention) was utilized to examine quantitative findings. The qualitative data from the focus group was carefully reviewed multiple times for emergent themes while employing inductive and frequency analysis. Method triangulation was then performed as a means to gain further insight into the findings.

The findings revealed that the aquatic exercise intervention did not have global effects on cognitive function or physiological parameters. However, a statistically significant $(\mathrm{p}=0.014)$ change favoring the intervention group was found for the spatial working memory (SWM) between errors score. Qualitative and quantitative data converged to denote no global change to executive function while displaying improvements in cognitive parameters aligned with SWM. Statistically significant positive changes were observed in the DBP $(p=0.014)$ favoring the intervention group, however results from the 6-minute walk test as well as SBP and RHR only displayed positive trends without reaching statistical significance. Changes over time in body weight and composition between the treatment and control group were not statistically significant. Triangulation revealed compatibility of qualitative and quantitative trends for distance walked and global wellness improvements. A lack of commentary concerning changes in body weight and body composition reflected the negligent quantitative change in these parameters also denoting convergence of data. 
In relation to psychosocial mediators, no statistically significant interactions between the intervention and control groups over time were found. Initial survey results revealed very few perceived barriers, high motivation, sound social support, and high self-efficacy from both the intervention and control groups presenting a potential ceiling effect upon post-test findings. A statistically significant group effect in the control group was noted for social support showing a perceived reduction in social support. Qualitative data corroborated these findings for the intervention group with predominantly positive, voluminous responses in reference to all psychosocial mediators discussed. Barriers were accounted for yet traversed with solid coping strategies; motivation was high within multiple factors producing great motive for program commencement and continuation; self-efficacy was positively perpetuated throughout the course of the intervention via health outcomes and ability levels; and social support was strong through multiple cohort channels.

The exercise intervention was built around an adult education framework consisting of (1) finding motivation to begin exercise, (2) begin integrating exercise that fits into individual lifestyles, and (3) maintain and gain on all fitness parameters. It seems that through the information provided by the focus group participants that a behavior change did occur for the vast majority of the intervention group. With $86 \%$ of the attendees devising a plan for continuation of physical activity in conjunction with multiple variations in lifestyle changes and benefit recognition, it seems that the intervention group may be newly initiated chronic exercisers.

Qualitative findings denoting the intervention participants resolve to continue exercise may be due in part to their improved accessibility to exercise. Utilizing aquatic exercise as a gateway to provide knowledge, advance health and function, and improve self-efficacy while 
reducing barriers to exercise were all distinguishable reasons for using aquatic training as a precursor to other forms of exercise. Qualitative findings provide a more personal and complete picture of how aquatic exercise was able to offer the option of physical activity to those with the perception that they were not able to take part in exercise.

In conclusion, this study revealed that aquatic exercise does positively affect selective components of executive function, cardiovascular fitness, and wellness. Whereas psychosocial mediators did not show improvement, the intervention groups' maintenance of a positive association with these mediators following a 10-week aquatic exercise intervention is encouraging. Additionally, with the allowance of active participation in exercise via the aquatic medium, participants' accessibility to exercise was promoted while the perceived improvements in physical ability endorsed a behavior change towards improved overall physical activity levels.

Future research is needed to elaborate and further justify the use of aquatic exercise for improving physiological and cognitive parameters. Procedural alterations concerning frequency and duration of aquatic exercise sessions, inclusion of participants within the spectrum of cognitive impairment, the addition of a variety of cognitive function assessments, as well as comparing the effects of land versus water on all parameters should be explored. Additionally, the implication that SWM may experience greater alterations and advancements in novice exercisers and sedentary individuals should be examined. 


\section{Acknowledgements}

I wish to acknowledge the support of my dedicated committee members and the West Virginia University College of Education and Human Resources. Without the assistant and guidance of Jim Rye, Sam Zizzi, Guy Hornsby, Pat Obenauf, and Mia Erickson, I could have never completed such a daunting task. Their efforts helped me to find myself as a learner and researcher. Their willingness to selflessly give of their time and expertise was both heartwarming and humbling. I would like to give extra thanks to Jim Rye and Sam Zizzi. Without their guidance and gift of ample time and understanding towards my type-A needs I never would have made it through.

I would also like to give my gratitude to my loving husband, family, and friends to whom I would have never survived this process without. Though I stood resolute in my goal of bounding over every hurdle to get to the doctoral finish line, I was often reminded by my loved ones to remain focused (and that it was okay to be cranky...they would be there on the other side for me regardless of my continuously changing disposition). I could have asked for no better support system than what I am blessed with. 
TABLE OF CONTENTS

Table of Contents

LIST OF FIGURES

LIST OF TABLES

CHAPTER ONE: INTRODUCTION

Purpose

Problem and Significance

Theoretical Framework

Scope of Study

Research Questions

Basic Assumptions

Limitations

Definition of Terms

CHAPTER TWO: LITERATURE REVIEW

Executive Function

Age Related Change in Brain Structure

Cognitive Risk Factors

Normative Physiological Responses of Land Exercise Related to Executive Function

Effects of Exercise Modality on Executive Function

Exercise Environment

The Aquatic Environment

Exercise Implications for Memory Disorders

Theoretical Framework for Exercise and Cognitive Decline

Conclusion

Summary of Literature in Accordance with Research Question

Review of Purpose

Research Design

Participants

Treatments

Preliminary Testing

Intervention

Post Testing

Data Collection

Cambridge Neuropsychological Test Battery (CANTAB)

Health History

Demographic Data

Past year total physical activity questionnaire $\quad \frac{102}{102}$

Anthropometric Measurements

Resting Heart Rate

Blood Pressure

Psychosocial Mediator Surveys

6-Minute Walk Test

Focus Group

Adult Education

DATA ANALYSIS

Quantitative Data

Qualitative Data

Triangulation

$\underline{110}$

Research Question One

$\underline{110}$ 
Research Question Two $\quad \underline{111}$

Research Question Three $\quad \underline{111}$

Research Question Four $\quad \underline{112}$

Research Question Five $\quad \underline{112}$

Research Question Six $\quad \underline{112}$

CHAPTER FOUR: FINDINGS \& DISCUSSION $\quad \underline{113}$

Review of Purpose $\quad \underline{113}$

Review of Research Questions $\quad \underline{113}$

Participant Attrition and Attendance $\quad \underline{115}$

Review of Data Collection and Analysis $\quad \underline{116}$

Focus Groups $\quad \underline{117}$

Differences in Groups at Baseline $\quad \underline{118}$

Results

Research Question One $\quad \underline{120}$

Research Question Two $\quad \underline{132}$

Research Question Three $\quad \underline{139}$

Research Question Four $\quad \underline{143}$

Research Question Five $\quad \underline{155}$

Research Question Six

CHAPTER FIVE: CONCLUSIONS, IMPLICATIONS, \& RECOMMENDATIONS

Summary $\quad \underline{164}$

Review of Research Questions $\quad \underline{165}$

Methodology $\quad \underline{166}$

Findings $\quad \underline{169}$

Conclusions

Research Question One $\quad \underline{171}$

Research Question Two $\quad \underline{174}$

Research Question Three $\quad \underline{177}$

Research Question Four $\quad \underline{178}$

Research Question Five $\quad \underline{180}$

Research Question Six $\quad \underline{181}$

Implications for Practice and Policy $\quad \underline{182}$

Accessibility to Exercise $\quad 183$

Learning and Skill Acquisition $\quad 186$

$\begin{array}{ll}\text { Self-Imposed Ageism } & 188\end{array}$

$\begin{array}{lr}\text { Initiation versus Continuation } & 190\end{array}$

$\begin{array}{ll}\text { Physicians as Initiators } & 191\end{array}$

$\begin{array}{ll}\text { Exercise Maintenance } & 192\end{array}$

Spatial Working Memory and Novice Exercisers 193

Recommendations for Research $\quad \underline{195}$

$\begin{array}{ll}\text { Procedural Alterations } & 196\end{array}$

Advancing Aquatic Literature 199

Aquatic Exercise as a Gateway $\quad 200$

Retirement Intervention Program 201

Physician Education Directive 201

Alterations in Spatial Working Memory 202

Effects of Adult Education on Exercise Initiation $\quad 202$

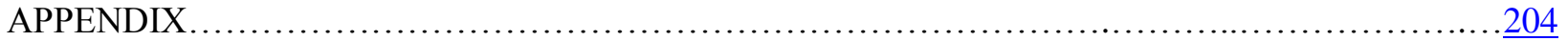

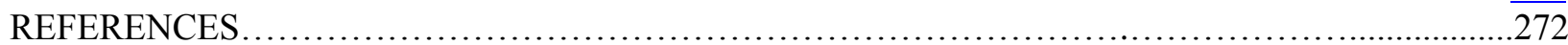




\section{List of Figures}

Figure $1.1 \quad$ Motivation Equation............................................... 11

Figure $3.1 \quad$ Research Design.............................................. 95

Figure 3.2 OTS Assessment................................................ 102

Figure $3.3 \quad$ PAL Assessment.................................................. 103

Figure $3.4 \quad$ SWM Assessment................................................ 104

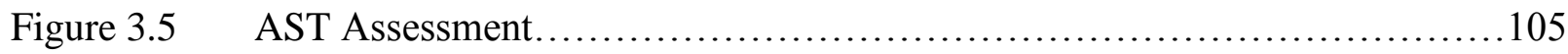

Figure $3.6 \quad$ RTI Assessment.................................................. 105

Figure 4.1 Pre-Post Spatial Working Memory Strategy Score..........................129

Figure 4.2 Pre-Post Spatial Working Memory Between Errors Scores...................130

Figure 4.3 Pre-Post Scores for Distance Walked in Meters..........................138

Figure 4.4 Pre-Post Scores for Diastolic Blood Pressure............................139

Figure 4.5 Percentage of time spent in Target Heart Rate Zone.......................140

Figure 4.6 Average Rating of Perceived Exertion.................................141

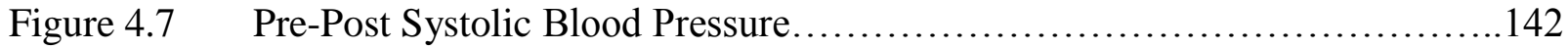

Figure 5.1 Sedentary Lifestyle in Comparison to Introduction of Aquatic Exercise.......188

Figure $5.2 \quad$ Continuation of Expansion for Exercise............................... 198 


\section{List of Tables}

Table $1.1 \quad$ Identified Barriers to Exercise in the Elderly............................... 10

Table 2.1 Major Risk Factors for CVD and their Association with Cognitive Decline or Alzheimer's Disease...............................................45

Table 2.2 Physiological and Performance Changes Associated with Aerobic Exercise and Cardiovascular Fitness ............................................60

Table 2.3 Contraction Variations Occurring in an Aquatic Environment.........................74

Table 4.1 Comparative Demographic Data for Control and Intervention Groups..........123

Table 4.2 Cognitive Function Descriptive Statistics for Pre-Test Intervention and Control Group Participants.................................................. 124

Table 4.3 Executive Function Battery Assessments, Parameters, and Outcome

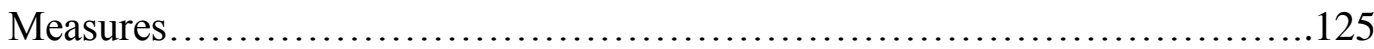

Table 4.4 Mean Values for One Touch Stockings of Cambridge Outcome Measure......127

Table 4.5 Mean Values for Paired Associates Learning Outcomes......................128

Table 4.6 Mean Values for Spatial Working Memory Outcomes........................131

Table 4.7 Mean Values for Attention Switching Task Outcomes......................132

Table $4.8 \quad$ Mean Values for Reaction Time......................................... 133

Table $4.9 \quad$ Mean Values for Cardiovascular Fitness Variables........................ 142

Table 4.10 Mean Values for Wellness Parameters of Body Weight \& Percent Body Fat....144

Table 4.11 Mean Values for Psychosocial Mediator Variables...........................148 


\section{Chapter One Introduction}

The aging process is comprised of many regular changes that occur in a maturing population across a lifespan. Both physical and cognitive decrements have been noted with increasing age resulting in accumulative concern for quality of life, medical costs and loss of independence. Connections to increasing age have been linked to cognitive decline after peaking during early adulthood (Hillman et al., 2009; Park \& Reuter-Lorenz, 2009) and symptoms have been noted to begin rapidly accelerating after age 60 (Barnes et al., 2007) with adults age 85 and older reported to have a rate of dementia of nearly 50\% (Park \& Reuter-Lorenz, 2009). This neurocognitive frailty is a serious concern for the aging population; it affects an individual's ability to age efficaciously and may be one of the largest threats to successful aging in our society (Park \& Reuter-Lorenz, 2009). Age related declines have been noted in a multitude of memory constituents including speed, short term memory, long term memory, and working memory. These decrements have been distinguished in both the 'normal aging' population (persons of advancing age without dementia) as well as in individuals with associated memory impairment, mild cognitive impairment (MCI), Alzheimer Disease (AD) and generalized dementia.

With the increasing concern for neurocognitive decline and escalating cognitive-related disease rates, research focused on determining causes, accelerants, impeding factors, and preventative strategies has become a focus of interest within the field. One of the principal points of study revolves around the role that exercise plays in the maintenance or fortification against neurocognitive decline. The preventative and protective effects of exercise on cognitive function have been well documented in the literature though no single theory has been adopted as to why this phenomenon occurs (Park \& Reuter-Lorenz, 2009). What is accepted, is that 
individuals that consistently participate in physical activity or were physically active during early-to-mid adulthood experience less cognitive decline and are at a reduced risk for developing AD and Parkinson's Disease (PD) later in life (Small, 2002; Radak et al., 2010). In fact, some researchers suggest that almost $50 \%$ of aging decline is preventable through positive lifestyle changes such as regular physical activity (O'Brien \& Vertinsky, 1991; Barnes \& Yaffe, 2011). There is also considerable evidence that exercise started even in old age has substantial cognitive, as well as physical benefit (Denkinger, Nikolaus, Denkinger, \& Lukas, 2012;

Middleton, Barnes, Lui, \& Yaffe, 2010; Barnes \& Yaffe, 2011). Moreover, physical inactivity and lack of participation in structured exercise have been identified as risk factors for cognitive decline and dementia-related diagnoses (Snowden et al., 2011; Radak et al., 2010; Chuang et al., 2013). Exercise has become such an important intervention strategy against cognitive decline that the American College of Sports Medicine (ACSM) concluded in an official 'Position Stand' that physical activity reduces cognitive decline associated with aging (Chodzko-Zajko, Proctor, Flatarone Singh, Minson, Nigg, Salem, \& Skinne, 2009).

A link between the positive effects of land-based cardiovascular exercise on executive function has been well established, though aquatic-based cardiovascular exercise has not been studied. The aquatic environment possesses many inherent characteristics that may increase the accessibility of exercise for the older adult. The environment may also potentially provide added stimulus to further enhance executive function via physiological mechanisms and environmental enrichment.

\section{Purpose}

The primary purpose of this study was to investigate the relation between aquatic-based cardiovascular exercise and executive function in older adults. This investigation was further 
reinforced by the inclusion of support questions concerning psychosocial mediators, exercise accessibility, adult education theory, physical activity level, and measurement of change in fitness and wellness parameters. This study aimed to link many areas including (a) the value and effectiveness of aquatic exercise in improving fitness and wellness parameters, (b) how aquatic exercise may diminish barriers to exercise - (c) with an emphasis on accessibility, (d) the impact of aquatic group exercise on social support, (e) self-efficacy, and (f) motivators. The final objective that was studied included the (g) relation and effectiveness of infusing adult education theory into exercise instructional methods.

\section{Problem and Significance}

The aging adult population has become a primary focus in the United States due to the increase in longevity and the rapidly escalating number of older Americans. Currently, older adults comprise approximately 13\% (39.6 million) of the population but that number is expected to grow to 19\% (72.1 million) by the year 2030 (Administration on Aging, 2011). With the expected boom in the elderly, the nation is looking towards impending issues and implications directly related to the older adult. One of the primary concerns is the impact that this population shift will have on our healthcare system, potentially placing unprecedented demands on agerelated and health care services. For this reason programs promoting healthy lifestyles and behaviors, clinical preventative services, education on planning for serious illness, mental health, and deterrence or impedance of cognitive impairment are being investigated, created, and promoted (CDC, 2011).

Participation in exercise and the inclusion of regular physical activity is noted to elicit positive results affecting many of the expressed concerns regarding advancing age. For this reason, exercise has become an important intervention strategy against the negative physical and 
cognitive outcomes commonly associated with ageing. It provides an opportunity to maintain and promote both physical and cognitive health within a single intervention strategy. The variety of exercise modalities is seemingly endless and, regardless of the type of exercise performed, the outcomes are consistent in positively impacting somatic and cerebral function. In fact, it is proposed that by participating in 180 minutes of activity per week, health care costs could be reduced by over $\$ 600$ for each active older adult each year (Shepard, 1987). Thus it seems as though the solution to the approaching age crisis has been found. The issue then, is how to get older adults to become more physically active.

Physical Activity and Public Health in Older Adults: Recommendation from the American College of Sports Medicine and the American Heart Association, a policy published by the Centers for Disease Control and Prevention (CDC), recommends 150 minutes of moderate intensity (3.0-6.0 METs) aerobic activity or 75 minutes of vigorous activity ( $\geq 6.0$ METs) per week (Nelson et al., 2007). It is estimated that only 9-26\% of older adults are meeting these recommendations in the U.S. (Tucker, Welk, \& Beyler, 2011) with over 50\% proclaiming no intention to begin an exercise program (Dishman \& Ickes, 1981). It is also evident that physical activity progressively decreases with increasing age (Schutzer \& Graves, 2004). The vast majority of older adults have a tendency to allocate the greater part of their time to sedentary behaviors (behaviors $\leq 1.5 \mathrm{METs}$ ) resulting in atrophy of the body and brain (Evenson, Buchner, \& Morland, 2012; Dogra \& Stathokostas, 2012). This staggering statistic leads to the question of why physical activity rates are so low within this population.

Barriers to Exercise in Older Adults. There are a variety of barriers for older adults associated with participation in physical activity. Health, environment, education, and exercise history are the most commonly cited reasons that older adults do not participate in exercise 
(Schutzer \& Graves, 2004). Physical impediments and limitations can highly affect activity tolerance and adherence while issues with strength, endurance, balance, or cardiovascular efficiency can have a direct impact on an older adult's choice and ability to participate in physical activity. Poor health, including pain and illness, are noted as the primary contributor to sedentary behavior in the elderly (Booth, Bauman, Owen, \& Gore, 1997; Cohen-Mansfield, 2003; Schutzer \& Graves, 2004). These physical factors are often augmented by a lack of knowledge on the benefits, appropriate modes and durations, proper execution, and availability of exercise (O'Neill \& Reid, 1991; Booth et al., 1997; Burton, Shapiro, \& German, 1999; Schutzer \& Graves, 2004). Collectively, these barriers make persuading the elderly to include and maintain physical activity within their daily lives a daunting task.

In 2001, the National Blueprint: Increasing Physical Activity Among Adults Age 50 and Older was released as an outline for a national strategy to increase physical activity among this population (Sheppard et al., 2003). Within this document, six major categories of barriers were identified as: research, home and community, workplace, medical systems, public policy and marketing and communication barriers (Park et al., 2010) (table 1.1). By identifying and defining these barriers, programming solutions may be more clearly remedied to allow for greater inclusion and accessibility to exercise in the aging population. 
Table 1.1: Identified Barriers to Exercise in the Elderly

\begin{tabular}{ll}
\hline Barrier & Definition \\
\hline Research Barriers & $\begin{array}{l}\text { Research barriers refer to the lack of guidance on duration and mode of } \\
\text { exercise necessary for positive health outcomes. }\end{array}$ \\
$\begin{array}{l}\text { Community or Home } \\
\text { Barriers }\end{array}$ & $\begin{array}{l}\text { Poor, unsafe or discouraging community design as well as a lacking } \\
\text { educational base for self-administered home-based programs are } \\
\text { denoted as another primary reason behind lack of physical activity in } \\
\text { older adults. }\end{array}$ \\
Workplace Barriers & $\begin{array}{l}\text { Employers may be a positive source for education and inclusion of } \\
\text { physical activity programs resulting in a cost-effective dissemination of } \\
\text { physical activity knowledge and practice. }\end{array}$ \\
Medical Systems & $\begin{array}{l}\text { Medical professionals receive minimal education relating to disease } \\
\text { prevention and thus are not fully capable of providing advice on or } \\
\text { referral for physical activity to their patients. }\end{array}$ \\
Public Policy Barriers & $\begin{array}{l}\text { A fragmentation of public policy organizations and lack of economic } \\
\text { models result in lack of financial support for the promotion of physical } \\
\text { activity. }\end{array}$ \\
Marketing and & $\begin{array}{l}\text { There is a lack of definition of the older population's perceptions, } \\
\text { beliefs, and concerns about physical activity and aging paired with } \\
\text { ineffective communications for education. }\end{array}$ \\
\hline A &
\end{tabular}

Adapted from L.W. (Poon, W.Chodzeko-Zajko, \& PD.Tomporowski, 2006)

Evident by the diversity of barriers associated with the inclusion of physical activity in the aging population, it may be prudent to approach interventions in a multilevel manner (Bauman et al., 2012; Sallis JF, Owen N, \& Fisher EB, 2008; SJH Biddle, W Brehm, M Verheijdn, \& M Hopman-Rock, 2012; SJH Biddle et al., 2012). Enhanced opportunity for success may be achieved by including information concerning individual, social, environmental, and policy components into the framework of physical activity program design (Bauman et al., 2012; Sallis JF et al., 2008). 
Motivators to Exercise in Older Adults. Motivation is the culmination of intrinsic and extrinsic factors that act on or within an individual to initiate or maintain behavior (Phillips, Schneider, \& Mercer, 2004). These factors are both individual and diverse among all populations, including the elderly. Motivating older adults to begin and adhere to regular physical activity is a challenging, yet critical element to program initiation and success (Phillips et al., 2004; SJH Biddle et al., 2012). Geelen and Soons propose that 4 subjective factors perceive the prognosis for success in physical activity participation and continuation: perceived chance of success (self-efficacy), perceived importance of the goal, perceived cost (physical or monetary), and inclination to remain sedentary (figure 1.1) (Geelen \& Soons, 1996). The appraisal of these 4 factors activate adoption or rejection of the behavior being contemplated and thus should be considered when developing and marketing exercise programming for any cohort, including those advancing in aging.

\section{Motivation $=$ Perceived Chance of Success X Perceived Importance of the Goal Perceived Cost X Inclination to Remain Sedentary}

Figure 1.1: Motivation Equation

There is an interesting relation between exercise barriers and exercise motivators in the aging population. Many identified barriers have also been recognized as motivators to participate in physical activity (Cohen-Mansfield, Marx, \& Guralnik, 2006). Issues including deteriorating health, increased time availability, being educated or referred by a physician to exercise, and closer proximity to exercise facilities play the role of both barrier and motivator in the elderly (Cohen-Mansfield et al., 2006; Schutzer \& Graves, 2004; Schutzer \& Graves, 2004). 
This dichotic relation illustrates the complexity of necessary intervention strategies in this population while providing potential for persuasion to participate in physical activity.

Self-Efficacy, Exercise and Older Adults. Self-efficacy, or the individual's belief that they will be able to perform a specified act or behavior, is consistently identified as playing a vital role in adherence to physical activity (Bauman et al., 2012). This principal is a central factor for Bandura's social cognitive theory proposing that environment, personal factors, and behavior interact to produce a level of belief in individual ability (Bandura, 2001; Bandura, 2004). In this construct, the idea of the individual's self-efficacy in relation to their perceived incentive must result in a positive, valued outcome or consequence (Bandura, 2004). Both shortterm and long-term outcomes are often sought as incentives for participation. In relation to physical activity, outcomes may include a feeling of accomplishment, enhanced energy levels, or improvements in health or physical status (cardiovascular endurance, muscular strength, muscular endurance, body composition, and flexibility). Though these benefits are likely to transpire with the addition of exercise, initial and continued participation hinges on an individual's self-efficacy relative to being able to complete the task (Bandura, 2004). Assurance that the individual's self-efficacy will promote participation in exercise could be assisted by including educational components (i.e. clear instructions), skill development, and a model of attainable behavior (Seefeldt, Malina, \& Clark, 2002).

Self-efficacy has been viewed as an important psychosocial mediator at any age, including within the elderly. Improvements in self-efficacy have been shown to increase participation and adherence in physical activity (Brassington, Atienza, Perczek, DiLorenzo, \& King, 2002) particularly when paired with achievable goals that are gradually advanced and encouraged (Marks, Allegrante, \& Lorig, 2005). Activities that are not easily achievable may 
not be appropriate for enhancing or maintaining self-efficacy related to exercise for the novice, or cognitively impaired exerciser. Finding a balance between providing achievable goals and ample challenge may afford the perfect paradigm for exercise programs targeting the aging population.

Social Support for the Exercising Elderly. Social support is comprised of many varieties of supplied support including: instrumental, informational, emotional, or appraising (Israel BA \& Schurman SJ, 1990; Zimet GD, Dahlem NW, Zimet SG, \& Farley GK, 1988). Within the realm of exercise, these components of social support may play the role of transporting an individual to an exercise class (instrumental), educating someone about the importance of exercise (informational), checking in on how an individual is doing in a newly started program (emotional), or providing feedback or reinforcement during skill allocation (appraising). Any one of these social support components may be provided by family, friends, exercise instructors, or physicians (SJH Biddle et al., 2012; Seefeldt et al., 2002). The level of importance for each of these constituents is going to vary individually, but social support as a whole has been consistently shown to be associated with advancements in activity levels (Bauman et al., 2012).

Social support in the aging population has been reported to increase exercise frequency, duration, and adherence (Walsh, Pressman, Cauley, \& Browner, 2001; Resnick, Orwig, Magaziner, \& Wynne, 2002; Rhodes, Martin, \& Taunton, 2001; Kassavou, Turner, \& French, 2013). Though social support may be an important psychosocial mediator when encouraging exercise success, the availability of social support seems to decrease with age (Resnick et al., 2002; McAuley, Jerome, Elavsky, Marquez, \& Ramsey, 2003). Creating outlets for social 
support within a group exercise environment may be a promising concept for integration of this psychosocial correlate of exercise.

Exercise, the Aquatic Environment, and Older Adults. There are a wide variety of exercise modalities that individuals can participate in to gain fitness benefits. These modalities can be divided into two primary categories: aerobic exercise and anaerobic exercise. Though both aerobic and anaerobic training can yield great benefits to the aging population pertaining to fitness and function, aerobic training is the predominate study focus for positive alterations in cognitive function. Aerobic training consists of activities utilizing large muscle groups to produce continuous, rhythmic movement that can be sustained over a longer period of time. It is dependent on aerobic metabolic systems that require large amounts of oxygen to produce the energy necessary for sustaining movement. These activities are usually of longer duration and performed at low to moderate intensity.

Aerobic training has been noted to have both acute and chronic effects on various components of executive function (Liu-Ambrose et al., 2010; Baker et al., 2010; Barnes, Yaffe, Satariano, \& Tager, 2003; Bielak, 2010; Budde, Voelcker-Rehage, Pietrabyk-Kendziorra, Ribeiro, \& Tidow, 2008; Davis et al., 2007; Davis et al., 2007; Davis, Dennis, Daselaar, Fleck, \& Cabeza, 2008; Liu-Ambrose et al., 2008; Swardfager et al., 2010; Teixeira et al., 2012; Hogan, Mata, \& Carstensen, 2013; Joyce, Smyth, Donnelly, \& Davranche, 2014). Research supports the link between participation in chronic aerobic exercise and: improvements in spatial learning, memory, cognitive capacity, selective and divided attention, working memory, cognitive flexibility, planning, inhibition, decision making, problem solving, cognitive speed, and overall cognitive function (Angevaren, Aufdemkampe, Verhaar, Aleman, \& Vanhees, 2008; Baker et al., 2010; Barnes et al., 2003; Birren \& Fisher, 1995; Budde et al., 2008; Fabre, Chamari, Mucci, 
Masse-Biron, \& Prefaut, 2002; Hillman et al., 2009; Hillman, Erickson, \& Kramer, 2008; Hillman, Snook, \& Jerome, 2003; Ke, Huang, Liang, \& Hsieh-Li, 2011; Liu-Ambrose et al., 2010; Man, Tsang, \& Hui-Chan, 2010; Scherder, Eggermont, Geuze, Vis, \& Verkerke, 2010; Smith et al., 2010; Van der Borght, Havekes, Bos, Eggen, \& Van der Zee, 2007; Teixeira et al., 2012; Yaguez, Shaw, Morris, \& Matthews, 2011). The acute and chronic effects of aerobic training are widely documented and provide some insight linking the effects of exercise with executive function. The overall outcome of exercise is enhanced immune condition of the brain and its structures that results in improved neural plasticity thus promoting and preserving cognitive function.

The introduction of the aquatic environment as the medium in which to perform aerobic exercise training may provide a multifaceted reasoning for its implementation. The environment that an individual is immersed in can play a major role in the maintenance and development of executive function (Bielak, 2010; Zec, 1995). An environment that provides the inhabitants a means of stimuli, known as an 'enriched environment', affords the optimal condition for cognitive function. This stimulus could be obtained from exercise, cognitively challenging activities, or a complex environment. The aquatic environment affords various new stimuli to further develop the exercise session from mere exercise to exercise within an enriched environment. The challenges that the physical attributes of the aquatic environment lend to individuals in the pool also drive the requirement for added concentration, variance of muscular contractions and coordination while performing aquatic exercise. The buoyant support that the water provides may increase comfort during exercise for a variety of populations allowing them to enjoy the exercise experience more while promoting participation longevity. Lastly, the increase in circulatory function that augments blood availability may potentially lead to 
increased cerebral circulation which has been definitively seen to promote neural structure maintenance and growth (Barnes et al., 2003; Bielak, 2010; Deeny et al., 2008; Lachman ME, 2006; Man et al., 2010; Scherder et al., 2010; Small et al., 2006).

Exercise Accessibility and Elderly. As previously discussed, physical barriers to participating in exercise are one of the most noted reasons elderly individuals do not exercise (Booth et al., 1997; Cohen-Mansfield, 2003; Schutzer \& Graves, 2004). Exercising in the water can provide the aging population with a valuable alternative to seemingly inaccessible land exercise. The physical properties of water can be utilized and manipulated to offer support, assistance, and resistance depending on the needs of the individual. The aquatic environment can provide an atmosphere that reduces risk of injury and pain, delivers physiological support for those with less than optimal health status, and, with the inclusion of a group fitness class with a qualified instructor, can provide social support and exercise education (Becker BE \& AJ Cole, 2011; Belza, Topolski, Kinne, Patrick, \& Ramsey, 2002).

Exercise and Adult Education. According to the literature, there is a necessity for exercise interventions to take a multifaceted approach when working with the ageing adult (Bauman et al., 2012; Sallis JF et al., 2008; SJH Biddle et al., 2012; SJH Biddle et al., 2012). The inclusion of an educational component has become an important constituent within the integrated approach to physical activity programming for the aging adult (Schutzer \& Graves, 2004; O'Neill \& Reid, 1991). It serves the role of enlightening individuals on the importance, benefits, methods, and by what means to participate in physical activity. Though education about exercise has been, and should be, used as a tool to promote the inclusion of physical activity into the lifestyles of any population, it seems to be of particular importance within those of advancing age (Schutzer \& Graves, 2004; O'Neill \& Reid, 1991). 
Adult educational strategies are very different from approaches utilized to teach children. This is an important fact to recognize in order to have the greatest effect on the individuals being targeted to address lifestyle changes. In order to gain the attention and interest of those that need to be educated on the importance of physical activity, a positive learning climate must be developed (Sullivan R.L., Arnold, \& Sarkees, 1990). This is true of any educational moment that may present itself to motivate individuals to begin exercise as well as the continued education that should be provided during exercise. Establishing a positive environment for individuals to feel comfortable learning, making mistakes, and asking questions throughout the acclimation process of exercise could be imperative to their continuation of physical activity as a whole. The process of providing education on the 'why' of exercise may also extend the individuals inclinations to exercise if the reasoning aligns with the adults needs and wants (Lorge, 1947). Providing opportunities for the aging adult to select their own experience via providing options for physical activity integration may also be assistive in the acclimation and learning process. Many adult learning theorist believe that adult learning is rooted in a wealth of past experiences, self-direction, personal needs and interests, usefulness of the information or skill, and the amount of onus they can link to the experience (Knowles M.S., Holton III, \& Swanson RA, 2011). If these components can be built-in to the exercise experience, there may be a greater effort and adherence response leading to superior outcomes.

\section{Theoretical Framework}

Physical Activity as a Learned Behavior. Taking part in physical activity is a behavior that is considered an important component in promoting and maintaining a healthy physical and mental status. Despite the known benefits, it is estimated that less than a quarter of older adults are currently meeting recommended physical activity recommendations (Tucker et al., 2011) 
with over 50\% proclaiming no intention to begin an exercise program (Dishman \& Ickes, 1981). Though clear recommendations have been outlined by ACSM for physical activity in the aging population, little research within theoretical frameworks has been conducted to guide practitioners on exercise integration (Nelson et al., 2007).

Theories and models of human behavior can assist in the development and refinement of physical activity intervention strategies. Theories including factors that influence adult physical activity such as: social support, self-efficacy, barriers, and activity enjoyment while infusing adult learning theories may be efficacious for incorporation or maintenance of a new behavior such as exercise. There are three evident thresholds to clear prior to being able to achieve a daily physical activity regimen: (1) Finding motivation to begin exercise, (2) Begin integrating exercise that fits into individual lifestyles, and (3) Maintain and gain on all fitness parameters. These three steps have many complex theoretical components that underlie the success and magnitude of the intervention attainment.

Finding Motivation to Begin Exercise in Older Adults. Exercise barriers and motivators in the aging population have been shown to mirror one another (Cohen-Mansfield et al., 2006). Issues including deteriorating health, increased time availability, being educated or referred by a physician to exercise, basic exercise knowledge, and proximity to exercise facilities play the role of both barrier and motivator in the elderly (Cohen-Mansfield et al., 2006; Schutzer \& Graves, 2004; Schutzer \& Graves, 2004). These motivating barriers may provide a gateway to stimulate beginning an exercise program and can be tied to theoretical models including: Health Belief Model, Social Cognitive Theory, and Social Support. Utilizing components from each of these theories and models to shape intervention strategies focused on the aging adult could provide powerful incentive to begin physical activity. 
Poor health is among the most frequently cited barriers and motivators in older adults (Schutzer \& Graves, 2004; Cohen-Mansfield et al., 2006; Booth, Owen, Bauman, Clavisi, \& Leslie, 2000). Health related behavior, such as participation in physical activity, is noted to be dependent on an individual's perception of: severity and susceptibility of illness, benefits of preventative action, and barriers to that action (Rosenstock, Strecher, \& Becker, 1988;

Rosenstock, 1988). Many older adults do not associate participation in regular exercise with improvements in physical health or believe that they achieve enough physical activity via their activities of daily living (ADLs)(O'Neill \& Reid, 1991; Schutzer \& Graves, 2004). Due to the level of importance that older adults tie to health status, this could provide an important starting point for education and guided motivation in beginning an exercise program. Utilizing adult learning theory to provide educational resources to highlight the benefits of physical activity on health and wellness could be paramount for encouraging the adoption of physical activity practices in older adults.

The inclusion of self-efficacy, or the individual's belief in their ability to complete a task, is noted to be a strong and consistent factor in beginning physical activity (Schutzer \& Graves, 2004). Self-efficacy plays an integral role in Bandura's social cognitive theory and has also been integrated into the health belief model (Bandura, 2004; Rosenstock, 1990; Bandura, 2001). When concerning the adoption of physical activity, performing exercise must outweigh the negative expectations and associations the individual links to performing exercise. In addition, there must be value tied to the short and long term consequences and outcomes associated with physical activity. Improving self-efficacy for physical activity may be encouraged by providing opportunities for development of the skill-set, designing or explaining the behavior so that it seems attainable, or modeling the desired behavior (Collins JL, 1995). 
Lastly, social support for physical activity can play a variety of roles when motivating an older adult to begin exercising. Instrumental, emotional, appraising, or informational social support can all assist in providing the ageing adult with inspiration or implement to become active (Israel BA \& Schurman SJ, 1990). Social support for physical activity may range from providing transportation, informing someone about an exercise opportunity, or eliciting care or concern and may be provided by family, friends, fellow exercisers, or exercise instructors.

By bridging all of these theoretical components together, interventions may be able to provide the older adult with a plethora of reasons to begin exercising. While identifying any impending barriers to success and improving self-efficacy, this cohort will be educated about the benefits and opportunities of initial exercise participation.

Integrating an Exercise Program for Older Adults. It has been suggested that modifications in behavior are more attainable when broken down into smaller, more achievable segments when the behavior is complex in nature (Michael, 2003; Skinner BF, 1953). Thus, if 150 minutes of moderate intensity exercise is recommended for older adults, the behavior could be broken down and introduced by first requesting that the individual walk for 10 minutes daily. Once that behavior has been accepted, additional time could be added until the goal is met. The simplicity of this behavioral plan is complicated by competing behaviors that may be satisfying, habitual, or prompted by the environment (Michael, 2003; Skinner BF, 1953). Due to these multifaceted external stimuli, the importance of reinforcement and anticipated rewards as encouragement towards maintaining the newly adopted behavior may play an important role.

Encouragement and reinforcement may come in the way of social support from the exercise leader or fellow exercise class participants. Social support may play a role in the onset of exercise but it is also very important when time allocation or difficulty level for exercise 
increases. The variety of types of social support that can promote taking part in a physical activity program and continuing on with that program could provide participants continued motivation and enjoyment.

Equally important is the further development of self-efficacy related to physical activity. Self-efficacy is believed to be the single most important behavior determinant for beginning an exercise program and its level of significance stands for continuation of that behavior (Bandura, 2004). Once an exercise program has been initiated, the focus for enhancing and improving selfefficacy may shift to instructional clarity, presenting an attainable exercise program, skill development, and creating an exercise environment that evokes trust and respect (Bandura, 2001). By building an environment that individuals feel safe to learn exercise techniques and build on existing skills, self-efficacy can be fostered.

Maintain and Gain on Fitness Parameters for Older Adults. Fitness parameters important for maintenance and improvement of quality of life, overall function, and wellness can begin with the foundational components of fitness: cardiovascular endurance, muscular endurance, muscular strength, flexibility, and body composition. Once this foundation is created, performance related components can be incorporated. Though titled performance related fitness parameters; power, speed and quickness, agility, balance, and motor skill development are necessary for functional improvements when assessing ADLs.

Many activities necessary to accomplish in order to establish and build the performance related fitness components may be challenging for novice exercisers, in particular, elderly novice exercisers. Because of this level of difficulty, imposing methods that employ adult learning theory while continuing to foster self-efficacy and social support amongst the group and instructor will be paramount for continued participation. It may also be of great benefit to 
perform activities that can illustrate positive change that has been acquired throughout the exercise sessions. Flexibility, endurance, strength, balance, and many other components of fitness can easily be assessed prior to beginning the program and reassessed throughout the continuation of the program to show improvements to provide continued positive reinforcement.

\section{Scope of the Study}

The study employed a convenience sample $(\mathrm{N}=44)$ of adult participants between the ages of 60-90 years old with no neurological defect (e.g., possible dementia, self-report of neurological disease such as multiple sclerosis, brain tumor, and Parkinson's disease). Participants were not currently involved in regular exercise training (e.g., running, cycling, swimming, weightlifting, etc.) defined as greater than 30 minutes of such activities more than 5 times per week (150 minutes in total). Participants were randomly assigned to the treatment or control group. Both groups of eligible participants received initial cognitive function testing and a field-based fitness test to determine baseline cognitive and fitness parameters. Demographic data, psychosocial mediator surveys, resting heart rate, resting blood pressure, a health history, and a physical activity questionnaire were also administered and collected. The participants in the treatment group then took part in a 10-week aquatic exercise intervention, during which they met for an expert-instructed, aquatic group fitness class at the WVU Natatorium 3 times per week lasting 60 minutes per session. The intensity of the exercise sessions were based on individual target heart rates and were progressively increase throughout the duration of the 10week time period. The control group was then offered this same 10-week aquatic exercise program after the study was completed.

Baseline physiological measures were established for current levels of cognitive function parameters, cardiovascular fitness, weight, hip-to-waist-ratio, body composition, resting heart 
rate, and resting blood pressure. In addition, baselines were measured for exercise barriers, social support, self-efficacy, and motivators. At the mid-point of the aquatic exercise intervention, the treatment group had their resting blood pressure and heart rate measured and psychosocial mediator surveys re-administered. All variable measures were then repeated at the conclusion of the 10-week program for both the treatment and control groups. These repeated measures were compared to baseline levels, so as to determine any effect the prescribed physical activity program may have had on all impending cognitive function, fitness and wellness, and psychosocial mediator parameters. A post-treatment qualitative focus group with members of the intervention group was completed in order to provide insight into the participants' attitudes, barriers or relations of exercise to ADLs and cognitive function.

Pilot Data. A pilot study was conducted to assist in informing the researcher's decisions concerning assessment selection and exercise administration for the proposed study. Institutional review board (IRB) approval was sought and received prior to beginning the study (H-24479). Five participants between the ages of 60-90 years old with no neurological defect were recruited to take part in this pilot study. All participants read and signed an informed consent (Appendix A) and obtained a medical release provided by their personal physician stating that they were cleared to participate in exercise. The study consisted of two preliminary visits followed by a 6week shallow water aquatic exercise intervention. Following the exercise intervention, participants were asked to take part in a final visit to reassess fitness and cognitive parameters.

This pilot study provided the researcher with valuable information to guide the proposed study. Modifications in assessments for cardiovascular fitness and cognitive function were reconsidered due to time allocation, participant ability, sensitivity of the measurement tool, and degree of change. The initial study utilized a submaximal $\mathrm{VO}_{2 \max }$ test performed on a treadmill 
or recumbent bicycle. Though this test is recognized as a preferred method for measuring cardiorespiratory fitness, it is very time consuming and three out of the five participants had difficulty walking on the treadmill due to lack of familiarization. The inability to properly walk on the treadmill comfortably can skew the results of this assessment. In addition to the lack of skill for treadmill walking, a mask was affixed to the participants to provide breath-by-breath analysis. Many of the participants stated their level of discomfort with the mask. Due to these factors, the researcher chose to seek another suitable assessment for cardiovascular endurance that would be appropriate for the aging population. The 6-Minute Walk Test was chosen to take the place of the treadmill test.

The cognitive screening tool that was highlighted in a wide array of studies was the Mini Mental State Evaluation (MMSE). This simply administered test did not provide enough sensitivity to change in the cognitively in-tact older adult. Due to this finding, the researcher explored other options noted in the literature and discovered CANTAB to be a much more suitable and sensitive tool to assess multiple parameters of cognitive and executive function.

Findings from this pilot study were used in the development of the currently proposed study. It promoted further investigation into more appropriate assessment tools for the targeted population which will assist in strengthening the outcomes and success of the proposed study.

\section{Research Questions.}

Research Question One. Research Question One asks: Does participation in a 10-week aquatic exercise class result in a statistically significant $(\mathrm{p} \leq .05)$ improvement in cognitive function when compared to the control group? The null hypothesis for Research Question One $\left(\mathrm{H}_{01}\right)$ states: Participation in a 10 -week aquatic exercise class does not result in a statistically significant $(\mathrm{p} \geq .05)$ improvement in cognitive function when compared to the control group. 
The researcher utilized the CANTAB® system for measuring several components of cognitive function including: working memory capacity, spatial working memory, orientation, registration, attention, calculation, language and recall. The system saved and stored all results from tests elicited which were exported into spreadsheets and analyzed using SPSS. Analysis was completed on each component of the assessed parameters.

Research Question Two. Research Question Two asks: Does participation in a 10-week aquatic exercise class result in a statistically significant $(\mathrm{p} \leq .05)$ improvement in cardiovascular fitness when utilizing the Kruel target heart rate deduction to configure individualized target heart rate zones when compared to the control group? The null hypothesis for Research Question Two $\left(\mathrm{H}_{02}\right)$ states: Participation in a 10-week aquatic exercise class does not result in a statistically significant $(\mathrm{p} \leq .05)$ improvement in cardiovascular fitness when utilizing the Kruel target heart rate deduction to configure individualized target heart rate zones when compared to the control group. Data from the 6-Minute Walk Test was the primary method used to monitor changes in cardiovascular fitness. Total distance, blood pressure readings, and heart rate responses taken pre-intervention and post-intervention will be compared to the control group utilizing a two-way ANOVA.

Research Question Three. Research Question Three asks: Do statistically significant $(\mathrm{p} \leq .05)$ positive changes occur for general fitness and wellness parameters after participation in a 10-week aquatic exercise class when compared to the control group? The null hypothesis for Research Question Three $\left(\mathrm{H}_{03}\right)$ states: No statistically significant positive changes $(\mathrm{p} \leq .05)$ occur for general fitness and wellness parameters after participation in a 10-week aquatic exercise class when compared to the control group. General fitness and wellness parameters 
were tested pre and post intervention via anthropometric measurements (weight and body composition), distance walked, resting blood pressure, and resting heart rate.

Research Question Four. Research Question Four asks: Does participation in a 10week aquatic exercise class result in a statistically significant $(\mathrm{p} \leq .05)$ alteration of social support, self-efficacy, motivators, or exercise barriers when compared to the control group? The null hypothesis for Research Question Four $\left(\mathrm{H}_{04}\right)$ states: Participation in a 10-week aquatic exercise class does not result in a statistically significant $(\mathrm{p} \leq .05)$ alteration of social support, self-efficacy, motivators, or exercise barriers when compared to the control group. Data collection for Research Question Four consisted of 3 participant surveys administered pre, mid, and post intervention. Surveys asked participants about barriers, motivators, self-efficacy and social support related to exercise. Responses from each of the surveys were appraised. Focus group transcripts and notes were also being reviewed for immerging themes concerning psychosocial mediators to further enrich the findings for Research Question Four.

Research Question Five. Research Question Five asks: Does the infusion of adult education theory into a 10-week aquatic exercise class assist in behavior change? Focus group transcripts and notes were reviewed for immerging themes and were analyzed qualitatively using the methods described by Patton (2002) concerning adult education theory and behavior change.

Research Question Six. Research Question Six asks: Does aquatic exercise provide participants with greater accessibility to exercise participation? Focus group transcripts and notes were reviewed for immerging themes and were analyzed qualitatively using the methods described by Patton (2002) concerning exercise accessibility. 


\section{Basic Assumptions.}

1. The Cambridge Neuropsychological Test Battery is a reliable and valid cognitive testing tool that is based on a normative data base, sensitive to small changes in various cognitive domains, and is culturally independent (Fowler, Saling, Conway, Semple, \& Louis, 2002; Yaguez et al., 2011; Egerhazi, Berecz, Bartok, \& Degrell, 2007; Cruise et al., 2011). The Past Year Total Physical Activity Questionnaire interview is a reliable and valid tool that measures all parameters of physical activity (frequency, duration, and intensity) within the previous 12 months (Friedenreich et al., 2006). The psychosocial mediator surveys are reliable and valid tools to assess exercise barriers, social support, self-efficacy and motivators (Marcus, Rossi, Selby, Niaura, \& Abrams, 1992; Zizzi, 2012; Resnick et al., 2002; McAuley et al., 2003; Marcus, Selby, Niaura, \& Rossi, 1992). The 6-Minute Walk Test assesses cardiorespiratory and functional fitness and can predict aerobic fitness in older adults (Enright, 2003; Rikli R.E. \& Jones C.J., 1998; Kervio, Carre, \& Ville, 2003).

2. The majority of the participants will adhere to and improve fitness across the 10 -week treatment intervention.

3. A 10 -week program consisting of 150 minutes of aquatic activity is sufficient time to observe improvements in fitness parameters (Heyn, Johnson, \& Kramer, 2008; Farrell, 2011; Heyn, Abreu, \& Ottenbacher, 2004; 2010).

4. Participants will accurately assess and report their perceptions of, as well as their responses to, the dependent variable measures. 


\section{Limitations of the Study.}

The generalizability of the conclusions derived from the analysis is limited due to the specific population recruited for this investigation. The sample was one of convenience based on individuals volunteering to participate; consequently participants in this study were predominately female so translation across genders is questionable. Thus, suggestions and implications for program recommendations may be restricted to female participants. Another limitation is the small sample size and relative statistical power. There were variables that neared reaching statically significant levels, however due to the small sample size and associated power; levels of statistical significance were more challenging to reach. In addition to these noted parameters, preliminary and post assessment times were made to meet the needs of the participant's schedules thus they did not take part during the same time of day. This could have had an impact on some of the physiological measures reliability and validity. Of note, a technical error was experienced during some of the final assessment period resulting in response slowing of the CANTAB ${ }^{\circledR}$ program during RTI. This error was addressed but not able to be fully remedied for the post-testing sessions. Lastly, focus group transcripts may have reflected social desirability due to the relationship formed amongst group members with the researcher. The researcher attempted to distance herself from the focus group by commissioning an unbiased mediator and recorder to reduce the threats of data collection bias.

\section{Definition of Terms.}

Accessibility to Exercise, refers to the degree to which exercise, including devices and the environment utilized, is available to as many individuals as possible. It may otherwise be defined as the ability to access and benefit from exercise (Rimmer, Riley, Wang, \& Rauworth, 2005). 
Activities of Daily Living, or ADL, is an activity necessary for daily care and independent living (Wiener, Hanley, Clark, \& Van Nostrand, 1990).

Executive Function, otherwise known as cognitive control or cognitive function, is a series of self-regulatory mechanisms that are utilized to process perception, memory and action (Hillman et al., 2008). It includes planning, initiation, sequencing, and monitoring of actions involving attention, concentration, abstraction, selectivity, mental flexibility, self-control and working memory (Hillman et al., 2008; Teixeira et al., 2012).

Focus Group Interview is an interview conducted with a small group of people focused on a specific topic (Patton, 1999; Patton, 2002).

Psychosocial Mediator, is a "intervening causal variable that is necessary to complete a cause-effect pathway between an intervention and a physical activity" (Bauman, Sallis, Dzewaltowski, \& Owen, 2002).

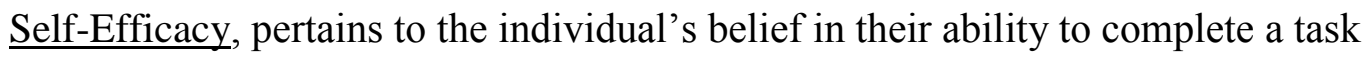
(Bandura, 2004).

\section{Acronyms.}

$\underline{\text { AST }}$ - Attention Switching Task

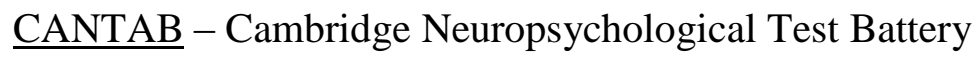

$\underline{\text { DBP }}$ - Diastolic Blood Pressure

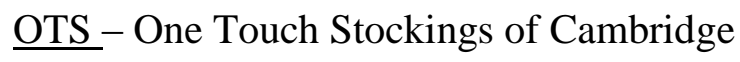

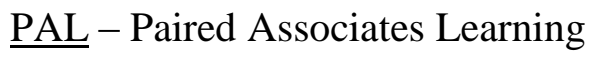

$\underline{\text { RHR }}$ - Resting Heart Rate

$\underline{\mathrm{RPE}}-$ Rate of Perceived Exertion

$\underline{\text { RTI }}$ - Reaction Time 
$\underline{\text { SBP }}$ - Systolic Blood Pressure

$\underline{\text { SWM }}$ - Spatial Working Memory 


\section{Chapter Two Review of Literature}

The aging process is comprised of many regular changes that occur in a maturing population across a lifespan. Both physical and cognitive decrements have been noted with increasing age resulting in accumulative concern for quality of life, medical costs and loss of independence. Connections to increasing age have been linked to cognitive decline after peaking during early adulthood (Hillman et al., 2009; Park \& Reuter-Lorenz, 2009) and symptoms have been noted to begin rapidly accelerating after age 60 (Barnes et al., 2007). Adults age 85 and older are reported to have a rate of dementia of nearly $50 \%$ (Park \& Reuter-Lorenz, 2009). This neurocognitive frailty is a serious concern for the aging population; it affects an individual's ability to age efficaciously and may be one of the largest threats to successful aging in our society (Park \& Reuter-Lorenz, 2009). Age related declines have been noted in a multitude of memory constituents including speed, short term memory, long term memory, and working memory. These decrements have been distinguished in both the 'normal aging' population (persons of advancing age without dementia) as well as individuals with associated memory impairment, mild cognitive impairment (MCI), Alzheimer Disease (AD) and generalized dementia. With the increasing concern for neurocognitive decline and escalating cognitiverelated disease rates, research focused on determining causes, accelerants, impeding factors, and preventative strategies has become a focus of interest within the field. One of the principal points of study revolves around the role that exercise plays in the maintenance or fortification against neurocognitive decline. The preventative and protective effects of exercise on cognitive function have been well documented in the literature though no single theory has been adopted as to why this phenomenon occurs (Park \& Reuter-Lorenz, 2009). What is accepted as fact is that individuals that consistently participate in physical activity or were physically active during 
early-to-mid adulthood experience less cognitive decline and are at a reduced risk for developing AD and Parkinson's Disease (PD) later in life (Small, 2002; Radak et al., 2010). There is also considerable evidence that exercise started even in old age has substantial cognitive, as well as physical benefit (Denkinger et al., 2012; Middleton et al., 2010). Moreover, physical inactivity and lack of participation in structured exercise have been identified as risk factors for cognitive decline and dementia-related diagnoses (Snowden et al., 2011; Radak et al., 2010; Barnes et al., 2013; Chuang et al., 2013). Exercise has become such an important intervention strategy against cognitive decline that the American College of Sports Medicine (ACSM) concluded in an official 'Position Stand' that physical activity reduces cognitive decline associated with aging (Chodzko-Zajko, Proctor, Flatarone Singh, Minson, Nigg, Salem, \& Skinne, 2009)

A link between the positive effects of land-based cardiovascular exercise on executive function has been well established, though aquatic-based cardiovascular exercise has not been studied. The aquatic environment may provide added stimulus to further enhance executive function via physiological mechanisms and environmental enrichment. The main purpose of the current study is to investigate the relation between aquatic-based cardiovascular exercise and executive function in older adults.

The causes of executive function decline in the aging population are multifactorial. Physiological, socio-contextual/environmental, and pathological circumstances or variables can all be contributing factors to the speed in which cognitive decline and decrements in executive function occurs within an individual. It is imperative to understand each component's influence on the decrements associated with cognitive dysfunction and the potential coping strategies utilized to impede the progression of the impairment. For this reason, a review of the structural changes in the brain associated with aging, risk factor identification and contributory influences, 
along with the normative effects of exercise on land and in water will be discussed in relation to executive function impairment.

\section{Executive Function}

Executive functioning, otherwise known as cognitive control, is a series of self-regulatory mechanisms that are utilized to processes perception, memory and action (Hillman et al., 2008). It includes planning, initiation, sequencing, and monitoring of actions involving attention, concentration, abstraction, selectivity, mental flexibility, self-control and working memory (Hillman et al., 2008; Teixeira et al., 2012). The primary utility of executive function is to support meaningful organization and control of complex, goal-oriented tasks (Buckner, 2004). Both long-term and working memory is governed by executive function due to the requirement of strategic processing and task performance (Buckner, 2004).

Working memory, a subcomponent of executive function, is the culmination of set shifting, updating, and inhibition (Coles \& Tomporowski, 2008; Liu-Ambrose et al., 2008). This includes manipulating temporarily stored new information in order to utilize it for current circumstances (Zec, 1995). Memory, in general, is thought to be a linear process that flows through a series of components or stages: short term (primary memory), working memory (manipulation of short term memory), long term memory (secondary memory), and tertiary memory (remote memory) (Zec, 1995). If slowing occurs at any point of the linear progression, memory function becomes impaired (Zec, 1995). Memory and attention are interdependent elements thus it is imperative to look at both variables (Teixeira et al., 2012). Attention denotes selection of a focus and allocating processing resources to that focus while inhibiting distracting stimuli (Chun \& Turk-Browne, 2007). Memory provides a limited capacity of what is to be stored, while attention determines the level of importance that the content being reviewed serves 
(Teixeira et al., 2012; Chun \& Turk-Browne, 2007). In other words, if the content attracts attention, it is more likely to be encoded into the memory. Memory also assists in attention via perception (Chun \& Turk-Browne, 2007). An individual must be able to match perceptual input with the exemplifications located in their stored memory (Chun \& Turk-Browne, 2007).

\section{Age Related Changes in Brain Structure}

Structural decline within the frontal, parietal and temporal lobes of the brain resulting in decrements in cognitive processes begin after early adulthood (after age 30) (Raz \& Rodrigue, 2006; Park \& Reuter-Lorenz, 2009; Leal \& Yassa, 2013). Total brain volume is reduced by approximately $5 \%$ per decade after age 40 with the greatest size reduction being observed in the caudate, cerebellum, hippocampus and prefrontal areas (Park \& Reuter-Lorenz, 2009; Scahill et al., 2003). There has also been evidence for declining volume of grey and white matter in the older adult with particular decrements being noted in the frontal and parietal cortex occurring after the fifth decade of life (Park \& Reuter-Lorenz, 2009; Raz \& Rodrigue, 2006; Voss et al., 2013; Jacobs et al., 2013). Dopaminergic receptors and synaptic density have similarly been shown to be reduced in quantity with age (Park \& Reuter-Lorenz, 2009) while neuronal shrinkage and dysmorphology, dendritic spine loss and neuronal body loss have similarly been noted with the aging process (Myers, 2008). Due to these structural alterations, information processing becomes less efficient. Processing speed, working memory capacity, and attention can all be adversely affected (Park \& Reuter-Lorenz, 2009). Other components of memory remain virtually untouched by the aging process, including: long term memory and implicit memory (Park \& Reuter-Lorenz, 2009).

A review of age related changes that occur in brain structure is necessary in order to fully comprehend the magnitude of the problem being investigated. It is imperative to assess all of the 
components associated with cognitive dysfunction in order to support theories behind treatment success.

Grey and White Matter. Grey and white matter are the two primary components of the brain. Grey matter, or substantiagrisea, is comprised of neuronal cell bodies, neutrophil, glial cells and capillaries (Guyton AC, 2011; Guyton AC, 2011). It is primarily controlled by the nerve cell bodies and dendrites allowing for the regulation of bodily senses, memory and muscle movement. White matter, also known as substantiaalba, is primarily composed of glial cells and myelinated axons that permit transmission of signals from the grey matter to other cerebral and somatic regions(Guyton AC, 2011). It dynamically affects learning via relaying and coordinating communication between regions of the brain through action potentials.

Decline in grey and white matter begins between the fourth and fifth decade of life and has been reported to result in attention and memory deficits including slowing of information processing resulting in a less efficient information transmission system (Birren \& Fisher, 1995; Myers, 2008; Peters, 2006; Jacobs et al., 2013). Resnick et.al. (2003) reported notable deterioration in grey and white matter in a period as brief as two years in very healthy older adults over the age of 59. Greater decline in volume and density in addition to structural damage in grey and white matter was greatest in the anterior or frontal regions revealing that there may be structures of the brain that are more susceptible to the effects of aging (Peters, 2006; Smith et al., 2010; Park \& Reuter-Lorenz, 2009). Reductions in grey matter have been reported to stem from neuronal cell death (Raz N, 2004; Anderton, 2002; Kolb, I, 1990; Jacobs et al., 2013) while white matter expresses deterioration of the myelin sheath (demyelination) (Bartzokis et al., 2003; Head et al., 2004). 
Studies utilizing exercise as a dynamic variable to investigate brain health and function have demonstrated improved executive function, attention, generalized cognitive function as well as reductions in the decline or even improvement in grey and white matter density (Barnes et al., 2003; Budde et al., 2008; Davis et al., 2008; Lautenschlager et al., 2008; Swardfager et al., 2010; Zlomanczuk et al., 2006; Voss et al., 2013; Jacobs et al., 2013). Exercise seems to selectively enhance areas that are most vulnerable to age related decline (Nichol, Parachikova, \& Cotman, 2007; Baker et al., 2010; Klusmann et al., 2010). Above acute exercise interventions, accruing fitness levels have also shown positive outcomes for grey and white matter density and function (Smith et al., 2010; Angevaren et al., 2008; Floel et al., 2010; Hillman et al., 2008; Peters, 2006; Voss et al., 2013). Higher levels of improvements in physical fitness have been related to larger volumes of anterior grey and white matter (Smith et al., 2010; Angevaren et al., 2008; Peters, 2006; Hillman et al., 2008; Floel et al., 2010; Voss et al., 2013).

Frontal Lobe. The frontal lobe is the utmost anterior portion of the brain and contains most of the dopamine-sensitive neurons in the cerebral cortex (Guyton AC, 2011). Dopamine is associated with attention, short term memory tasks, planning, motivation and reward (Guyton AC, 2011; Park \& Reuter-Lorenz, 2009). It assists in limiting and selectively attending to contextual stimuli being delivered to the frontal lobe and thus is correlated to executive function (Guyton AC, 2011; Park \& Reuter-Lorenz, 2009). Dopamine levels are reduced by approximately $10 \%$ per decade of life beginning in early adulthood (Cabeza, Anderson, Locantore, \& McIntosh, 2002). There is a very strong relation between age, the number of dopaminergic receptors, cognition, and motor performance and thus they play a prominent role in certain aspects of cognitive aging (Cabeza et al., 2002; Park \& Reuter-Lorenz, 2009; Voss, Nagamatsu, Liu-Ambrose, \& Kramer, 2011). The frontal lobe along with its dopamine system is 
involved in higher mental functions including: consequence recognition, selection, suppression and mediation of social response or emotion, in addition to distinguishing similarities and differences. The frontal lobe also plays an important role in retaining longer term memories related to emotion (Guyton AC, 2011; Budde et al., 2008; Park \& Reuter-Lorenz, 2009).

Frontal lobe dysfunction due to aging has been related to attention and memory deficits as well as slowing of information processing (Birren \& Fisher, 1995; Peters, 2006). With increasing age, the number of dopaminergic receptors declines and the frontal lobe volume is reduced resulting in impaired cognitive function (Peters, 2006; Cabeza et al., 2002; Voss et al., 2011). The decrement associated with the normative aging process has been noted to be slowed, reduced or even reversed with the inclusion of exercise interventions (Birren \& Fisher, 1995; Cabeza et al., 2002; Myers, 2008; Park \& Reuter-Lorenz, 2009; Peters, 2006; Voss et al., 2011). Exercise has been distinguished to improve aspects of frontal lobe function, including attention and executive function (Barnes et al., 2003; Swardfager et al., 2010; Yu, Ryan, Schaie, Willis, \& Kolanowski, 2009; Teixeira et al., 2012). One mechanism associated with frontal lobe protection is the act of participating in exercise such as walking, running or cycling function (Barnes et al., 2003; Swardfager et al., 2010; Teixeira et al., 2012; Yu et al., 2009). This increases the cerebral blood flow to the frontal lobe resulting in improved executive function. With chronic participation in exercise yielding accrued fitness, frontal lobe activation and function exhibited vast improvements. Conversely, Scherder et.al (2005) demonstrated that six months after an exercise intervention had been removed, the positive cognitive effects did not endure. This finding necessitates for continued, chronic participation in exercise as an inclusionary criterion for lifestyle modification for cognitive health. 
Hippocampus. The temporal lobe is located inferior to the frontal and parietal lobes and houses the hippocampus. The hippocampus possesses the primary role of information consolidation from short term or primary memory to long term or secondary memory as well as spatial navigation, inhibitory processes and verbal memory (Guyton AC, 2011). It is made up of two interlocking components: Ammon's horn and the dentate gyrus (Guyton AC, 2011). The dentate gyrus is of primary interest as it supports neurogenesis in the adult brain (Erickson et al., 2011; Floel et al., 2010; Kim et al., 2010; Pereira et al., 2007; van Praag, 2009). Hippocampal integrity, specifically volume, has been correlated to adverse cognitive modifications related to advancing age (Chaddock et al., 2010; Erickson et al., 2009; McAuley et al., 2011; Voss et al., 2011). The relation of hippocampal shrinkage to cognitive and memory impairment has been well documented delineating that reductions in hippocampal volume result in memory decline, learning aptitude, and overall cognitive function (Erickson et al., 2009; Erickson et al., 2011; Kim et al., 2010; Van der Borght et al., 2007; van Praag, 2009). Because the hippocampus is the primary neural structure that assists in formation of secondary memory, this portion of memory is most affected by volume loss.

Hippocampal volume is reduced by approximately 1-2\% annually in healthy older adults (Erickson et al., 2011). The primary sources of this volume reduction is impedance or retardation of neurogenesis (neuron generation) and neuronal apoptosis (neuron death) due to aging, irradiation and stress (Burns et al., 2008; Erickson et al., 2009; Erickson et al., 2011; Kim et al., 2010; Van der Borght et al., 2007; van Praag, 2009). Buckner (2004) suggests that some brain shrinkage is considered to be a normative part of the aging process, however declining hippocampal volume and stimulation is a subclinical marker for dementia and thus is considered a result of pathological aging. This theory is further supported by the findings that individual 
variations in hippocampal volume and atrophy fluctuate between research participants and lifestyle characteristics (Burns et al., 2008; Erickson et al., 2009; Erickson et al., 2011; Kim et al., 2010; Van der Borght et al., 2007; van Praag, 2009). Buckner's statement supports the proposal that reductions in hippocampal volume and stimulation can be prevented.

Exercise can be viewed as a premier prevention strategy for temporal lobe and hippocampal volume reductions and neuronal apoptosis (Kramer \& Erickson, 2007; Ahamed et al., 2007; Blackmore, Golmohammadi, Large, Waters, \& Rietze, 2009; Chaddock et al., 2010; Burns et al., 2008; Erickson et al., 2009; Erickson et al., 2011; McAuley et al., 2011; Kim et al., 2010; Pereira et al., 2007; Van der Borght et al., 2007; van Praag, 2009; Yu et al., 2009; Zlomanczuk et al., 2006; Leal \& Yassa, 2013). Exercise provides the strongest neurogenic stimulus ultimately resulting in improved cognition (Van der Borght et al., 2007; Ahamed et al., 2007; Blackmore et al., 2009; Chaddock et al., 2010; Burns et al., 2008; Erickson et al., 2009; Erickson et al., 2011; Kim et al., 2010; Kramer et al., 2002; Kramer \& Erickson, 2007; McAuley et al., 2011; Pereira et al., 2007; van, 2009; Yu et al., 2009; Swardfager et al., 2010; Zlomanczuk et al., 2006). It has been noted that exercise elicits great effects on the hippocampus via enhanced electrical activity, neurotransmitter secretion, neurogenesis, blood volume and circulation, brain-derived neurotrophic factor (BDNF) stimulation, angiogenesis (new blood vessel growth), and increasing synaptic plasticity(Van der Borght et al., 2007; Ahamed et al., 2007; Blackmore et al., 2009; Chaddock et al., 2010; Erickson et al., 2009; Erickson et al., 2011; Kim et al., 2010; Kramer et al., 2002; Kramer \& Erickson, 2007; McAuley et al., 2011; Pereira et al., 2007; van Praag, 2009; Yu et al., 2009; Swardfager et al., 2010; Zlomanczuk et al., 2006). Aerobic exercise and training have been the primary focus for improving or maintaining hippocampal integrity. Participation in acute aerobic bouts as well as chronic aerobic 
involvement were both found to be beneficial to hippocampal preservation and development. Erickson et.al. (2011) determined that moderate intensity aerobic exercise can effectively increase the size of the hippocampus, reversing volume loss. The dentate gyrus, or anterior portion of the hippocampus, has been selectively targeted via exercise manifestations resulting in increased volume (Pereira et al., 2007; Ahamed et al., 2007; Floel et al., 2010; Asl, Sheikhzade, Torchi, Roshangar, \& Khamnei, 2008; Kim et al., 2010). This notion of selectivity suggests that aerobic exercise may elicit the largest changes in regions of the brain that express the highest degeneration with age. Newly generated neurons produced by the dentate gyrus in the hippocampus result in improvement or maintenance of learning and memory function (Asl et al., 2008; Floel et al., 2010; Kim et al., 2010; Pereira et al., 2007). Participation in aerobic exercise also promotes angiogenesis resulting in new capillary formation and greater proliferation of the dentate gyrus and circulation to the hippocampus (Asl et al., 2008; Blackmore et al., 2009; Chaddock et al., 2010; Burns et al., 2008; Erickson et al., 2011; Erickson et al., 2009; Floel et al., 2010; Kim et al., 2010; Van der Borght et al., 2007; Kramer \& Erickson, 2007; McAuley et al., 2011; Pereira et al., 2007; van Praag, 2009; Yu et al., 2009; Zlomanczuk et al., 2006; Erickson et al., 2009). Escalation of blood volume is considered to be a correlate of neurogenesis resulting in stimulation of tissue growth (Erickson et al., 2009; Voss et al., 2011). Exercising lab animals have been regularly shown to develop new neurons in the hippocampus, while sedentary animals do not display this trait suggesting that exercise is the key variable necessary for stimulating volume increase (Blackmore et al., 2009; Kim et al., 2010; Van der Borght et al., 2007). Though aerobic training has been the primary focus of study, strength training and combined training (aerobic and strength) have also been observed to improve memory strategies associated with the 
hippocampus (Lachman ME, 2006; Liu-Ambrose et al., 2010; Pontifex, Hillman, Fernhall, Thompson, \& Valentini, 2009).

It is not only the act of participating in exercise that has been proposed as a positive protective mechanism against hippocampal atrophy, but also the resulting gains in fitness. Many studies have observed a substantial difference in hippocampal volume when investigating measurements in cardiorespiratory fitness $\left(\mathrm{VO}_{2}\right)$ (Blumenthal \& Madden, 1988; Burns et al., 2008; Komulainen et al., 2008; Pereira et al., 2007; Swardfager et al., 2010; Van Boxtel et al., 1997). Individuals with greater cardiorespiratory capacity had more substantial progressive changes in hippocampal volume as well as superior spatial memory performance (Asl et al., 2008; Chaddock et al., 2010; Erickson et al., 2009; Kim et al., 2010; Stroth, Hille, Spitzer, \& Reinhardt, 2009). Even after adjusting for potentially confounding lifestyle factors such as age, sex and level of education, aerobic fitness levels were highly associated with preservation of hippocampal volume and spatial memory tasks (Asl et al., 2008; Chaddock et al., 2010; Erickson et al., 2009; Erickson et al., 2011; Kim et al., 2010; Stroth et al., 2009). While many studies report the positive association between cardiorespiratory fitness level and improvement in hippocampal volume and spatial memory, others suggest that higher fitness levels earlier in life, prior to the onset of deterioration, is the primary moderating factor (Middleton et al., 2010; Dik, Deeg, Visser, \& Jonker, 2003; Geda et al., 2010; Wilson et al., 2005). Participation in chronic exercise yielding fitness gains prior to the inception of hippocampal structural decrease is theorized to result in greater hippocampal size (Erickson et al., 2011). The preemptive increase in volume prior to the onset of structural compromise results in the creation of a 'cushion' allowing for volume loss without noticeable decrements in memory performance (Erickson et al., 2011). 
Cerebellum. One of the more inferiorly located portions of the brain, the cerebellum, is involved in fine motor control as well as cognitive functions including: attention, language, and adjustments to sensorimotor relationships (Guyton AC, 2011). The cerebellum is yet another area that is highly affected by the aging process (Floel et al., 2010; Myers, 2008; Park \& ReuterLorenz, 2009). Primary modes of compromise elicited in the cerebellum include neuronal body loss and reduced overall volume (Myers, 2008; Park \& Reuter-Lorenz, 2009). As in previously discussed portions of the brain, exercise can produce protective effects that allow for diminishing cerebellum shrinking and negative structural changes associated with advancing age (Black, Isaacs, Anderson, Alcantara, \& Greenough, 1990; Floel et al., 2010; Isaacs, Anderson, Alcantara, Black, \& Greenough, 1992; Raz N, 2004; Raz \& Rodrigue, 2006). Exercise has been shown to increase localized grey matter volume within the cerebellum as well as inducing angiogenesis which improves overall function (Black et al., 1990; Isaacs et al., 1992; Floel et al., 2010; Raz \& Rodrigue, 2006).

BDNF \& IGF-1. Brain-derived neurotrophic factor (BDNF) is a neurotrophin growth factor that assists in supporting neuroprotection and is a mediator of neurogenesis, dendritic expansion, and differentiation of new neurons and synapses within the central nervous system (CNS) and peripheral nervous system (PNS) (Asl et al., 2008; Erickson et al., 2011; Goekint et al., 2010; Kim et al., 2010; Komulainen et al., 2008; Radak et al., 2010; Voss et al., 2011). It is vital for normal neural development and maintenance throughout the lifespan and plays a key role in modulating synaptic transmission and plasticity (Asl et al., 2008; Erickson et al., 2011; Goekint et al., 2010; Kim et al., 2010; Komulainen et al., 2008; Kramer \& Erickson, 2007; Radak et al., 2010; Voss et al., 2011). BDNF is actively present in the hippocampus, cerebral cortex and basal forebrain (frontal lobe). This secreted protein is imperative for long-term 
potentiation and can affect learning, memory and executive function (Asl et al., 2008; Erickson et al., 2011; Goekint et al., 2010; Kim et al., 2010; Komulainen et al., 2008; Kramer \& Erickson, 2007; Radak et al., 2010; Voss et al., 2011). Insulin-like growth factor 1 (IGF-1) is closely related to BDNF in that it also promotes neuronal growth, survival and differentiation within the CNS and PNS (Asl et al., 2008; Lachman ME, 2006; Liu-Ambrose et al., 2008; Smith et al., 2010). These two substrates provide regeneration for cerebral structures and act as a protectant against cognitive decline (Asl et al., 2008; Erickson et al., 2011; Goekint et al., 2010; Kim et al., 2010; Komulainen et al., 2008; Kramer \& Erickson, 2007; Lachman ME, 2006; Liu-Ambrose et al., 2008; Radak et al., 2010; Smith et al., 2010; Voss et al., 2011). BDNF and IGF-1 concentrations and receptor expression decrease in the hippocampus with increasing age resulting in deleterious effects on memory (Kim et al., 2010; Komulainen et al., 2008; Radak et al., 2010; Voss et al., 2011).

BDNF and IGF-1 can be influenced by many external stimuli including physical activity. It has been clearly shown that circulating BDNF and IGF-1 levels will rise with acute and chronic moderate to high intensity aerobic exercise (Asl et al., 2008; Erickson et al., 2011; Kim et al., 2010; Markowska, Mooney, \& Sonntag, 1998). The increase in circulating BDNF promotes "reversal of synapse breakdown, normalization of aberrant gene expression and, most importantly restoration of memory and learning" (Radak, 2010 pg 778). It is consequently considered to be a critical factor in exercise-induced learning and memory benefits. The primary site for neurogenesis via BDNF interaction is the hippocampus (and dentate gyrus within the hippocampus) which results in an increase in hippocampal volume and improved memory and cognitive function. 
There is support for an interdependent relationship between exercise induced BDNF and IGF-1: if IGF-1 is blocked from entering the hippocampus, the positive effects of these proteins will not occur (Carro, Trejo, Busiguina, \& Torres-Aleman, 2001; Ding, Vaynman, Akhavan, Ying, \& Gomez-Pinilla, 2006). An additional theory is that exercise induced BDNF up regulation occurs because there is an age-related reduction in IGF-1 suggesting that the increase in BDNF is a compensatory mechanism for neural health (Voss, 2011). Regardless of the reasoning for BDNF and IGF-1 proliferation, moderate to intense aerobic exercise and cardiovascular fitness have been associated with improvements in cerebral volume, memory and cognition (Asl et al., 2008; Erickson et al., 2011; Goekint et al., 2010; Kim et al., 2010; Komulainen et al., 2008; Kramer \& Erickson, 2007; Lachman ME, 2006; Liu-Ambrose et al., 2008; Radak et al., 2010; Smith et al., 2010; Voss et al., 2011). Interestingly, Goekint et.al. (2010) reported that eight weeks of training showed improvements in circulating BDNF in rodents but after an eight week detraining period BDNF levels were decreased even lower than the sedentary control group. This finding raises great interest in the effects of both training and detraining on cerebral structures.

\section{Cognitive Risk Factors}

The strongest risk factors for cognitive decline and mild cognitive impairment are increasing age and possession of a genetic factor known as APOE-e4 (Bates et al., 2009a; Birren \& Fisher, 1995; Bielak, 2010; Deeny et al., 2008; Etnier, Nowell, Landers, \& Sibley, 2006; Hultsch, Hertzog, Small, \& Dixon, 1999; Myers, 2008; Peters, 2006). Many other lifestyle factors and medical conditions have been linked to cognitive deterioration, these include: diabetes, smoking, cardiovascular disease, hypertension (high blood pressure), cerebrovascular disease, depression, hypercholesterolemia (elevated cholesterol), sedentary lifestyle, and 
irregular participation in mentally or socially stimulating activities (Strength of the relation between the risk factor and cognitive impairment is depicted in Table 2.1) (Baker et al., 2010; Barnes et al., 2003; Myers, 2008; Small et al., 2006; Chuang et al., 2013). The existence of risk factors for cerebral dysfunction is further aggravated by the effects of the aging even for individuals that are under the age of 65 (Birren \& Fisher, 1995). Many of these lifestyleassociated risk factors are interrelated and interdependent. Though increasing age and genetic predisposition may not be modifiable, the majority of the other risk factors listed can be curtailed or even eliminated with the inclusion of regular physical activity. It is important to understand the potential effect on cognitive impairment for these confounding variables as they will be a commonality amongst the aging population that will be addressed in this project.

Table 2.1: Major risk factors for CVD and their association with Cognitive Decline or AD

\begin{tabular}{|c|c|}
\hline Risk Factor & $\begin{array}{l}\text { Evidence for relation with cognitive decline or } \\
\qquad A D\end{array}$ \\
\hline CVD & **** \\
\hline HTN & $* *$ \\
\hline Lipids & $* *$ \\
\hline Type 2 diabetes & $* * *$ \\
\hline Inflammation & * \\
\hline
\end{tabular}

CVD, cardiovascular disease; AD, Alzheimer's disease; ***strong evidence; **moderate evidence; *weak evidence Modified from Stampfer 2006

Type 2 Diabetes. Type 2 diabetes is a condition where glucose accumulates in the blood instead of entering the cells to be used as fuel due to a relative insulin deficiency (peripheral tissue insulin resistance or defective insulin secretion). This increase in glucose is known as 
hyperglycemia which is commonly linked to inflammation in the blood vessels and can result in altered blood flow to the cerebral structures as well as other areas of the body (Baker et al., 2010). Diabetes, specifically type 2 diabetes, has been shown to result in memory loss, depression and other types of cognitive impairment (Baker et al., 2010; Novak V, 2011). Novak and colleagues (2011) discovered that two adhesion molecules: soluble intercellular adhesion molecule (sICAM) and soluble vascular adhesion molecule (sVCAM), specific to older adults with type 2 diabetes, cause inflammation in the brain that affect the blood vessels. These markers for endothelial integrity altered cerebral vasoreactivity ultimately resulting in poorer circulation in various parts of the brain ultimately resulting in brain tissue atrophy within the frontal and temporal lobes (Novak, 2011). As noted previously, frontal and temporal brain regions are paramount to executive functioning and generalized cognition and are also targeted by accruing age as well as type 2 diabetes pathophysiology. Low circulating BDNF levels have likewise been observed in individuals with type 2 diabetes (Baker et.al, 2010).

Individuals with type 2 diabetes do not experience a normalized response to exercise in relation to hormonal and metabolic events necessary for glucose homeostasis (Farrell, 2011; 2010). Though every person with this condition will react differently to exercise, a few common links can be noted: (1) possible improvement in blood glucose control, (2) improved insulin sensitivity, (3) reduction in body fat, (4) cardiovascular benefits, (5) stress reduction, and (6) prevention of type 2 diabetes (Farrell, 2011; 2010). Improvements in blood glucose control and insulin sensitivity are interconnected. Exercise training results in improved insulin sensitivity that culminates in an overall ability for the body to use circulating glucose thus improving blood glucose control. Weight loss also increases insulin sensitivity providing the same benefits of blood glucose control. The risk of cardiovascular disease in individuals with type 2 diabetes is 
2-4 times higher than that of individuals without the disease (American Heart Association, 2012). It is therefore very important for individuals with type 2 diabetes to improve cardiovascular health to assist in reducing this risk. Stress from illness, physical or mental stimuli elicits a disruptive response via increasing counter-regulatory hormones, ketones, free fatty acids, and urine output. Ultimately, this cascade of events causes rise in blood glucose levels creating further disturbance to an already compromised system (Atalay M \& DE Laaksonen, 2002; Atalay M \& DE Laaksonen, 2002). Though exercise is a form of physical stress, exercise training actually provides a protective mechanism against the stress response (Atalay M \& DE Laaksonen, 2002).

Recommendations for exercise for individuals with diabetes must be tailored to the participant due to the individualized nature of the disease. General guidelines for exercise prescription suggest inclusion of aerobic exercise, strength and/or anaerobic training, flexibility and functional exercises to mimic activities of daily living (Farrell, 2011; 2010). These recommendations relate to the specific needs of the entire physiological system including those related to executive function and memory. Aerobic exercise has been shown to have positive effects on cognitive and executive function commonly compromised by type 2 diabetes (Baker et al., 2010; Novak V, 2011).

Cardiovascular Disease and Hypertension. Cardiovascular disease (CVD) pertains to any disease of the heart and blood vessels. It is the leading cause of death in Western countries and accounts for one out of every three deaths in the United States (Centers for Disease Control and Prevention, 2012). Hypertension (HTN), or high blood pressure, is a type of cardiovascular disease that is commonly referred to as the "silent killer" due to the non-symptomatic nature of the dysfunction. Even though HTN is a type of CVD, it is still considered to be one of the 
primary risk factors for other types of CVD (Farrell, 2011; 2010). Hypertension is a chronic pressure increase within the blood vessels applying excess stress and burden on the vascular system resulting in chronic damage do the vasculature. Ultimately, this condition can result in myocardial infarctions (heart attack), cerebral vascular accidents (stroke), aneurysm, heart failure, kidney dysfunction, vision loss, metabolic syndrome as well as disturbances in cerebral aging, executive function and memory (Bates et al., 2009a; Clarkson-Smith \& Hartley, 1990; Myers, 2008; Small et al., 2006).

A strong link has been associated between CVD, as well as CVD risk factors, and cognitive impairment, primarily Alzheimer's disease (AD) (Martins et al., 2006; Masel, Raji, \& Peek, 2010). Lifestyle factors are a large component of this connection as the contributing factors parallel one another. The common outcome from poor lifestyle choices resulting in CVD or HTN has been shown to lead to compromised cerebral vasculature (Bates et al., 2009a; Clarkson-Smith \& Hartley, 1990; Luchsinger, Tang, Stern, Shea, \& Mayeux, 2001; Masel et al., 2010; Scullin et al., 2013). CVD has also been associated with over-activity of the sympathetic nervous system reducing the neural control of the circulation and promoting greater flux in blood pressure (Mueller, Kiesewetter, Binder, Beckmann, \& Dittrich, 2007). Selective losses of frontal lobe grey and white matter and decline in hippocampal density have also been associated with CVD and HTN (Raz \& Rodrigue, 2006; Raz, Rodrigue, \& Acker, 2003; Scullin et al., 2013). Three possible mechanisms have been suggested for the influence of vascular disease on executive function: (1) CVD and dementia interdependently share the same risk factors, thus the rate for occurrence of both is amplified, (2) vascular damage perpetuated by CVD predisposes neurodegeneration, or (3) vascular factors associated with CVD directly cause neuronal death and accumulation of plaques and tangles (Myers, 2008; Stampfer, 2006; Chuang et al., 2013). 
Studies investigating the relation of cognitive performance, particularly executive function, to CVD and HTN suggest that cognition is associated with cardiovascular fitness thus participation in aerobic exercise is hypothesized to play a protective role (Bates et al., 2009a; Bates et al., 2009b; Clarkson-Smith \& Hartley, 1990; Raz \& Rodrigue, 2006; Raz et al., 2003; Scullin et al., 2013).

Recommendations for exercise within this population comprise the inclusion of largemuscle aerobic activity as well as low resistance strength training (Farrell, 2011; 2010). The ultimate goal of the exercise prescription is to increase endurance, control blood pressure, improve strength and advance aerobic capacity (Farrell, 2011;2010). The participation in exercise promotes a downward shift in blood pressure after the training bout, reducing the overall vasculature pressure. This acute response can become chronic with the incorporation of regular training and improved cardiovascular health. Ultimately, the improvement in the vascular structures yields greater circulatory capacity impeding or reversing the damaging effects to the cerebral system and impeding executive function (Bates et al., 2009a; Clarkson-Smith \& Hartley, 1990; Myers, 2008; Raz \& Rodrigue, 2006; Raz et al., 2003).

Cerebrovascular Disease. Cerebrovascular disease is a predominately lifestyle-related disease that is hallmarked by hardening of the arterial walls within the brain (Farrell, 2011; 2010). Hardening of the vessels restrict their capability to contract and expand with increased or reduced blood flow producing impairment in overall blood supply to the affected structures. Cerebrovascular disease is a canopy term for a grouping of conditions including: cerebrovascular accidents (CVA or stroke), carotid stenosis, vertebral stenosis, intracranial stenosis, aneurysms and other vascular malformations (Farrell, 2011; 2010). As in other vascular diseases, common 
risk factors include: smoking, hypertension, diabetes, hypercholesterolemia, and cardiovascular disease (Centers for Disease Control and Prevention, 2012).

Cerebrovascular disease is thought to be one of the primary underlying causes of dementia and mild cognitive impairment (Bates et al., 2009a; Carles S Jr et al., 2007; ClarksonSmith \& Hartley, 1990; Raz \& Rodrigue, 2006). Though the ability to link the two is currently challenging, correlation has been widely observed (Bates et al., 2009a; Clarkson-Smith \& Hartley, 1990; Raz \& Rodrigue, 2006; Raz et al., 2003). Both infarctions due to vascular frailty and alterations in white matter predominately occurring in the frontal lobe are predicted to be the primary determinants affecting executive function impairment due to cerebrovascular disease (Baker et al., 2010; Barnes et al., 2003; Birren \& Fisher, 1995; Green, Maiorana, O'Driscoll, \& Taylor, 2004; Lautenschlager et al., 2008; Peters, 2006; Radak et al., 2001; Small et al., 1997; Goh, Beason-Held, An, Kraut, \& Resnick, 2013). As described above, exercise has the ability to protect or reverse volume losses within the frontal lobe function (Barnes et al., 2003; Birren \& Fisher, 1995; Cabeza et al., 2002; Myers, 2008; Park \& Reuter-Lorenz, 2009; Peters, 2006; Teixeira et al., 2012; Voss et al., 2011; Yu et al., 2009). It is also noted that aerobic training can substantially alter vascular health throughout the body, manifesting a higher ability to support circulation and reduce vascular accidents (Baker et al., 2010; Barnes et al., 2003; Birren \& Fisher, 1995; Green et al., 2004; Lautenschlager et al., 2008; Peters, 2006; Radak et al., 2001; Small et al., 1997).

Hyperlipidemia \& Hypercholesterolemia. Hyperlipidemia is a condition where the triglycerides and cholesterol in the blood are elevated while hypercholesterolemia refers to elevated blood cholesterol concentrations. Both imply greater risk for vascular function and are therefore considered to be risk factors for reduced cognitive function (Kantor, Cullinane, Sady, 
Herbert, \& Thompson, 1987; Kim et al., 2010; Radak et al., 2001; Whelton, Chin, Xin, \& He, 2002). Findings indicate that enhanced cholesterol may play a role in the development of AD, inflammatory responses as well as working memory errors (Ullrich, Pirchl, \& Humpel, 2010). A negative association between high density lipoproteins (good cholesterol promoted by exercise) and Amyloid- $\beta$ protein (a protein responsible for age-related memory decline) has been supported (Bates et al., 2009b; Lesne \& Kotilinek, 2005; Westerman et al., 2002; Janus et al., 2000). Some studies discuss the plausibility of these factors having a greater protective effect on cognitive function during midlife versus closer to the onset of the disease (Goldstein et al., 2008). Regardless of onset, reducing the risk of hyperlipidemia and hypercholesterolemia could be a preemptive strategy to contest against reductions in executive function. Exercise has been shown to reduce the risk of contracting these conditions as well as decreasing the effects that these disorders cause on physiological systems (Madden et al., 2009).

Cerebral Structure Conclusions. Though there are conflicting findings for many of these risk factors, there is a strong body of evidence supporting the belief that these factors, independently or cumulatively work against cognitive function. It is vital to understand the relation between these confounding variables and how they respond to exercise in order to comprehend what occurs with exercise intervention.

\section{Normative Physiological Response of Land Exercise Related to Executive Function}

The means by which the body reacts to exercise is dependent on a variety of factors including frequency, duration, mode, intensity, and environmental conditions. In general, the human body utilizes a variety of somatic systems to produce and support the act of exercise. Irrespective of the type of exercise being performed, the body is required to produce select muscular contractions in order to yield coordinated movement. For the movement to occur 
successfully, the body requires greater oxygen and skeletal muscle substrates as well as removal of metabolic wastes and carbon dioxide. Metabolic, cardiovascular, nervous, endocrine, gastrointestinal, musculoskeletal, and respiratory responses and mechanisms are required to work cohesively to produce an intrinsic environment to permit the continuation of gross motor movement (Farrell, 2011; 2010). With the creation of this environment, a cascade of reactions occurs to further allow adaptation of the somatic systems involved. These reactions can influence not only physical functioning of the exercising body, but cognitive operation as well.

Though the body's systems are highly interdependent and interregulated, there are some systems that have a greater impact on cognitive stability, maintenance and improvement than others. The neurological, metabolic, and cardiovascular/circulatory systems are the most impending body systems during exercise that are related to executive function. They are theorized to promote cerebral adaptation by stimulating the growth of new circulatory structures (arteries, arterioles, and capillaries) that further encourage the creation and deposition of specified cerebral tissues (Kramer et al., 2002; Studenski et al., 2006). Support for physical activity-dependent CNS plasticity resulting in changes in the number, structure and function of neurons exist throughout the literature (Adlard, Engesser-Cesar, \& Cotman, 2011; Asl et al., 2008; Floel et al., 2010; Galvan \& Jin, 2007; Hultsch et al., 1999; Kim et al., 2010; Komulainen et al., 2008; Kramer \& Erickson, 2007; Peters, 2006; van Praag, 2009; Voss et al., 2011).

Nervous System. The primary role of the nervous system is to define how accurately the body generates, responds to and adapts to movement acutely and chronically. It reacts to short bouts of exercise and also allows for adaptation to repetitive movements and chronic training (Farrell, 2011; 2010). Neural control mechanisms determine muscular recruitment via activation of motor units which inherently govern intensity and endureability of the movement being 
produced (Farrell, 2011; 2010). Aside from determination of movement, the muscular properties including size, speed and fatigability are also highly controlled by the nervous system. Along with movement, learning and adaptational memory, via synaptic modifications in response to stimuli, are attributed to the nervous system, particularly the spinal cord and hippocampus (Asl et al., 2008; Erickson et al., 2009; Erickson et al., 2011; Ke et al., 2011; Kim et al., 2010; Kramer \& Erickson, 2007; Myers, 2008; Peters, 2006; Radak et al., 2010; Smith et al., 2010; Van der Borght et al., 2007; van Praag, 2009; Voss et al., 2011; Zec, 1995).

Dynamic exercise has been purported to enhance recovery from neurotraumatic injuries, enhance learning and stimulate neurogenesis (Blackmore et al., 2009; Deeny et al., 2008; Erickson et al., 2009; Floel et al., 2010; Galvan \& Jin, 2007; Hillman et al., 2009; Hillman et al., 2003; Kim et al., 2010; Pereira et al., 2007; Van der Borght et al., 2007; van Praag, 2009). Though the literature has not found a conclusive reason for these positive outcomes, researchers speculate that physiological changes in the nervous system including excitation and inhibition of action potentials, synaptic effectiveness of neural connections, synchronization, neurodegenerative protection, increase in BDNF as well as BDNF mRNA and genes linked to BDNF, and other neurotrophic growth factors probably pose the greatest impact on the adaptive processes of the nervous system as linked to exercise (Blackmore et al., 2009; Deeny et al., 2008; Erickson et al., 2009; Floel et al., 2010; Galvan \& Jin, 2007; Hillman et al., 2009; Hillman et al., 2003; Kim et al., 2010; Pereira et al., 2007; Van der Borght et al., 2007; van Praag, 2009).

Metabolic System. The metabolic system consists of all of the chemical reactions and substrate breakdown that take place within the body that allow for physical changes, continued growth, overall function and sustainment of life. Metabolism can be broken down into 2 subcategories: catabolism, which is breakdown or degradation; and anabolism, which is growth, 
repair or construction. There are three primary energy systems that make up the metabolic system: (1) phosphagen / ATP-PC, (2) glycolytic, and (3) oxidative (Farrell, 2011; 2010). Though all three sources will supply a portion of the energy need at all times, one system will predominate depending on the physiological demands placed on the body. Exercise duration and intensity are the two primary stimuli that determine which energy system will be utilized within the body's tissues and this will predispose selectivity of energy substrates (protein, carbohydrate and fat). Though the body can utilize all three energy sources and systems, the brain's specified tissue can only exploit glucose and the lactate byproduct as its energy substrate (Farrell, 2011; 2010). The cerebral tissue receives $25 \%$ of total body glucose utilization and $20 \%$ of total body oxygen consumption from the arterial blood (Magistretti et al., 1994; Pellerin et al., 1998). Variations in energy metabolism within regions of the brain differ with functional activity and it has been noted that changes have been observed in regional metabolism with cerebral diseases such as AD, Huntington's and Parkinson's (Herholz, 2003).

It is necessary for the brain to retain a continuous supply of oxygen and glucose in order for continued function; this is known as cerebral autoregulation. Because the brain has virtually no glucose storage capacity, if the brain's nutrient supply via the bloodstream is hindered for as little as six seconds, consciousness is impaired or lost (Guyton AC, 2011). This is one of the primary reasons that the cerebral tissue retains priority to cardiac output during normal and exercising conditions. The cerebral blood flow response varies from submaximal to maximal exercise intensities. During submaximal exercise, cardiac output is increased resulting in an increase in blood flow to the brain. The excess blood flow highly increases oxygen delivery capacity resulting in excess oxygen availability beyond the brain's needs. Maximal exercise, however, results in impairment of cerebral autoregulation leading to a deficiency in blood supply 
to the brain resulting in oxygen and glucose decrements. During resting conditions, the brain's energy is provided by glucose with some available compensation from lactate. The compensatory utilization of lactate as energy is remarkably increased during exercise by up to $33 \%$ of total energy demand (Farrell, 2011; 2010). This increase in lactate utilization is dependent upon exercise intensity; the more intense the exercise, the greater the need for lactate compensation (Farrell, 2011; 2010).

Aerobic and anaerobic exercise training both lead to modifications in the metabolic system (Farrell, 2011; 2010). Substrate availability and usability are altered with chronic training leading to effects on cognitive function (Baker et al., 2010; Herholz, 2003; Kim et al., 2010; Pereira et al., 2007; Small et al., 2006). Without the appropriate fuel, the brain is unable to elicit applicable executive function. Exercise induced cognitive effects have been linked to improvements in energy metabolism and insulin signaling within the brain when a six month aerobic training regimen was imposed upon the participants (Baker et al., 2010).

It has been noted in the research that exercise training moderates exercise induced decreases in insulin and increases in counterregulatory hormones (Kim et al., 2010; Baker et al., 2010; Herholz, 2003; Pereira et al., 2007; Small et al., 2006; Small, Dixon, McArdle, \& Grimm, 2012). This holds a level of importance because it has been shown that when exposed to an exercise intervention, individuals with glucose intolerance displayed important gains in executive function (Baker et.al, 2010). The stipulation regarding exercise is that the training effects on insulin sensitivity are complete less than 2 weeks after the onset of training and lost less than 2 weeks after training ceases so continuous training is necessary for sustained effects (Baker et.al, 2010). 
Cardiovascular System. The cardiovascular system is comprised of the heart and vasculature necessary to distribute blood and all of the nutrients it carries throughout the body. This system is paramount for delivery and removal of metabolic constituents necessary for cerebral and somatic function. A series of blood vessels are networked throughout the brain to supply the neural tissue with oxygenated blood, glucose and other nutrients while removing metabolic byproducts (Guyton AC, 2011). As mentioned previously, the brain possesses many safeguards to allow for cerebral autoregulation in accordance with maintaining functional status, the cardiovascular system is a large contributor to this process. It is an extremely adaptive system with the ability to construct new vasculature in order to meet the demands placed on the body in a more efficient manner. Exercise promotes this adaptive process by challenging the dynamic capabilities associated with the heart and vasculature. During exercise, heart rate increases progressively in accordance with workload while the volume of blood pumped by the heart (stroke volume) increases the cardiac output or blood flow to the systemic circulation. This process is mediated by metabolic demand and aided heavily by the constrictive and dilative properties of the vessels, particularly the arteries. Vasculature within areas in need of greater blood supply will dilate to allow for a more unobstructed, open flow to the area, while areas that do not need as much blood will constrict to further support the redistribution of blood to the targeted areas of need (Farrell, 2011).

Cerebral circulation, as mentioned previously, is substantially affected by exercise and intensity of the activity (Barnes et al., 2003; Swardfager et al., 2010; Teixeira et al., 2012; Yu et al., 2009). Other confounding factors that regulate cerebral blood flow include blood viscosity, blood vessel radius, cerebral perfusion pressure (the net pressure of blood flow to the brain), and blood pressure. Exercise can enhance the vasculature's ability to adjust to environmental 
changes due to 'training' the cardiovascular system to become more dynamic (Farrell, 2011).

The necessary variation of vessel dilation and constriction in order to maintain compulsory cerebral blood perfusion during fluctuating exercise 'teaches' the system to adjust swiftly and efficiently when changes arise. The need for chronic increases in blood flow, as in the case with exercise intervention, engages new vasculature to be formed within the structures being employed, a response termed angiogenesis (Farrell, 2011). This phenomenon occurs throughout the body's systems, including the musculature and cerebral tissue. Angiogenesis has been linked to improved learning and memory and seems to be dominating in areas that are stimulated and recruited during the exercise bout. It is primarily mediated by insulin-like growth factor 1 (IGF1) which is mediated by vascular endothelial growth factor (VEGF) (Asl et al., 2008; Erickson et al., 2011; Goekint et al., 2010; Kim et al., 2010; Kramer \& Erickson, 2007; Komulainen et al., 2008; Lachman ME, 2006; Liu-Ambrose et al., 2008; Radak et al., 2010; Smith et al., 2010; Voss et al., 2011). Increased circulation and secretion of both substrates are enhanced by exercise further linking exercise to positive structural changes within the brain.

Somatic Systems Conclusion. The body systems related to cognition are acutely affected by a single bout of exercise and exhibit chronic effects as a result of continuous exercise training. Primary alterations credited to participation in exercise exhibited in the body correlating to improvements in executive function include increased cerebral circulation and increased vascularization (Asl et al., 2008; Erickson et al., 2011; Goekint et al., 2010; Kim et al., 2010; Kramer \& Erickson, 2007; Komulainen et al., 2008; Lachman ME, 2006; Liu-Ambrose et al., 2008; Radak et al., 2010; Smith et al., 2010; Voss et al., 2011). These primary exerciseinduced adaptations are recognized to result in deposition of new neuronal structures as well as 
differentiation of those structures within targeted areas of the brain ultimately leading to improved cognitive performance and executive function.

\section{Effects of Exercise Modality on Executive Function}

There are a wide variety of exercise modalities that individuals can participate in to glean fitness benefits. These modalities can be divided into two primary categories: aerobic exercise and anaerobic exercise. Aerobic training consists of activities utilizing large muscle groups to produce continuous, rhythmic movement that can be sustained over a longer period of time. It is dependent on aerobic metabolic systems that require large amounts of oxygen to produce the energy necessary for sustaining movement. These activities are usually of longer duration and performed at low to moderate intensity. Anaerobic exercise, in contrast to aerobic exercise, comprises faster, higher intensity movements such as weight training or sprinting. It is primarily reliant on metabolic systems that do not require great amounts of oxygen in order to break-down and supply the working tissues with energy. Both types of exercise are widely accepted, generalized modes of exercise training for improvement of fitness but have varied reactions on

physiological stress resulting in different acute and chronic physiological responses. This is why it is necessary to discuss the two types and the current research findings pertaining to executive function.

Aerobic Exercise. Aerobic training has been noted to have both acute and chronic effects on various components of executive function (Liu-Ambrose et al., 2010; Baker et al., 2010; Barnes et al., 2003; Bielak, 2010; Budde et al., 2008; Davis et al., 2007; Davis et al., 2007; Davis et al., 2008; Liu-Ambrose et al., 2008; Swardfager et al., 2010; Teixeira et al., 2012; Hogan et al., 2013; Joyce et al., 2014). Research supports the link between participation in chronic aerobic exercise and: improvements in spatial learning, memory, cognitive capacity, 
selective and divided attention, working memory, cognitive flexibility, planning, inhibition, decision making, problem solving, cognitive speed, and overall cognitive function (Angevaren et al., 2008; Baker et al., 2010; Barnes et al., 2003; Birren \& Fisher, 1995; Budde et al., 2008; Fabre et al., 2002; Hillman et al., 2009; Hillman et al., 2008; Hillman et al., 2003; Ke et al., 2011; Liu-Ambrose et al., 2010; Man et al., 2010; Scherder et al., 2010; Smith et al., 2010; Van der Borght et al., 2007; Teixeira et al., 2012; Yaguez et al., 2011). Aerobic training has also been correlated with increased cerebral electrical activity and neurotransmitter secretion as well as reductions in neural apoptosis, increased growth factor modulation, increased vascularization, declines in brain volume loss, and memory loss (Asl et al., 2008; Baker et al., 2010; Berkman et al., 1993; Blumenthal \& Madden, 1988; Burns et al., 2008; Chaddock et al., 2010; Chan et al., 2005; Clarkson-Smith \& Hartley, 1989; Deeny et al., 2008; Erickson et al., 2011; Erickson et al., 2009; Fabre et al., 2002; Floel et al., 2010; Goekint et al., 2010; Hillman et al., 2009; Hillman et al., 2008; Ke et al., 2011; Kim et al., 2010; Klusmann et al., 2010; Komulainen, 2010; Komulainen et al., 2008; McAuley et al., 2011; Nation et al., 2011). The effects of a single bout of exercise have also been studied. Findings support neuropsychological improvements including attention, processing speed, concentration and overall cognitive function (Budde et al., 2008; Carles S Jr et al., 2007; Coles \& Tomporowski, 2008; Goekint et al., 2010; Hillman et al., 2009; Hillman et al., 2008; Hillman et al., 2003; Pontifex et al., 2009). The acute and chronic effects of aerobic training are widely documented and provide some insight linking the effects of exercise with executive function. The overall outcome of exercise is enhanced immune condition of the brain and its structures that results in improved neural plasticity. Though the majority of the studies that have been performed exhibit positive results associated with participation in exercise or accrual of cardiovascular fitness, there are some studies that do not 
display any variations in executive function with the addition of physical activity. These studies may not provide supporting evidence that aerobic training results in positive modifications in cognitive processes; however, they do not illustrate any decrements (table 2.2).

\section{Table 2.2: Physiological and Performance Changes Associated with Aerobic Exercise and Cardiovascular Fitness}

\begin{tabular}{|c|c|}
\hline $\begin{array}{l}\text { Physiological Changes linked to Aerobic } \\
\text { Exercise and CV Fitness }\end{array}$ & $\begin{array}{c}\text { Cognitive Performance Changes linked to } \\
\text { Aerobic Exercise and CV Fitness }\end{array}$ \\
\hline Increased Electrical activity in the brain & Improved Spatial memory \\
\hline Decreased circulating $A \beta$ (biomarker for AD) & Improved Learning \\
\hline Improved glucose regulation & Improved Memory \\
\hline Increased brain plasticity & Improved Cognitive Function \\
\hline $\begin{array}{l}\text { Increased expression of genes encoding } \\
\text { neurotrophins }\end{array}$ & Improved Short-term cognitive capacity \\
\hline Increased neurogenesis\& synaptic connectivity & Improved Selective attention \\
\hline Increased BDNF and other growth factor levels & Improved divided attention\& attentional control \\
\hline Increased task-related brain activity & Improved cognitive flexibility \\
\hline $\begin{array}{l}\text { Increased stimulation and regeneration of } \\
\text { endogenous stem/progenitor cells }\end{array}$ & Improved working memory \\
\hline Increased angiogenesis/vascularization & Reduced decline in problem solving \\
\hline $\begin{array}{l}\text { Increased brain volume (particularly in the } \\
\text { hippocampus, white matter, grey matter, frontal } \\
\text { lobe, cerebellum, temporal lobe) }\end{array}$ & $\begin{array}{l}\text { Increased Psychomotor Speed / Reduced response } \\
\text { slowing }\end{array}$ \\
\hline \multicolumn{2}{|l|}{ Increased structural protection } \\
\hline \multicolumn{2}{|l|}{ Reduction in peripheral and central inflammation } \\
\hline \multicolumn{2}{|l|}{ Reduced oxidative stress } \\
\hline Increased blood flow and volume & \\
\hline
\end{tabular}


The level of cardiovascular (CV) fitness that an individual possesses may play an integral role in attenuating losses in executive function. Research has been completed where levels of physical fitness or physical performance tests were measured then compared to cognitive parameters. These studies propose that baseline CV fitness may be allied with cognitive preservation (Barnes et al., 2003; Barnes et al., 2007; Blumenthal \& Madden, 1988; Burns et al., 2008; Chaddock et al., 2010; Chaddock, Hillman, Buck, \& Cohen, 2011; Erickson et al., 2009; Erickson et al., 2011; Etnier et al., 2006; Hillman et al., 2009; McAuley et al., 2011; Pereira et al., 2007; Scherder et al., 2010; Swardfager et al., 2010; Van Boxtel, Langerak, Houx, \& Jolles, 1996; Yu et al., 2009). This may be due to the reduction in health-related risk factors that can predispose individuals to cognitive dysfunction, reductions in brain atrophy or positive alterations in cognitive processing speed (Bates et al., 2009a; Birren \& Fisher, 1995; Myers, 2008; Peters, 2006; Radak et al., 2010; Swardfager et al., 2010; Teixeira et al., 2012). These findings were further supported by a correlation between cerebral preservation and lifelong participation in CV exercise when compared to their sedentary counterparts (Barnes et al., 2003; Erickson et al., 2009). Long term physical activity has also been noted to result in a $20 \%$ reduction in cognitive decline risk in older women (Weuve et al., 2004). Other studies report that improvements in aerobic fitness precede positive alterations in cognitive capacity (Angevaren et al., 2008). These studies allow extrapolation towards the concept that physiological changes resulting from improved CV fitness (heart rate, vasculature activity, blood pressure) may hold a particular level of importance when clarifying the relation between exercise and executive function. Another subset of aerobically based fitness studies support the notion that participation in physical activity early in life, between the ages of $15-25$, is positively associated with a reduction in loss of cognitive function and performance with the strongest 
relation being reported as teenagers (Bielak, 2010; Denkinger et al., 2012). Though there are many studies that suggest that CV fitness is necessary for cognitive improvement, some studies found that increases in physical activity without $\mathrm{CV}$ benefit also benefit executive function (Benloucif et al., 2004; Floel et al., 2010). These effects may be mediated via metabolic or neurotrophic factors that are tempered by the act of physical activity over the development of CV fitness. Increased arousal state, as determined by cerebral imagery scanning, may also be primary grounds for improvements in executive control (Hillman et al., 2008; Hillman et al., 2009; Voss et al., 2010; Voss et al., 2011). In addition, previously active individuals that resumed aerobic exercise enhanced the memory shielding capacity provided by exercise (Hillman et al, 2008; Voss et al, 2010). In conjunction with the concept of the ability to build on pre-existing or former fitness levels, findings have supported that the acute effects of exercise training are further augmented by chronic exercise training (Denkinger et al, 2012). Thus, the improvements in cognitive function, attention, processing speed and concentration noted to occur following an acute bout of exercise are enhanced with existing chronic training habits.

Because participation in physical activity has such a wide variety of elicited responses, it is a possibility that both physiological and psychological mechanisms work cohesively to promote an environment that preserves or enhances mental function. Stress, psychological wellbeing and improved quality of life are all attributes associated with taking part in exercise. These psychological variables can assist in creating an internal environment that is more inclined to physiological alterations.

Anaerobic Exercise. Anaerobic exercise in the form of strength training or short, intense bouts of exercise has not been a primary focus of study in relation to executive function. There are a limited number of studies that have addressed the use of this modality for cognitive 
adaptations. In the case of aerobic training, there has been a strong case made for its physiological and psychological contribution to the betterment of the cerebral structure and function. This is not the case for anaerobic training. Chronic anaerobic training literature has reported no statistically significant effects on neurocognitive function with varied levels of intensity (Liu-Ambrose et al., 2010; Pontifex et al., 2009). However, acute effects of anaerobic training (effects noted during or immediately following the training stimulus) have reported increased levels of BDNF, epinephrine and norepinephrine, increased speed and improved reaction time as well as working memory span, response inhibition, improved recall, accuracy and learning efficiency (Lachman ME, 2006; Liu-Ambrose et al., 2010; Pontifex et al., 2009; Joyce et al., 2014). One of the fundamental trends found with the acute effects of anaerobic training utilizing weight training or muscular contractions is the concept of the inverted-U relationship to tension and cognition (Tomporowski PD, 1986). The inverted-U refers to the indication that the intensity of muscle contraction elicited, directly affects the cognitive facilitation ability. Thus, if the contraction being produced is low or high, cognitive is low, but if the muscular contraction is of moderate intensity, the resulting cognitive improvements are meaningfully enhanced (Tomporowski PD, 1986).

A recent study conducted by Liu-Ambrose et al. (2010) compared the effect of a once or twice weekly strength training program with that of a balance and toning program on the executive function performance. The 52 week program concluded that the resistance program improved selective attention and conflict resolution as well as whole brain volume and gait speed in comparison to the balance and toning program (Liu-Ambrose et al, 2010). Though strength training and short, intense bouts of physical activity are grouped as anaerobic activities, there are major variations in research outcomes when connected to executive function. A study by Winter 
and colleagues (2007) considered the differences between an intense sprinting group, a moderate aerobic exercise group and a non-exercising control group. The findings from this study revealed a statistically significant variation in learning speed across the differing conditions with the intense running cohort displaying the fastest learning speeds, accelerated by $20 \%$, over the sedentary group immediately following the training sessions (Winter et al., 2007). These results did not continue to hold at the one week or 8 month retest periods. Response times were also faster in comparison to the moderate and control groups immediately following the exercise bout (Winter et al., 2007).

Combined Training. According to a meta-analytic study conducted by Colcombe and Kramer (2003, pg128), "participants in combined strength and aerobic training regimens improved to a reliably greater degree than those in aerobic training alone." This finding may be due to the physiological variations in stimuli resulting in a more diverse manifestation of cognitive improvements (Colcombe \& Kramer, 2003). Improvement in fitness parameters including: strength, range of motion, and $\mathrm{VO}_{2 \max }$ can be achieved with both resistance training as well as aerobic training (Farrell, 2011; Komulainen, 2010). Exercise intensity is one of the primary driving forces necessary to influence many fitness constituents. Thus, in order to improve an individual's level of fitness they must exercise at a specific heart rate, $\mathrm{VO}_{2}$, or rate of perceived exertion in order to promote a higher threshold of training capability. This concept is important when discussing memory because it has been indicated that improvement in fitness parallels improvements in memory function (Angevaren et al., 2008; Coe, Pivarnik, Womack, Reeves, \& Malina, 2006; DiLorenzo et al., 1999; Fabre et al., 2002; Komulainen, 2010; Kwak et al., 2009). Fitness improvement may also be more achievable if individuals participate in multi- 
modal forms of exercise; resistance training with aerobic training, or sprinting with moderate aerobic training.

Exposure to various types of training may also influence overall activity level throughout the lifespan (Perrig-Chiello, Perrig, Ehrsam, Staehelin, \& Krings, 1998). Perrig-Chiello and colleagues (1998) conducted an intervention where the experimental group was instructed on resistance training exercises while the control was not. One year after the intervention, they reassessed physical activity level to find that individuals that had participated in the resistance training had adopted a more physically active lifestyle (Perrig-Chiello et al., 1998). The notion of educating individuals on proper exercise parameters in order to increase their comfort level in engaging in physical activity along with exposing people to various types of training to allow them to discover one that they enjoy could be chief contributors to enhancing physical activity levels. Ultimately, if individuals increase the amount of exercise that they participate in, they will be more likely to enhance their fitness levels, resulting in improvements in executive function and overall cognition.

Exercise Intensity and Duration. Exercise intensity, the extent of the physiological disruption or stress of the physical activity being performed, is multifactorial. Age, training status, gender, environmental conditions and health status all affect both the perception of exercise intensity and actual, absolute exercise intensity as measured by energy expenditure or $\mathrm{VO}_{2}$. Intensity can be determined by a number of subjective and objective methods including rating of perceived exertion (RPE), heart rate reserve (HRR), maximal heart rate $\left(\mathrm{HR}_{\max }\right)$, and oxygen uptake $\left(\mathrm{VO}_{2}\right)$. Regardless of how it is determined, intensity can play a role in altering executive function. Reviews conducted on executive function literature fault some of the variation in research outcomes on the prescribed intervention intensity (Angevaren, Vanhees, 
Nooyens, Wendel-Vos, \& Verschuren, 2010). Implications that specifications in intensity of exercise may result in benefits for cognitive function have been suggested (Angevaren et al., 2010; Fabre et al., 2002). Fabre and collogues (2001) utilized individualized intensity to promote aerobic enhancement for each participant resulting in substantial positive improvements in cognitive function. This may endorse the need for personalization in exercise prescription to elicit the most efficient and encompassing results on both fitness level and enhancement of executive function. Energy expenditure of an exercise bout as well as total energy expenditure has been shown to be inversely related to cognitive decline and positively predicted memory task success (Floel et al., 2010; Middleton et al., 2011). Overall, recommendations for moderate to intense exercise have been well supported for improvements in executive function (Erickson et al., 2011; Radak et al., 2010; Stroth et al., 2009; van Uffelen, Chinapaw, Hopman-Rock, \& van, 2009; Zlomanczuk et al., 2006).

Duration of exercise training, or the time spent participating in the exercise bout, is considered to be another integral factor in determining effectiveness of an exercise intervention on cognitive function. Some studies report findings to support that duration provided the greatest impact on cognitive function, specifically in the areas of planning (Davis et al., 2008). A review of literature conducted by Denkinger (2012) indicated that the gold standard for cognitive intervention via exercise is 30 minutes of physical activity performed 5 times per week. This standard corresponds to the recommendations issued by the American College of Sports Medicine (ACSM) for general cardiorespiratory health and wellness. The variation in exercise guidelines diverge with duration of exercise bouts or sessions. While ACSM recommends 30-60 minutes of continuous exercise, Denkinger's (2012) review suggests that exercise sessions should not exceed 30 minutes. There are other studies that report positive 
executive function results with exercise durations lasting 40 to 90 minutes (Davis et al., 2008;

Radak et al., 2001; van Uffelen et al., 2009). Ultimately, it would appear that if the ACSM gold standard for cardiovascular exercise is met cognitive improvements will also be evident.

Many physiologic responses hinge on the intensity level and duration of the exercise being performed. Gene expression, substrate response (norepinephrine), heart rate, and pulmonary function (Carles S Jr et al., 2007). The magnitude of the stress placed on the exercising body will determine the scale of the response elicited by the body. For this reason, duration and intensity need to be among the list of factors to consider when prescribing an exercise intervention. Both duration and intensity have been argued to be the most important components of exercise prescription when concentrating on executive function; however both variables administered at the recommended levels may provide the most abundant gains in executive function (Asl et al., 2008; Ahamed et al., 2007; Angevaren et al., 2010; Budde et al., 2008; Carles S Jr et al., 2007; Coe et al., 2006; DiLorenzo et al., 1999; Fabre et al., 2002). As in any body of literature, contradictions exist, thus there are some studies that will argue that neither duration nor intensity are important moderating factors related to executive function (Smith et al., 2010).

\section{Exercise Environment}

The environment that an individual is immersed in can play a major role in the maintenance and development of executive function (Bielak, 2010; Zec, 1995). An environment that provides the inhabitants a means of stimuli, known as an 'enriched environment', affords the optimal condition for cognitive function. This stimulus could be obtained from exercise, cognitively challenging activities, or a complex environment. While exercise has been discussed in detail, the concept of environmental enrichment has not. A complex environment can be 
described as an individual's exposure to changing contextual variables or diverse stimuli that require the individual to make multiple, complex decisions that place demand on their cognitive skills. Environment can pertain to lifestyle activities, education, as well as occupation and can positivity influence neurologic processes to become more efficient (Bielak, 2010; Hultsch et al., 1999; Zec, 1995). Adaptivity and plasticity of neural structures via cerebral blood flow, increasing numbers of neural synapses or synaptic organization, hippocampal neurogenesis, neuronal survival, or an upsurge in neurochemical availability are all possible physiological explanations for this phenomenon (Bielak, 2010; Blackmore et al., 2009; Galvan \& Jin, 2007; Galvan \& Bredesen, 2007; Hillman et al., 2008). These adaptations are theorized to occur within an enriched environment due to the fluctuating environmental needs resulting in the necessity for the central nervous system to recognize and perceive varying sensory input then determine and create an appropriate motor response corresponding with proper sequencing, timing and coordination necessary for the action (Binder, Storandt, \& Birge, 1999; Hultsch et al., 1999). The key component essential for the complex environment to deliver is a necessity for engagement in cognitively demanding situations (Hultsch et al., 1999). The concept of environmental support has also been addressed in the literature imposing that if older adults are not supported or explicitly instructed to participate in more cognitively demanding activities, they will disengage from more complex cognitive processing events (Park \& Reuter-Lorenz, 2009). Disengagement from more elaborate processes reduces the opportunity for fortification of cognitive flexibility and lends to increased cognitive frailty (Park \& Reuter-Lorenz, 2009). Inclusion of motor or mental complexity within the exercise stimulus can further promote positive cognitive outcomes (Budde et al., 2008). Exercise can utilize environmental stimuli, 
motor complexity, neurobics (mental exercises) or any combination of these activities to assist in further development of an enriched environment during physical activity.

\section{The Aquatic Environment}

The aquatic environment boasts of being one of the most malleable exercise environments available and can provide a variety of benefits to a range of populations. The physical properties of water modify many physiological aspects of human function and thus have a direct reaction on exercise physiology. The response elicited on the individual immersed in water is variable, thus, it is necessary to review the biophysiologic aspects of water.

Density \& Specific Gravity. In an aquatic setting, density of an individual's body in relation to the water's density is very important for maintenance of body position. Density can be defined as mass per unit volume and ultimately determines if a body will float or sink. According to Archimedes principal, the body will displace a volume of water weighing somewhat more than their body resulting in an upward thrust equal to the volume of water displaced (Becker BE \& AJ Cole, 2011). Due to the importance of the relationship between the density of the body displacing the water and the density of the water itself, this ratio is given the term specific gravity. Water has the specific gravity of 1 , while lean body mass is 1.1 and fat mass is 0.9 . Generally speaking, the body will have a specific gravity slightly less than water allowing for a net up-thrust or buoyant effect on a submerged body.

Density and specific gravity can provide new challenges to individuals exercising in the water. Because of individualistic density, specific gravity and body fat distribution, each person entering the water will be confronted with modifying muscular contractions, somatic awareness and concentration to maintain appropriate exercising posture. This can be made easier for some by increasing grounding forces (the effect of gravity) via adding weights to an individual's 
ankles or allowing time for adjustment by working in more shallow water where gravity remains the dominating force over buoyancy. Thus, the effects can be gradually progressed to allot for greater stimulus from the aquatic environment. This can add another dimension to exercise intended to promote executive function. As noted previously, adding coordinative, concentration or other cognitively demanding variables to exercise can result in more positive outcomes on cognitive performance (Bielak, 2010; Binder et al., 1999; Blackmore et al., 2009; Galvan \& Jin, 2007; Galvan \& Bredesen, 2007; Hillman et al., 2008; Small et al., 2006; Stroth et al., 2009; Zec, 1995).

Buoyancy. As an individual enters the water and becomes immersed, water is displaced and a buoyant effect is experienced. As the individual submerges deeper into the water, greater buoyant effects are noted. For instance, if in waist-deep water, the water's buoyancy will offload approximately $50 \%$ of their body weight, while if in neck-depth water roughly $85 \%$ of their body weight will be unloaded (Becker BE \& AJ Cole, 2011). An individual can utilize the effects of buoyancy to completely off-load their body with the assistance of flotation equipment allowing them to move in the water without any gravitational compression forces whatsoever. This feature of the aquatic environment has many advantageous attributes. Firstly, it provides an option for 'weightless' exercise. This can be very beneficial for individuals with joint pain, back pain, pain associated with lower extremities, weight bearing restrictions, chronic pain disorders, or discomfort accompanying overweight or obese status. A reduction or elimination of pain experienced during aquatic exercise has been noted for a variety of physiological issues associated with pain (Evcik, Yigit, Pusak, \& Kavuncu, 2008; Forestier et al., 2010). A second benefit that buoyancy offers is assistance, resistance or support. The entire body or specific exercises can be assisted by buoyancy by moving with the upward force supplied, resisted by 
moving against the upward force, or supported by performing exercises that use the suspension qualities of buoyancy. Support from buoyancy also reduces the risk of falling when balance and strength are challenges (Abbasi, Sadeghi, Tabrizi, Bagheri, \& Ghasemizad, 2012; Hale, Waters, \& Herbison, 2012; Suomi \& Koceja, 2000).

The attributes associated with buoyancy can allow for greater comfort and involvement when participating in exercise. They can also provide a means of progression as well as a tactic to individualize prescribed exercise for group or one-on-one exercise sessions. Buoyancy, in relation to density and specific gravity, also can off-set the body's equilibrium when immersed to provide a challenge for postural maintenance and locomotor control. The additional perturbation can increase the cognitive demands during the exercise intervention to provide for greater cognitive stimulus resulting in improvements in executive function.

Hydrostatic Pressure. Hydrostatic pressure is the pressure exuded upon a body immersed in water. "The water exerts a pressure of $22.4 \mathrm{~mm} \mathrm{Hg} /$ foot of water depth...Thus a human body immersed to a depth of 48 inches is subjected to a force equal to $88.9 \mathrm{~mm} \mathrm{Hg}$, slightly greater than normal diastolic blood pressure" (Becker \& Cole, 2011 pg 24). The applied pressure provides support to the physiological systems by assisting blood return to the heart and lymph redistribution throughout the body (Gulick, 2010; Mourot et al., 2010). By enhancing the mechanisms for fluid distribution, a reduction in edema (swelling) results (Tidhar, Drouin, \& Shimony, 2007; Ambroza \& Geigle, 2010). The effect of hydrostatic pressure enhancing venous return to the heart is a major contributing factor to a net reduction in heart rate while immersed (Becker BE \& AJ Cole, 2011; Kruel, 2009). Veins are highly affected by external pressure, including muscular compression and hydrostatic pressure, thus immersion results in a net increase in central venous pressure leading to a greater return of blood to the heart to shift the 
heart rate downward. The result of this blood shift is an increase in central volume, cardiac volume, mean stroke volume and cardiac output leading to a more efficient cardiovascular system. The efficiency of the CV system necessitates new parameters for intensity-driven exercise in the water. Heart rate reduction is highly variable from person-to-person so the use of an individualized formula such as the Kruel heart rate deduction is necessary for intensity prescriptions during aquatic exercise (Graef \& Kruel, 2006). Another result of the increased blood distribution is an increase in circulatory capacity, or there is greater availability of blood to shunt to the working tissues. Though circulatory results of immersion have not been studied in the brain, it is accepted that muscular blood flow more than doubles with immersion $(1.8$ $\mathrm{ml} / \mathrm{min} / 100 \mathrm{~g}$ of tissue on land to $4.1 \mathrm{ml} / \mathrm{min} / 100 \mathrm{~g}$ of tissue in neck-depth water) as does the metabolic waste removal capability of the circulatory system (increases seen were $225 \%$ above that of land metabolic waste removal) (Balldin, Dahlback, \& Lundgren, 1971). Along with the cardiovascular and fluid redistribution, hydrostatic pressure also supplies enough pressure around the thoracic cavity, via direct pressure and reallocation of blood into the chest cavity, to increase the work of respiration by $60 \%$ (Hong, Cerretelli, Cruz, \& Rahn, 1969). In addition, hydrostatic pressure is theorized to provide a stimulation overload via skin sensory nerve endings including temperature, touch, and pressure receptors throughout the body to elicit a reduction in pain sensation (Gunther V, 1994). All of these varying stimuli produce an environment that provides the body with novel stimuli that may further engage the cognitive processes to reduce cognitive dysfunction. Enhanced circulation may also prove to further enhance cognitive maintenance or development. As stated previously, the literature supports that improvement in cerebral blood flow may produce positive structural modifications in cerebral tissue that promote 
executive function (Barnes et al., 2003; Bielak, 2010; Deeny et al., 2008; Lachman ME, 2006; Man et al., 2010; Scherder et al., 2010; Small et al., 2006).

Viscosity. The viscosity, or relative thickness of a liquid, delivers a much higher resistance than that of air resulting in an increased amount of force required to elicit movement. Viscosity of water does not become a relative variable until movement is produced by a body or limb. The force needed to move a body, limb or object through the water is highly related to the speed of movement being produced, the frontal surface area and shape of the object being moved through the water and the amount of turbulent flow present in the water during the movement. Because the aquatic environment is all-encompassing when a body is submerged, any movement that is made while submerged is resisted. This is very different from movements that are made on land where gravity is the only resistive force applied. When a movement is performed on land, there are two contractions that occur at the facilitating muscle groups: a concentric contraction and an eccentric contraction. The concentric, or shortening contraction transpires during a resistive movement (movement opposite of gravity or against gravity) while eccentric, or lengthening contractions occur as the same muscle has to control the movement in the direction of gravity. In the water, this drastically changes. Because of the resistance from viscosity, movements performed without incorporation of equipment elicit only concentric contractions and these contractions take place with the muscle pair. So instead of only working a single muscle or muscle group, you are able to train the muscle pair effectively with a single exercise. This statement is only true of exercises performed without the use of equipment; muscular contractions in the water change when equipment is introduced (see table 2.3 for further clarification of muscle contractions). 
Table 2.3: Contraction Variations Occurring in an Aquatic Environment

\begin{tabular}{|c|c|c|}
\hline Environment \& Equipment & Flexion of the elbow & Extension of the elbow \\
\hline Land Movement & Concentric Biceps & Eccentric Biceps \\
\hline $\begin{array}{l}\text { Submerged with NO } \\
\text { Equipment }\end{array}$ & Concentric Biceps & Concentric Triceps \\
\hline $\begin{array}{l}\text { Submerged with Drag } \\
\text { Equipment }\end{array}$ & Concentric Biceps & Concentric Triceps \\
\hline $\begin{array}{l}\text { Submerged with Buoyant } \\
\text { Equipment }\end{array}$ & Eccentric Triceps & Concentric Triceps \\
\hline $\begin{array}{l}\text { Submerged with Weighted } \\
\text { Equipment }\end{array}$ & Concentric Biceps & Eccentric Biceps \\
\hline $\begin{array}{l}\text { Submerged with Rubberized } \\
\text { Equipment }\end{array}$ & $\begin{array}{l}\text { Rubberized band anchored on } \\
\text { ground: Concentric Biceps } \\
\text { Rubberized band anchored } \\
\text { overhead: Eccentric Triceps }\end{array}$ & $\begin{array}{l}\text { Rubberized band anchored } \\
\text { on ground: Eccentric } \\
\text { Biceps } \\
\text { Rubberized band anchored } \\
\frac{\text { overhead: Concentric }}{\text { Triceps }}\end{array}$ \\
\hline
\end{tabular}

Due to the variability of contractile forces, muscular recruitment and stimuli, neuromuscular recruitment patterns are highly altered in an aquatic setting (Colado, Triplett, Tella, Saucedo, \& Abellan, 2009; Colado, Tella, Triplett, \& Gonzalez, 2009; Colado, Tella, \& Triplett, 2008; Triplett et al., 2009). Theoretically, this could yield adaptation to cerebral function, hormonal interactions or even neurogenesis by adding new, complex movement patterns into an individual's exercise prescription to develop a more enriched environment (Budde et al., 2008; Hultsch et al., 1999; Klusmann et al., 2010; Man et al., 2010; Stroth et al., 2009). It has also been noted that combined exercise training (resistance training paired with aerobic training) may improve to a greater degree than just utilizing a single modality (Colcombe 
\& Kramer, 2003). The aquatic environment lends well to this theory as every movement produced is counteracted by the viscous force of water thus promoting resistance training while performing aerobic training.

Conclusions on the Aquatic Environment. The greater the level of immersion, the greater the effect the aquatic environment will elicit on the immersed body. Many physiological effects are produced during immersion that could potentially affect executive function. The environment in and of itself, affords various new stimuli to further develop the exercise session from mere exercise to exercise within an enriched environment. The challenges that the physical attributes of the aquatic environment lend to individuals in the pool also drive the requirement for added concentration, variance of muscular contractions and coordination while performing aquatic exercise. The buoyant support that the water provides may increase comfort during exercise for a variety of populations allowing them to enjoy the exercise experience more while promoting participation longevity. Lastly, the increase in circulatory function that augments blood availability may potentially lead to increased cerebral circulation which has been definitively seen to promote neural structure maintenance and growth (Barnes et al., 2003; Bielak, 2010; Deeny et al., 2008; Lachman ME, 2006; Man et al., 2010; Scherder et al., 2010; Small et al., 2006).

All of these aspects of participating in aquatic exercise have the potential to promote, enhance or even improve upon the effects of dry-land exercise on cognitive function. It is well accepted that land exercise promotes a multifaceted chain of reactions to stimulate positive alterations in executive function but these progressive results could be augmented by modifying the exercise medium. With the addition of immersing the body while performing exercise, the individual could potentially experience a heightened level of executive function adaptation 
prompted by greater environmental stimulation and improved circulation. In addition, adherence and continuation of exercise participation may also be superior. Studies support a higher level of both adherence and exercise continuation when the physical activity is performed in water (Bennell \& Hinman, 2011; Munguia-Izquierdo \& Legaz-Arrese, 2008; Kang, Ferrans, Kim, Kim, \& Lee, 2007; Wang, Belza, Elaine, Whitney, \& Bennett, 2007; Belza et al., 2002). This may be, in part, due to the sensation of security that the water provides, the reduction in pain stimulated by hydrostatic pressure, or the off-loading of the joints promoted by the upward force of buoyancy. Regardless of the reasoning behind this fact, continuation of exercise is noted to be paramount for continued cognitive benefits. Thus the water may be the ideal medium for this sole reason.

\section{Exercise Implications for Memory Disorders}

Memory impairment disorders, including Alzheimer 's disease (AD), various types of dementia, mild cognitive impairment (MCI), and vascular- or degeneration-related cognitive dysfunctions, are characterized by difficulty or inability to successfully navigate the domains of executive function, language, motor activity and object recognition (Center for Disease Control, 2012). Dementia predominately affects individuals over the age of 60 and the risk for developing one of these diseases, especially $\mathrm{AD}$, rises with progressing age. The average life expectancy has steadily increased with every passing year since 1980 (70.0 years old in 1980 to 80.9 years old in 2009) due to the tremendous advances in medicine and medical technologies along with social and environmental conditions (National Center for Health Statistics, 2012). The prevalence of longevity and the incidence of memory impairment disorders show a parallel relationship, with the risk of developing a memory disorder, such as $\mathrm{AD}$, doubling every five years beginning at age 65(Center for Disease Control, 2012). "Alzheimer's disease is the sixth 
leading cause of death in the United States and the fifth leading cause of death among persons age 65 years and older" (Hebert LE, 2003). In addition to its astonishing prevalence, AD alone is assessed to cost 183 billion dollars annually and that healthcare burden does not include the other variations of cognitive impairment (Alzheimer's Association, 2011). With the prevalence of cognitive impairment disorders on the rise and the number of healthcare dollars devoted to treatment, the necessity for prevention, treatment and symptom retardation is paramount.

The risk factors for memory disorders are multidimensional and cannot all be reduced with lifestyle modifications such as exercise. However, a large proportion of the recognized risk factors, such as CVD, Type 2 diabetes, HTN, elevated lipid levels, obesity, and inflammation, can be lessened by including regular, moderate to intense physical activity within one's lifestyle. In fact, a sedentary lifestyle is one of the most prominent risk factors attributed to developing dementia (Radak et al., 2010). This is credited to the ability of cardiovascular exercise to physiologically moderate cerebral tissue atrophy, improve vascularization via angiogenesis, and escalate neurogenesis, increase cerebral circulation, and increase differentiation of neuron structures (Bates et al., 2009a; Bielak, 2010; Burns et al., 2008; Erickson et al., 2009; Hultsch et al., 1999; Myers, 2008; Scherder et al., 2010; Small et al., 2006; Van Boxtel et al., 1997). Exercise is also recognized as a means of providing environmental stimuli that can enhance executive function in both diseased and normal populations (Bielak, 2010; Binder et al., 1999; Blackmore et al., 2009; Galvan \& Jin, 2007; Hultsch et al., 1999; Small et al., 2006; Stroth et al., 2009; Zec, 1995). There have also been strong links associated with obesity, CVD and other vascular-related diseases for increasing risk for developing AD (Bates et al., 2009a; Bates et al., 2009b; Small et al., 1997) and it is an accepted fact that exercise reduces the risk of developing these, along with a variety of other risk factors associated with cognitive impairment (Chodzko- 
Zajko \& DN Proctor, 2009). These factors provide evidence suggesting that incorporating regular exercise into activities of daily living may modify the process of dementia and other cognitively related diseases (Burns et al., 2008; Small et al., 2006).

The act of participating in exercise as well as accumulating fitness gains by involvement in regular physical activity have been noted to have positive effects on risk reduction as well as symptom delay associated with memory disorders (Angevaren et al., 2008; Burns et al., 2008; Birren \& Fisher, 1995; Budde et al., 2008; Carles S Jr et al., 2007; Clarkson-Smith \& Hartley, 1990; Coles \& Tomporowski, 2008; Floel et al., 2010; Goekint et al., 2010; Hillman et al., 2008; Komulainen et al., 2008; Komulainen, 2010; Logsdon, McCurry, Pike, \& Teri, 2009; McAuley et al., 2011; Pontifex et al., 2009; Swardfager et al., 2010; Voss et al., 2011). Burns and colleagues (2009) study on Cardiorespiratory fitness and Brain atrophy in early Alzheimer's Disease, demonstrated that higher fitness levels in early $\mathrm{AD}$ were related to cerebral volume preservation. It is also remarkable that these results were independent of age and the severity of dementia (Burns et al., 2008). Other studies support these results and extend the findings to include the notion that populations at a higher risk for developing cognitive impairments tend to exhibit the greatest protective effects manifested from participation in exercise (Deeny et al., 2008). The cognitively shielding effects that exercise provides can be reaped prior to cognitive impairment development as a preventative strategy, during the early stages of the disease as a means to slow down the effects, or even in the late stages of the pathology, as a means of slowing or improving cognitive function (Nichol et al., 2007). In fact, the threshold of training necessary for promotion of cognitive improvements may be lower for individuals that have a present cognitive pathology than in normal, age-related cognitive decline (Nichol et al., 2007). 
Participation in exercise early in life may also provide protective cognitive effects throughout the ageing process. Studies have established a positive relation between being physical active during ages 15-60 years and reduction of developing cognitive impairments such as dementia (Denkinger et al., 2012; Friedland et al., 2001; Dik et al., 2003). Interestingly, the benefits experienced from past involvement in exercise were enhanced by currently participating in physical activity (Richards, Hardy, \& Wadsworth, 2003). Though the importance of exercising throughout the lifespan is evident, current activity levels show the greatest effects on executive function (Wilson et al., 2005). Abbott and colleagues (2004) concluded that men that walked at least 2 miles a day reduced their risk for developing dementia 1.8 times compared to those who walked the least. These findings promote the inclusion of exercise throughout the lifespan and make evident the concept that it is never too late to begin exercise for cognitive protection and improvement (Denkinger et al., 2012; Liu-Ambrose et al., 2010).

Many of the physiological changes observed in the brain that have been discussed occur at a more rapid rate in individuals with cognitive diseases, including: frontal lobe dysfunction and volume reductions in grey matter, white matter, hippocampus, dentate gyrus, and cerebellum as well as reductions in BDNF and IGF-1(Burns et al., 2008; Cabeza et al., 2002; Erickson et al., 2009; Erickson et al., 2011; Kim et al., 2010; Park \& Reuter-Lorenz, 2009; Peters, 2006; Smith et al., 2010; Van der Borght et al., 2007; van Praag, 2009; Voss et al., 2011). As noted previously, these clinical manifestations of cognitive impairment can be reduced or even halted with the inclusion of exercise making physical activity paramount for those wanting to maintain cognitive function throughout their lifetime (Asl et al., 2008; Barnes et al., 2007; Birren \& Fisher, 1995; Budde et al., 2008; Burns et al., 2008; Blackmore et al., 2009; Cabeza et al., 2002; Chaddock et al., 2011; Davis et al., 2008; Erickson et al., 2009; Erickson et al., 2011; Kim et al., 
2010; Kramer \& Erickson, 2007; Lautenschlager et al., 2008; McAuley et al., 2011; Myers, 2008; Park \& Reuter-Lorenz, 2009; Pereira et al., 2007; Peters, 2006; Van der Borght et al., 2007; van Praag, 2009; Voss et al., 2011; Swardfager et al., 2010; Zlomanczuk et al., 2006; Yu et al., 2009).

Though participation in physical activity and accruing physical fitness is imperative for cognitive maintenance, there may be obstacles present for taking part in these activities. Fitness declines related to behavioral changes observed in individuals with cognitive impairments may contribute to an increase in mental demise (Burns et al., 2008; Denkinger et al., 2012). This may also be a generalizable factor for the aging population. With increasing age, individuals become more reluctant to begin an exercise program (particularly if they do not have a history of participation in exercise), do not have an understanding about appropriate forms of exercise or exercise techniques, possess a fear of injuring themselves or experiencing pain, or lack positive health status or social support structure (Logsdon et al., 2009). These variables make the inclusion of physical activity even more challenging for this population, thus introducing a fitness program that reduces these apprehensions is vital. The aquatic environment can provide an atmosphere that reduces risk of injury and pain, provides physiological support for those with less than optimal health status, and, with the inclusion of a group fitness class with a qualified instructor, can provide social support and exercise education (Becker BE \& AJ Cole, 2011; Belza et al., 2002).

\section{Theoretical Framework for Exercise and Cognitive Decline}

Multiple theories exist on the reasoning behind age related cognitive decline. These theories represent a broad knowledge base developed from the current literature and shaped into a theoretical framework to guide future research and provide hypotheses for both decline and 
gain related to aging and executive function. There are four widely accepted theories associated with age related cognitive decay: executive-control, speed, controlled-processing and the visuospatial hypothesis (Colcombe \& Kramer, 2003; Park \& Reuter-Lorenz, 2009). Though these theories may be referred to by various names, the content for each hypothesis is the same across the spectrum. The speed hypothesis refers to the concept that perceptual speed is a necessity for cognitive function and the age-related decline experienced can be attributed to a reduction in ability to process information with speed (Colcombe \& Kramer, 2003; Park \& Reuter-Lorenz, 2009). Controlled-processing is the notion of delineating between effortful versus automatic memory. This theory proposes that as individuals age, their ability to utilize explicit memory is highly reduced while their ability to employ memory for gist or familiar stimuli remain relatively good (Colcombe \& Kramer, 2003; Park \& Reuter-Lorenz, 2009). The visuospatial theory denotes that sensory deficits and deterioration are interconnected to decline in memory within the ageing population. Both visual and auditory inputs have been noted to serve as "powerful regulators of intellectual performance in old and very old age" (Baltes \& Lindenberger, 1997). This has been primarily attributed to age-induced dedifferentiation or the decline in neural specialization seen in the aging brain (Colcombe \& Kramer, 2003; Park \& Reuter-Lorenz, 2009). Lastly, the executive-control hypothesis denotes a decline in cerebral coordination, inhibitory functions, working memory, planning, and scheduling that result in general attentional disregulation, information manipulation and an overall reduction in executive function (Colcombe \& Kramer, 2003; Park \& Reuter-Lorenz, 2009). Gains in fitness have been proposed to assist in positively altering factors in many of these theoretical frameworks (Colcombe \& Kramer, 2003; Park \& Reuter-Lorenz, 2009). 
Park \& Reuter-Lorenz (2009) further addressed and organized the current theoretical framework with the publication of their "Scaffolding Theory of Aging and Cognition" or STAC. STAC stems from the idea that despite all of the physiological maladaptation that occurs during the aging process, older adults remain highly functional within the cognitive arena. Within the confines of STAC, maintenance is noted to be due to the ever-changing and adaptive compensatory scaffolding constructed within the neural network that allows for variation in recruitment pattern for task achievement (Park \& Reuter-Lorenz, 2009). In other words, if the desired task requires a neural pathway that is dysfunctional, instead of disengaging from the task, new cerebral pathways are created to portray the same or a similar message so that the task can be completed. This is the idea that fits the basic concept of learning. When presented with a challenge, the brain responds by assessing the requested needs for the accomplishment of a task and delineating a neural construct to meet those needs resulting in creation of new neural pathways. Another component of this theory suggests that old neural pathways may also be resurrected (Park \& Reuter-Lorenz, 2009). When addressing neural imaging while acquiring a new skill, it is evident that dedifferentiation is prevalent until the skill is learned (Davis et al., 2007). As the individual becomes more proficient at the skill, the neural recruitment pattern becomes more specialized enabling a quicker response and outcome. STAC proposes that as individual's age and neural degeneration occurs, the brain can 'remember' and regress to the dedifferentiated means of relaying information in order to meet the task demands (Park \& Reuter-Lorenz, 2009). Thus "scaffolding can be understood as circuits that provide supplementary, complementary, and, in some cases, alternative ways to achieve a particular behavioral output or cognitive goal" (Park \& Reuter-Lorenz, 2009, pg 185). 
The prefrontal cortex is noted to be the single most adaptive portion of the brain and, as noted previously, is highly affected by the aging process. Because of its adaptability, this portion of the brain is noted to be the primary site for scaffolding formation (Park \& Reuter-Lorenz, 2009). This theory is supported by neural imaging that reveals greater activation levels in the prefrontal lobes within the aging brain (Davis et al., 2007). Fortunately, the prefrontal cortex is also one of the primary sites for enhancement from participation in physical activity (Birren \& Fisher, 1995; Cabeza et al., 2002; Myers, 2008; Park \& Reuter-Lorenz, 2009; Peters, 2006; Geda et al., 2010; Voss et al., 2011). The frontal lobe as well as white and grey matter within that lobe are spared or even created with the inclusion of regular physical activity allowing for greater adaptation, or scaffolding formation (Birren \& Fisher, 1995; Cabeza et al., 2002; Myers, 2008; Park \& Reuter-Lorenz, 2009; Peters, 2006; Geda et al., 2010; Voss et al., 2011). Ultimately, all of the positive neuro-physiological outcomes observed with exercise and fitness improvement can be related to the ability to build compensatory scaffolding with greater proficiency resulting in enhanced executive function. These effects are further enhanced by the utilization of cerebral training and repetition which can be provided with a more complex exercise program paired with an enhanced environment (Park \& Reuter-Lorenz, 2009). These are factors that aquatic exercise and the aquatic environment can easily provide.

\section{Conclusion}

The aging process has vast effects on executive function that result in diminished quality of life, increased medical costs and loss of independence. Age related changes in brain structure primarily occurring within the frontal, parietal and temporal lobes beginning after the age of 30 , have been distinguished as one of the primary causes related to age related cognitive decline (Raz \& Rodrigue, 2006; Park \& Reuter-Lorenz, 2009; Leal \& Yassa, 2013). Further decrements 
can be attributed to a variety of cognitive risk factors including cardiovascular disease, hypertension, lipid profile, type 2 diabetes, and inflammation (Stampfer, 2006). These risk factors have been noted to work against cognitive function promoting a more rapid decline in many cognitive abilities. Fortunately, these neuro-physiological alterations and cognitive risk factors can be curtailed or even reversed with the inclusion of physical activity and accrual of fitness. Exercise can acutely or chronically affect the somatic systems to provide alterations resulting in improvements in executive function. Both aerobic and anaerobic exercise training have been purported to assist in positive cognitive change. A compelling argument has been made that combining these two exercise modalities achieves an even higher level of cognitive improvement.

The aquatic environment can provide a comfortable setting for exercise to take place while enhancing the simplest of aerobic exercises to include a resistance component. The varying physical properties of water can create assistance, resistance and support with movement while providing a stimulus that could be recognized as environmental enrichment. The basic physiologic principals of water in conjunction with the wide array of physical risks and fears associated with diminished executive function or the aging population in general, makes the aquatic environment ideal for exercise facilitation. Both the physiological attributes (somatic stimulation and enhanced circulation) as well as the environmental qualities (support, resistance, and comfort) of exercising in the water could arguably portray aquatic exercise as the ideal mode and medium to enhance cognitive function.

\section{Summary of Literature in Accordance with Research Questions}

Research Question One asks: Does participation in a 10-week aquatic exercise class result in a statistically significant ( $p \leq .05$ ) improvement in cognitive function when compared to the control group? 
Aerobic training has been noted to have both acute and chronic effects on various components of executive function (Liu-Ambrose et al., 2010; Baker et al., 2010; Barnes et al., 2003; Bielak, 2010; Budde et al., 2008; Davis et al., 2007; Davis et al., 2007; Davis et al., 2008; Liu-Ambrose et al., 2008; Swardfager et al., 2010; Teixeira et al., 2012; Hogan et al., 2013; Joyce et al., 2014). Research supports the link between participation in chronic aerobic exercise and: improvements in spatial learning, memory, cognitive capacity, selective and divided attention, working memory, cognitive flexibility, planning, inhibition, decision making, problem solving, cognitive speed, and overall cognitive function (Angevaren et al., 2008; Baker et al., 2010; Barnes et al., 2003; Birren \& Fisher, 1995; Budde et al., 2008; Fabre et al., 2002; Hillman et al., 2009; Hillman et al., 2008; Hillman et al., 2003; Ke et al., 2011; Liu-Ambrose et al., 2010; Man et al., 2010; Scherder et al., 2010; Smith et al., 2010; Van der Borght et al., 2007; Teixeira et al., 2012; Yaguez et al., 2011). Aerobic training has also been correlated with increased cerebral electrical activity and neurotransmitter secretion as well as reductions in neural apoptosis, increased growth factor modulation, increased vascularization, declines in brain volume loss, and memory loss (Asl et al., 2008; Baker et al., 2010; Berkman et al., 1993; Blumenthal \& Madden, 1988; Burns et al., 2008; Chaddock et al., 2010; Chan et al., 2005; Clarkson-Smith \& Hartley, 1989; Deeny et al., 2008; Erickson et al., 2011; Erickson et al., 2009; Fabre et al., 2002; Floel et al., 2010; Goekint et al., 2010; Hillman et al., 2009; Hillman et al., 2008; Ke et al., 2011; Kim et al., 2010; Klusmann et al., 2010; Komulainen, 2010; Komulainen et al., 2008; McAuley et al., 2011; Nation et al., 2011). The overall outcome of exercise is enhanced immune condition of the brain and its structures that results in improved neural plasticity. 
The body systems related to cognition are acutely affected by a single bout of exercise and exhibit chronic effects as a result of continuous exercise training. Primary alterations credited to participation in exercise exhibited in the body correlating to improvements in executive function include increased cerebral circulation and increased vascularization (Asl et al., 2008; Erickson et al., 2011; Goekint et al., 2010; Kim et al., 2010; Kramer \& Erickson, 2007; Komulainen et al., 2008; Lachman ME, 2006; Liu-Ambrose et al., 2008; Radak et al., 2010; Smith et al., 2010; Voss et al., 2011). These primary exercise-induced adaptations are recognized to result in deposition of new neuronal structures as well as differentiation of those structures within targeted areas of the brain ultimately leading to improved cognitive performance and executive function.

In addition, modifiable risk factors and medical conditions have been linked to cognitive deterioration, these include: diabetes, smoking, cardiovascular disease, hypertension (high blood pressure), cerebrovascular disease, depression, hypercholesterolemia (elevated cholesterol), sedentary lifestyle, and irregular participation in mentally or socially stimulating activities (Strength of the relation between the risk factor and cognitive impairment is depicted in Table 2.1) (Baker et al., 2010; Barnes et al., 2003; Myers, 2008; Small et al., 2006; Chuang et al., 2013). All of these factors have been noted to be positively impacted by chronic physical activity. Though there are conflicting findings for many of these risk factors, there is a strong body of evidence supporting the belief that these factors, independently or cumulatively work against cognitive function.

Lastly, the environment that an individual is immersed in can play a major role in the maintenance and development of executive function (Bielak, 2010; Zec, 1995). An environment that provides the inhabitants a means of stimuli, known as an 'enriched environment', affords the 
optimal condition for cognitive function. This stimulus could be obtained from exercise, cognitively challenging activities, or a complex environment. Many physiological effects are produced during immersion that could potentially affect executive function. The environment in and of itself, affords various new stimuli to further develop the exercise session from mere exercise to exercise within an enriched environment. The challenges that the physical attributes of the aquatic environment lend to individuals in the pool also drive the requirement for added concentration, variance of muscular contractions and coordination while performing aquatic exercise.

Research Question Two and Three ask: Does participation in a 10-week aquatic exercise class result in a statistically significant ( $p \leq .05$ ) improvement in cardiovascular fitness when utilizing the Kruel target heart rate deduction to configure individualized target heart rate zones when compared to the control group? Do statistically significant ( $p \leq .05)$ positive changes occur for general fitness and wellness parameters after participation in a 10-week aquatic exercise class when compared to the control group?

Physical Activity and Public Health in Older Adults: Recommendation from the American College of Sports Medicine and the American Heart Association, a policy published by the Centers for Disease Control and Prevention (CDC), recommends 150 minutes of moderate intensity (3.0-6.0 METs) aerobic activity or 75 minutes of vigorous activity ( $\geq 6.0$ METs) per week (Nelson et al., 2007). It is estimated that only 9-26\% of older adults are meeting these recommendations in the U.S. (Tucker et al., 2011) with over 50\% proclaiming no intention to begin an exercise program (Dishman \& Ickes, 1981). It is also evident that physical activity progressively decreases with increasing age (Schutzer \& Graves, 2004). The vast majority of older adults have a tendency to allocate the greater part of their time to sedentary behaviors (behaviors $\leq 1.5$ METs) resulting in atrophy of the body and brain (Evenson et al., 2012; Dogra \& Stathokostas, 2012). 
The aquatic environment boasts of being one of the most malleable exercise environments available and can provide a variety of benefits to a range of populations. The physical properties of water modify many physiological aspects of human function and thus have a direct reaction on exercise physiology. Principals such as buoyancy, hydrostatic pressure, and viscosity alter how the body physiologically reacts and moves while participating in aquatic exercise. The greater the level of immersion, the greater the effect the aquatic environment will elicit on the immersed body. For this reason considerations for exercise training must be taken into account. One such consideration is target heart rate (THR). The trusted Karvonan formula to configure THR is not as easily applicable to the aquatic environment due to the effects of immersion. Thus, the Kruel THR reduction model is applied.

Though alterations should be accounted for when working with participants in the water, past research promotes that cardiovascular fitness and wellness parameters can be improved upon with the inclusion of water aerobics (Honda \& Kamioka, 2012; Kamioka et al., 2010; Kamioka et al., 2011; Rica et al., 2013; Becker BE \& AJ Cole, 2011; Meredith-Jones, Waters, Legge, \& Jones, 2011). Improvements have been noted to occur in relation to cardiovascular fitness, balance, range of motion, strength, power, coordination, and muscular endurance (Honda \& Kamioka, 2012; Kamioka et al., 2010; Kamioka et al., 2011; Rica et al., 2013; Becker BE \& AJ Cole, 2011; Meredith-Jones et al., 2011).

Research Question Four asks: Does participation in a 10-week aquatic exercise class result in a statistically significant ( $p \leq .05$ ) alteration of social support, self-efficacy, motivators, or exercise barriers when compared to the control group?

There are a variety of barriers for older adults associated with participation in physical activity. Health, environment, education, and exercise history are the most commonly cited reasons that older adults do not participate in exercise (Schutzer \& Graves, 2004). Physical 
impediments and limitations can highly affect activity tolerance and adherence while issues with strength, endurance, balance, or cardiovascular efficiency can have a direct impact on an older adult's choice and ability to participate in physical activity. Poor health, including pain and illness, are noted as the primary contributor to sedentary behavior in the elderly (Booth et al., 1997; Cohen-Mansfield, 2003; Schutzer \& Graves, 2004). These physical factors are often augmented by a lack of knowledge on the benefits, appropriate modes and durations, proper execution, and availability of exercise (O'Neill \& Reid, 1991; Booth et al., 1997; Burton et al., 1999; Schutzer \& Graves, 2004). Collectively, these barriers make persuading the elderly to include and maintain physical activity within their daily lives a daunting task.

Motivation is the culmination of intrinsic and extrinsic factors that act on or within an individual to initiate or maintain behavior (Phillips et al., 2004). These factors are both individual and diverse among all populations, including the elderly. Motivating older adults to begin and adhere to regular physical activity is a challenging, yet critical element to program initiation and success (Phillips et al., 2004; SJH Biddle et al., 2012).

There is an interesting relation between exercise barriers and exercise motivators in the aging population. Many identified barriers have also been recognized as motivators to participate in physical activity (Cohen-Mansfield et al., 2006). Issues including deteriorating health, increased time availability, being educated or referred by a physician to exercise, and closer proximity to exercise facilities play the role of both barrier and motivator in the elderly (Cohen-Mansfield et al., 2006; Schutzer \& Graves, 2004; Schutzer \& Graves, 2004). This dichotic relation illustrates the complexity of necessary intervention strategies in this population while providing potential for persuasion to participate in physical activity. 
Self-efficacy, or the individual's belief that they will be able to perform a specified act or behavior, is consistently identified as playing a vital role in adherence to physical activity (Bauman et al., 2012). This principal is a central factor for Bandura's social cognitive theory proposing that environment, personal factors, and behavior interact to produce a level of belief in individual ability (Bandura, 2001; Bandura, 2004). In this construct, the idea of the individual's self-efficacy in relation to their perceived incentive must result in a positive, valued outcome or consequence (Bandura, 2004). Both short-term and long-term outcomes are often sought as incentives for participation. In relation to physical activity, outcomes may include a feeling of accomplishment, enhanced energy levels, or improvements in health or physical status (cardiovascular endurance, muscular strength, muscular endurance, body composition, and flexibility). Though these benefits are likely to transpire with the addition of exercise, initial and continued participation hinges on an individual's self-efficacy relative to being able to complete the task (Bandura, 2004). Assurance that the individual's self-efficacy will promote participation in exercise could be assisted by including educational components (i.e. clear instructions), skill development, and a model of attainable behavior (Seefeldt et al., 2002).

Self-efficacy has been viewed as an important psychosocial mediator at any age, including within the elderly. Improvements in self-efficacy have been shown to increase participation and adherence in physical activity (Brassington et al., 2002) particularly when paired with achievable goals that are gradually advanced and encouraged (Marks et al., 2005). Activities that are not easily achievable may not be appropriate for enhancing or maintaining self-efficacy related to exercise for the novice, or cognitively impaired exerciser. Finding a balance between providing achievable goals and ample challenge may afford the perfect paradigm for exercise programs targeting the aging population. 
Social support is comprised of many varieties of supplied support including: instrumental, informational, emotional, or appraising (Israel BA \& Schurman SJ, 1990; Zimet GD et al., 1988). Within the realm of exercise, these components of social support may play the role of transporting an individual to an exercise class (instrumental), educating someone about the importance of exercise (informational), checking in on how an individual is doing in a newly started program (emotional), or providing feedback or reinforcement during skill allocation (appraising). Any one of these social support components may be provided by family, friends, exercise instructors, or physicians (SJH Biddle et al., 2012; Seefeldt et al., 2002). The level of importance for each of these constituents is going to vary individually, but social support as a whole has been consistently shown to be associated with advancements in activity levels (Bauman et al., 2012).

Social support in the aging population has been reported to increase exercise frequency, duration, and adherence (Walsh et al., 2001; Resnick et al., 2002; Rhodes et al., 2001; Kassavou et al., 2013). Though social support may be an important psychosocial mediator when encouraging exercise success, the availability of social support seems to decrease with age (Resnick et al., 2002; McAuley et al., 2003). Creating outlets for social support within a group exercise environment may be a promising concept for integration of this psychosocial correlate of exercise.

Research Question Five asks: Does the infusion of adult education theory into a 10-week aquatic exercise class assist in behavior change?

According to the literature, there is a necessity for exercise interventions to take a multifaceted approach when working with the ageing adult (Bauman et al., 2012; Sallis JF et al., 2008; SJH Biddle et al., 2012; SJH Biddle et al., 2012). The inclusion of an educational component has become an important constituent within the integrated approach to physical 
activity programming for the aging adult (Schutzer \& Graves, 2004; O'Neill \& Reid, 1991). It serves the role of enlightening individuals on the importance, benefits, methods, and by what means to participate in physical activity. Though education about exercise has been, and should be, used as a tool to promote the inclusion of physical activity into the lifestyles of any population, it seems to be of particular importance within those of advancing age (Schutzer \& Graves, 2004; O'Neill \& Reid, 1991).

Adult educational strategies are very different from approaches utilized to teach children. This is an important fact to recognize in order to have the greatest effect on the individuals being targeted to address lifestyle changes. In order to gain the attention and interest of those that need to be educated on the importance of physical activity, a positive learning climate must be developed (Sullivan R.L. et al., 1990). This is true of any educational moment that may present itself to motivate individuals to begin exercise as well as the continued education that should be provided during exercise. Establishing a positive environment for individuals to feel comfortable learning, making mistakes, and asking questions throughout the acclimation process of exercise could be imperative to their continuation of physical activity as a whole. The process of providing education on the 'why' of exercise may also extend the individuals inclinations to exercise if the reasoning aligns with the adults needs and wants (Lorge, 1947). Providing opportunities for the aging adult to select their own experience via providing options for physical activity integration may also be assistive in the acclimation and learning process. Many adult learning theorist believe that adult learning is rooted in a wealth of past experiences, selfdirection, personal needs and interests, usefulness of the information or skill, and the amount of onus they can link to the experience (Knowles M.S. et al., 2011). If these components can be 
built-in to the exercise experience, there may be a greater effort and adherence response leading to superior outcomes.

Research Question Six asks: Does aquatic exercise provide participants with greater accessibility to exercise participation?

As previously discussed, physical barriers to participating in exercise are one of the most noted reasons elderly individuals do not exercise (Booth et al., 1997; Cohen-Mansfield, 2003; Schutzer \& Graves, 2004). Exercising in the water can provide the aging population with a valuable alternative to seemingly inaccessible land exercise. The physical properties of water can be utilized and manipulated to offer support, assistance, and resistance depending on the needs of the individual. The aquatic environment can provide an atmosphere that reduces risk of injury and pain, delivers physiological support for those with less than optimal health status, and, with the inclusion of a group fitness class with a qualified instructor, can provide social support and exercise education (Becker BE \& AJ Cole, 2011; Belza et al., 2002). 


\section{Chapter Three Methodology}

\section{Review of Purpose}

The overall purpose of this study was to investigate the relation between aquatic-based exercise and executive function in older adults. This investigation was further reinforced by the inclusion of support questions concerning psychosocial mediators, exercise accessibility, adult education theory, physical activity level, and measurement of change in fitness and wellness parameters. Both quantitative and qualitative methods were employed to further enrich the meaningfulness of the data collected.

\section{Research Design}

The present study employed a mixed method design including both qualitative and quantitative data collection and analysis techniques. This mixed method approach increased the probability of improving the quality of the final results and assisted in providing a more comprehensive understanding of the participants views and study outcomes. The quantitative portion was an experimental pretest-posttest design, or a one-variable (i.e., aquatic exercise) multiple-condition (i.e., psychosocial mediators, exercise attitudes and accessibility, and measurement of change in fitness and wellness parameters) design. The qualitative portion of the study was a phenomenological design that sought to capture the experience of the participants as they interacted with the new phenomenon (i.e., aquatic exercise)(Patton, 2002). The overall design of the study in its entirety is diagrammed in Figure 3.1. 


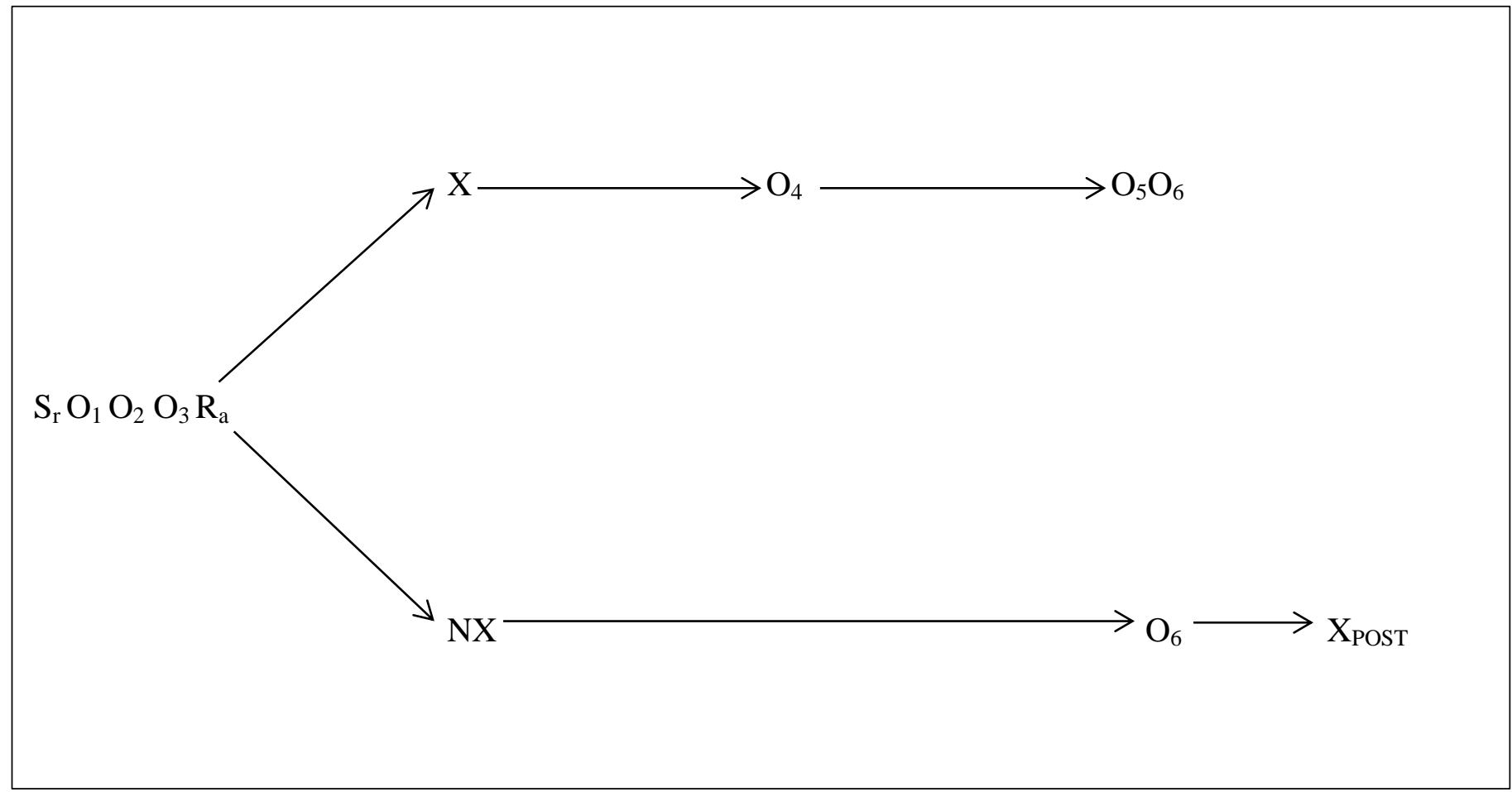

Key to Symbols:

$\mathrm{S}_{\mathrm{r}} \quad=\quad$ Subject Recruitment (volunteers) for the study

$\mathrm{O}_{1}=$ Familiarization to CANTAB

Demographic Data

Past year total physical activity questionnaire

$\mathrm{O}_{2}=\quad \begin{aligned} & \text { Health History } \\ & \text { Pre-Intervention Executive function measurements (CANTAB) }\end{aligned}$

Pre Anthropometric Measurements

$\mathrm{O}_{3}=$ Pre-Intervention Walk Test

Pre-Intervention Psychosocial Mediator Surveys

Pre-Intervention Resting Heart Rate

$\mathrm{R}_{\mathrm{a}}=$ Random assignment of subjects

$\mathrm{X}=10$-Week Aquatic Exercise Intervention

$\mathrm{NX}=\quad$ No Treatment

$\mathrm{O}_{4}=$ Mid-Intervention Psychosocial Mediator Surveys

$\mathrm{O}_{5}=$ Focus Group

$\mathrm{O}_{6}=$ Post Anthropometric Measurements

Changes in Health History

Post-Intervention Executive function measurements (CANTAB)

Post-Intervention Walk Test

Post-Intervention Psychosocial Mediator Surveys

Post-Intervention Resting Heart Rate

Post-Intervention Resting Blood Pressure

$\mathrm{X}_{\text {POST }}=\quad$ Control Group will receive exercise intervention after study completion 


\section{Participants}

The participants recruited for this study were required to be between the ages of 60-90 years old with no diagnosed neurological defect (e.g., possible dementia, self-report of neurological disease such as multiple sclerosis, brain tumor, and Parkinson's disease) and were willing and able to participate in an aquatic exercise program. They received medical clearance from their physician denoting that they were permitted to participate in regular, aerobic exercise sessions and completed an exercise field test. Participants were not currently involved in regular

exercise training (e.g., running, cycling, swimming, weightlifting, etc.) defined as greater than 30 minutes of such activities greater than 5 times per week (150 minutes in total). Internal review board (IRB) approval was received prior to beginning recruitment for participants.

Forty-four $(\mathrm{N}=44)$ participants were recruited by notice board flyers at the West Virginia University campus and Medical Centers/Clinics as well as inclusion in community bulletins (i.e., churches, senior centers, retirement homes, etc.). Prospective participants were asked to contact Lori Sherlock via phone or e-mail to receive the description of the study. If the potential participant was interested in taking part in the study, he or she was asked a number of questions during the phone conversation to gauge his or her eligibility for the study (Appendix B). If the individual met the initial criteria, he or she was invited to visit our laboratory to discuss the study in detail. The consent form was presented to the participant by Lori Sherlock for consent to participate during the onsite visit (Appendix A).

Eligible participants $(\mathrm{N}=44)$ were arranged into matched pairs by utilizing age and fitness parameters. One participant from each pair was then randomly assigned to either the treatment group to take part in the 10-week aquatic exercise intervention or the control group. Members of the treatment group received cognitive and fitness assessments as well as 10-weeks 
of an individualized, expert-instructed, aquatic exercise program. Members of the control group received the same cognitive and fitness assessments and were offered the same 10-week program following completion of the study. Of the 44 participants that initially began the testing battery, $34(n=34)$ completed the research study in full $(n=17$ in both the intervention and control groups).

\section{Treatments}

The study was comprised of 3 pre-intervention visits, a 10-week aquatic group fitness class, a post-intervention visit, and a post-intervention focus group. All participants in the treatment group were asked to take part in each component of the study. Each of the three preliminary visits addressed specific study needs and aims for appropriate data collection. Separation of the pre-intervention test battery into 3 detached visits was necessary to provide either familiarization for increasing test validity, reduction of respondent fatigue, or appropriate test site provisions.

\section{Preliminary Testing}

Visit 1 consisted of familiarization of the Cambridge Neuropsychological Test Battery (CANTAB®), completion of a health history, collection of demographic data, and completion of the past year total physical activity questionnaire (PYTPAQ) three weeks prior to the intervention. $\mathrm{CANTAB}{ }^{\circledR}$ is software that allows computerized cognitive screening for a variety of cognitive parameters utilizing a touch-screen laptop and press pad. The CANTAB ${ }^{\circledR}$ manufacturers and scientists recommend that all participants in research projects perform a familiarization session utilizing a practice test one week prior to the administration of the baseline test battery. The practice test allows the participant to become comfortable with the testing materials and administration procedures to secure more valid baseline results. The health 
history and demographic data provided valuable information on the participant's medical status and history. This information was collected as a safety precaution to improve the researchers' awareness of any pre-existing medical conditions or contraindications and precautions for exercise. Demographic data and information collected from the health history also provided further insight towards answering the research questions or identifying relations between variables. Lastly, the PYTPAQ is a survey devised to determine participant's level of physical activity prior to the intervention.

The second visit took place 2 weeks prior to the onset the aquatic exercise intervention. During this visit, anthropometric measurements including height, weight, hip-to-waist ratio, and body composition assessment using the Bod Pod and triceps skin fold, collection of participant's medical release provided by their personal physician, resting heart rate, and blood pressure were collected. Completion of 3 surveys on exercise barriers, social support, self-efficacy, and motivators were also secured. Cognitive screening using the CANTAB ${ }^{\circledR}$ was conducted to assess working memory capacity, spatial working memory, orientation, registration, attention, calculation, language and recall. The pre-intervention anthropometric measurements, resting heart rate and resting blood pressure, provided a baseline for comparison to the post-intervention measurements. These parameters are noted to change with improved fitness and can have a positive effect on quality of life. The psychosocial mediator surveys contributed to the researchers' knowledge of the participant's current point of view on exercise barriers, social support, self-efficacy, and motivators.

Visit 3 occurred the week prior to the onset of the exercise intervention. During the third visit, participants were asked to complete a 6-minute walk test. They were instructed to 
complete the test as quickly as they could by walking. Heart rate, blood pressure, and RPE were taken prior to the test and immediately following.

\section{Intervention}

After all preliminary data were compiled; the treatment group began the aquatic exercise intervention. The treatment consisted of a 10-week intervention period involving a 5-10 minute warm up followed by 50 minutes of moderate intensity aerobic exercise and concluded with a 510 minute cool down performed in an aquatic environment 3 times per week. Participants exercise sessions were graded from $45 \%$ heart rate reserve (HRR) increasing to $75 \%$ HRR over the course of the trial utilizing both continuous and interval-based training designs (Appendix D). Intensity was determined by the preliminary exercise testing and observed via the use of heart rate monitors set to participant's individual parameters in conjunction with the Kruel formula for aquatic target heart rate. Certified aquatic exercise instructors led the group fitness class and provided participants with both demonstration and cues for the exercises being performed. Adult learning theory was infused into the instruction methods during the classes to promote greater understanding (see subsequent section on Adult Education). Heart rate and rate of perceived exertion were monitored throughout each exercise session via heart rate monitors and data collection sheets. At week-5 of the exercise intervention (mid-intervention), participants were given 2 questionnaires to assess if barriers, social support, motivation, or selfefficacy had changed.

\section{Post-testing}

At the conclusion of the 10-week exercise intervention, both treatment and control group participants were asked to join in a final visit. The visit took place during the week immediately following the conclusion of the exercise intervention. The post-intervention visit consisted of 
collecting participant's anthropometric measurements including weight, hip-to-waist ratio, and body composition assessment using the Bod Pod and triceps skin fold, resting heart rate, resting blood pressure, noting any changes in health history, and completion of 3 surveys on exercise barriers, social support, self-efficacy, and motivators. Cognitive screening using the CANTAB ${ }^{\circledR}$ was conducted to reassess working memory capacity, spatial working memory, orientation, registration, attention, calculation, language and recall. Lastly, participants were asked to complete a 6-minute walk test. They were instructed to complete the test as quickly as they could by walking. Heart rate, blood pressure, and RPE were taken prior to the test and immediately following.

\section{Data Collection}

All volunteers were asked to participate in every component of the study. As described previously, there were 3 preliminary visits, a 10-week aquatic exercise intervention, and a postintervention visit. A post-intervention focus group was also conducted with the intervention group as a means of enriching the researcher's knowledge on the participant's feelings towards exercise, barriers, goals, social support, and relation of exercise to activities of daily living (ADLs) as well as cognitive function. Two focus groups were conducted to allow for size equability and participant ease. The focus groups were guided by an unbiased mediator and recorder to reduce the threats of data collection bias. They were recorded via audio tape and digital audio recorder in addition to the on-site recorder taking notes on the sessions.

\section{Procedures.}

\section{Cambridge Neuropsychological Test Battery (CANTAB). The Cambridge}

Neuropsychological Test Battery is a well-validated cognitive testing tool (Verhey et al., 2003; Ritsner, Blumenkrantz, Dubinsky, \& Dwolatzky, 2006). Establishment of construct validity of 
CANTAB has been accomplished testing subjects with neurological diseases with known brain focal effects as well as conducting comprehensive comparisons with traditional neuropsychological tests (Jazbec et al., 2007; Owen, Roberts, Polkey, Sahakian, \& Robbins, 1991; Barnett et al., 2010; Swainson et al., 2001; Gau \& Shang, 2010). It is sensitive to small changes in various cognitive domains and is culturally independent (Verhey et al., 2003; Ritsner et al., 2006). All of the tests are performed using touchscreen technology and are designed to assess changes in executive function parameters.

Participants completed pre and post cognitive function tests utilizing a Lonovo ThinkPad X230 Tablet touch-screen computer fitted with a Press Pad V2 (Cambridge Cognition). Participants sat with the ThinkPad at a comfortable distance in front of them (approximately 1.5 feet). Participants were familiarized with the $\mathrm{CANTAB} \AA$ one week prior to the actual test administration, as recommended by the Cambridge Cognition manufacturer and scientists. The familiarization session consisted of induction tests that introduced the participant to the touchscreen technology. These preliminary tests screened for visual, movement, and comprehension difficulties to alert the researcher of the participant's ability to comply with the testing parameters. Participants unable to complete the preliminary tests were omitted from the study due to their inability to complete the actual CANTAB® tests successfully $(n=1)$.

Following the familiarization session, participants cognitive function baseline measurements were assessed with five separate tests: (1) one touch stocking of Cambridge (OTS), (2) paired associates learning (PAL), (3) spatial working memory (SWM), (4) attention switching task (AST), and (5) reaction time (RTI). Each test included in the battery evaluated varying components of executive function (spatial planning, working memory, visual memory, 
new learning, age related memory loss, spatial memory, manipulation of working memory, and latency) and provided a variety of outcome measures (see table 4.3).

One touch stockings of Cambridge (OTS) necessitates participants evaluate the number of modifications needed to match a corresponding pattern. During the OTS assessment (Figure 3.2), participants are shown two displays containing three colored balls. The balls are held in stockings (socks or pockets) and are stacked atop one another. There are also a row of boxes containing numbers displayed at the bottom of the screen. Participants are given verbal instructions to make the bottom ball arrangement mirror the top ball arrangement by moving the balls one at a time. Verbal instructions are followed by a demonstration while repeating the instructions. The participants are given four examples prior to entering the assessed phase of the test to assure full understanding and to allow practice.

Figure 3.2: OTS Assessment

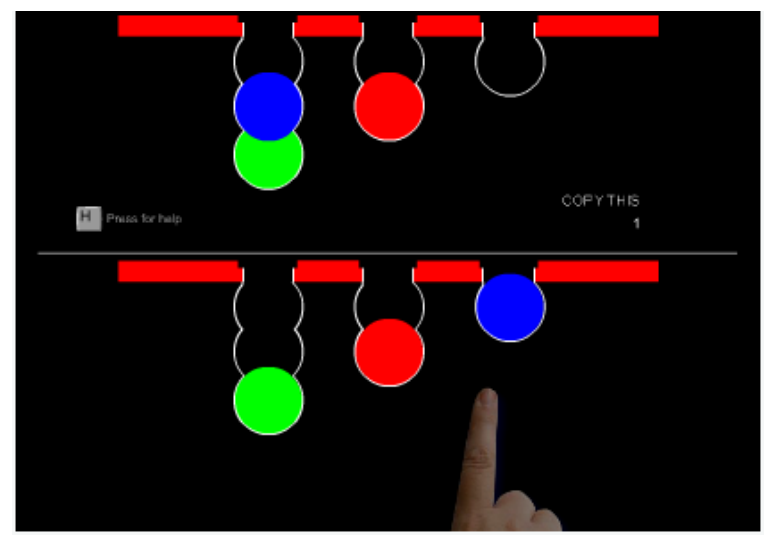

The paired associates learning (PAL) (Figure 3.3) test requires participants to learn and remember pattern-location associations. The assessment shows participants a set of abstract patterns in a set of six or eight boxes then, after a brief delay, presents one of the now hidden patterns in the center of the screen. The participant is required to touch the box where they saw the pattern appear. If the participant selects the correct box, the process is repeated with a 
different pattern, if an incorrect selection is made, the participant is shown the hidden patterns once more. The test begins with verbal instructions and a demonstration followed by a practice session for complete understanding of the test objective. This process has been shown to allow for assessment of early stages of memory impairment via visuo-spatial association (Junkkila, Oja, Laine, \& Karrasch, 2012).

Figure 3.3: PAL Assessment

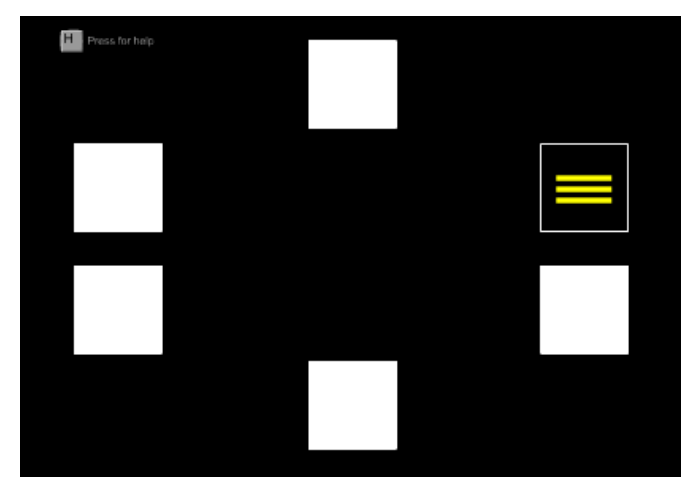

Spatial working memory (Figure 3.4) is the ability of the participant to actively manipulate spatial information in the working memory. During the SWM assessment, colored squares are shown on the screen. The participant is asked to find a blue 'token' in each of a series of boxes and use the 'tokens' to fill up a column on the right side of the screen. The numbers of boxes are gradually increased from three to eight and the position of the boxes change from trial to trial. As in previous tests, verbal instructions, a demonstration, and participant practice is provided prior to the assessed phase. The SWM test is a non-verbal, selfordered task that assessed both strategy and between errors. Strategy is scored by obtaining the number of times the participant begins a new search for a hidden 'token' during the assessed portion of the test. It is suggested that a proficient strategy for completing the SWM task is to devise a sequence beginning with a specific box and then, once a token has been found, to return to that box to begin the search for the next hidden token (Owen, Downes, Sahakian, Polkey, \& 
Robbins, 1990). This sequencing plan, or strategy, assists the participant in organizing the search for the tokens and completing the task in an efficient manner. Due to the necessity of strategic planning to achieve an optimal score, this test has been shown to be sensitive to cognitive dysfunction disorders (Joyce, Blumenthal, \& Wessely, 1996).

Figure 3.4: SWM Assessment

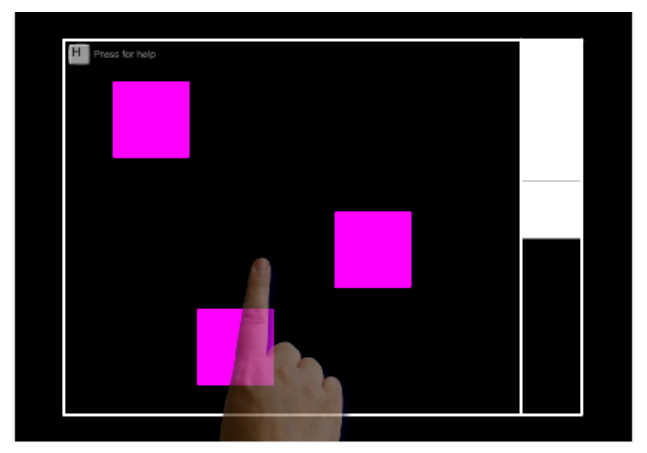

The AST (Figure 3.5) involves the use of the Cambridge Cognition Press Pad V2 placed in front of the computer. The press pad has 2 buttons on it, one to the right and the other to the left. During the test, an arrow will appear on the screen that points either left or right. Depending on the stage of the assessment, the participant will see the instruction "Which Direction?" or "Which Side?" prior to each problem. The participant will then press the button that corresponds with the given instructions. There are four practice phases leading up to the assessed phase of this test. The objective of this test is to gauge the participant's ability to ignore task-irrelevant information and switch attention between the location and direction of the arrow therefore assessing sensory processing of stimuli. 
Figure 3.5: AST Assessment

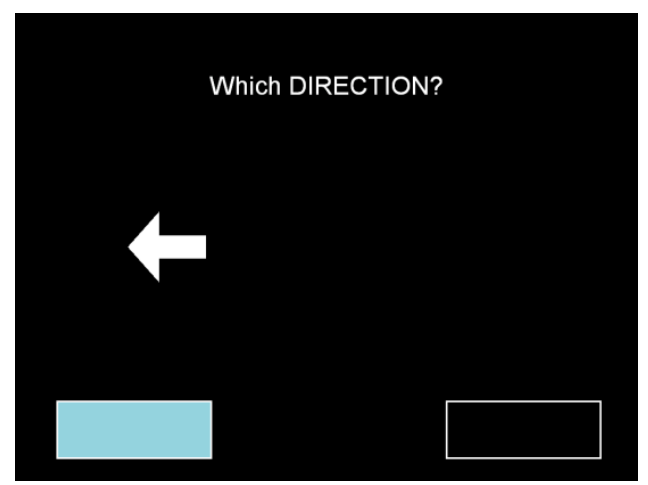

Lastly, during the RTI test (Figure 3.6), participants are asked to hold the button on the press pad down until they see a yellow dot appear on the screen. They are then instructed to release the press pad button and tap the yellow dot with their index finger as quickly as possible then return their finger to the button. There are two practice phases prior to the assessment portion of the RTI test.

Figure 3.6: RTI Assessment

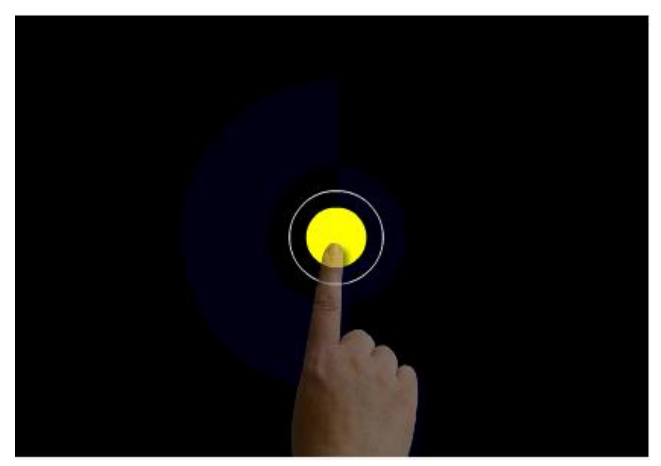

Health History. A comprehensive health history (Appendix C) interview was conducted privately with each participant to determine their initial health status, past and present medical issues, and identify risk factors as well as contraindications and precautions pertaining to aquatic exercise. The health history was imperative for providing safe and effective exercise prescription parameters to each, individual participant. The health history utilized for this study 
includes: biographical data, history of illness, injury and hospitalizations, family medical history, medication status, psychological health, and substance use.

Demographic Data. The demographic data (Appendix C) that was collected from each participant included sex, marital status, birthdate, education level, and occupational information. This information will provide the researcher with variables to consider when assessing the data collected. Demographic variables have been shown to play a role in cognitive function and thus they must be ascertained and considered in data analysis.

Past Year Total Physical Activity Questionnaire (PYTPAQ). All participants took part in a Past Year Total Physical Activity Questionnaire interview. The PYTPAQ measures all parameters of physical activity (frequency, duration, and intensity) and is broken into three sections that assess household, occupational and recreational physical activity within the previous 12 months. Participants estimated the average amount of time per week spent for a given activity in each of the three sections of the survey. Each activity was assigned a metabolic equivalent (MET) value, obtained from the Compendium of Physical Activities (Ainsworth et al., 1993). The assigned MET value was then multiplied by the total hours per week spent in each activity, thus physical activity was expressed as MET-hours/week.

Though the researcher completed the PYTPAQ with the participants, one or more of the responses provided by the participants were incomplete, thus full calculation of the MET values could not be achieved for the entire sample. Reasons for this included responder fatigue and time allocation for the total visit time. The PYTPAQ was not a critical variable but intended as a predictor variable in relation to the 6 minute walk test. For these reasons, the results from the PYTPAQ were omitted from the study analysis. 
Anthropometric Measurements. Anthropometric measurements are comparative measurements of the body that can be used as assessment tools and compared to reference standards. The comparison allows for risk stratification for various diseases as well as illustration of improvements in certain physical parameters associated with fitness. In this study, the anthropometric measurements taken included: height, weight, hip-to-waist-ratio, and body composition (Appendix E). These measurements were taken at baseline (one week prior to beginning the aquatic exercise intervention) and at the completion of the 10-week intervention. Anthropometric measurements can act as a gauge to fitness and disease risk. Collecting both baseline and post-intervention anthropometric measurements permitted the researcher to compare preliminary fitness parameters as well as risk for disease. Relations between these parameters and cognitive function were then compared and contrasted. Baseline measures also allowed for comparison between the treatment and control groups to assess normal distribution and homogeneity between groups.

Resting Heart Rate. Resting heart rate is the number of times the heart beats in one minute. Many factors can influence heart rate including: fitness, activity level, medications, and a variety of environmental and homeostatic elements. Resting heart rate (RHR) was assessed via palpation of the radial pulse for 30 seconds after the participant has been seated for 10 minutes. The 30-second pulse count was then multiplied by two, providing the RHR in beats per minute (bpm). In addition to the palpation method, pulse pressure (PP) was calculated from SBP-DBP measured with a validated, automated, oscillometric, sphygmomanometer (Omron M5-I, Omron Healthcare, Kyoto, Japan). Pulse wave analysis was performed noninvasively on the radial artery (SphygmoCor system, AtCor Medical, Sydney, Australia). All measurements were made in triplicate, and the mean values used for subsequent analysis. 
The RHR was a necessary measurement for multiple reasons; it implies the participant's level of fitness (if there are no other confounding variables), can provide diagnostic purpose for various arrhythmias, and it was a necessary component for the treatment group in order to configure target heart rate zones for the individualization of the exercise intervention.

Medications that are known to alter heart rate were taken into account when configuring participant's target heart rate zones.

Resting Blood Pressure. Blood pressure (BP) is the measure of the force of the blood against the arterial walls. Systolic BP, or the top number, is a reading of the vessel pressure as the heart beats (work). Diastolic BP, the bottom value, is a measure of the vessel pressure between beats (rest). Resting blood pressure is a valuable physiologic measurement for a variety of reasons. It is highly linked to complications in the heart, vessels, brain, kidneys, eyes, as well as general somatic function. Risk for hypertension, or high BP, increases with age and has been linked to reductions in cognitive function.

Each participant had a resting BP taken pre and post intervention as both a precautionary measure and as a fitness measurement. Though BP is not a direct measurement of fitness, exercise has been noted to cause positive adaptations to occur in the cardiovascular system allowing for an overall reduction in resting BP.

Participants were asked to remain seated for two minutes. To take the resting BP, the researcher employed the use of a sphygmomanometer and stethoscope, assuring the use of an appropriately sized cuff depending on arm girth. If the BP reading needed to be repeated due to errors, a period of 30-60 seconds was allotted between trials to allow for return of normal circulation. In addition, brachial systolic (SBP) and diastolic (DBP) blood pressure was measured with a validated, automated, oscillometric, sphygmomanometer (Omron M5-I, Omron Healthcare, Kyoto, Japan) and pulse pressure (PP) was calculated from SBP-DBP. Pulse wave analysis was 
performed noninvasively on the radial artery (SphygmoCor system, AtCor Medical, Sydney, Australia). All measurements were made in triplicate, and the mean values used for subsequent analysis. The same procedures were followed during the post-intervention data collection.

Psychosocial Mediator Surveys. Participants were asked to complete 3 surveys on exercise barriers, social support, self-efficacy, and motivators (Appendix F). Survey number one consisted of 13 items relating to exercise barriers. The survey was adapted from an existing exercise barriers survey that asks participants to indicate how often each of the 13 items interferes or prevents exercise participation (Zizzi, 2012). It was a self-administered survey that utilized a 1-5 ( $1=$ never, $5=$ very often $)$ likert scale. The second survey allowed the participants to rate their personal motivators for exercise. Utilizing a 1-6 $(1=$ not at all important, $6=$ very important) likert scale, participants ranked each of the 6 items. This survey was created from information taken from the literature that deciphers the most common motivators related to exercise in older adults. The final survey consisted of one question pertaining to social support and eleven items pertaining to self-efficacy. This survey was an adaptation and conglomeration of four existing surveys (Zizzi, 2012; Marcus, Selby et. al, 1992; Resnick \& Jenkins, 2000; McAuley, 1992).

The participants were given time to complete the surveys during their second visit (pre), following an exercise session during the fifth week of the exercise intervention (mid), and during their final visit (post). Pre, mid and post surveys were compared to denote any alterations in barriers, social support, self-efficacy, and motivators throughout the course of the intervention.

The researcher elected to survey the selected psychosocial mediators (barriers, social support, self-efficacy, and motivators) based on findings in the current literature. Older adults have been noted to have very specific barriers and motivators to exercise that are a signature to their specified age group. Barriers and motivators are interlinked and reflect one another for 
individuals over the age of 60 . Barriers related to health status and physical ability levels of the aging population are also the most commonly denoted motivators for participation in exercise. Self-efficacy, or the belief in one's own ability to succeed, has been noted as one of the strongest physical activity participation correlates in older adults (Bauman et al., 2012). Social support has also been indicated to have a very strong association with physical activity levels in this population (Bauman et al., 2012; SJH Biddle et al., 2012). For these reasons, barriers, social support, self-efficacy, and motivators were the primary focus concerning psychosocial mediators.

6-Minute Walk Test. The 6-Minute Walk Test (Appendix G) is one of the most common field tests used to assess cardiorespiratory and functional fitness. It should also be noted that a walking test, such as this, may be the most appropriate type of test for older adults given the reduction of joint-related stress that occurs during the testing procedure (Rikli R.E. \& Jones C.J., 1998). The 6-Minute Walk Test was used as a predictor of aerobic fitness. This test was completed during the third visit, one week prior to the exercise intervention. Heart rate and blood pressure was taken prior to beginning the test. Participants were then asked to walk for 6 minutes as quickly as possible without jogging or running. The researcher kept time with a stopwatch, record the total distance completed, and re-administered heart rate and blood pressure readings immediately following the test. Once these measurements were taken, participants were instructed to walk for a few minutes at a very easy pace to allow the body to return to preexercise levels. Distance was converted from feet to meters and the participant's predicted $\mathrm{VO}_{2 \mathrm{MAX}}$ was calculated utilizing the following equation (Swisher AK \& Goldfarb AH, 1998): VO2 $(\mathrm{mL} \cdot \mathrm{kg}-1 \cdot \mathrm{min}-1)=20.05+(0.019 \times$ Distance Walked in meters $)-(0.275 \times \%$ body fat $)$ 
The 6-Minute Walk Test was performed prior to and following the 10-week intervention to assess any changes in cardiorespiratory fitness. Positive alterations in cardiovascular fitness have been highly correlated to improvements in cognitive function. The researcher also investigated the relation of alterations in fitness with psychosocial mediators and focus group outcomes.

Focus Group. A post-intervention focus group was moderated and audio-recorded at the conclusion of the intervention. The focus group was conducted with the treatment group only and concentrated on attitudes, barriers, and relation of exercise to ADLs and cognitive function. During the focus group session, an introduction was recited to the participants expressing the importance of everyone contributing their input to the discussion, ability to speak freely, and allowing others to finish commenting prior to interjecting. The intended goals of the meeting as well as ground rules were also reviewed. The group moderator guided the discussion points throughout the course of each focus group while the recorder took notes and record the session's outcomes. Both sessions were recorded via audio tape recorder as well as a digital audio recorder to ensure the entirety of the focus group was captured.

The focus group interview was semi-structured with open-ended questions and probing questions to elicit greater or more complete responses (Appendix H). Each group consisted of no more than 10 individuals $(n=7, n=7)$, thus multiple focus groups were necessary. Group members received a handout with bulleted topics for discussion prior to the focus group session. Focus groups were then conducted in a neutral, comfortable environment that promoted a casual atmosphere and were conducive to conversation as well as group interaction.

The outcomes of the focus group were transcribed and sorted to enrich the quantitative components of the study. During transcription, pseudonyms were used to allow for maintenance 
of participant confidentiality. Participants will be referred to in accordance with their given pseudonym in relation to the transcript with one exception. The pseudonym of "Pam" was given for a participant in focus group A as well as in group B, thus "Pam" from focus group A will be referred to as "Pamela" and "Pam" from focus group B will be denoted as "Pam."

The qualitative component permitted a more complete view of the effects of the exercise intervention in relation to the participant's attitude, barriers, relation of exercise to ADLs, and cognitive function.

Adult Education. The role of adult education in behavior change is well noted throughout the literature. Some theories of adult learning and education accentuate the importance of beginning with small, reinforced modifications that gradually lead to a more complex behavioral pattern alteration (Skinner, 1953; Bandura, 1986). This step-wise approach parallels the means of progressively incorporating physical activity into an individual's lifestyle or improving general fitness. During this study, the progression during the aquatic-based exercise intervention modeled this theory by utilizing progressive overload. Progressive overload is the concept of gradually increasing frequency, intensity, and duration of training until the desired fitness level is achieved. Though frequency will remain consistant at 3 days per week, the intensity and duration of the aquatic exercise bout will be modified to gradually build the fitness base of the participants. By eliciting gradual modifications in exercise behavior and reinforcing that behavior with intrinsic and extrinsic motivators (i.e., positive feedback and successful completion of a session), the researcher anticipated a more effective and lasting behavioral change.

This study also included an educational component during the exercise instruction to further elicit the power of adult education to assist in behavior change. Including the reasoning 
for performing the specific exercises (i.e., improved function, reduction of injury susceptibility, etc.) may assist in solidifying a more lasting behavioral change (Health Belief Model, Becker, 1974; Hochbaum, 1958; Rosenstock 1960, 1966, 1990), while further education on the performance of the exercise techniques or movements may increase self-efficacy (Theory or Reasoned Action, Theory of Planned Behavior \& Social Cognitive Theory, Ajzen, 1985, 1988; Bandura, 1977). The researcher anticipated that by integrating a variety of educational components into the exercise sessions, the adult learners needs and interests were highlighted yeilding an applicable learning experience that translated into participation in other physical activities. Lastly, the social component of the aquatic exercise intervention was promoted and fostered. Social support is commonly associated with health-related behaviors including physical activity and increasing the social component of the group fitness class may have assisted the treatment group in fostering greater social support from their exercise cohort.

\section{Data Analysis}

Quantitative Data. The quantitative data was analyzed using SPSS software (version 21.0, SPSS Inc., Chicago, Illinois) for normal distribution and homogeneity. The data were normally distributed, thus parametric statistics were used to determine the main effects and interactions between the intervention and control groups. A two-way analysis of variance (ANOVA) with a group (treatment versus control) by time (pre and post intervention) was utilized to examine the quantitative research questions, numbers one through four, for efficiency and error rate reduction (Gay, G.E.Mills, \& P.Airasian, 2006).

Qualitative Data. The qualitative data collected from the focus group was analyzed to assist in further informing the researcher on the attitudes, barriers, or relation of exercise to ADLs or cognitive function. The analysis of the data was based on recommendations of Patton 
(2002), endorsing careful data review to identify emergent themes. The focus groups were recorded via a tape recorder and digital voice recorder and recordings were transcribed then checked against one another to assure the content was sound. During transcription, pseudonyms were used to allow for maintenance of participant confidentiality. Participants will be referred to in accordance with their given pseudonym in relation to the transcript with one exception. The pseudonym of "Pam" was given for a participant in focus group A as well as in group B, thus "Pam" from focus group A will be referred to as "Pamela" and "Pam" from focus group B will be denoted as "Pam." The researcher reviewed the focus group interview transcripts at least three times while making notes following an inductive and logical analysis pattern. While conducting the content analysis, the researcher employed both inductive and frequency analysis to discover emerging themes. Themes were coded to denote categories of meaningful findings, which were further distinguished for patterns, subcategories, or relations. After themes fell out, the researcher reread the transcripts for any negative or counter instances related to the themes to increase the trustworthiness of the qualitative findings. Qualitative findings were used for answering in part, RQ1-RQ4 and in whole RQ5-RQ6.

Triangulation. After individually addressing the quantitative and qualitative findings, method triangulation was performed with the quantitative and qualitative findings to assist in the truth value and trustworthiness of the qualitative findings while promoting validity of the quantitative data. Triangulation was used to assist in answering RQ1-RQ4.

Research Question One. Research Question One asks: Does participation in a 10-week aquatic exercise class result in a statistically significant $(\mathrm{p} \leq .05)$ improvement in cognitive function when compared to the control group? The null hypothesis for Research Question One $\left(\mathrm{H}_{01}\right)$ states: Participation in a 10 -week aquatic exercise class does not result in a statistically 
significant $(\mathrm{p} \leq .05)$ improvement in cognitive function when compared to the control group.

The researcher utilized the CANTAB ${ }^{\circledR}$ system for measuring several components of cognitive function. The system saved and stored all results from tests elicited which were exported into spreadsheets and analyzed using SPSS. Individual cognitive function parameters were assessed for treatment impact.

Research Question Two. Research Question Two asks: Does participation in a 10-week aquatic exercise class result in a statistically significant $(\mathrm{p} \leq .05)$ improvement in cardiovascular fitness when utilizing the Kruel target heart rate deduction to configure individualized target heart rate zones when compared to the control group? The null hypothesis for Research Question Two $\left(\mathrm{H}_{02}\right)$ states: Participation in a 10-week aquatic exercise class does not result in a statistically significant $(\mathrm{p} \leq .05)$ improvement in cardiovascular fitness when utilizing the Kruel target heart rate deduction to configure individualized target heart rate zones when compared to the control group. Data from the 6-Minute Walk Test was the primary method used to monitor changes in cardiovascular fitness. Blood pressure readings and heart rate responses taken during the 6-Minute Walk Test pre-intervention and post-intervention were compared to the control group utilizing a two-way ANOVA.

Research Question Three. Research Question Three asks: Do statistical significant ( $\mathrm{p} \leq$ $.05)$ positive changes occur for general fitness and wellness parameters after participation in a 10-week aquatic exercise class when compared to the control group? The null hypothesis for Research Question Three $\left(\mathrm{H}_{03}\right)$ states: No statistical significant positive changes $(\mathrm{p} \leq .05)$ occur for general fitness and wellness parameters after participation in a 10-week aquatic exercise class when compared to the control group. General fitness and wellness parameters were tested pre 
and post intervention via anthropometric measurements (weight and body composition), resting blood pressure, and resting heart rate.

Research Question Four. Research Question Four asks: Does participation in a 10week aquatic exercise class result in a statistically significant $(\mathrm{p} \leq .05)$ alteration in social support, self-efficacy, motivators, or exercise barriers when compared to the control group? The null hypothesis for Research Question Four $\left(\mathrm{H}_{04}\right)$ states: Participation in a 10-week aquatic exercise class does not result in a statistically significant $(\mathrm{p} \leq .05)$ alteration in social support, self-efficacy, motivators, or exercise barriers when compared to the control group. Data collection for Research Question Four consisted of 3 participant surveys administered pre, mid, and post intervention. Surveys asked participants about barriers, motivators, self-efficacy and social support related to exercise. Responses from each of the surveys were appraised. Focus group transcripts and notes were also reviewed for immerging themes concerning psychosocial mediators to further enrich the findings for Research Question Five.

Research Question Five. Research Question Five asks: Does the infusion of adult education theory into a 10-week aquatic exercise class assist in behavior change? Focus group transcripts and notes were reviewed for immerging themes and analyzed qualitatively using the methods described by Patton (2002) concerning adult education theory and behavior change.

Research Question Six. Research Question Six asks: Does aquatic exercise provide participants with greater accessibility to exercise participation? Focus group transcripts and notes were reviewed for immerging themes and analyzed qualitatively using the methods described by Patton (2002) concerning exercise accessibility. 


\section{Chapter Four Findings and Discussion}

\section{Review of Purpose}

The primary purpose of this study was to investigate the relation between aquatic-based cardiovascular exercise and executive function in older adults. The study examined the effects of a10-week aquatic-based cardiovascular exercise program on cognitive and wellness parameters. Both quantitative and qualitative methods were employed to answer the six research questions posed by the primary investigator. The findings for each research question are organized by reporting the data relating to the impending question. RQ1-RQ4 utilizes quantitative data, followed by qualitative data and concludes with triangulation of the two methods. RQ5 and RQ6 use only qualitative findings to answer the imposed research questions.

\section{Review of Research Questions}

RQ 1: Research Question One asks: Does participation in a 10-week aquatic exercise class result in a statistically significant ( $p \leq .05)$ improvement in cognitive function when compared to the control group?

This question addresses the concern associated with cognitive function decline connected to aging. Due to the rapid increase in the aging population and the global effects that cognitive function has on successful aging, the discovery of preventative strategies for cognitive decline are paramount. Research focused on land-based exercise has presented positive outcomes with both prevention and protection against neurocognitive decline (Park \& Reuter-Lorenz, 2009). Aligning aquatic exercise with these findings holds importance due to the common physical decline associated with the aging process. Physical decline, whether it is reduction in strength, compromised joint structure, or poor balance, may increase risk associated with land-based exercise and thus hinder individuals from participating in regular physical activity. The aquatic environment provides support in the form of buoyancy and viscosity to allow the immersed body to move safely and, often, with less movement-associated pain. These distinguishing features of 
the aquatic environment may make exercise more accessible, in participation, duration, and intensity, to the aging population.

RQ2: Does participation in a 10-week aquatic exercise class result in a statistically significant ( $p \leq .05)$ improvement in cardiovascular fitness when utilizing the Kruel target heart rate deduction to configure individualized target heart rate zones when compared to the control group?

While investigating the alignment of exercise in the aquatic environment land-based exercise, a parallel relation needs to be established between these exercise parameters. Moderate to intense land-based exercise has been well supported for improvement in cognitive function parameters in congruence with improvement in cardiovascular fitness (Angevaren et al., 2010) Due to these findings, it is necessary to assert that the appropriate intensity is being met and the proper stimulus is achieved in order to improve cardiovascular fitness and thus cognitive function. With this aim in mind, the realization that the aquatic environment imposes a potentially noteworthy (up to 17\%) downward effect on heart rate at rest and during exercise needs to be factored in to the training parameters (Becker BE \& AJ Cole, 2011). Utilization of the Kruel target heart rate deduction will allow for water-based exercise to align with land-based training on target heart rate goals to achieve the intensity recommendations documented in the literature (Kruel, 2009).

RQ3: Do statistically significant ( $p \leq .05)$ positive changes occur for general fitness and wellness parameters after participation in a 10-week aquatic exercise class when compared to the control group?

Fitness and wellness parameters are also being investigated to further decipher the global effects of the aquatic exercise intervention. These fitness factors may reveal a more specified relation to cognitive function and changes in one or more of the wellness parameters. They also may provide a more comprehensive view of overall wellness in relation to cognitive function.

RQ4: Does participation in a 10-week aquatic exercise class result in a statistically significant ( $p \leq .05)$ alteration in social support, self-efficacy, motivators, or exercise barriers when compared to the control group? 
Research question four relates to the individual's reasoning for beginning exercise as well as their aptitude for continuing exercise. The level of importance of these psychosocial mediators in participation and adherence to exercise parallels the significance of continuing exercise for sustained physical and cognitive benefits.

RQ5: Does the infusion of adult education theory into a 10-week aquatic exercise class assist in behavior change?

The importance of including educational components into the exercise prescription for the aging adult has been demonstrated in the literature (Bauman et al., 2012; Sallis JF et al., 2008; SJH Biddle et al., 2012; SJH Biddle et al., 2012). The variation of providing adult-specific educational strategies during the aquatic exercise intervention to promote a more permanent behavior change was examined due to the necessity of exercise maintenance.

RQ6: Does aquatic exercise provide participants with greater accessibility to exercise participation?

Exercise accessibility, as previously mentioned, is of great concern when working with an aging population. If exercise is not safe, or perceived to be safe, then participation will not be as probable. Pain has been noted as one of the primary contributors to a sedentary lifestyle in the elderly (Booth et al., 1997; Cohen-Mansfield, 2003; Schutzer \& Graves, 2004). The qualities of the aquatic environment can reduce or eliminate pain as a restrictive factor to exercise participation. Due to these inherent factors associated with water immersion, accessibility to exercise may be improved and therefore should be considered.

\section{Participant Attrition and Attendance}

Forty-four individuals meeting the inclusion criteria initially volunteered to participate in this study. Of the 44 individuals, 34 (17 intervention and 17 control subjects) completed the study in full denoting an attrition of $20.9 \%$ from the start of the study to completion. Eight of the 
nine individuals that did not complete the study dropped out on their own accord while one was asked not to continue due to a language barrier unsuitable for the cognitive function testing. Reasoning for attrition was noted to fall under two underlying circumstances: (1) medical reasons $(44.4 \%)$, and (2) time constraints $(44.4 \%)$.

Attendance for the intervention group showed a mean of $27.2(\mathrm{SD}=2.84)$ visits out of a possible 30 visits. This displays that the average participant attended approximately $91 \%$ of the classes. Mean participant absence was calculated to be 2.8 sessions $(\mathrm{SD}=2.8)$. Reasons recorded for absences included illness, appointments, family obligations, and vacation.

\section{Review of Data Collection and Analysis}

Preliminary and post intervention tests including executive function (CANTAB), 6-

minute walk, psychosocial mediator surveys, resting heart rate, blood pressure, arterial function, and anthropometric measurements were collected from both the control and intervention groups. The intervention group also participated in a mid-intervention psychosocial mediator survey as well as a post-intervention focus group. All measurements were taken under similar conditions: environment, testing order, and timing intervals for all participants. The pre-post data was analyzed using a two-way analysis of variance (ANOVA) and SPSS software (version 21.0, SPSS Inc., Chicago, Illinois).

Frequency and inductive analysis were conducted with the qualitative data. After the two recorded focus groups with the intervention group were concluded, the audio-recordings were transcribed and the transcriptions were proofed against (by listing to) the audio-recordings. The researcher became immersed in the data by listening to audio-recordings and through multiple readings of the transcriptions. During this process, notes were taken on common concepts and ideas and a framework emerged; data were indexed within and between subjects, organizing 
quotes and concepts categorically, and finally converging the interpreted data to identifiable themes.

Initial transcripts were created for each focus group (morning and afternoon exercise groups) and subcategorized by the interview question preceding the participants recorded responses. Index cards were utilized to further organize the data under codes and concepts inductively while noting frequency. Concepts and categories were then determined based on coding derived from participant's responses resulting in the creation of several higher-order themes for the intervention group.

\section{Focus Groups}

A focus group was conducted with the intervention participants. Two sessions were offered in accordance with the participants' aquatic exercise intervention time of day (morning or afternoon) in attempt to increase convenience for participants, and accordingly, the total number of individuals participating in the focus group. The participants were given ample notice about the dates and times of the focus groups that would take place after their final aquatic exercise class. Due to work schedules and other noted commitments, seven of the 10 participants were able to attend the morning focus group session and all 7 individuals attended the afternoon session. The morning attendees consisted of 5 women and 2 men, while the afternoon group was comprised of 6 women and 1 man. Pseudonyms were used to allow for maintenance of participant confidentiality. Participants will be referred to in accordance with their given pseudonym in relation to the transcript with one exception. The pseudonym of "Pam" was given for a participant in focus group A as well as in group B, thus "Pam" from focus group A will be referred to as "Pamela" and "Pam" from focus group B will be denoted as

"Pam." Each session took place in a comfortable environment and lasted no more than one hour. 
A moderator (experienced in the conduct of focus groups) and recorder facilitated each session; the researcher was not present. The focus groups included dynamic participant-to-participant interaction guided by the moderator to emphasize the researcher's pre-determined questions (Appendix H). The full focus group transcripts can be found in Appendix I.

As previously stated, the researcher utilized focus group materials to further the understanding of the intervention effects on the participants. One of the primary goals of the intervention, outside of obtaining answers to the research questions, was to provide a potent catalyst for a more permanent behavior change. This aim is pertinent due to the impending need of continuation of exercise as a chronic, adopted, imbedded lifestyle choice. As the literature review presented, acquired somatic and cognitive improvements degrade if physical activity is terminated.

Focus group methodology was directly utilized to answer RQ5 through RQ6, however, emergence of qualitative findings permitted triangulation with RQ1 - RQ4 as well. Method triangulation was performed for RQ1-RQ4 to further address the potential of the group's continuation with physical activity.

\section{Differences in Groups at Baseline}

Preliminary analyses were utilized to determine if the control and intervention groups had any statistically significant differences at their baseline measurements (table 4.1). Both the control and intervention groups on average presented with resting heart rates on the lower-end of what is considered to be the normal range (between 60-100 bpm), pre-hypertensive resting systolic blood pressures between 120-139 mmHg, normal resting diastolic blood pressures $(<80$ mmHg), and percent body fats well below average denoting a high rate of obesity (Farrell, 2011). The two-way ANOVA revealed that there were no statistically significant $(\mathrm{p} \leq 0.05)$ differences 
in the control and intervention groups at baseline in the parameters presented in table 4.1 .

Additionally, clinical characteristics of the participant cohorts revealed that $6 \%$ of the control group and $18 \%$ of the intervention group were diagnosed with Type 2 diabetes, $56 \%$ and $69 \%$ of the control and intervention group had cardiac issues respectively, and $69 \%$ of the control and $59 \%$ of the intervention group were hypertensive.

Table 4.1: Comparative Demographic Data for Control and Intervention groups

\begin{tabular}{|c|c|c|c|c|}
\hline & \multicolumn{2}{|c|}{ Intervention Group Demographics } & \multicolumn{2}{|c|}{ Control Group Demographics } \\
\hline $\begin{array}{l}\text { Sample Size } \\
(\mathrm{N}=34)\end{array}$ & $\mathrm{n}=17$ & & $\mathrm{n}=17$ & \\
\hline \multicolumn{5}{|l|}{ Gender } \\
\hline Male & $\mathrm{n}=3$ & & $\mathrm{n}=4$ & \\
\hline Female & $\mathrm{n}=14$ & & $\mathrm{n}=13$ & \\
\hline Mean Age & $67.71 \mathrm{y}$ & $\mathrm{SD}=7.14$ & $67.94 \mathrm{y}$ & $\mathrm{SD}=8.66$ \\
\hline \multicolumn{5}{|l|}{ Ethnic Background } \\
\hline White, not of Hispanic origin & $\mathrm{n}=14$ & & $\mathrm{n}=17$ & \\
\hline Black, not of Hispanic origin & $\mathrm{n}=2$ & & $\mathrm{n}=0$ & \\
\hline \multicolumn{5}{|l|}{ Marital Status } \\
\hline Married & $\mathrm{n}=10$ & & $\mathrm{n}=8$ & \\
\hline Single & $\mathrm{n}=0$ & & $\mathrm{n}=1$ & \\
\hline Widowed & $\mathrm{n}=5$ & & $\mathrm{n}=5$ & \\
\hline Divorced & $\mathrm{n}=2$ & & $\mathrm{n}=3$ & \\
\hline Mean Level of Education & $14.12 \mathrm{y}$ & $\mathrm{SD}=2.15$ & $13.71 \mathrm{y}$ & $\mathrm{SD}=2.47$ \\
\hline \multicolumn{5}{|l|}{ Occupational Status } \\
\hline Working & $\mathrm{n}=1$ & & $\mathrm{n}=2$ & \\
\hline Retired & $\mathrm{n}=16$ & & $\mathrm{n}=15$ & \\
\hline $\begin{array}{l}\text { Mean Resting Heart Rate } \\
(\text { Norm }=60-100 \mathrm{bpm})\end{array}$ & $64.76 \mathrm{bpm}$ & $\mathrm{SD}=7.03$ & $72.41 \mathrm{bpm}$ & $\mathrm{SD}=11.46$ \\
\hline $\begin{array}{l}\text { Mean Resting Systolic Blood Pressure } \\
\text { (norm < } 120 \mathrm{mmHg} \text { ) }\end{array}$ & $126.24 \mathrm{mmHg}$ & $\mathrm{SD}=15.02$ & $129.35 \mathrm{mmHg}$ & $\mathrm{SD}=19.48$ \\
\hline $\begin{array}{l}\text { Mean Resting Diastolic Blood Pressure } \\
\text { (norm }<80 \mathrm{mmHg} \text { ) }\end{array}$ & $68.71 \mathrm{mmHg}$ & $\mathrm{SD}=8.49$ & $66.41 \mathrm{mmHg}$ & $\mathrm{SD}=12.92$ \\
\hline Mean Distance Walked in 6 Minutes & $458.98 \mathrm{~m}$ & $\mathrm{SD}=106.35$ & $466.07 \mathrm{~m}$ & $\mathrm{SD}=79.39$ \\
\hline $\begin{array}{l}\text { Mean Percent Body Fat } \\
(\text { Average }=22.1-33 \%)^{*}\end{array}$ & $40.67 \%$ & $\mathrm{SD}=8.18$ & $40.78 \%$ & $\mathrm{SD}=7.71$ \\
\hline
\end{tabular}


In addition to the physiological assessments, the study sample participated in a

preliminary executive function test battery to assess baseline measurements for the control and intervention groups. The CANTAB testing battery was comprised of 5 assessments with individual scores displayed in Table 4.2 below. Two-way ANOVA revealed that no statistically significant $(\mathrm{p} \leq 0.05)$ difference in baseline executive function existed between groups for the chosen assessments.

Table 4.2: Cognitive Function Descriptive Statistics for Pre-Test Intervention and Control Group Participants

\begin{tabular}{|c|c|c|c|c|}
\hline Executive Function Test & \multicolumn{2}{|c|}{ Intervention Group } & \multicolumn{2}{|c|}{ Control Group } \\
\hline $\begin{array}{l}\text { One Touch Stockings of } \\
\text { Cambridge (OTS) } \\
\text { Problems Solved on } 1^{\text {st }} \text { Choice }\end{array}$ & 14.41 & $\mathrm{SD}=4.24$ & 15.25 & $\mathrm{SD}=2.52$ \\
\hline $\begin{array}{l}\text { Paired Associates Learning (PAL) } \\
\text { Total Errors }\end{array}$ & 32.35 & $\mathrm{SD}=25.29$ & 34.88 & $\mathrm{SD}=24.19$ \\
\hline $\begin{array}{l}\text { Spatial Working Memory (SWM) } \\
\text { Between Errors }\end{array}$ & 17.59 & $\mathrm{SD}=8.26$ & 19.65 & $\mathrm{SD}=7.76$ \\
\hline $\begin{array}{l}\text { Attention Switching Task (AST) } \\
\% \text { Correct Trials }\end{array}$ & $86.01 \%$ & $\mathrm{SD}=11.12$ & $89.15 \%$ & $\mathrm{SD}=9.63$ \\
\hline $\begin{array}{l}\text { Reaction Time (RTI) } \\
\text { 5-Choice Reaction Time }\end{array}$ & $391.98 \mathrm{~ms}$ & $\mathrm{SD}=46.80$ & $379.30 \mathrm{~ms}$ & $\mathrm{SD}=76.63$ \\
\hline
\end{tabular}

\section{Research Question One}

RQ 1: Research Question One asks: Does participation in a 10-week aquatic exercise class result in a statistically significant $(p \leq .05)$ improvement in cognitive function when compared to the control group?

Assessment of cognitive function was conducted utilizing a battery of executive-function focused tests prior to and following the 10 -week intervention period. The testing battery consisted of five separate tests: (1) one touch stocking of Cambridge (OTS) 6-choice-20, (2) 
paired associates learning (PAL) Parallel_pa2_SixAttempts2, (3) spatial working memory (SWM) Shortened-3X3P-2X4-40-2X6-60-2X8-80, (4) attention switching task (AST) 8d1-12d2$16 \mathrm{~s}-4 \mathrm{~s}-4 \mathrm{~d}-80 \mathrm{~s} 80 \mathrm{da}$, and (5) reaction time (RTI) parallel. Each test included in the battery evaluated varying components of executive function and provided an assortment of outcome measures (Table 4.3).

Table 4.3: Executive Function Battery Assessments, Parameters, and Outcome Measures

\begin{tabular}{|c|c|c|}
\hline Executive Function Test & $\begin{array}{l}\text { Executive Function Parameter(s) } \\
\text { Assessed }\end{array}$ & Outcome Measures \\
\hline \multirow{3}{*}{$\begin{array}{l}\text { One Touch Stockings of } \\
\text { Cambridge } \\
\text { (OTS) } \\
\text { 6-choice-20 }\end{array}$} & Frontal Lobe Function & Problems Solved on $1^{\text {st }}$ Choice \\
\hline & Spatial Planning & Mean Choices Correct \\
\hline & Working Memory & Mean Latency to $1^{\text {st }}$ Choice \\
\hline \multirow{3}{*}{$\begin{array}{l}\text { Paired Associates Learning } \\
\text { (PAL) } \\
\text { Parallel_pa2_SixAttempts2 }\end{array}$} & Visual Memory & Total Errors Made \\
\hline & New Learning & \\
\hline & Age Related Memory Loss & \\
\hline \multirow{5}{*}{$\begin{array}{l}\text { Spatial Working Memory } \\
\text { (SWM) } \\
\text { Shortened-3X3P-2X4-40- } \\
\text { 2X6-60-2X8-80 }\end{array}$} & Frontal Lobe Function & Errors Made \\
\hline & Executive Dysfunction & Strategy Score \\
\hline & Spatial Memory & Latency Measure \\
\hline & Working Memory Manipulation & \\
\hline & Heuristic Strategy & \\
\hline \multirow{3}{*}{$\begin{array}{l}\text { Attention Switching Task } \\
\text { (AST) } \\
\text { 8d1-12d2-16s-4s-4d- } \\
\text { 80s80da }\end{array}$} & Prefrontal Cortex & Percent Correct Trials \\
\hline & Top-Down Cognitive Control & Percent Incorrect Trials \\
\hline & Cognitive Flexibility & \\
\hline \multirow{4}{*}{$\begin{array}{l}\text { Reaction Time } \\
\text { (RTI) } \\
\text { parallel }\end{array}$} & Latency & Reaction Time \\
\hline & Movement time & Movement Time \\
\hline & & Accuracy Score \\
\hline & & Error Score \\
\hline
\end{tabular}


One Touch Stocking of Cambridge (OTS). One touch stocking of Cambridge (OTS) was measured using three outcomes: (1) problems solved in the first choice, (2) mean choices to correct, and (3) mean latency to first choice. The first outcome measure, problems solved in the first choice, provides the number of problems solved on the participant's first choice where a higher score denotes a better score. Mean values for the participants in the control and intervention group for the preliminary test were $14.41(\mathrm{SD}=4.24)$ and $15.25(\mathrm{SD}=2.52)$ respectively. The post intervention assessment produced mean values equaling $15.25(\mathrm{SD}=2.52)$ and $15.75(\mathrm{SD}=2.60)$ for the control and intervention group respectively and this difference was not statistically significant $(\mathrm{p}=0.26)$. A 2-way ANOVA was conducted and the interaction (time*group) and main effect for the group were not found to be statistically significant, however the time effect was statistically significant with a moderate power level $[F(1,31)=5.238, p=$ 0.028, ES $=0.147]$. These results denote a learning effect over time for both groups but show no change in relation to the intervention when comparing the intervention and control groups.

The second parameter measured by the OTS assessment was mean latency to correct. This measure utilizes an in-program algorithm to provide a mean latency, the time from the problem appearing on the screen until the time the correct ball is touched. A lower number denotes a better score with the results ranging from 1 and the number of balls displayed on the screen. The interaction between control and intervention groups was not found to be statistically significant and had a small associated power $[\mathrm{F}(1,32)=5.197, \mathrm{p}=0.170, \mathrm{ES}=0.058]$. The time effect, however, reached a level of statistical significance $(p=0.030)$ denoting that both groups experienced a learning effect for the task in relation to the time spent with selecting the correct pattern. 
The third and final factor measured by the OTS test was latency to first choice. This measure denotes the mean latency, or time elapsed, from the moment that the balls appeared on the screen to the instant the first ball was touched by the participant. The minimum number of moves needed to produce a correct pattern, as well as the number of times that the first choice was correct is factored into this result. A lower score signifies a better outcome. The main effect for time reached statistically significant levels $[F(1,32)=5.436, p=0.026, E S=0.145]$ but there were no other statistically significant findings within this measure.

This assessment, in its entirety, gauges the participant's executive function with an emphasis on the aspects of spatial planning and working memory. Table 4.4 shows the average value for each of these parameters. The overall outcomes of this measure showed no statistical significance for interaction between intervention and control groups over time. Time was the only variable that was statistically significant for each of the tested parameters ranging in statistical significance from $\mathrm{p}=0.017$ to $\mathrm{p}=0.028$.

Table 4.4: Mean Values for One Touch Stockings of Cambridge Outcome Measures

\begin{tabular}{|c|c|c|c|c|c|c|c|c|}
\hline & \multicolumn{4}{|c|}{ Control Group } & \multicolumn{4}{|c|}{ Intervention Group } \\
\hline & Pre & & Post & & Pre & & Post & \\
\hline $\begin{array}{l}\text { Problems } \\
\text { Solved in the } \\
1^{\text {st }} \text { Choice }\end{array}$ & 14.41 & $\begin{array}{l}\mathrm{SD}= \\
4.24\end{array}$ & 15.88 & $\begin{array}{l}\mathrm{SD}= \\
3.31\end{array}$ & 15.25 & $\begin{array}{l}\mathrm{SD}= \\
2.52\end{array}$ & 15.75 & $\begin{array}{l}\mathrm{SD}= \\
2.60\end{array}$ \\
\hline $\begin{array}{l}\text { Mean Latency } \\
\text { to Correct } \\
\text { (seconds) }\end{array}$ & $1.46 \mathrm{~s}$ & $\begin{array}{l}\mathrm{SD}= \\
0.50\end{array}$ & $1.31 \mathrm{~s}$ & $\begin{array}{l}\mathrm{SD}= \\
0.31\end{array}$ & $1.33 \mathrm{~s}$ & $\begin{array}{l}\mathrm{SD}= \\
0.18\end{array}$ & $1.30 \mathrm{~s}$ & $\begin{array}{l}\mathrm{SD}= \\
0.20\end{array}$ \\
\hline $\begin{array}{l}\text { Mean Latency } \\
\text { to First Choice } \\
\text { (milliseconds) }\end{array}$ & $14333.38 \mathrm{~ms}$ & $\begin{array}{l}\mathrm{SD}= \\
6835.96\end{array}$ & $11962.80 \mathrm{~ms}$ & $\begin{array}{l}\mathrm{SD}= \\
4376.78\end{array}$ & $13089.05 \mathrm{~ms}$ & $\begin{array}{l}\mathrm{SD}= \\
4788.43\end{array}$ & $11537.88 \mathrm{~ms}$ & $\begin{array}{l}\mathrm{SD}= \\
4803.05\end{array}$ \\
\hline
\end{tabular}

Paired Associates Learning (PAL). The paired associates learning (PAL) test requires participants to learn and remember pattern-location associations. This process has been shown to 
allow for assessment of early stages of memory impairment via visuo-spatial association (Junkkila et al., 2012). The PAL test provided a single, summative outcome measure of total errors. Errors are made in PAL when the participant chooses a box that does not contain the target pattern. Total errors denote the overall number of errors across the entirety of the assessed component of PAL thus lower scores indicate better results for this parameter (fewer mistakes made).

As noted in Table 4.5 below, post-intervention means were calculated to be 17.59 (SD = 13.51) for the control and $16.29(\mathrm{SD}=19.02)$ for the intervention group. There was no statistically significant interaction between intervention and control groups over time $[\mathrm{F}(1,32)=$ $0.306, \mathrm{p}=0.584, \mathrm{ES}=0.009]$. There was, however, a statistically significant time effect occurring from pre- to post-tests $[\mathrm{F}(1,32)=23.305, \mathrm{p}=0.000, \mathrm{ES}=0.421]$. Both the control and intervention groups average number of errors committed during the post-test were substantially lower than their pre-test scores representing a learning effect for the once novel assessment.

Table 4.5: Mean Values for Paired Associates Learning Outcomes

\begin{tabular}{|c|c|c|c|c|c|c|c|c|}
\hline & \multicolumn{4}{|c|}{ Control Group } & \multicolumn{4}{|c|}{ Intervention Group } \\
\hline & Pre & & Post & & Pre & & Post & \\
\hline Total Errors & 32.35 & $\mathrm{SD}=25.29$ & 17.59 & $\mathrm{SD}=13.51$ & 34.88 & $\mathrm{SD}=24.19$ & 16.29 & $\mathrm{SD}=19.02$ \\
\hline
\end{tabular}

Spatial Working Memory (SWM). Spatial working memory is the ability of the participant to actively manipulate spatial information in the working memory (Table 4.6 shows pre-post means). The SWM test is a non-verbal, self-ordered task that assessed both strategy and between errors. For the dependent variable of strategy, no statistically significant interaction between intervention and control groups over time was found $[\mathrm{F}(1,32)=2.16, \mathrm{p}=0.152, \mathrm{ES}=$ 
0.063]. Though no statistically significant finding were present, figure 4.1 shows an increase in strategy score for the control group (representing a reduction in performance) and a slight decrease in the intervention group (indicating improvement).

Figure 4.1: Pre-Post Spatial Working Memory Strategy Score

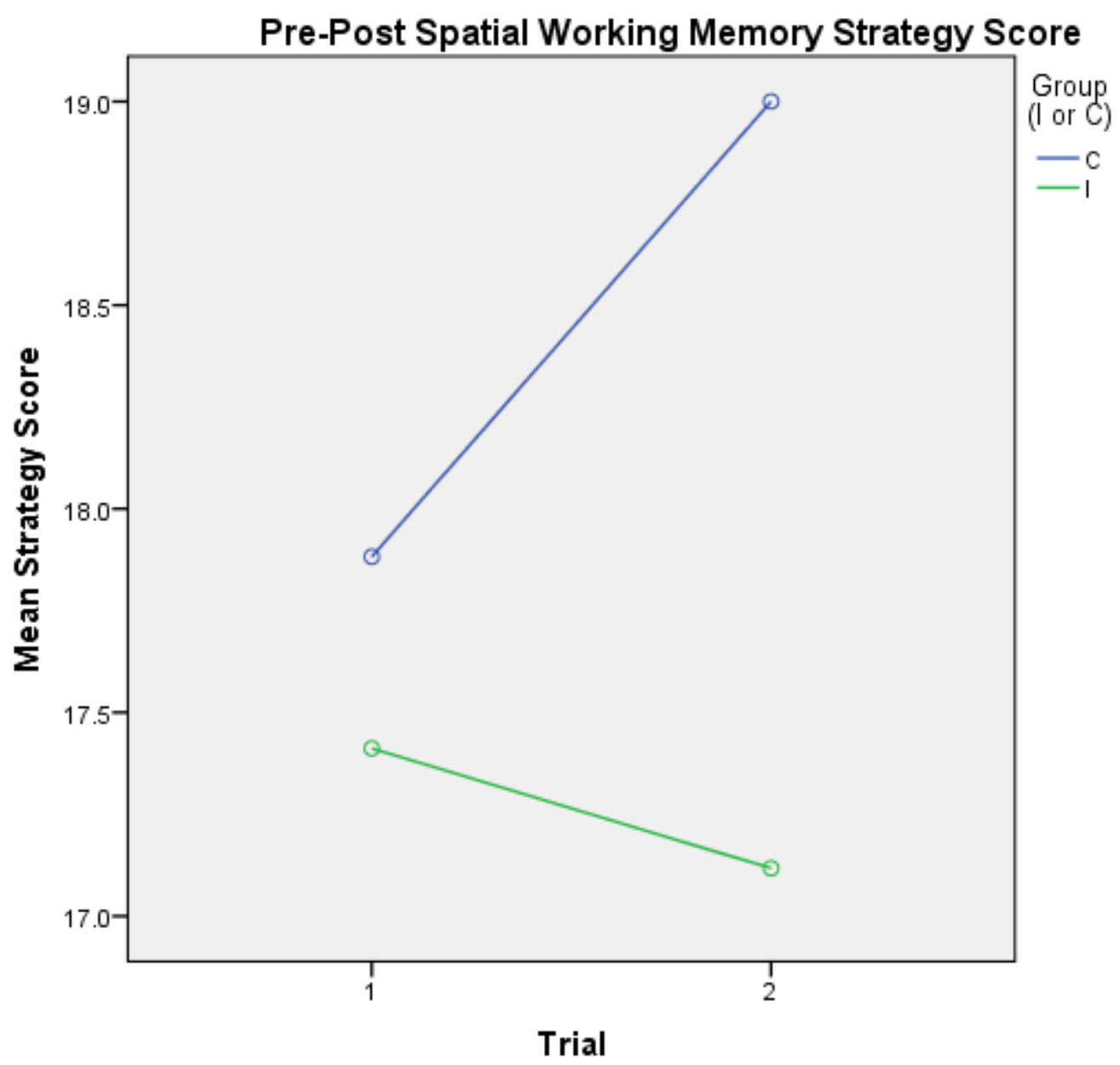

The between-search errors score is defined as the number of times that a participant revisits a box where a token has already been discovered and is calculated for all assessed trials. The control and intervention groups had corresponding mean scores of $17.59(\mathrm{SD}=8.26)$ and $19.65(\mathrm{SD}=7.76)$ for the preliminary assessment and 19.76 $(\mathrm{SD}=9.92)$ and $14.65(\mathrm{SD}=8.58)$ for the post-test. The interaction between intervention and control groups over time were found 
to be statistically significant at a moderate power level $[\mathrm{F}(1,32)=6.759, \mathrm{p}=0.014, \mathrm{ES}=0.174]$ showing a reduction in between-search errors made by the intervention group from pre- to posttest (Figure 4.2).

Figure 4.2: Pre-Post Spatial Working Memory between Error Scores

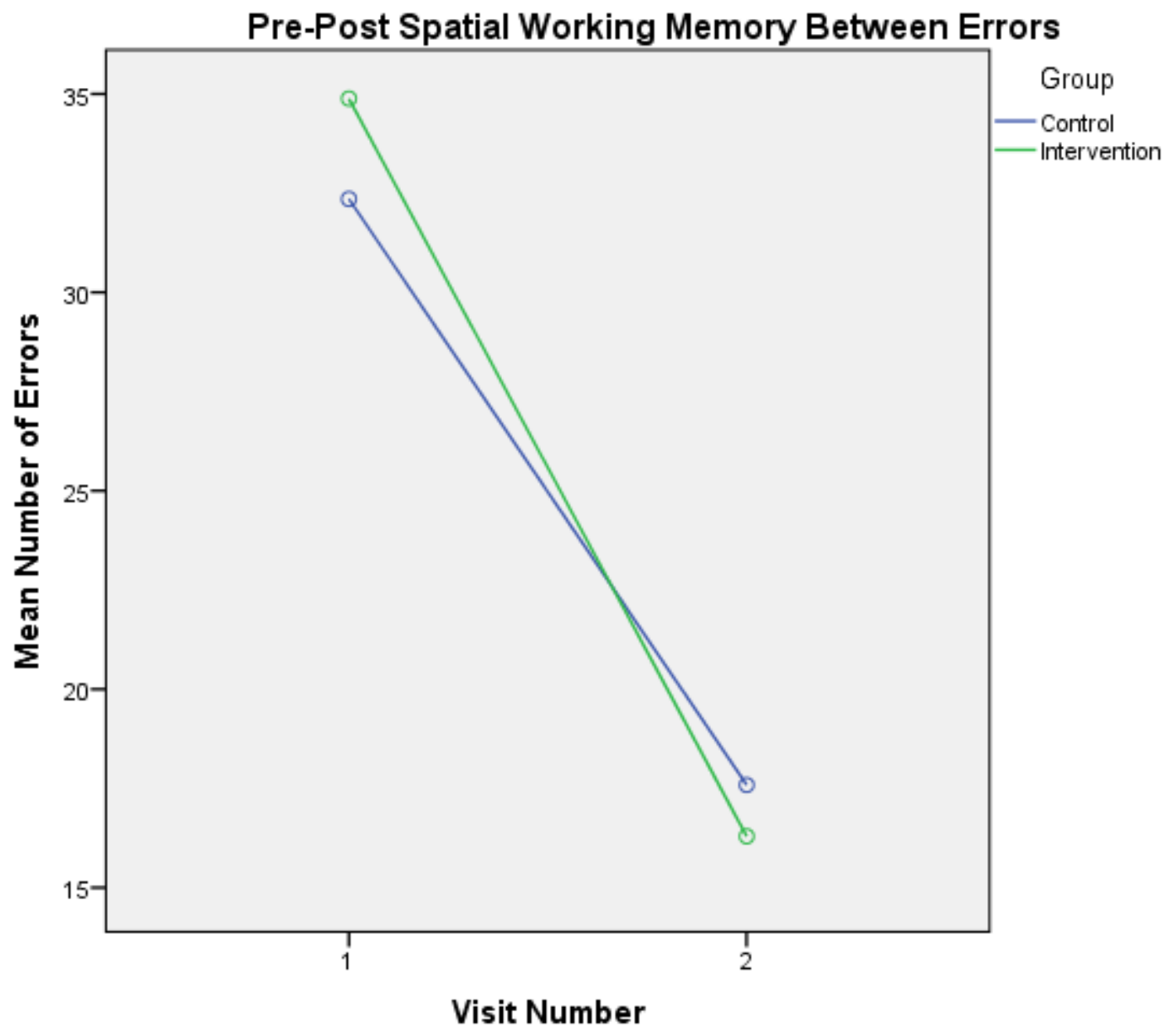

Spatial working memory has been noted to show declines in aging adults at twice the rate of some other contingencies of executive function (Elliott et al., 2011; Myerson J, Emery L, White DA, \& Hale S, 2003). Due to this acknowledged differentiation in memory loss, slight positive changes or maintenance of spatial working memory may be of clinical importance to the aging population. The fact that both SWM outcomes showed positive trends in the intervention 
group, one being statistically significant, may denote that exercise distinctively improves higherlevel cognitive function necessary for more complicated tasks. It may also lead to the belief that the intervention assisted with maintenance and improvement in planning behaviors, goal selection, and execution (Badcock, Michiel, \& Rock, 2005). This outcome could potentially provide important cross-over effects into activities of daily living with particular emphasis on movement safety and efficiency.

Table 4.6: Mean Values for Spatial Working Memory Outcomes

\begin{tabular}{lcccccccc}
\hline \multicolumn{3}{c}{ Control Group } & \multicolumn{5}{c}{ Intervention Group } \\
& & & \multicolumn{3}{c}{ Pre } & & \\
& Pre & & Post & \multicolumn{3}{c}{ Post } \\
Strategy Score & 17.88 & $\mathrm{SD}=2.26$ & 19.00 & $\mathrm{SD}=2.18$ & 17.41 & $\mathrm{SD}=3.45$ & 17.12 & $\mathrm{SD}=3.26$ \\
Between Errors & 17.59 & $\mathrm{SD}=8.26$ & 19.76 & $\mathrm{SD}=9.92$ & 19.65 & $\mathrm{SD}=7.76$ & 14.65 & $\mathrm{SD}=8.58$ \\
\hline
\end{tabular}

Attention Switching Task (AST). During the attention switching task (AST) three values were obtained: (1) congruency cost, (2) switching cost, and (3) percent correct trials. Congruency cost is the difference between response latency (the amount of time from when the stimulus was presented to the moment that the participant pressed the button) of congruent versus incongruent assessed trials. A positive or negative score is obtained for this parameter; a positive score indicates a faster response from the participant for congruent trials while a negative score represents a faster response for incongruent trials. There were no statistically significant interaction between intervention and control groups over time for this assessment [F $(1,32)=0.003, \mathrm{p}=0.956, \mathrm{ES}=0.000]$.

Switching cost is the variance between the response latency of non-switched versus switched assessed trials. For this parameter, a positive score indicates a faster response from the participant for non-switched trials while a negative score represents a faster response for 
switched trials. There were no statistically significant interaction between intervention and control groups over time $[\mathrm{F}(1,32)=0.420, \mathrm{p}=0.521, \mathrm{ES}=0.013]$ for this assessment parameter.

Attention switching task percent of correct trials signifies the percentage of trials in which the participant selected the correct response (mean values displayed in table 4.7). The only statistically significant interaction noted for this testing parameter was the effect of time [F $(1,32)=5.848, \mathrm{p}=0.021, \mathrm{ES}=0.155$ ] denoting a learning effect for this assessment. Table 4.7: Mean Values for Attention Switching Task Outcomes

\begin{tabular}{|c|c|c|c|c|c|c|c|c|}
\hline & \multicolumn{4}{|c|}{ Control Group } & \multicolumn{4}{|c|}{ Intervention Group } \\
\hline & Pre & & Post & & Pre & & Post & \\
\hline \multirow{2}{*}{$\begin{array}{l}\text { Congruency } \\
\text { Cost }\end{array}$} & $166.65 \mathrm{~s}$ & $\mathrm{SD}=$ & $135.09 \mathrm{~s}$ & $\mathrm{SD}=$ & $188.75 \mathrm{~s}$ & $\mathrm{SD}=$ & $155.00 \mathrm{~s}$ & $\mathrm{SD}=$ \\
\hline & & 94.66 & & 98.78 & & 99.70 & & 93.59 \\
\hline \multirow{2}{*}{$\begin{array}{l}\text { Switching } \\
\text { Cost }\end{array}$} & $-167.82 \mathrm{~s}$ & $\mathrm{SD}=$ & -143.94 & $\mathrm{SD}=$ & -106.65 & $\mathrm{SD}=$ & -171.00 & $\mathrm{SD}=$ \\
\hline & & 136.57 & & 114.57 & & 153.21 & & 122.06 \\
\hline $\begin{array}{l}\text { Percent of } \\
\text { Correct } \\
\text { Trials }\end{array}$ & $86.10 \%$ & $\begin{array}{l}\mathrm{SD}= \\
8.26\end{array}$ & $89.85 \%$ & $\begin{array}{l}\mathrm{SD}= \\
9.48\end{array}$ & $89.15 \%$ & $\mathrm{SD}=7.91$ & $91.54 \%$ & $\begin{array}{l}\mathrm{SD}= \\
8.59\end{array}$ \\
\hline
\end{tabular}

Attention switching tasks can be described as utilizing executive control to delineate an appropriate action for the presented stimuli. Though these groups only showed time effects from pre- to post-test indicating an assessment tool training effect, this parameter is of growing importance in relation to the aging population. A litany of studies have been conducted linking attentional demands and fall risk pertaining to posture, allocation of attention, and inability to multitask (Shumway-Cook \& Woollacott, 2000; Lajoie, Teasdale, Bard, \& Fleury, 1993; Lajoie, Teasdale, Bard, \& Fleury, 1996; Brown, Shumway-Cook, \& Woollacott, 1999; Woollacott \& 
Shumway-Cook, 2002). Thus, if attention switching can be learned over time then this can be defined as a potentially valuable finding even if the outcomes were not enriched by the intervention.

Reaction Time (RTI). The final assessment in the executive function battery was the reaction time (RTI) test. RTI was measured via 5-choice reaction time and 5-choice movement time. Five-choice reaction time denotes the speed with which the participant recognizes the stimulus by releasing the press pad button. Five-choice movement time is the time taken after releasing the press pad button to touch the corresponding stimulus on the touchscreen. For both parameters, a lower score denoted a superior score. Utilizing both of these parameters allows the researcher to separate out any motor function acceleration or deceleration resulting from speeding or slowing of cognitive function (mean values displayed in table 4.8 below). Table 4.8: Mean Values for Reaction Time

\begin{tabular}{|c|c|c|c|c|c|c|c|c|}
\hline & \multicolumn{4}{|c|}{ Control Group } & \multicolumn{4}{|c|}{ Intervention Group } \\
\hline & Pre & & Post & & Pre & & Post & \\
\hline $\begin{array}{l}\text { 5-Choice } \\
\text { Reaction } \\
\text { Time }\end{array}$ & $391.98 \mathrm{~ms}$ & $\begin{array}{l}\mathrm{SD}= \\
46.80\end{array}$ & $385.44 \mathrm{~ms}$ & $\begin{array}{l}\mathrm{SD}= \\
54.22\end{array}$ & $379.30 \mathrm{~ms}$ & $\begin{array}{l}\mathrm{SD}= \\
76.63\end{array}$ & $374.16 \mathrm{~ms}$ & $\begin{array}{l}S D= \\
67.96\end{array}$ \\
\hline $\begin{array}{l}\text { 5-Choice } \\
\text { Movement } \\
\text { Time }\end{array}$ & $500.95 \mathrm{~ms}$ & $\begin{array}{l}\mathrm{SD}= \\
69.45\end{array}$ & $530.72 \mathrm{~ms}$ & $\begin{array}{l}\mathrm{SD}= \\
95.54\end{array}$ & $466.33 \mathrm{~ms}$ & $\begin{array}{l}\mathrm{SD}= \\
87.67\end{array}$ & $471.46 \mathrm{~ms}$ & $\begin{array}{l}\mathrm{SD}= \\
85.13\end{array}$ \\
\hline
\end{tabular}

The literature supports that older adults adopt a more conservative response due to a nondecisional component of processing paired with slowed retrieval of perceptual information (Ratcliff, Thapar, \& McKoon, 2006b; Ratcliff, Thapar, \& McKoon, 2006a). Despite supporting literature and the proclaimed ability for this assessment to detect small changes in reaction time, both the RTI 5-choice reaction time $[\mathrm{F}(1,32)=0.012, \mathrm{p}=0.912, \mathrm{ES}=0.00]$ and the RTI 5- 
choice movement time $[\mathrm{F}(1,32)=1.069, \mathrm{p}=0.309, \mathrm{ES}=0.032]$ results showed no statistical interaction between intervention and control groups over time. This could be due to technical errors that were experienced during the final assessment period. Many participants experienced a response capability slowing of the program, meaning that when they pushed the button on the screen, it took more time for the program to respond to their touch. This error was addressed but not able to be fully remedied for the post-testing sessions. This may have led to the insignificant RTI results.

Qualitative Findings. Though the focus group was not originally intended to assist in answering RQ1, the researcher found it prudent to include commentary pertaining to cognitive function that emerged from the focus group. Remarks relating directly to cognitive function were not high in frequency (13 comments in total), nor were they well distributed amongst the participants (4 of the 13 comments were from a single respondent). Three categories emerged pertaining to RQ1 upon inductive analysis of the transcripts: (1) Proactivism, (2) Alertness, and (3) Improved Movement Quality.

Motivation to promote and maintain mental acuity was the most pervasive collection of comments pertaining to cognitive function. Concern of losing executive abilities is a known fear in the aging population. The CDC reports that "we are twice as fearful of losing our mental capacity as having diminished physical ability and $60 \%$ of adults are very or somewhat worried about memory loss" (cdc.gov, pg 3). This is possibly why the concept of being proactive for cognitive retention emerged. The following example from a focus group participant may be perceived as jovial, but it suggests that cognitive decline is indeed a concern within this group: 
My husband, he suffers from dementia some and I think the reason he pushed me into this was because if I got it too then that would be the end. Between the two of us there is one of us.

Other comments pertaining to loss of mental acuity were embedded in answers for various focus group questions that did not necessarily pertain to cognitive function.

The indigenous typology of "alertness" arose from comments of feeling more alert throughout the course of the day due to participation in aquatic exercise. The researcher also noted that an additional six comments were made relating to energy level and many of the participants were using the words interchangeably or in conjunction with one another.

After further reflection on the transcripts, a third analyst typology of improved movement quality emerged. Improved movement quality, including ability to perform ADLs more effectively, coordination improvements, and balance, was a topic that permeated the focus group commentary. Though these comments may not have been intended to reflect upon cognitive parameters, neuromuscular improvements are well documented in the exercise literature (Radaelli et al., 2013; Taaffe, Duret, Wheeler, \& Marcus, 1999; Brach, Van Swearingen, Perera, Wert, \& Studenski, 2013; Cadore, Rodriguez-Manas, Sinclair, \& Izquierdo, 2013). The role of the nervous system in biomechanics is well noted but rarely a primary focus for intervention strategies. The concept of utilizing repetitive movements to promote motor learning via neurological adaptation has been noted to improve movement control (Brach et al., 2013). One of the primary emergent categories throughout the focus group regardless of moderator-driven questions was that of improved ADLs and function. A component of the participants perceived improvements in physical ability level may have been, in part, due to the movement variety practiced during the aquatic intervention. As one participant states: 
My coordination isn't the best in the world, and I find by exercise or repetition like we did in class that I saw a big difference at the end of the class than the beginning... at the end I felt that I was much better in following.

Motor learning is a form of procedural knowledge, which can be directly linked to executive function. Many parameters of executive function may play a primary role in motor learning processes and improvements thus; this finding could be of clinical relevance for endorsing movement safety in the aging population.

Triangulation for RQ1. Triangulation of the quantitative and qualitative findings revealed a convergence of the data favoring that the intervention did not produce global improvements in executive function (Appendix $\mathrm{J}$ ). The lack of commentary on the topic of cognitive function pared with the predominately statistically insignificant quantitative findings promotes the belief that there were no noteworthy changes observed in global executive function. In contrast, comparison of these research methods suggest that some neurological changes to promote movement economy were manifest as noted in the qualitative findings aligning the positive trends ( $p=0.152$ for "strategy") and statistical significance ( $p=0.014$ for "between errors") seen in the SWM assessment. Improvements in SWM are attributed to maintenance and improvement in planning behaviors, goal selection, and execution (Badcock et al., 2005).

Triangulation promotes the conclusion that the aquatic intervention led to cross-over effects into activities of daily living with particular emphasis on movement safety and efficiency.

\section{Research Question Two}

$R Q$ 2: Does participation in a 10-week aquatic exercise class result in a statistically significant $(p \leq .05)$ improvement in cardiovascular fitness when utilizing the Kruel target heart rate deduction to configure individualized target heart rate zones when compared to the control group? 
Cardiovascular fitness was primarily assessed by the 6-minute walk test, though resting heart rate and blood pressure were also taken into account when evaluating changes in fitness. Physical inactivity, RHR variability, as well as resting SBP and DBP have been associated with reduced cardiovascular health (Farrell, 2011). Due to the impending link of RHR, SBP, and DBP to cardiovascular health, these variables were also assessed at baseline and at the conclusion of the intervention.

Six-Minute Walk Test. Mean values for both the intervention and control group on the 6-minute walk test were within normal limits prior to and following the intervention period (Table 4.1). A 2-way ANOVA was used to compare pre- and post-test mean distances achieved by the participants. Total walking distance covered (in meters) during 6 minutes was compared after the 10-week intervention to reveal that the control and intervention groups displayed no statistically significant interaction between intervention and control groups over time $\mathrm{F}(1,31)=$ $1.907, \mathrm{p}=0.177, \mathrm{ES}=0.058$.

As illustrated in figure 4.3, a slight improvement in the intervention group and small reduction in distance walked (meters) in the control group was observed; the small sample size and associated power of this study may be the reason for why the difference in the 6-minute walk test did not reach statistical significance. Research indicates that a mean change of 54 meters is associated with clinical significance (Redelmeier, Bayoumi, Goldstein, \& Guyatt, 1997). Although the intervention group only achieved a positive mean change of $20.77 \mathrm{~m}(\mathrm{SD}=73.64)$, the control group's reduction of $29.01 \mathrm{~m}(\mathrm{SD}=5.61)$ within a 10 -week period could hold great clinical significance when compounded over a year's duration of sedentary behavior. Current studies support an enhanced predictability for the 6-minute walk distance achieved (Steffens et al., 2013). The compounding factors experienced with aging —reduced skeletal, muscle mass, 
and strength — in combination with lower cardiorespiratory function commonly results in an impaired functional status. The 10-week intervention may not have stimulated a strong enough physiologic response to result in a statistically significant post-intervention 6-minute walk test, however, all participants either maintained or improved upon their prior walking assessment while the control group experienced a reduction in performance. The aquatic exercise sessions may have provided enough physical activity to hinder the effects of aging resulting in improved performance relative to their baseline values in the 6-minute walk test.

Figure 4.3: Pre-Post Scores for Distance Walked in meters

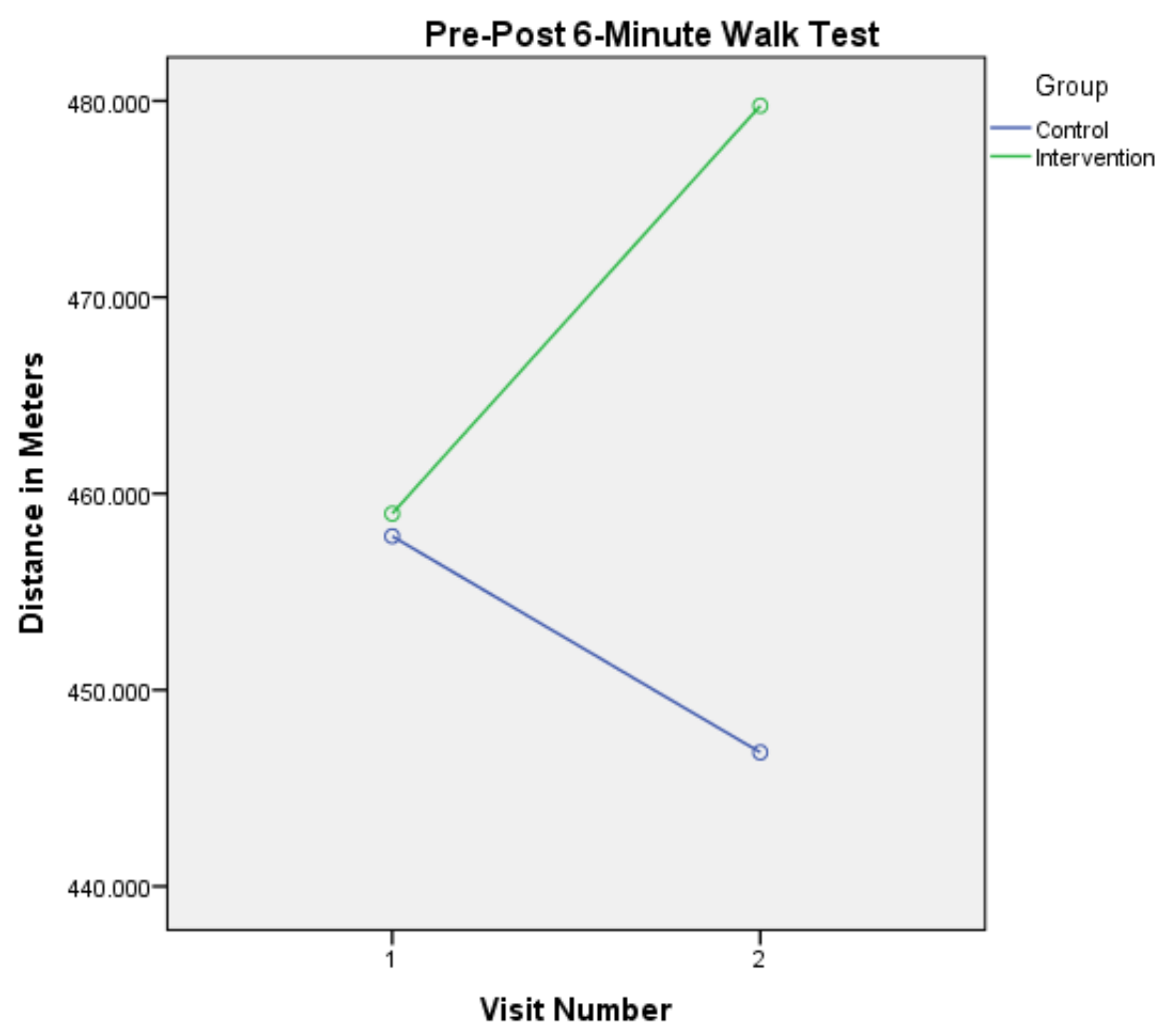

RHR, SBP, DBP. Mean values for RHR, SBP, and DBP are displayed in Table 4.9 below and show that there was no statistically significant interaction between intervention and control groups over time for $\operatorname{RHR}[\mathrm{F}(1,32)=0.041, \mathrm{p}=0.841, \mathrm{ES}=0.001]$ or $\operatorname{SBP}[\mathrm{F}(1,32)=$ 0.553, $\mathrm{p}=0.462, \mathrm{ES}=0.017]$. Resting DBP, however, was statistically significant $[\mathrm{F}(1,32)=$ 
$6.699, \mathrm{p}=0.014, \mathrm{ES}=0.173]$. As illustrated by figure 4.4, DBP was substantially reduced relative to baseline after 10 weeks of aquatic exercise.

Figure 4.4: Pre-Post Scores for Diastolic Blood Pressure

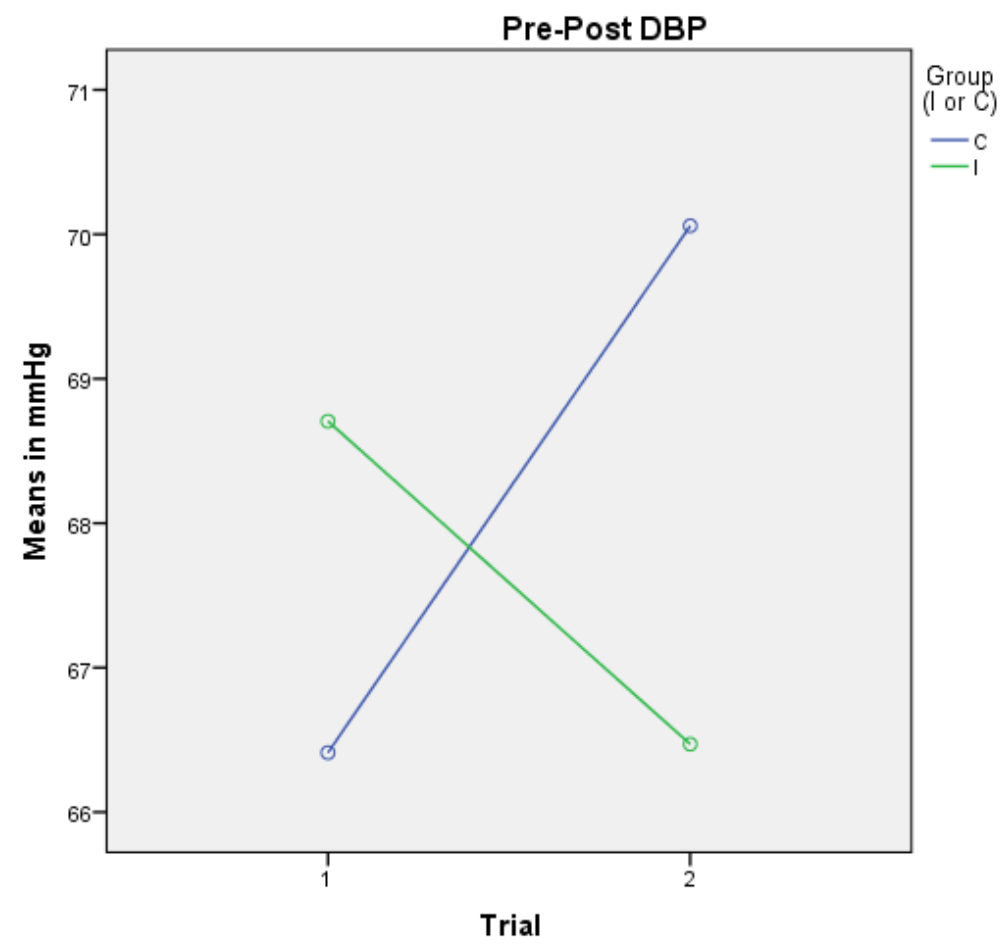

The SBP and DBP showed positive trends for the intervention group and negative trends in the control group, but the within group effects for SBP did not reach a level of statistical significance. This may have been due to medications, exercise intensity adherence, or the fact that pre-post measurements were taken during different times of the day. Medications can stabilize these cardiovascular factors thereby reducing the variability during both rest and exercise. Moderate exercise intensity, defined as 50-70\% of maximal heart rate, is noted to reduce RHR by 5-20 bpm in healthy adults; however, the introduction of some medications can alter this response. Exercise intensity was gauged with heart rate and correction factors were imparted for both the effects of immersion as well as use of specified medications. Even with the introduction of these correction factors, some participants did not maintain the 
individualized, pre-determined intensity levels. Lack of maintaining a moderate intensity level may have resulted in a reduction of positive physiologic effect pertaining to cardiovascular factors. As illustrated by figure 4.5, the percentage of time spent in the participant's individualized THR zone gradually reduced as the intervention became more challenging and required a higher THR. However, figure 4.6 displays that the participants average RPE for the classes gradually increases. ACSM supports the use of using RPE in addition to HR when monitoring exercise intensity to allow for greater precision (Farrell, 2011). Some literature supports the use of rate of perceived exertion (RPE) as an effective aid in determining intensity for those on HR-regulating medications, such as beta-blockers. Assessing both THR and RPE variables allow for a greater understanding of the participants objective and subjective physiological response to the intervention. As evident from figure 4.5 and 4.6, intervention participants were not able to maintain their THR but did meet the ASCM recommendations outlined for RPE during the 10-week intervention.

Figure 4.5: Percentage of Time spent in Target Heart Rate Zone

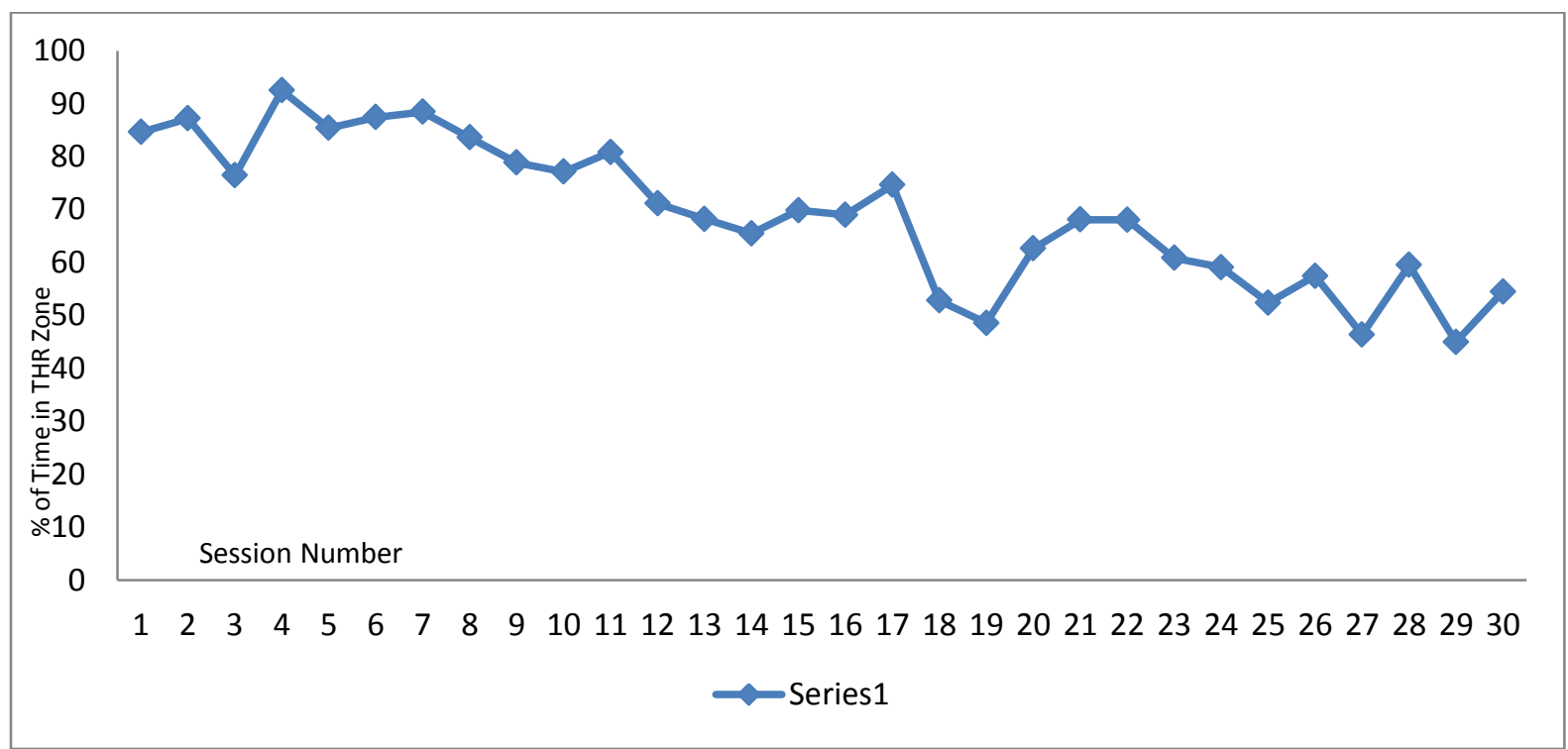


Figure 4.6: Average Rating of Perceived Exertion

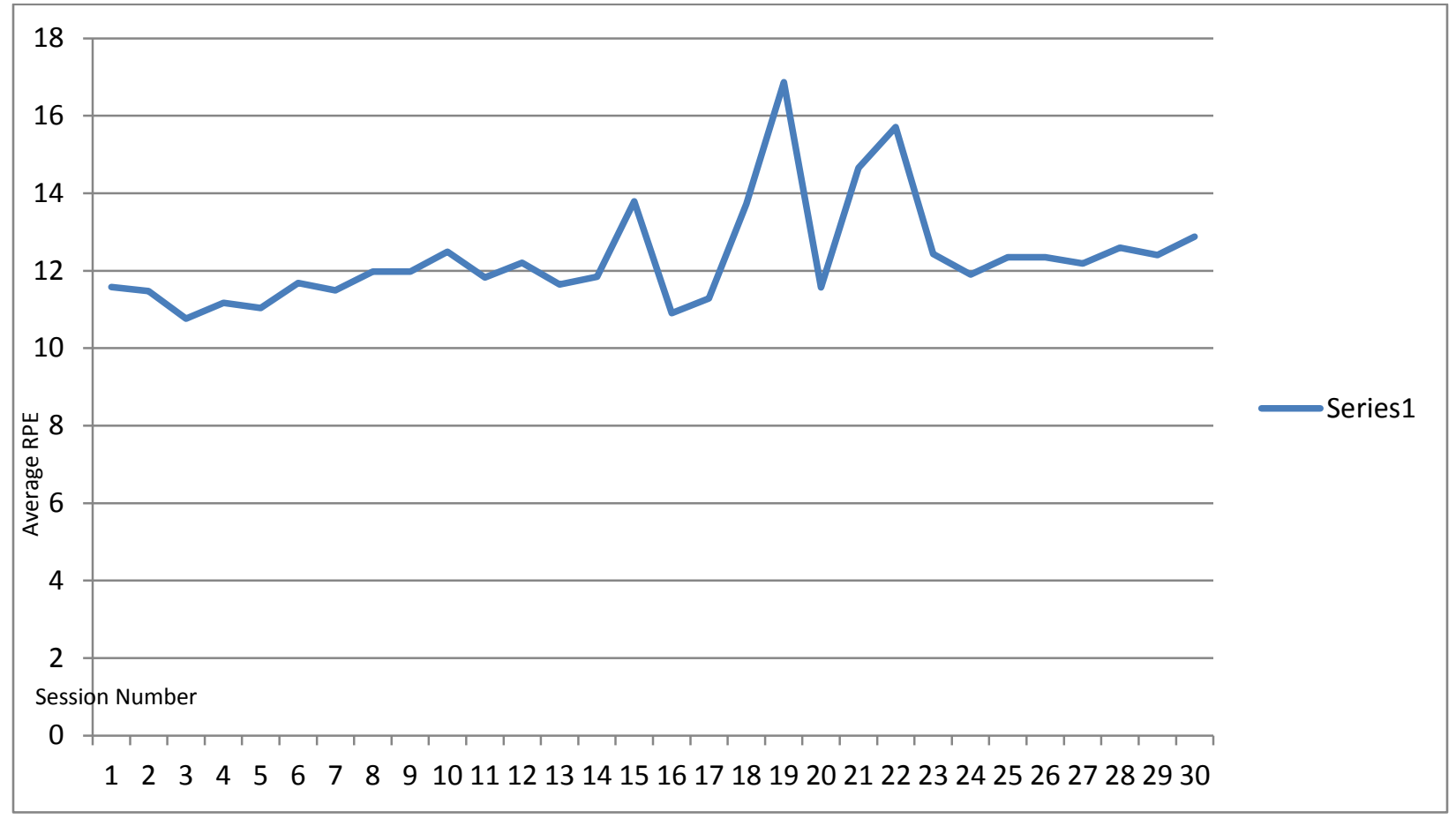

The final variation that may have confounded the outcomes of these variables is testing time. Time of test was arranged in accordance with the participant's individual schedules and thus was not necessarily the same for pre- and post-test. Resting HR and BP includes diurnal rhythmic and nonrhythmic fluctuations that occur due to alterations of homeostatic needs that vary throughout the course of the day. 
Figure 4.7 Pre-Post Systolic Blood Pressure

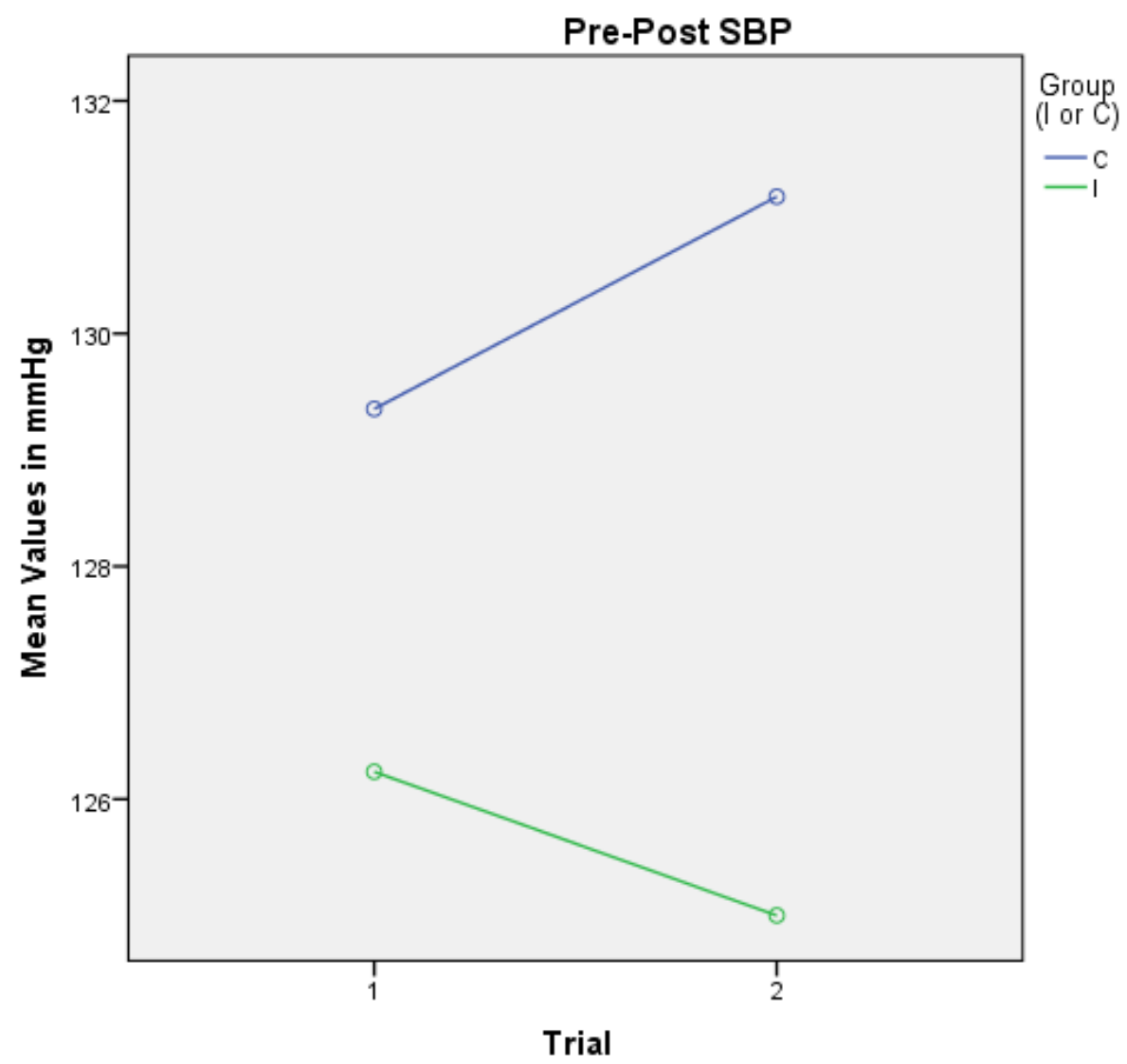

Table 4.9: Mean Values for Cardiovascular Fitness Variables

\begin{tabular}{|c|c|c|c|c|c|c|c|c|}
\hline & \multicolumn{4}{|c|}{ Control Group } & \multicolumn{4}{|c|}{ Intervention Group } \\
\hline & Pre & & Post & & Pre & & Post & \\
\hline $\begin{array}{l}6 \text { Minute Walk } \\
\text { Test Distance } \\
(\text { norm }=405- \\
498 \mathrm{~m})\end{array}$ & $457.83 \mathrm{~m}$ & $\begin{array}{l}\mathrm{SD}= \\
74.10\end{array}$ & $446.82 \mathrm{~m}$ & $\begin{array}{l}\mathrm{SD}= \\
84.9\end{array}$ & $458.98 \mathrm{~m}$ & $\begin{array}{l}\mathrm{SD}= \\
106.35\end{array}$ & $479.75 \mathrm{~m}$ & $\begin{array}{l}\mathrm{SD}= \\
119.48\end{array}$ \\
\hline $\begin{array}{l}\text { Resting Heart } \\
\text { Rate }\end{array}$ & $\begin{array}{l}62.12 \\
\mathrm{bpm}\end{array}$ & $\begin{array}{l}\mathrm{SD}= \\
12.84\end{array}$ & $\begin{array}{l}69.29 \\
\text { bpm }\end{array}$ & $\begin{array}{l}\mathrm{SD}= \\
10.45\end{array}$ & $63.71 \mathrm{bpm}$ & $\begin{array}{l}\mathrm{SD}= \\
7.24\end{array}$ & $\begin{array}{l}62.29 \\
\mathrm{bpm}\end{array}$ & $\mathrm{SD}=9.19$ \\
\hline $\begin{array}{l}\text { Resting } \\
\text { Systolic Blood } \\
\text { Pressure }\end{array}$ & $\begin{array}{l}130.00 \\
\mathrm{mmHg}\end{array}$ & $\begin{array}{l}\mathrm{SD}= \\
23.64\end{array}$ & $\begin{array}{l}132.65 \\
\mathrm{mmHg}\end{array}$ & $\begin{array}{l}\mathrm{SD}= \\
17.89\end{array}$ & $\begin{array}{l}128.71 \\
\mathrm{mmHg}\end{array}$ & $\begin{array}{l}\mathrm{SD}= \\
18.96\end{array}$ & $\begin{array}{l}126.53 \\
\mathrm{mmHg}\end{array}$ & $\begin{array}{l}\mathrm{SD}= \\
14.84\end{array}$ \\
\hline $\begin{array}{l}\text { Resting } \\
\text { Diastolic Blood } \\
\text { Pressure }\end{array}$ & $\begin{array}{l}66.18 \\
\mathrm{mmHg}\end{array}$ & $\begin{array}{l}\mathrm{SD}= \\
11.33\end{array}$ & $\begin{array}{l}70.29 \\
\mathrm{mmHg}\end{array}$ & $\begin{array}{l}\mathrm{SD}= \\
12.89\end{array}$ & $\begin{array}{l}69.29 \\
\mathrm{mmHg}\end{array}$ & $\begin{array}{l}\mathrm{SD}= \\
9.59\end{array}$ & $\begin{array}{l}68.71 \\
\mathrm{mmHg}\end{array}$ & $\begin{array}{l}\mathrm{SD}= \\
10.68\end{array}$ \\
\hline
\end{tabular}


Though these values do not entirely support the hypothesis that a 10-week aquatic exercise program will result in statistically significant improvements in cardiovascular parameters, the general trends of the parameters assessed have positive implications for the intervention group. The aquatic exercise sessions may have provided enough physical activity to hinder the effects of aging resulting in improved performance relative to their baseline values in the 6-minute walk test and SBP. With the exception of DBP, the null hypothesis that the intervention does not improve cardiovascular fitness is accepted.

\section{Research Question Three}

RQ3: Do statistically significant ( $p \leq .05)$ positive changes occur for general fitness and wellness parameters after participation in a 10-week aquatic exercise class when compared to the control group?

Anthropometric measurements including weight and body composition were taken along with resting blood pressure, and resting heart rate pre and post intervention. As discussed previously, no statistically significant pre to post changes were noted for RHR or resting SBP; however, a statistical significant reduction was realized for resting DBP.

Weight. Weight in pounds and body composition were also wellness parameters assessed for effect. Weight is a variable that cannot appropriately be compared to norm charts when referring to averages, though positive changes in weight (for this sample, a reduction in weight) have been noted to reflect progressive advancement towards better overall health status. The findings of this study for the dependent variable of weight in pounds (Table 4.10) showed no statistically significant main effects (time or group) or interactions, and all effects were small. The trending pattern for the intervention group was a downward pattern, though as stated previously, it was not a statistically significant interaction between intervention and control groups over time, finding $[\mathrm{F}(1,32)=1.161, \mathrm{p}=0.289, \mathrm{ES}=0.035]$. It is well documented that 
long term global health benefits can be observed with attainment of a modest 5-10\% weight loss (Goldstein, 1992; Vidal, 2002). Some of the primary advances noted due to weight loss include improved blood pressure, blood cholesterol, and blood glucose control, overall cardiovascular health, and improved mobility (Vidal, 2002; Goldstein, 1992). .

Body Composition. There also was no statistically significant interaction between intervention and control groups over time $[\mathrm{F}(1,32)=0.346, \mathrm{p}=0.560, \mathrm{~F}=0.11]$ in mean percent body fat (Table 4.10) in the intervention group. Intimately related to weight, positive alterations in body composition can provide similar health outcomes (Vidal, 2002; Goldstein, 1992; Farrell, 2011). The aging process is notoriously related to advanced muscle wasting, otherwise known as sarcopenia, in addition to increased obesity levels. The relation of weight-determined obesity to body composition is not always linear and this is particularly evident in the aging population. Studies have shown that sarcopenia increases from $15 \%$ at ages $60-69$ years to $40 \%$ in individuals over the age of 80 years regardless of body fat (Baumgartner et al., 2004). It is also noted that a progressive loss of $3-5 \%$ of total muscle mass per decade of life resulting in reductions in strength and quality of life is well established (Nair, 1995). Thus, it is more imperative to show maintenance or improvements in lean body mass (muscle, bone, connective tissues) in this cohort than weight loss.

Table 4.10: Mean Values of Wellness Parameters of Body Weight and Percent Body Fat

\begin{tabular}{|c|c|c|c|c|c|c|c|c|}
\hline & \multicolumn{4}{|c|}{ Control Group } & \multicolumn{4}{|c|}{ Intervention Group } \\
\hline & Pre & & Post & & Pre & & Post & \\
\hline Body Weight & $195.65 \mathrm{lbs}$ & $\mathrm{SD}=59.47$ & $195.37 \mathrm{lbs}$ & $\begin{array}{l}\mathrm{SD}= \\
58.60\end{array}$ & $185.94 \mathrm{lbs}$ & $\begin{array}{l}\mathrm{SD}= \\
45.74\end{array}$ & $184.241 \mathrm{bs}$ & $\begin{array}{l}\mathrm{SD}= \\
45.71\end{array}$ \\
\hline $\begin{array}{l}\text { Body } \\
\text { Composition }\end{array}$ & $40.78 \%$ & $\mathrm{SD}=7.71$ & $42.47 \%$ & $\mathrm{SD}=8.15$ & $40.67 \%$ & $\begin{array}{l}\mathrm{SD}= \\
7.64\end{array}$ & $41.69 \%$ & $\mathrm{SD}=8.18$ \\
\hline
\end{tabular}


Global Wellness. Wellness, wellbeing, and quality of life are used synonymously when referencing an individual's health-related welfare. Though they are not the same, this bond between the aforementioned factors is tightly intertwined. When improved, wellness parameters, including the ones assessed, have been shown to clearly advance quality of life. In a different lens, when wellness and quality of life are impaired, so too is wellbeing. Quality of life and wellbeing are both subjective and self-reported while wellness can be measured more objectively.

In the case of this study, the objectively measured wellness parameters-RHR, SBP, weight, and body composition—showed no statistically significant improvement while DBP did reach statistical significance after the 10 -week intervention. These findings mirror results from other aquatic-exercise based studies with similar intervention programs and populations (Rica et al., 2013). However, qualitative reports of improved quality of life and wellbeing were recurrently reported throughout the focus group transcripts.

During the two focus groups, participants reported improvements in endurance, ease of movement, alertness and energy level, flexibility, balance, pain levels, muscular strength and definition, activities of daily living, reduction in need for assistive devices, recovery of physical abilities, mood and emotion, as well as coordination. As Tina stated "I noticed a difference on the stairs for me. I can actually get to the top and don't have to stop and can breathe now. There is a difference." Perceptions of positive alterations in quality of life were described by $100 \%$ of the focus group participants, many with multiple elocutions professing gains in well-being. Phrases such as "I see the difference," "I feel good about myself," and "I have more energy" reinforce the overall belief in positive changes within their global wellness. The analyst typology of improved global wellness was heavily displayed through frequency analysis within 
the transcriptions: 15 comments about improving activities of daily living, 11 remarks acknowledging improved movement and balance, 2 remarks concerning ability to reduce reliance on assistive devices for ambulation, and 9 statements concerning improved energy were distinguished.

Though the focus group remarks may be regarded as perceptual and not statistical in nature, the power of perception has been a well-documented phenomenon resulting in subsequent health outcomes (Fylkesnes \& Forde, 1992; Fylkesnes \& Forde, 1991; Foottit \& Anderson, 2012). Perceptions concerning health status have been reported to influence health behaviors including preventative screening, engagement in good self-care practices, or adherence to health-related programming (Idler, Leventhal, McLaughlin, \& Leventhal, 2004; Idler \& Benyamini, 1997; Foottit \& Anderson, 2012). Thus it could be presumed that the positive perception of improved wellness, wellbeing, and quality life described by the intervention group could lead to health-improving behaviors.

Triangulation for RQ2 and RQ3. The quantitative and qualitative findings addressing wellness (RQ2 and RQ3) were converged onto a matrix to offer a more complete understanding of the identified parameter (Appendix K). Of the quantitative values, only resting DBP was found to show statistical significance $(\mathrm{p}=0.014)$ though 35 separate statements were made by the focus group participants, all of which contributed, pertaining to improvements in global wellness and overall improvements in quality of life. The quantitative findings and related commentary show some convergence and some divergence. Distance walked, resting SBP and DBP quantitatively presented with positive trends. Though BP is commonly asymptomatic in nature, walking ability is readily observable. During the focus groups, 6 walking-specific comments and 24 walking-related (endurance, balance, coordination, etc.) comments were made. 
The entirety of the group, $100 \%$ of the participants, contributed to the aforementioned commentary, supplying at least one of the comments being appraised. All 30 comments were associated with positive improvements in ability level, with no negative comments being made. The absence of negative comments either reinforces that the positive quantitative trend observed is clinically relevant, or it may identify response bias. These findings also promote the impression that sample size was too small to assert the appropriate statistical power for the quantitative analysis.

Comments on physical ability were plentiful (35 total), however, comments on weight and body composition were missing from the focus group commentary. Only 3 remarks were made directly pertaining to weight and body composition, 2 positive and 1 negative. This lack of commentary concerning this topic converges with the quantitative data showing no statically significant changes to these parameters.

\section{Research Question Four}

RQ4: Does participation in a 10-week aquatic exercise class result in a statistically significant $(p \leq .05)$ alteration in social support, self-efficacy, motivators, or exercise barriers when compared to the control group?

Participants in this study took part in 3 surveys pertaining to exercise barriers (e.g., fatigue, time, pain), social support, self-efficacy, and motivators (e.g., reducing pain, diminishing illness, advice from doctor). The intervention and control groups completed the surveys (Appendix F) during their pre and post-testing while the intervention group also completed a mid-intervention survey $(n=34)$. Calculation of barriers proceeded by adding all 13 listed barriers and averaging them. The highest rating for any item was 5, and accordingly, the highest mean possible was 5. Similar calculations were made for motivators (6 items) where the highest score was 6 , thus the highest mean possible was 6 , and barriers to self-efficacy (10 items) the 
highest score of 5 and highest possible mean value of 5 . The values for social support were given numerical codes relating to the participants choices: $1=$ not at all, $2=$ very little, $3=$ somewhat, and $4=$ a lot. The question concerning self-efficacy was coded in a similar fashion with $1=$ very unsure, $2=$ somewhat unsure, $3=$ somewhat sure, and $4=$ very sure. All values are listed in Table 4.11 below.

Table 4.11: Mean Values for Psychosocial Mediator Variables

\begin{tabular}{|c|c|c|c|c|c|c|c|c|c|c|}
\hline & \multicolumn{4}{|c|}{ Control Group } & \multicolumn{6}{|c|}{ Intervention Group } \\
\hline & Pre & & Post & & Pre & & Mid & & Post & \\
\hline $\begin{array}{l}\text { Total } \\
\text { Barriers* }\end{array}$ & 1.94 & $\begin{array}{l}\mathrm{SD}= \\
0.46\end{array}$ & 1.85 & $\begin{array}{l}\mathrm{SD}= \\
0.37\end{array}$ & 2.11 & $\begin{array}{l}\mathrm{SD}= \\
0.59\end{array}$ & 2.13 & $\begin{array}{l}\mathrm{SD}= \\
0.55\end{array}$ & 2.09 & $\begin{array}{l}\mathrm{SD}= \\
0.38\end{array}$ \\
\hline Motives** & 3.98 & $\begin{array}{l}\mathrm{SD}= \\
1.55\end{array}$ & 3.69 & $\begin{array}{l}\mathrm{SD}= \\
1.44\end{array}$ & 4.60 & $\begin{array}{l}\mathrm{SD}= \\
1.11\end{array}$ & 4.54 & $\begin{array}{l}\mathrm{SD}= \\
0.81\end{array}$ & 4.57 & $\begin{array}{l}\mathrm{SD}= \\
0.87\end{array}$ \\
\hline $\begin{array}{l}\text { Barriers to } \\
\text { Self- } \\
\text { Efficacy*** }\end{array}$ & 3.96 & $\begin{array}{l}\mathrm{SD}= \\
0.84\end{array}$ & 3.53 & $\begin{array}{l}\mathrm{SD}= \\
0.74\end{array}$ & 3.82 & $\begin{array}{l}\mathrm{SD}= \\
0.72\end{array}$ & 3.61 & $\begin{array}{l}\mathrm{SD}= \\
0.50\end{array}$ & 3.68 & $\begin{array}{l}\mathrm{SD}= \\
0.75\end{array}$ \\
\hline $\begin{array}{l}\text { Social } \\
\text { Support**** }\end{array}$ & 3.21 & $\begin{array}{l}\mathrm{SD}= \\
0.61\end{array}$ & 2.94 & $\begin{array}{l}\mathrm{SD}= \\
0.78\end{array}$ & 3.47 & $\begin{array}{l}\mathrm{SD}= \\
0.54\end{array}$ & 3.59 & $\begin{array}{l}\mathrm{SD}= \\
0.87\end{array}$ & 3.47 & $\begin{array}{l}\mathrm{SD}= \\
0.54\end{array}$ \\
\hline $\begin{array}{l}\text { Self- } \\
\text { Efficacy***** }\end{array}$ & 3.84 & $\begin{array}{l}\mathrm{SD}= \\
0.76\end{array}$ & 3.58 & $\begin{array}{l}\mathrm{SD}= \\
0.75\end{array}$ & 3.82 & $\begin{array}{l}\mathrm{SD}= \\
0.72\end{array}$ & 3.06 & $\begin{array}{l}\mathrm{SD}= \\
0.66\end{array}$ & 3.68 & $\begin{array}{l}\mathrm{SD}= \\
0.75\end{array}$ \\
\hline
\end{tabular}

Barriers \& Motivators. As noted in the literature, barriers and motivators share great commonality within the aging population. Motivators including deteriorating health, increased time availability, being educated or referred by a physician to exercise, and closer proximity to exercise facilities play the role of both barrier and motivator in the elderly (Cohen-Mansfield et al., 2006; Schutzer \& Graves, 2004; Schutzer \& Graves, 2004). For this reason it is important to discuss barriers and motivators in accordance with one another within this population. 
Preliminary (baseline) measures for perceived barriers for both the control and intervention groups showed that the groups did not identify any strong barriers to exercise participation (mean values listed in Table 4.11). The difference between the intervention and control groups over time was not statistically significant $[\mathrm{F}(1,31)=0.011, \mathrm{p}=0.918, \mathrm{ES}=0.000]$. Due to their affiliation, it is not surprising that motives to exercise also received very positive ratings. Similarly, the findings for differences in motives between the intervention and control groups over time were not statistically significant $[\mathrm{F}(1,31)=1.008, \mathrm{p}=0.323, \mathrm{ES}=0.031]$. The ratings for both barriers and motivators for exercise were initially rated so highly that there was a ceiling effect for the measurement. Post-intervention measurements had very little room for enhancement due to the initial ratings. What the intervention group's post-test did indicate is that after participation in the 10-week exercise program there was not a statistically significant change in barriers or motivators, both remained very positive. This may have positive implications on behavior change, adherence, and continuance of physical activity amongst the intervention participants.

Barriers. Inductive and frequency analyses of the focus group transcripts supported many of the literature-identified barriers including physical limitations, knowledge, fear, and accessibility. Five barrier categories were identified: (1) priorities, (2) accessibility, (3) support, (4) fear of failure, and (5) health status. The most frequently mentioned barrier, noted by $100 \%$ of the focus group attendees, was facility accessibility, namely parking and traffic. Many of the participants reported developing coping strategies to overcome this barrier throughout the course of the study by means of appropriation of time allowance. An excellent example was when Pamela stated that "It was hard to park sometimes. That was a trick to learn. To come early enough to find a parking place or gauge that." Greater time allocation to assure that they would 
arrive to the exercise sessions on time shows the participants commitment to the study and their ability to overcome presented barriers.

Prioritization of aquatic exercise session attendance was further delineated into subcategories of scheduling and other obligations including family. Accessibility, though its own classification, demonstrated how the intervention group would prioritize by reallocating time towards assuring attendance. Likewise, the participants showed resolve in navigating around most of the barriers associated with prioritization. In the case of scheduling, some would attend different exercise class times (morning or afternoon) due to personal scheduling shifts while others would set multiple alarms to assure they were able to awake in time for class. Many of the perceived family-related obligations that the intervention group experienced were not as successfully circumnavigated. In addition focus group attendees also spoke of lack of family support, absence of family's belief in ability, family's needs, scheduled trips, and chores as primary grounds for potential exercise session absence. Though most of these barriers were selfremoved via restructuring of time and exercise modality or location, family needs were commonly prioritized above exercise class attendance establishing the strength of familial support or lack thereof with one participant stating "nothing I could have done about that" in reference to a family-oriented barrier.

Deficiency of support systems was also noted by the participants as an acting barrier for their participation in the exercise sessions. Though most contributors purported a bounty of support systems with comments such as "my friends, you know, just keep going, keep moving, keep doing it," "I had full support," and "my sister hyper-supports this." Others mentioned an absence of support from friends or family making statements including "well, whatever you want to do mom" or "are you sure you want to do this?". This did not seem to deter their participation 
in the group-based class, potentially due to the wealth of social support experienced from the class and its constituents. Comments on social support relating to the exercise group were interjected throughout the focus group commentary including Pamela's statement: "I wanted to come see the people...you all were a big draw for me."

Fear of failure and illness were also noted as reasons for considering non-participation. Five of the 14 focus group members alluded to the fear of an inability to maintain or meet the standards of the exercise sessions sharing comments such as "I struggled very much with the different steps" or "sometimes I felt like I was working really hard and the heart rate monitor said I wasn't," while three cited that the only classes that they missed were due to illness. Though none of the participants declared missing a class due to fear of failure, they deemed the topic important enough to discuss during the focus group.

Dialogue concerning barrier removal took place in conjunction with the topic of barriers. Participants rendered many of their barriers null and void due to the perceived benefits associated with the aquatic exercise program and management of their potential perceived barriers (health improvement, removal of negative sensations associated with exercise, physical ability to participate, free access, multiple session availability, and retirement status). This binary discussion may exhibit the level of worth associated with the aquatic intervention program along with positively shifting the motivation equation.

Motivation. Motivation for participation in and completion of the aquatic intervention revealed individual and diverse factors that were grouped into several categories. The primary motive for both initiation and completion of the program was health-derived. Both fear of health decline and perceived improvements in health status were topics of discussion that received great attention, and accordingly, was deemed to be of considerable importance collectively across both 
focus groups. Mention of watching family members, friends, and parents physically decline with age, receiving directives from personal physicians, having aspirations to improve or slow physical and mental decay, and perceiving improvements in health and wellness in accordance with the intervention were all discussed a great deal. As Bob articulated: "I've seen my parents and my mom was too sore to move around and she doesn't move around, and her joints are all frozen up now so I just think it's [exercise] a necessary part of life." This health-focused orientation as a motivator to complete the program was evidenced repeatedly, e.g., "my doctor recommended exercise" and "I know exercise has always been good for my medical conditions."

Other factors assisting with initiation of aquatic exercise participation included pursuit of knowledge, program and facility accessibility, and perceived status level or prestige associated with the program. Pursuit of knowledge ranged from learning about one's self to obtaining a new skill. Knowledge was also a compounding variable to accessibility. Participants learned of the viable opportunity to grant them access to a free program. The absence of a fee for the aquatic exercise program was a driving motivator for many of the participants. Over half of the focus group attendees reported that price was a factor in their ability and willingness to participate, making comments such as "the price was definitely right." After program completion, this motivator may have become a barrier due to lack of free programming accessibility. Status and prestige perceived by the participants was stated to be due to the program's tie with West Virginia University and their facility. One participant is quoted to terming the WVU Natatorium as "sacred ground" in which she felt "blessed" to be able to use as a community member.

Individuals also reported that the novel nature of the exercise program assisted in both commencement and continuance of the aquatic exercise sessions. This was evidenced by 
remarks such as "I didn't even feel like I was exercising” or “it was exercise but you really enjoyed it." Tying the idea of fun to exercise seemed novel to this group of once sedentary individuals, but by fostering variation of training, removal of negatively associated exercise stimuli, social interaction, and inviting instructional tactics, almost all of the participants professed that they had fun while participating in the exercise sessions. "I like exercise that's fun." Socialization, in and of itself, was reported as an enormous motivator for a large contingency of the group members. The participants provided social opportunity, support, motivation, and an occasion to expand their social circle. "All the people you are with have a lot to do with it [fun] too and all of these people are so nice. I enjoy coming and talking with them." It was noted many times throughout the focus group that social climate was one of the primary motivators for class attendance.

As derived from the literature and the focus groups, motives and barriers can often be one and the same. Within the confines of this these focus groups in which 14 individuals participated; there were 3 notable overlaps between motivators and barriers: health, support, and accessibility. These findings continue to support the need for a more complex and comprehensive directive for physical activity programming for the aging adult.

Self-Efficacy. To assess self-efficacy participants were asked to rate their confidence level in relation to participation in aquatic exercise. Ten situations were presented with a corresponding likert scale ranging from 1-5 where $1=$ not at all confident and $5=$ extremely confident (Appendix F). The control group recorded corresponding pre-and post-test mean values of $3.96(\mathrm{SD}=0.84)$ and $3.53(\mathrm{SD}=0.74)$; the intervention group's mean pre-, mid-, and post-test values were $3.82(\mathrm{SD}=0.72), 3.61(\mathrm{SD}=0.50)$, and $3.68(\mathrm{SD}=0.75)$, respectively (Table 4.11). All values are very high denoting that both the control group and the intervention 
group had a high level of confidence concerning their ability to participate in aquatic exercise entering the study and that level did not falter throughout the 10-week period showing no interaction between intervention and control groups over time $[\mathrm{F}(1,32)=0.318, \mathrm{p}=0.577, \mathrm{SE}=$ 0.010]. This finding could have implications for exercise adherence and continuation. Though there was not an increase in self-efficacy, as commonly noted in the literature, the maintenance of a positive mean rating is promising (McAuley, Lox, \& Duncan, 1993; McAuley et al., 2003).

During the focus groups three primary categories pertaining to self-efficacy were identified: (1) health and function, (2) fear of water, and (3) knowledge. Health and ability level were given the most attention by the group members, alluding to its perceived level of importance. Improvements in health and ability level were apparent to $100 \%$ of the focus group attendees ranging in impact on activities of daily living. All of the focus group participants stated enhancements in their ease of completing activities that were once considered burdensome including statements such as "walking feels easier to do," "I don't have to wear my brace to do stairs," and "since I've been coming to this, I've had more movement." Specific examples given included shoveling snow, raking leaves, walking up stairs, general ambulation, coordinated movements, and being able to bend and move with greater flexibility.

Fear of water could be construed as a barrier but in the context of the focus group recordings and transcripts, was identified to have a better fit with its effect on self-efficacy. Several participants expressed a fear of water and then paired their fear-related comment with a statement pertaining to their improved comfort level in the aquatic environment or how the aquatic exercise has helped them. Susan provided a good example with her statement: "I can't swim a lick, but I love it and after I got comfortable and turned the wall loose...I love it and really enjoy it." The accounts were always tied to one another, as if the participant was 
expressing their sense of pride that they were able to overcome their fear in order to reap the benefits of the presented opportunity.

Knowledge had two derivatives expressed by the focus groups: (1) seeking knowledge for self-improvement and (2) the non-necessity of knowledge for exercise participation. The very separate camps of thought presented an interesting dichotomy: individuals seeking more knowledge so that they could continue to do self-directed exercise ("with my new knowledge of what exercise is from the pool [joining the gym] will be more beneficial") and others that felt that they didn't need the knowledge in order to reap the benefits ("I liked that fact that the class was structured....they were there telling us what to do"). Though both presented very different knowledge-related goals, it seems that members of the focus groups needs were met with the inclusion of the aquatic exercise intervention.

Social Support. In evaluating the survey questions related to social support, a moderate power statistically significant between subjects group effect was found $[\mathrm{F}(1,32)=5.39, \mathrm{p}=$ 0.027, $\mathrm{SE}=0.03]$. Results show a reduction in perceived social support by the control group with maintenance of social support in the intervention group as seen in Figure 4.8 below. This interesting finding supports that the intervention group maintained their social support systems throughout the 10-week intervention period while the control group perceived a loss of social support. 
Figure 4.8: Pre-Post Scores for Social Support

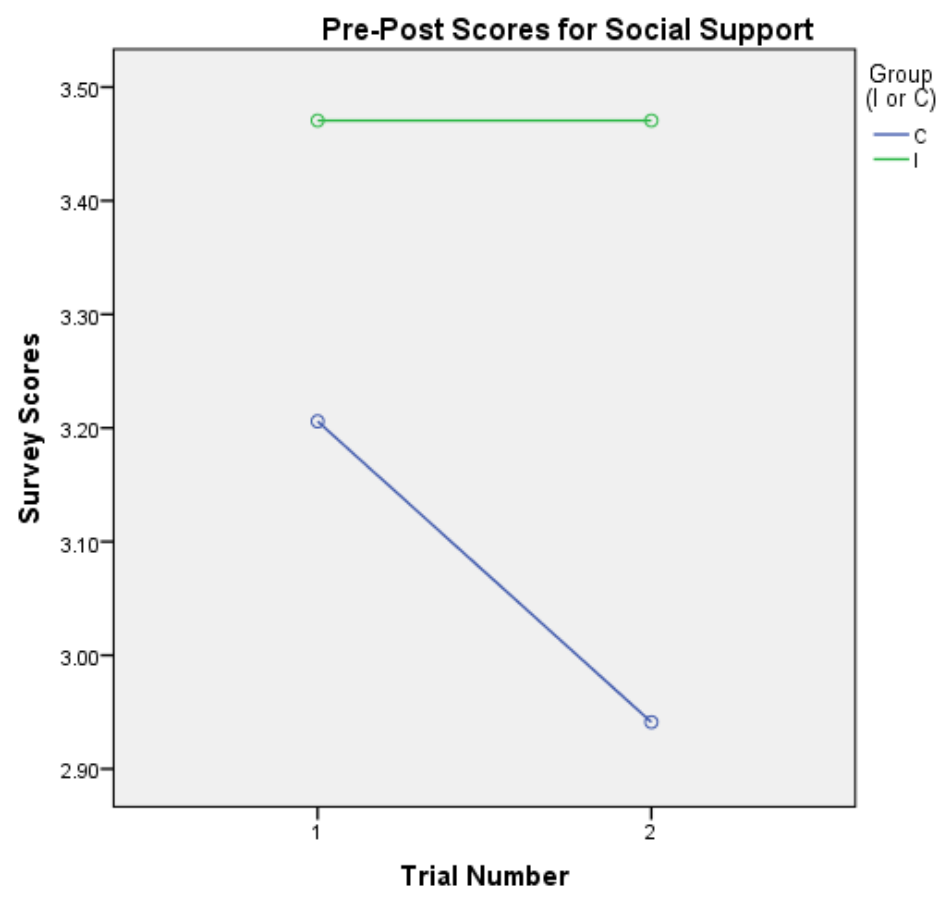

All areas of social support were accounted for in the focus groups. Instrumental, informational, emotional and appraising forms of support were documented through various mediums. Social support provided by the instructors, family, friends, as well as the exercise group each, individually supported the participants with the complete social support spectrum. The participants professed an abundance of support provided by the above mentioned constituents while also revealing how friends, family, or doctors assisted in the informational element that propelled them to join the study. It seems that the vast majority of the focus group participants had an overwhelmingly positive and powerful social support for the entirety of their intervention. Participants made comments about the instructors: "she just encouraged and pushed us to the place where it makes you want to do more," the group: "I haven't seen anything but support...from the other participants and the students [instructors]," and their families: "my entire family does [supports]. My girls, they were just as happy as can be that we were both 
doing it [exercise]." This sound support system may attribute to the small study attrition rate and high attendance levels.

As evident by the quantitative values, no statistically significant difference was noted in any of the psychosocial mediator variables aside from a statistically significant group effect for the control group regarding social support. These findings may be due to the participant's initial ratings beginning at such a high, positive level and thus experiencing a ceiling effect with the majority of survey responses. Both groups maintained a predominantly positive outlook, but with two very different life-experiences. The control group maintained their positive prospective without challenging themselves with a lifestyle change (chronic exercise); while the intervention group maintained their belief levels even when experiencing the lifestyle modification of added exercise. This provides two very different lenses from which the survey was completed.

The focus groups provided a deeper insight to all of the psychosocial mediators addressed in this study. Barriers were accounted for yet traversed with solid coping strategies; motivation was high within multiple factors producing great motive for program commencement and continuation; self-efficacy was positively perpetuated throughout the course of the intervention via health outcomes and ability levels; and social support was strong through multiple cohort channels. All of these positively associated psychosocial mediators only show promise for continued exercise participation.

Triangulation for RQ4. Through triangulation, the affirmation of quantitative and qualitative data convergence was realized. A matrix comparing qualitative and quantitative findings was constructed for comparative purposes (Appendix L). Intervention participants reported low perceived barriers associated with exercise in which the qualitative findings supported this evidence. Participants were able to navigate around most barriers that arose 
during the course of the intervention period as seen by their attendance records (mean of 91\%), mean total barriers $(2.09, \mathrm{SD}=0.38$ where 1 related to no barrier at all), and qualitative statements. The intervention group reported very high levels of motivation towards the presented motives (prevention or reducing pain \& illness, safe and close place to exercise, advice from doctor, and information about health benefits to exercise) with a mean rating of $4.57(\mathrm{SD}=$ 0.81) where 5 is the highest possible score. Interestingly, each of these topics was discussed during the focus group with a high frequency and diversity regardless of question focus.

Participants self-reported that they had lots of social support on the psychosocial mediator survey where a mean answer of $3.65(\mathrm{SD}=0.70)$ was given by the intervention group. As discussed previously, comments concerning the level of social support provided to the intervention group participants spanned the gamut of social support variables. Traversing through instrumental, informational, emotional, and appraising forms of social support, over 70 separate comments were made by a large constituency of the focus group participants in relation to the instructor, fellow exercise participants, family, friends, and doctors. The final psychosocial mediator, selfefficacy, was viewed in two separate lights: perceived self-efficacy level as well as barriers to self-efficacy. Participants rated themselves as having high self-efficacy towards participating in aquatic exercise with a mean value of $3.29(\mathrm{SD}=0.67)$ where 4 is the highest possible rating and perceived there barriers to self-efficacy as being very low $(3.68, \mathrm{SD}=0.75$ where 4 relates to no barriers to self-efficacy). Focus group transcripts confirm this. Though some barriers to selfefficacy were discussed: lack of weight loss, struggles with coordination, effort and perception in disagreement, fear of water (11 comments in total), they were often paired with a confounding statement on how that barrier was removed or diminished throughout the course of the intervention. It is also notable that comments related to self-efficacy were overwhelmingly 
positive with 38 separate statements being made with contributions being made by $100 \%$ of the focus group attendees. This wide scope of commentary propels the researcher to believe that the quantitative and qualitative data converge to show a high level of trustworthiness for the qualitative findings and greater validity for the quantitative results in conjunction with the intervention group. As evident through the qualitative and quantitative findings, psychosocial mediators for the intervention group were all very positive in relation to participant perception. Few negative comments were posed during the focus group but this could be due to response bias commonly seen in focus group settings.

\section{Research Question Five}

RQ5: Does the infusion of adult education theory into a 10-week aquatic exercise class assist in behavior change?

Focus groups were utilized to answer RQ5. The moderator-guided focus group was comprised of a series of canned questions relating to the researchers inquiries (Appendix H). Participant responses throughout the entirety of the focus group transcripts were reviewed for relevance to RQ5, however, specified questions including: "Now that you have completed the program, how do you feel about exercise?" and "Do you think that you will continue to exercise now that the program is over?" were dedicated to seeking knowledge related to RQ5.

Reviewing previous research in combination with current literature on adult education and its relation to exercise intervention strategies for aging adults led to a multifaceted approach to the aquatic exercise intervention. Though providing a sound aquatic exercise program that would foster improved fitness gains was of paramount importance, inclusion of factors associated with a more permanent behavior change was also a dominating goal.

Exercise and Adult Education Framework. In order to achieve behavior change three encompassing variables were considered: (1) finding motivation to begin exercise, (2) begin 
integrating exercise that fits into individual lifestyles, and (3) maintain and gain on all fitness parameters. Each of these steps was viewed through a lens based on adult learning theory when being planned and implemented.

Finding Motivation to Begin Exercise for Older Adults. Due to findings that support a high relation of exercise barriers and motivators, great care was taken in participant recruitment. Educational workshops were offered at a variety of senior centers, assisted living establishments, and other venues catering to aging adults, emphasizing known exercise-initializing motivators. In addition to this, flyers and advertisements highlighting free health-related screening along with details about the aquatic exercise intervention were posted. Findings from the focus groups solidified the motivation for participants was predominantly based on health-related considerations. Among the individualized list of reasons for joining the program, common motives mirrored those mentioned in the literature: health, increased time availability, being educated or referred by a physician to exercise, basic exercise knowledge, and proximity to exercise facilities (Cohen-Mansfield et al., 2006; Schutzer \& Graves, 2004; Schutzer \& Graves, 2004).

Integrating an Exercise Program for Older Adults. The suggestion that a complex behavior becomes more attainable when broken down into smaller, more achievable segments was taken into account when designing the aquatic exercise intervention (Michael, 2003; Skinner $\mathrm{BF}, 1953)$. The concept of gradual progression of the exercise program to eventually meet the ACSM's recommendation of 150 minutes of moderate intensity exercise per week utilized this concept. The physiological principal of progressive overload mirrors this behavioral theory and thus the marriage of the two concepts seems logical. Participant comments noted their growth in 
self-efficacy with the progression of movement difficulty and exercise intensity; some recognized the continual evolution of the program while others did not. One participant states: "my coordination isn't the best in the world, and I find by exercise or repetition like we did in the class, that I saw a big difference at the end of the class than in the beginning. In the end, I felt I was much better in following."

Many participants made comments pertaining to how aquatic exercise improved their selfefficacy for exercise as a whole while providing health benefits that were manifested through their daily activities.

Program planning took group dynamics into great consideration. Providing a positive learning climate as well as posing educational components to extend the intervention groups ambition to continue exercise was a focus. The group dynamics were fostered by engaging the intervention participants in conversations that allowed for them to get to know one another in a more personal fashion. The instructor directed open-ended questions to the group in a conversational manner that encouraged dialogue amongst participants. In accordance with the care given to provide this positive group dynamic, many remarks pertaining to social support received from the exercise group and instructors were made. All of the focus group attendees $(100 \%)$ made at least one positive comment about their rapport with one another or the trainers and how that affected their attendance: "you just felt so comfortable with them [instructors]," "she [instructor] accepted us as individuals," "I wanted to come see the people [other exercise participants]. You all were a big draw for me." The groups shared stories, personal attributes of their lives, and supported one another throughout the intervention and were open to make comments about these communal moments. Additionally, educational components were introduced in a conversational manner to make the learning transparent. Information pertaining 
to how the exercise related to their activities of daily living or how it would assist in their overall health improvement was highlighted while performing the exercise. This informal approach to information sharing afforded the participants the choice of joining in the learning experience via contributing to the conversation without pressure to answer a question or respond to a statement. For example, when performing single and double leg squats, the instructor cued the movement by saying "sit in the chair, then stand-up." This simple cue allowed the participants to relate the movement to an action that they perform on a daily basis. It also provoked conversation about the participant's personal level of difficulty associated to the action. Moreover, the perception of a safe and accepting environment provided the participants an opportunity to grow their selfefficacy in relation to exercise (Bandura, 2001).

Maintain and Gain on Fitness Parameters for Older Adults. Participant completion of the aquatic exercise intervention was an immediate and initial goal of the researcher (evident for research outcomes and study success). A secondary goal was to provide an opportunity for individuals to achieve success with physical activity while educating them on the potential gains that may be achieved to promote continuance of exercise. Program planning took into account the necessity of a lasting behavioral change resulting in perpetuation of exercise involvement. Physiological gains attributed to physical activity have been noted to begin to deteriorate in fewer than 10 days if physical activity does not persist; this underscores the level of importance for exercise maintenance (Madsen, Pedersen, Djurhuus, \& Klitgaard, 1993).

During the entirety of the study, the intervention group showed little attrition credited to self-choice ( 2 participants, $10 \%$ of all) while four individuals dropped out of the study due to medical reasons upon the advice of their personal physician ( 2 hip replacements, 1 congestive heart failure, 1 insertion of cardiac stents). The small self-selected attrition rate provided 
promise for a lasting behavior change. This speculation was confirmed by findings derived from the focus groups. Of the 14 focus group participants, 12 (86\%) commented on strategic plans for continuation of exercise after the study was complete. Plans ranging from sustaining aquatic exercise by joining a paid program to incorporating walking with a spouse or joining an established, free, senior-oriented program. It is important to articulate that these individuals had very specific strategies for exercise continuation suggesting a greater likelihood for actual perpetuation of habitual physical activity. Specifications such as "we are going to join the aerobics class, the paid part" to "there is a class 2 days per week I will probably continue that either next month or the first of the year for sure" were made by a large contingency of the group. During these telling remarks, many of the individuals were verbally providing a needs assessment. They would discuss how the aquatic exercise benefited them most and followed those statements with need for continued improvement, concluding with their plan of action on how to achieve their personal goals within the confines of their lifestyle. For example Tina begins by stating "we are going to join the aerobics class, the paid part" then follows with "There is a difference" in reference to her health and daily life, then finishes with "Still got a long way to go" showing a pattern of plan for physical activity, reasoning for the plan, and what 'requirements' still need to be met.

Lifestyle changes were also well documented from the focus group transcripts. Lifestyle change had four distinct subheadings: (1) lifestyle pattern shift, (2) ability level transformation, (3) prioritization transferal, and (4) retirement. Multiple explanations were given by the participants illuminating a shift in their lifestyle patterns due to physical activity. Some of these shifts were attributed to time allowance for exercise while others were daily activity shifts due to enhanced energy and ability. In 100\% of the focus group attendees, examples of enhanced 
ability levels resulting from the intervention were exposed. Noted changes included health promotion and emotional endorsement with specific citations of improved ability to perform a multitude of activities of daily living. Comments including "it gives me energy," "it helps you to do your activities and everything at one time," and 'it got me out of bed earlier...so it got me moving quicker." One comment that stood out from the collection of comments related to lifestyle shift was from Patty:

When I lost my husband...continuing to walk, I just couldn't get out and walk and then going to an empty house, that's where it all just stopped. I did other things, but I didn't exercise. So I'm using this [study] as motivation to get myself back.

Prioritization transferal was evident throughout the commentary which the researcher attributed to participant perception of health-related changes in addition to their proclaimed commitment to the study. Rearrangement of schedules, discovering solutions for perceived barriers, and dismissal of opportunities that would hinder their attendance were all noted to take place throughout the group. The final lifestyle change repeatedly mentioned was retirement. Retirement posed an interesting dichotic relation with physical activity. Many members of the focus group noted that their will to participate in exercise was reduced upon retirement, while others noted that it supplied the provision of time necessary to take part in the program. "I just retired...instead of sitting at home on the couch I might as well do something" or "I just retired in June and it was a really good way to have discipline in my life" in comparison with "I used to walk quite a bit with my dog, and she got older and I got older and retired so I just quite doing anything."

It seems that through the information provided by the focus group that a behavior change did occur for the vast majority of the intervention group. With approximately $86 \%$ of the 
attendees devising a plan for continuation of physical activity in conjunction with multiple variations in lifestyle changes and benefit recognition, it seems that the intervention group may be newly initiated chronic exercisers.

\section{Research Question Six}

RQ6: Does aquatic exercise provide participants with greater accessibility to exercise participation?

Qualitative findings from the focus group were used to address RQ6. Participant responses in relation to questions concerning alterations in daily activities as well as motivators and barriers were intended to draw out commentary related to accessibility. All focus group comments were reviewed for relevance to RQ6 and were organized into emergent categories.

As previously discussed, physical barriers to participating in exercise are one of the most noted reasons elderly individuals do not exercise (Booth et al., 1997; Cohen-Mansfield, 2003; Schutzer \& Graves, 2004). It was hypothesized that exercising in the water could provide the aging population with a valuable alternative to seemingly inaccessible land exercise. Upon focus group analysis, one primary category of gateway exercise fell out of the underlying theme of accessibility that almost all other comments could reside under. Utilizing aquatic exercise as a gateway exercise to provide knowledge, improve health and function, and improve self-efficacy while reducing barriers to exercise were all distinguishable reasons for using aquatic training as a precursor to other forms of exercise. Focus group attendees commented on obtaining self-related knowledge, acquisition of a new skill, and the absence for the need of preliminary training attributed to the aquatic intervention. They professed a reduction in fear of falling due to initially present balance deficits, ease of movement and accentuation of range of motion, and a heightened ability for participation with existing joint dysfunction. All of these positive attributes associated with the aquatic intervention promotes the belief that it did, in fact, increase 
the groups accessibility to physical activity. Quotes from the focus group provide a more personal and complete picture of how aquatic exercise was able to offer the option of physical activity to those that perceived that they were not able to partake in exercise.

Pamela: I used to walk quite a bit with my dog, and she got older and I got older and retired, and I've got extensive arthritis so I just quite doing anything, which obviously didn't make me feel very good. I hurt my knee badly 2 years ago and I wasn't able to do anything and I thought maybe in the water I could move it. So I saw this advertised and thought I'll try it. And I don't like pools, and I don't swim, and I don't like water (laughs), but I thought I'd try it. And it made my knee better. I don't have to wear my brace to do stairs, so I'm very pleased that I tried it.

Susan: I know exercise has always been good for my medical conditions, but that was not enough to actually get me out and exercise. I have 2 or 3 different machines at home, but I would never get on them. Since I've been doing this water aerobics and I saw those steps out there and knew there were more steps in here I thought oh my God I'm never going to make it up those steps because of my knees, but I had no trouble with my knees at all coming up all of those steps. I know how important it is.

Tina: And I have a lot of arthritis, so I find it difficult to try on land. And I had never done aerobic exercise, but I'm sold and bought. So, I love it, and I see the difference I can do things I couldn't do.

Pam: I like the fact that I can do the same kind of exercises that I can do on ground in the water and I can use more exertion and have less effect on my joints, I mean I could get the exercise I needed but it didn't hurt or it wasn't as hard to do as it would be if I was doing the exercises on the ground. 
Using the aquatic environment as a gateway exercise to prepare the body for other types of training could be a powerful tool to initiate and promote continuance of physical activity. This could be of particular importance for individuals that have compromised balance associated with a fear of falling, joint dysfunction, generalized pain, or sedentary individuals. As the focus group participants denoted, there was not only a shift in their ability level to complete the exercise sessions, there was a change in their ability to participate in other types of physical activity. These very simplistic comments could promote a change in recommendations for physical activity for aging adults with physical impairments. Given the need for physical activity promotion in order to reduce health cost and improve quality of life, integration of aquatic exercise to initiate physical activity in this population should be explored. 


\section{Chapter Five \\ Summary, Conclusions, Implications \& Recommendations}

\section{Summary}

The aging adult population has become a primary focus in the United States due to the increase in longevity and the rapidly escalating number of older Americans. Currently, older adults comprise approximately 13\% (39.6 million) of the population but that number is expected to grow to $19 \%$ (72.1 million) by the year 2030 (Administration on Aging, 2011). With the expected boom in the elderly, the nation is looking towards impending issues and implications

directly related to the older adult. One of the primary concerns is the impact that this population shift will have on our healthcare system, potentially placing unprecedented demands on agerelated and health care services. For this reason programs promoting healthy lifestyles and behaviors, clinical preventative services, education on planning for serious illness, mental health, and deterrence or impedance of cognitive impairment are being investigated, created, and promoted (CDC, 2011).

Participation in exercise and the inclusion of regular physical activity is noted to elicit positive results affecting many of the expressed concerns regarding advancing age. For this reason, exercise has become an important intervention strategy against the negative physical and cognitive outcomes commonly associated with aging. The literature articulates the ability for land-based exercise to improve many facets of executive function in the aging adult (LiuAmbrose et al., 2010; Baker et al., 2010; Barnes et al., 2003; Bielak, 2010; Budde et al., 2008; Davis et al., 2007; Davis et al., 2007; Davis et al., 2008; Liu-Ambrose et al., 2008; Swardfager et al., 2010; Teixeira et al., 2012; Hogan et al., 2013; Joyce et al., 2014). Barriers to participation in land exercise exist within this population with poor health, including pain and illness, noted as the primary contributor to sedentary behavior in the elderly (Booth et al., 1997; Cohen- 
Mansfield, 2003; Schutzer \& Graves, 2004). Due to the often deleterious cognitive and somatic effects of the aging process, the need for other modes of exercise may become necessary in order to employ more inclusive participation ability. Exercising in the water can provide the aging population with a valuable alternative to seemingly inaccessible land exercise. The physical properties of water can be utilized and manipulated to offer support, assistance, and resistance depending on the needs of the individual. The aquatic environment can provide an atmosphere that reduces risk of injury and pain, delivers physiological support for those with less than optimal health status, and, with the inclusion of a group fitness class with a qualified instructor, can provide social support and exercise education (Becker BE \& AJ Cole, 2011; Belza et al., 2002).

For these reasons, the principal concern of this study was to investigate the relation between aquatic-based exercise and executive function in older adults. Due to the complexity of aging adult needs, a more comprehensive vantage point was deemed necessary. Other areas of concentration included psychosocial mediators, exercise accessibility, adult education theory, physical activity level, and measurement of change in fitness and wellness parameters.

\section{Research Questions}

Six research questions (RQ) were investigated. RQ1 through RQ4 were accompanied by corresponding null hypotheses $\left(\mathrm{H}_{\mathrm{O}}\right)$ and RQ5 and RQ6 were purely qualitative in nature. All six RQs are outlined below.

RQ1: Does participation in a 10-week aquatic exercise class significantly improve $(\mathrm{p} \leq .05)$ cognitive function when compared to the control group? 
RQ2: Does participation in a 10-week aquatic exercise class significantly improve $(\mathrm{p} \leq .05)$ cardiovascular fitness when utilizing the Kruel target heart rate deduction to configure individualized target heart rate zones when compared to the control group?

RQ3: Do significant $(\mathrm{p} \leq .05)$ positive changes occur for general fitness and wellness parameters after participation in a 10-week aquatic exercise class when compared to the control group?

RQ4: Does participation in a 10-week aquatic exercise class significantly alter $(\mathrm{p} \leq .05)$ social support, self-efficacy, motivators, or exercise barriers when compared to the control group?

RQ5: Does the infusion of adult education theory into a 10-week aquatic exercise class assist in behavior change?

RQ6: Does aquatic exercise provide participants with greater accessibility to exercise participation?

\section{Methodology}

Participants recruited for this study were a sample of convenience, required to be between the ages of 60-90 years with no neurological defect (e.g., possible dementia, self-report of neurological disease such as multiple sclerosis, brain tumor, and Parkinson's disease), leading a sedentary lifestyle (<150 minutes exercise per week), and be willing and able to participate in an aquatic exercise program. A total of forty-four participants qualified and began the study $(\mathrm{N}=$ 44) while a total of $34(n=34)$ completed the research study in full $(n=17$ in both intervention and control groups).

All participants attended three preliminary visits that were comprised of various assessments separated to provide either familiarization for the increasing testing validity, 
reduction of respondent fatigue, or appropriate testing provisions. All participants were required to obtain medical clearance from their physician to participate in the exercise intervention and all of the accompanying assessments to promote safety and wellbeing for the participants.

During visit 1 , participants were taken through a familiarization session with CANTAB®, completed a health history, and provided their demographic data. One week following visit 1, participants returned to allow collection of anthropometric measurements and complete the CANTAB battery to collect baseline measurements for cognitive function. During visit 3, one week prior to the exercise intervention, the final baseline assessments of the 6-minute walk test, RHR, RBP, and psychosocial mediator surveys were completed. After the final visit, participants were arranged into matched pairs by utilizing age and fitness parameters. One participant from each pair was then randomly assigned to either the treatment or control group. Members of the treatment group began the exercises sessions the week following completion of their preliminary assessments while the control group was asked to maintain their current physical activity level.

The intervention group participated in a 10-week aquatic exercise class 3 times per week. Participants exercise sessions were graded from $45 \%$ heart rate reserve (HRR) increasing to $75 \%$ HRR over the course of the trial utilizing both continuous and interval-based training designs (Appendix D). Intensity was determined by the preliminary exercise testing and observed via the use of heart rate monitors set to participant's individual parameters in conjunction with the Kruel formula for aquatic target heart rate. Adult learning theory was infused into the instruction methods during the classes to promote greater understanding of exercise movements and reason behind the inclusion of the movement. Heart rate and rate of perceived exertion were monitored throughout each exercise session via heart rate monitors and data collection sheets. At week-5 of 
the exercise intervention (mid-intervention), participants were given the psychosocial mediator surveys once more. At the end of their final exercise session, the intervention group members were asked to take part in one of two focus group sessions offered in accordance with the two exercise class times.

At the conclusion of the 10-week exercise intervention, both treatment and control group participants were asked to join in a final visit. The visit took place during the week immediately following the conclusion of the exercise intervention. The post-intervention visit consisted of collecting participant's anthropometric measurements, resting heart rate, resting blood pressure, noting any changes in health history, and completion of 3 psychosocial mediator surveys. Cognitive screening using the $\mathrm{CANTAB} \circledast$ was conducted to reassess working memory capacity, spatial working memory, orientation, registration, attention, calculation, language and recall. Lastly, participants were asked to complete a 6-minute walk test.

Preliminary and post-intervention quantitative measurements assessing physiological parameters (anthropometric measurements, RHR, RBP, and distance walked in 6 minutes) along with psychosocial mediator survey results (exercise barriers, social support, self-efficacy, and motivators) were analyzed using SPSS software (version 21.0, SPSS Inc., Chicago, Illinois) with a two-way ANOVA. Statistical data was assessed to test the null hypotheses $\left(\mathrm{H}_{01}-\mathrm{H}_{04}\right)$ as an answer to corresponding research questions RQ1 - RQ4.

Content analysis with the inclusion of frequency and inductive analysis of the focus groups were employed to answer RQ5 and RQ6. Data derived from these groups were also used to triangulate the qualitative and quantitative findings associated with RQ1 - RQ4. 


\section{Findings}

A two-way ANOVA revealed that the aquatic exercise intervention did not have global effects on the cognitive function parameters tested by the CANTAB battery. Of the five cognitive assessments, only spatial working memory (SWM) revealed a statistically significant $(p=0.014)$ change from baseline measurements when comparing the control and intervention groups. Paired associated learning (PAL) as well as AST showed statistically significant time effects for both the intervention and control groups suggesting a learning effect while all other tests included in the battery (OTS and RTI) showed no effects. Method triangulation was performed with quantitative and qualitative data to derive more validity and truth value to the findings. Upon comparison, qualitative and quantitative data converged to denote no pertinent global change to executive function while displaying improvements in cognitive parameters aligned with SWM (Appendix J).

In addition to the cognitive variables, physiological variables also elicited mixed results. Though the 6-minute walk distance did not show a statistically significant change, the control group displayed a reduction in distance while the intervention group improved slightly. A similar pattern emerged in relation to the SBP: slight improvement (decrease in SBP) in the intervention group compared to a small increase in the control group. Statistically significant positive changes were observed in the DBP $(p=0.014)$ for the intervention group. The intervention group's DBP was substantially lower at the post-intervention visit in comparison to their pre-intervention visit while the control group showed an increase in DBP at post-test. Other quantitative wellness parameters assessed, weight $(\mathrm{p}=0.289)$ and body composition $(\mathrm{p}=0.56)$, showed no statistically significant interactions. Qualitatively, however, 100\% of focus group participants made comments pertaining to overall wellbeing and improved quality of life. 
Triangulation of the two data sets was conducted to recognize the level of divergence and convergence. A matrix was developed to illustrate findings from the different datasets related to wellness (Appendix K). Qualitative data was assessed with both frequency and inductive analysis for distance walked and wellness parameters. A lack of commentary concerning changes in body weight and body composition reflected the negligible quantitative change in these parameters also denoting convergence of data.

In relation to psychosocial mediators, no statistically significant interactions were found. Initial survey results revealed very low perceived barriers, high motivation, sound social support, and high self-efficacy from both the intervention and control groups presenting a potential ceiling effect upon post-test findings. Qualitative data corroborated these findings for the intervention group with predominantly positive, voluminous responses in reference to all psychosocial mediators discussed. Barriers were accounted for yet traversed with solid coping strategies; motivation was high within multiple factors producing great motive for program commencement and continuation; self-efficacy was positively perpetuated throughout the course of the intervention via health outcomes and ability levels; and social support was strong through multiple cohort channels. All of these positively associated psychosocial mediators only show promise for continued exercise participation. Further, triangulation of qualitative and quantitative findings pertaining to psychosocial mediators demonstrated data convergence affirming that the intervention group had low perceived barriers to exercise, high levels of motivation, ample social support from various contingencies, and high perceived self-efficacy with few perceived barriers to self-efficacy.

The exercise intervention was built around an adult education framework consisting of (1) finding motivation to begin exercise, (2) begin integrating exercise that fits into individual 
lifestyles, and (3) maintain and gain on all fitness parameters. It seems that through the information provided by the focus group that a behavior change did occur for the vast majority of the intervention group. With $86 \%$ of the attendees devising a plan for continuation of physical activity in conjunction with multiple variations in lifestyle changes and benefit recognition, it seems that the intervention group may be newly initiated chronic exercisers.

The resolve of the intervention participants to continue exercise may be due in part to their improved accessibility to exercise. Utilizing aquatic exercise as a gateway to provide knowledge, advance health and function, and improve self-efficacy while reducing barriers to exercise were all distinguishable reasons for using aquatic training as a precursor to other forms of exercise. Qualitative findings provide a more personal and complete picture of how aquatic exercise was able to offer the option of physical activity to those with the perception that they were not able to take part in exercise.

\section{Conclusions}

Conclusions for this study based on the major findings are presented by research question. Each subsection will address rationale and interpretation connected to each finding.

\section{Research Question One.}

RQ 1: Research Question One asks: Does participation in a 10-week aquatic exercise class result in a statistically significant $(p \leq .05)$ improvement in cognitive function when compared to the control group?

The majority of executive function parameters tested during this intervention yielded no interaction with the intervention. No statistical significance was found for spatial planning and working memory in relation to OTS, visuo-spatial association via PAL, attention shifting and allocation thru AST, or decisional reaction time by means of RTI. A time effect was observed with PAL as well as AST leading the researcher to believe that a learning effect took place. 
Triangulation with focus group transcripts converged with the quantitative findings that there was no global change in executive function parameters. Another notable aside is that during the RTI post-test, many of the participants experienced technical errors while the assessment was being conducted. Due to this issue, results obtained from the RTI may be null and void.

Though most of the cognitive function tests showed no interaction between the control and intervention groups, the SWM assessment showed positive trends in the intervention group, with one measure reaching statistical significance. SWM measures the ability to actively manipulate spatial information in the working memory. This may denote that exercise distinctively improves higher-level cognitive function necessary for more complicated tasks. These results may also reflect how increased movement diversity may result in better SWM due to linking kinesthetic awareness with spatial-working cerebral activity. Spatial working memory actively maintains and manipulates stored information to plan and guide behavior (Badcock et al., 2005). Thus, if participants diversify their movement abilities, they may be able to plan and guide behavior with less effort and greater success. Emergent qualitative categories converged with this quantitative finding denoting that participants noted improvements in movement quality including walking, balance, and other ADLs.

The investigator concluded that the 10-week aquatic intervention, in comparison to the maintenance of a sedentary lifestyle, showed effects on only a single area measured of executive function. The only cognitive parameter showing statically significant $(\mathrm{p} \leq 0.05)$ improvement was that of spatial working memory. Qualitative findings reinforced the quantitative results denoting no comprehensive cognitive change but a more specified neuromuscular enhancement. The failure to completely reject $\mathrm{Ho}_{1}$ was unanticipated given the depth and breadth of the literature supporting land-based exercises effects on all of the studied executive function 
parameters. Though the intervention structure met the ACSM's guidelines for cardiorespiratory health and wellness, it did not follow the frequency of 5 times per week and duration of 30 minutes that Denkinger (2012) indicated as the gold standard for cognitive interventions. The same total duration was accrued over the period of 3 days versus 5. Other studies reporting positive executive function results with exercise lasting 40-90 minutes were also noted during the construction of the exercise intervention (Davis et al., 2008; Radak et al., 2001; van Uffelen et al., 2009). Due to these diverse findings and the greater likelihood of participation commitment, the researcher chose to assure that the ACSM guidelines were met with the belief that cognitive improvements most substantially hinged on total exercise duration paired with the appropriate intensity (Davis et al., 2008; Angevaren et al., 2010; Fabre et al., 2002).

The modification in exercise medium and altered heart rate response may also have had an effect on the cognitive outcomes. The concept of aquatic exercise and the adaptations needed for operative movement production is novel to those unfamiliar with the effects of water immersion. Initial learning of body mechanics, kinesthetic awareness, and, for some, suppression of aquaphobia were obstacles that had to be overcome during the intervention. These variables may have hindered the individual's ability to participate to their fullest capacity resulting in a muted exercise stimulus.

The physical alterations necessary due to the change in medium and generalized effects of immersion may have also contributed to the lack of statistical significance. Due to an absence of research concerning cognitive function and aquatic exercise, the researcher drew on her knowledge of the environment and known effects of immersion to construct a theory on how exercising in the water would alter executive function. Spatial planning, working memory, visuo-spatial association, attention shifting and allocation, and decisional reaction time were 
areas that showed no improvement post intervention. The researcher could suggest that with the perceived safety of movement in the aquatic environment, these processes were of less concern to the participants thus were not as methodically traversed while exercising. Though complex movements were introduced, the participants were not at risk of falling due to lack of spatial planning, attention shifting, or decisional reaction time. Because the majority of the instructed exercises were done in place, visuo-spatial association may not have been stimulated sufficiently. It is clear that greater research is needed for further understanding of the aquatic environments effects on cognitive functions, thus this area of interest will be discussed in greater detail under future research.

\section{Research Question Two.}

$R Q$ 2: Does participation in a 10-week aquatic exercise class result in a statistically significant $(p \leq .05)$ improvement in cardiovascular fitness when utilizing the Kruel target heart rate deduction to configure individualized target heart rate zones when compared to the control group?

There was not a simplistic response of either accepting or rejecting the null hypothesis $\left(\mathrm{H}_{02}\right)$ that states: Participation in a 10-week aquatic exercise class does not result in a statistically significant improvement $(\mathrm{p} \leq .05)$ in cardiovascular fitness when utilizing the Kruel target heart rate deduction to configure individualized target heart rate zones when compared to the control group. The data collected from the 6-minute walk along with RHR, as well as resting SBP and DBP were used as the primary factors associated to cardiovascular fitness. Though no change was observed with RHR, all other fitness variables showed positive trends for change in the intervention group and the change in DBP reached statistical significance. Due to the small sample size, statistical significance and power may have muted the intervention results thus the quantitative results were used along with qualitative findings for triangulation to allow for the research question to be addressed using all of the data compiled. Quantitative positive changes 
were supplemented with qualitative accounts of improvements in global wellness with particular emphasis on walking ability. Frequency analysis of the focus group transcripts revealed 35 separate comments of pertinence to cardiovascular fitness that reflected $100 \%$ of the focus group participants. Remarks pertaining to improved wellness emerged with 6 walking-specific comments and 24 walking related comments noted. Triangulation of the findings revealed a convergence of the data showing a positive trend in fitness, and more specifically, ambulatory ability.

There are a wide variety of reasons that RHR showed no change during the intervention. The primary factor relating to this is medication usage by the participants. During the health history, medical history was recorded along with current medication dosage. All 17 of the intervention participants were on medications known to regulate HR downward (15 of the 17 control participants were medicated as well). This was taken into consideration with the individualization of the exercise prescription by using the accepted method of subtracting 20 beats per minute $(\mathrm{bpm})$ from the target heart rate. Even with the modification, only one participant from the intervention group was able to maintain their prescribed target heart rate (THR) for $100 \%$ of the intervention period. This brings question to how beta-blockers vary the reaction in the aquatic environment. There are no existing studies that report on the effects of beta-blockers in conjunction with the effects of immersion outside of remarking that individuals on beta blockers should utilize their RPE in place of their THR when configuring exercise intensity. This is an area that needs further research and will be discussed in more depth within the future research segment.

Outside of RHR, the other factors directly relating to cardiovascular fitness -- the 6minute walk test as well as resting SBP and DBP -- all showed favorable trends with DBP 
reaching statistical significance. The investigator concluded that, with the exception of DBP, the null hypothesis $\left(\mathrm{H}_{02}\right)$ that the intervention does not improve cardiovascular fitness at a level of statistical significance $(\mathrm{p} \leq 0.05)$ is accepted. Though statistical significance was not reached, triangulation of cardiovascular fitness variables with qualitative findings indicates a generalized improvement in ambulation ability promoting the belief that improvements were experienced by the participants.

The lack of statistical significance for all cardiovascular fitness parameters was an unexpected manifestation. Literature supports the ability of aquatic exercise to promote cardiovascular fitness in addition to walking speed, thus a statistical improvement in the 6minute walk test was expected (Rica et al., 2013; Sanders, Takeshima, Rogers, Colado, \& Borreani, 2013; Meredith-Jones et al., 2011). Anticipated changes in RHR and resting SBP were not projected to be as substantial due to the medication status of the participants and variation in assessment times. Though considerable alterations have been noted in blood pressure response in relation to aquatic exercise, this is most markedly seen in populations that are not taking medications that affect BP (Rodriguez et al., 2011; Terblanche \& Millen, 2012).

Resting HR, SBP and DBP are independently related to cardiovascular risk stratification; thus changes in these variables show a progression towards improved cardiovascular health (Basile, 2002). Interestingly, elevated RHR is a factor for cardiovascular risk and mortality independent of participation in physical activity and fitness levels (Jensen, Suadicani, Hein, \& Gyntelberg, 2013). Studies have shown that a single beat per minute increase in RHR can increase cardiovascular risk and mortality rate by 1.6-2.2\% (Jensen et al., 2013). With this vantage point, the mean decrease of $1.42 \mathrm{bpm}$ measured in the intervention group may have some clinical impact in relation to cardiovascular health and mortality rate. Likewise, SBP < 
140mmHg has been aligned with lowered cardiovascular risk and mortality (Basile, 2002).

Three of the 17 intervention participants experienced a downward SBP shift to relegate them below their initial $\mathrm{SBP} \geq 140 \mathrm{mmHg}$ while 4 additional individuals had a downward shift in SBP. Though their beginning SBPs was already $\leq 140$, they still experienced a reduction in SBP. Again, clinical implications are seen when viewed through this lens.

\section{Research Question Three.}

RQ3: Do statistically significant ( $p \leq .05)$ positive changes occur for general fitness and wellness parameters after participation in a 10-week aquatic exercise class when compared to the control group?

The null hypothesis for Research Question Three $\left(\mathrm{H}_{03}\right)$ stated that no statistically significant positive changes $(\mathrm{p} \leq 0.05)$ would occur for general fitness and wellness parameters after participation in a 10-week aquatic exercise class when compared to the control group. Statistical findings from the wellness parameters (RHR, SBP, DBP, distance walked, weight, and body composition) revealed no statically significant changes in RHR, SBP, distance walked, weight or body composition. Positive trends in SBP and distance walked were present and DBP reached statistical significance $(\mathrm{p} \leq 0.05)$. Methods triangulation was employed by examining the focus group data for statements related to improved wellness and contrasting these with quantitative findings. Thirty-five separate statements relating to improved wellness, 34 positive and 1 negative, emerged from the focus group transcripts. The comments reinforce that the positive quantitative trends observed with SBP, DBP, and distance walked could be clinically noteworthy (Appendix $\mathrm{K}$ ). Therefore, $\mathrm{H}_{03}$ could not be fully accepted nor rejected.

The parameters of weight loss and body composition (body fat percent) did not show change during the intervention. Though this was not intended to be a weight loss program, the addition of physical activity to the participants' self-reported sedentary lifestyles would have 
been expected to result in a negative energy balance (Calories consumed is less than the Calories expended). Explanations for this outcome may be Caloric compensation by participants post exercise. Caloric compensation, or the replacement of burned Calories with food consumption, has been noted to occur in some exercisers (Werle, Wansink, \& Payne, 2011; King et al., 2007). Often individuals that exercise perceive the need to eat more and often experience a heightened pleasure response to food following a workout (King et al., 2007). Exercise in cold water $\left(20^{\circ} \mathrm{C}\right.$ $22^{\circ} \mathrm{C}$ or $68^{\circ} \mathrm{F}-71^{\circ} \mathrm{F}$ ) has been shown to increase post Caloric consumption by up to $44 \%$ when compared to thermo-neutral water conditions $\left(33^{\circ} \mathrm{C}\right.$ or $\left.91.4^{\circ} \mathrm{F}\right)$ (White, Dressendorfer, Holland, McCoy, \& Ferguson, 2005; Sheldahl, Buskirk, Loomis, Hodgson, \& Mendez, 1982), though this intervention took place in $79-80^{\circ} \mathrm{F}$ water thus should not have had such an immense thermic response to food ingestion but there have been no studies conducted in this temperature to affirm this allegation. The lack of weight loss and body composition change findings from this study are in accordance to others studies with the similar populations and interventions (Rica et al., 2013).

\section{Research Question Four.}

RQ4: Does participation in a 10-week aquatic exercise class result in a statistically significant ( $p \leq .05)$ alteration of social support, self-efficacy, motivators, or exercise barriers when compared to the control group?

The researcher concluded that the null hypothesis for Research Question Four $\left(\mathrm{H}_{04}\right)$ stating that participation in a 10 -week aquatic exercise class does not significantly alter $(\mathrm{p} \geq .05)$ social support, self-efficacy, motivators, or exercise barriers when compared to the control group was accepted. Findings from the preliminary and post-intervention surveys did not reflect statistically significant interactions for the intervention group when compared to the control group. The participants' initial ratings of all psychosocial mediators displayed a very high, 
positive level, and thus a ceiling effect with the survey responses may have been experienced. Very little change was seen over time with the survey results displaying maintenance of selfefficacy, motivators, lack of exercise barriers, and an abundant presence of social support. Social support did show a statistically significant control group effect relating to a noted reduction in social support over the course of the 10-week intervention.

Reasoning behind this result may be attributed to the volunteer's state of readiness upon entering the program. Individuals self-selected to contact the researcher to inquire about program participation denotes a high level of readiness to begin exercise (Prochaska \& Velicer, 1997). In Buckworth and Dishman's (2002) stages of change model depicting a cycle of precontemplation, contemplation, preparation, action, maintenance, and relapse, participants that were interested in the study were probably already in the contemplation, preparation, or action phases (Buckworth \& Dishman, 2002). The psychosocial mediator surveys may have directly reflected that the study participants were at a heightened state of readiness. The interesting component to this is that both groups maintained this positive outlook with two very different life-experiences. The control group maintained their positive prospective without challenging themselves with a lifestyle change (chronic exercise); while the intervention group maintained their belief levels even when experiencing the lifestyle modification of added exercise. This provides two very different lenses from which the survey was completed.

The focus groups provided a deeper insight to all of the psychosocial mediators addressed in this study. Barriers were accounted for yet traversed with solid coping strategies; motivation was high within multiple factors producing great motive for program commencement and continuation; self-efficacy was positively perpetuated throughout the course of the intervention via health outcomes and ability levels; and social support was strong through multiple cohort 
channels. Method triangulation of the quantitative and qualitative findings converged, maintaining that all psychosocial mediators were found to be associated with positive connotations with the intervention group with very few negatively associated comments being made.

Though these statistical findings result in acceptance of the null hypothesis $\left(\mathrm{H}_{04}\right)$, convergence of the intervention groups' data confirmed the survey findings while offering insight into the psychosocial mediators and their development over the course of the 10 -week intervention. The growth and adaptation of the group's ability to support one another along with improvement in self-efficacy was highly noted. An additional finding included the groups' remarks concerning the social support received by the instructor and the improved belief in ability level from family and friends. This could provide clinical value to the level of impact that building a safe, enjoyable learning environment can have on exercise intervention success.

\section{Research Question Five.}

RQ5: Does the infusion of adult education theory into a 10-week aquatic exercise class assist in behavior change?

The investigator concluded that infusion of adult education theory into a 10-week aquatic exercise class did assist in behavior change. Emergent categories from content analysis of the focus groups when paired with the researcher's behavior change framework (finding motivation to begin exercise; begin integrating exercise that fits into individual lifestyles; and maintain and gain on all fitness parameters) reflected a cohesive picture of confident continuation of chronic exercise. Upon frequency analysis, 12 (86\%) of the 14 focus group participants commented on strategic plans for continuation of exercise after the study was complete. It is important to articulate that these individuals had very specific strategies for exercise continuation supplying a greater likelihood for actual perpetuation of habitual physical activity. 
Reasoning to support a more enduring behavior change was evident through benefit recognition and modifications in lifestyle to allow for adaptation and inclusion of physical activity into the participants daily lives. The categories and themes that emerged from the focus group comments pertaining to behavior change prevailed throughout the entirety of the focus group regardless of the question posed to the participants. Comments pertaining to the participants' ultimate continuation of exercise were offered during introductory statements, as answers to many non-behavior focused questions, as well as in the closing remarks. Participants made direct and indirect comments with high relation to behavioral change and their intention for continuation of exercise as well as reasoning for that continuation. The presence of ample behavioral change commentary leads the researcher to assert that the inclusion of adult educational theory initiates sedentary individuals in a manner that assists with a more permanent increase in physical activity participation. This assertion was further supported by literature professing the need for including adult education for program success (Schutzer \& Graves, 2004;

O'Neill \& Reid, 1991; Bauman et al., 2012; Bauman et al., 2002; Sallis JF et al., 2008; SJH Biddle et al., 2012).

\section{Research Question Six.}

RQ6: Does aquatic exercise provide participants with greater accessibility to exercise participation?

Aquatic exercise was concluded to provide participants with greater accessibility to exercise participation in accordance with qualitative findings. As with behavioral change, comments pertaining to the aquatic environments assisting in acquisition of exercise were pervasive in the qualitative findings. The ever-present remarks made by the participants about their lack of ability to participate in land exercise led the researcher to derive the primary theme of utilizing the aquatic environment as a gateway to chronic exercise. This concept was 
consequent to participant comments universally relating back to lack of land-based exercise ability. These findings are supported by a small body of research reporting that aquatic exercise promotes activity level and quality of life (Honda \& Kamioka, 2012; Kamioka et al., 2010; Kamioka et al., 2011). One confounding qualitative finding was that of aquaphobia. Some participants professed a fear of the water, which would implicate a reduction in electing to take part in aquatic exercise, yet the participants refuted that their individualistic alternate motives (pain, medical history, fear of health decline, etc.) were more powerful stimuli than their aquaphobia, almost negating their fear-related comments.

\section{Implications for Practice and Policy}

This study examined the effects of a 10-week aquatic exercise program on cognitive function with a secondary emphasis on fitness and wellness gains, psychosocial mediators in relation to behavior change, and exercise accessibility. The research questions were concerned with global cognitive and physiological changes and how these results related to one another. Findings from this study may assist in refining physical activity programming considerations for older adults. Additionally, a suggestion for the adjustment of societal norms to reduce perceived or societally imposed barriers is considered.

Physical and cognitive benefits derived from engaging in physical activity are well documented, yet there are a lack of statistics that report the incidence of participation in regimented physical activity meeting ACSM guidelines in the aging population. It is estimated that $80 \%$ of adult Americans do not meet the recommended exercise guidelines and though this does not delineate between individuals over the age of 18 and those over the age of 60 , the findings also note that younger adults were the highest reported contingency to meet activity recommendations (cdc.gov, 2013). Other estimates show the prevalence of physical activity 
decline with age, remarking that adherence for individuals over the age of 60 is less than $2.5 \%$ (Haskell et al., 2007; Lloyd-Jones et al., 2010). Additionally, little research has been conducted to determine adherence to an exercise program, once initiated, for older adults in general or those with mobility limitations or chronic disease. Studies that have reported on adherence to physical activity interventions have reported an adherence rate of $60-85 \%$ for long-term trials lasting over 6 months (Ettinger, Jr. et al., 1997; Jette et al., 1999; King, Haskell, Young, Oka, \& Stefanick, 1995; McCartney, Hicks, Martin, \& Webber, 1995). The importance of participation and continuation of physical activity is multifaceted in reference to successful aging. Exercise assists with both physical and cognitive fortifications, which are equally paramount to maintenance of independence. Thus, the level of importance from both a societal (healthcare dollars) and individual (quality of life) view is well established.

Given compelling evidence that individuals over the age of 60 are becoming more and more sedentary with greater losses in cognitive and physical parameters, there is a need to reconsider all components of exercise rationale in order to propel changes to increase physical activity levels in this elderly population. This study's findings offer programming implications concerning accessibility, initiation, and continuation of physical activity for older adults. Implications for practice will be presented from strongest to weakest in relation to research findings and documentation in the literature.

Accessibility of Exercise. Implications towards improving accessibility to physical activity were derived from the findings of this study, including the concept of utilizing aquatic exercise as a gateway to exercise participation. This encompassing theme that emerged from the qualitative findings suggests that aquatic exercise promoted movement diversity and, in turn, enhanced ability-related self-efficacy. The perceived and physiological ability level of 
individuals to participate in exercise seems to diminish with age. Perception of ability level, otherwise denoted as self-efficacy, has been found to be as powerful as actual ability level with an emphasis on environmental properties along with biomechanical restraints and abilities (Konczak, Meeuwsen, \& Cress, 1992). Ability-related self-efficacy has also been noted to change as age progresses as illustrated by Sonstroem and Morgan's exercise and self-belief model (Konczak et al., 1992; Sonstroem \& Morgan, 1989). Physical competence and abilityrelated self-efficacy can be linked to one another with the notion that as one improves or becomes impaired so does the other (McAuley, Blissmer, Katula, Duncan, \& Mihalko, 2000). Thus, if older adults have lower self-efficacy in relation to physical ability levels (balance, ambulation, etc.), they may believe that they cannot safely perform physical activity. Individuals with lower qualitatively-stated self-efficacy (in relation to physical ability resulting from physical limitations or past experiences) were permitted to effectively participate in exercise without pain or concern of further debilitation. Participants were provided the opportunity to learn the biomechanics without the fear of falling, the association of pain, and with buoyant support from the aquatic environment. This allowed the participants to be more experimental with their movement, increase their biomechanical diversity and improve their ability-related self-efficacy. The incorporation of aquatic exercise intervened in the sedentary pathway towards deconditioning and reduced quality of life, causing rerouting and recalibrating of the participants movement ability (Figure 5.1). 
Figure 5.1: Sedentary Lifestyle in Comparison to Introduction of Aquatic Exercise
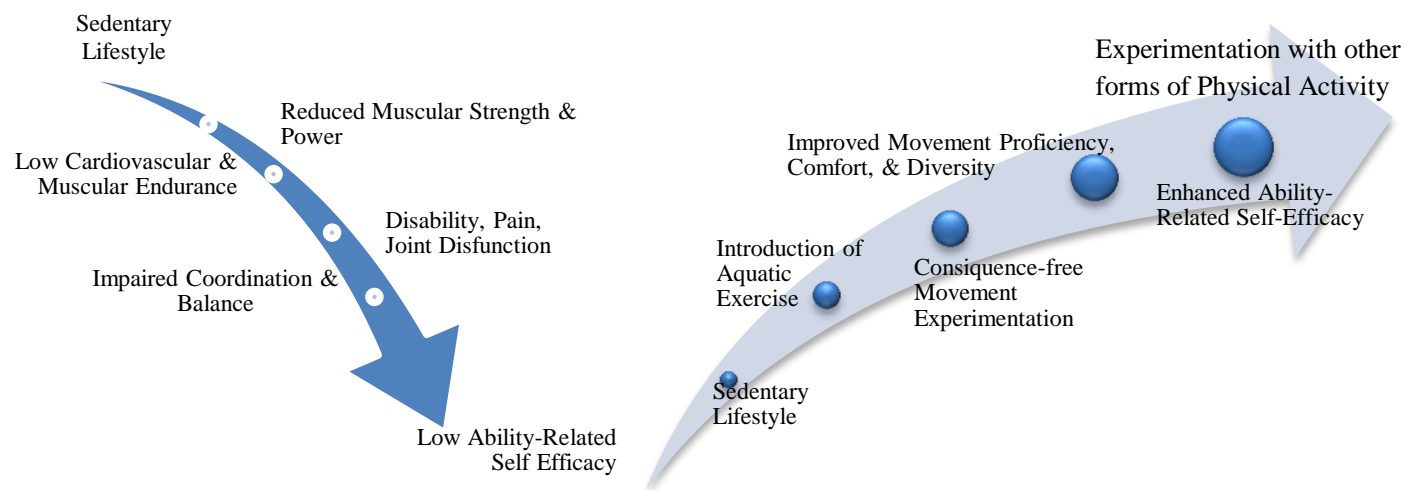

The use of aquatic exercise as a gateway training tool to improve ability related selfefficacy and enhance physical parameters is not a novel concept. Prehabilitation, or pre hab, employs a similar concept: strengthen prior to injury or surgery. Pre hab has been noted to reduce injury, surgical complications, and rehabilitation duration (Jack, West, \& Grocott, 2011; Alkarmi et al., 2010; Swank et al., 2011). The idea of performing preparatory physical training to prepare the body for other types of exercise participation is just an extension of this model.

Movement safety aligns with the gateway concept as well. Many participants noted impairments in balance, posture, and kinesthetic awareness prior to the intervention and the positive effects that the aquatic exercise had on these variables. The perpetuation of enhanced movement diversity through exercise variability and repetition along with neuromuscular adaptations is believed to be the derivation of the said benefits. The allowance of consequencefree movement without the fear of a misstep, fall, or coordination error seems to allow the advancement of physiological parameters resulting in comprehensive movement safety regardless of environment.

In addition to the physical assessments, focus group and cognitive function testing converged to lead the researcher to believe that improvement in behavior planning, goal 
selection, and movement execution improved with aquatic exercise. Inferences that aquatic exercise can promote movement safety via cognitive organization of a movement prior to its execution poses further potential for improvement in movement safety and quality. The properties of the aquatic environment-- buoyancy and viscosity in particular-- naturally slow the movements down from what is normally experienced on land. This could allow for a more complete thought process of the performance of the movement endorsing greater advancements in neuromuscular adaptation. Studies have supported that breaking down the gross movement, enhancing task attention, and repetition of movement patterns advance motor learning and augment ability levels (Chiviacowsky, Wulf, \& Wally, 2010; Chiviacowsky, Campos, \& Domingues, 2010; Chiviacowsky \& Wulf, 2007; Seidler, 2007).

Learning and Skill Acquisition. The ability to learn is the fulcrum of using aquatic exercise as a gateway form of physical activity. The process of learning a new skill has been outlined to require three sequential stages: cognitive, associative, and autonomous (Willingham, Peterson, Manning, \& Brashear, 1997; Fitts, 1992; Smith, Ghazizadeh, \& Shadmehr, 2006; Willingham, 1998). The initial learning stage is noted to be the more cognitively demanding stage of skill acquisition in which the learner attempts to understand the requirements of the skill. During this stage, the learner produces inconsistent skill attempts due to the need for attempting various strategies for success or may even need to verbalize the movement strategies (Adams, 1971; Anguera, Reuter-Lorenz, Willingham, \& Seidler, 2011). Movement errors in route to the ideal pattern have been shown to positively influence the individuals' ability to perform a novel task (Poole, 1991). Evidence of this initial learning process occurring during the aquatic exercise intervention period manifested through qualitative and quantitative data as well as observation. Qualitatively, participants indicated improved movement ability while remarking on how the 
exercises performed during the sessions had become more attainable. Comments pertaining to their improved ability to follow and replicate the intended aquatic exercise movement were also noted. Quantitative variables noted physical and a cognitive improvement in SWM (distinguished to contribute to early learning adaptation) to supplement and corroborate these findings (Anguera, Reuter-Lorenz, Willingham, \& Seidler, 2010; Anguera et al., 2011).

Observations by the researcher denote participants echoing verbal instructions given by the instructor or continuously repeating the pattern verbally. Observations of participants trying new movement patterns also gave light to different movement strategies being attempted before attaining what was considered to be the optimal movement pattern. Thus, the learner attempted to give attention to the relevant skill-related information (Poole, 1991).

As the aquatic exercise sessions progressed, greater skill was required of the participants. Movement diversity as well as intensity were continuously developed throughout the 10-week intervention period. Participants' initial cognitive learning process had to be augmented by the associative or intermediate stage of learning in which skill refinement takes place (Fitts, 1992). Attainment of these more advanced levels was built upon initial skill understanding and progressed via practice and repetition. Movements produced by the participants observably became more consistent moving towards an autonomous level. As participants were instructed on an exercise, their ability to perform the movement without trial strategies or complexity of thought became more apparent. This process occurred at various speeds for individuals and requested movements. Simpler movements such as a jog seemed to take far less time to learn while more complex or unfamiliar movements required more time to reach the associative stage. Evidence of achieving this stage was marked by the participants asking fewer questions about the 
exercise, their ability to hold conversations while performing the task, and fewer corrective cues needed by the instructor.

The basis of this three-part skill acquisition is the epicenter within the concept of using the aquatic environment as a gateway to other forms of exercise. The aquatic environment allows the participant to conceptualize the movement while the properties of the water slow their movements allowing for greater thought and provisional support in the instance of movement strategy error. It provides greater proprioceptive feedback and kinesthetic awareness to stimulate the learners' ability to develop an accurate internal movement reference (Adams, 1971). Water also allows for repetitive practice of a movement without physiological complications regarding compromised joints, chronic pain, or impaired balance. This could be of particular importance in reference to older adults obtaining a skill without obtaining an injury.

In relation to these clinical implications, all of the focus group participants made at least one comment pertaining to improvements in their functional abilities via advances in balance, ambulation, endurance, or flexibility. Many participants stated that their post-intervention exercise intensions included adoption of a past exercise that now felt easier or less painful to perform, while there was also mention of incorporating a new form of training. This elicits the notion of a learned skill resulting in improved movement-related self-efficacy and movement safety, as well as the use of the aquatic environment as a stepping-stone for continued exercise advancement.

Self-Imposed Ageism. An interesting caveat to the focus group findings was that of an almost self-imposed ageism. Participants readily brought up their age with the connotation that it was an accepted fact that age results in a state of disability. There was an impending sense of pride in the comments regarding their age when referencing their ability to participate in physical 
activity. This accepted social norm could be the crux of the problem with the lacking

participation in physical activity levels. Aging individuals are provided the outlook, the norm, of becoming more sedentary with age; thus they adhere to this expectation, or lack thereof (Hehman \& Bugental, 2013; O'Rourke \& Ceci, 2013). It may be that this is the starting point in which exercise interventions for aging adults must take place.

Changing the social norm via education may be the necessary starting point on the path towards a more physically active aging population. If older adults are not confronted with, or impose upon themselves, the notion that they are supposed to become less active as they age or that disability is impending with advancing age, then maybe a greater portion of the older adult population would challenge themselves to participate in exercise. Interestingly, Canada is already pressing this issue with the Active Living Coalition for Older Adults highlighting the topic in their report entitled: Overcoming Ageism in Active Living (O'Brien Cousins, 2005). Ageism, prevalent in both the U.S. and Canada, is being charged as the source of faded resolve to participate in physical activity and other associated areas of life (O'Brien Cousins, 2005). Topics including "conservation of the body," "acting their age," and "exertion is dangerous" were highlighted as ageist remarks that permeated older adults beliefs and actions $\left(\mathrm{O}^{\prime} \mathrm{Brien}\right.$ Cousins, 2005).

Ageism in Exercise Instruction. In addition to societal beliefs and norms impeding older adults participation in physical activity, fitness instructors can obtain some of the blame. The prevalence of surveying participants for safety and catering to specified needs in fitness instruction and leadership is noteworthy and important, but the means of implementing this appraisal should be reconsidered. Safety is a primary concern for all participants and should be reflected in exercise instruction for all participants. Directing isolated comments or concerns at 
older adults can impart a level of discomfort or insult to older adults participating in a group fitness environment. These types of ageism-oriented comments impart or reinforce an older adult's belief that maybe they shouldn't be participating in exercise.

One comment provided by a focus group attendee reinforces the belief that ageism in society has become an expectation: "I have to say too that in regard to the students and Lori [the instructor]. I did not hear sweetie, dearie - you know, that kind of thing. They treated us as people - not as little old people [participant laughs].” This statement also encourages the belief that instructional tone could enhance or hinder group fitness participation.

Implications for practice denoted from these findings and supporting literature suggest a need for both local and national educational campaigns to refute self-imposed ageism. Locally, additions to fitness instructor education to include information pertaining to ageism could be paramount for participation inclusivity. Nationally, there is a need to thwart the impression that age results in impending disability and inactivity via a public media campaign, in an attempt to shift the paradigm of "I can't" to "I can" in the aging population.

Initiation versus Continuation. Though the importance of utilizing the aquatic environment as a gateway to exercise participation is paramount, motivation to initiate exercise has to occur prior to any action being taken. The utilization of adult education strategies to impart awareness and motivation towards adopting physical activity could prove to be a powerful tool for initialization of exercise. It can serve the role of enlightening individuals on the importance, benefits, methods, and by what means to participate in physical activity. The source and delivery of the educational component are of additional importance when pertaining to adult learners. Many adult learning theorists believe that adult learning is rooted in a wealth of past experiences, self-direction, personal needs and interests, usefulness of the information or 
skill, and the amount of onus they can link to the experience (Knowles M.S. et al., 2011). If these components can be built in to the exercise education experience, this may result in greater physiological and cognitive improvement as well as initiation of exercise.

Physicians as Initiators. One of the primary opportunities for the recommendation of exercise participation is by means of a physician. Adults in the U.S. over the age of 65 visit their physician an average of eight times per year with an estimated $82-94 \%$ of the adult population making contact with their health care professional each year (Robinson, White, Jr., \& Houchins, 2006; CDC, 2014; Schutzer \& Graves, 2004). As these statistics illustrate, physicians have access to the vast majority of the aging population in addition to their undivided attention. The literature also supports that sedentary older adults have a greater instance of exercise initiation when the recommendation comes from their health care provider (Burton et al., 1999; Glasgow, Eakin, Fisher, Bacak, \& Brownson, 2001; Cohen-Mansfield et al., 2006; Schutzer \& Graves, 2004). This information highlights an educational opportunity from a perceptually powerful source for physical activity initiation.

Though a commanding argument, few physicians allocate time to provide exercise recommendations or education for older adults (Barnes \& Schoenborn, 2012). This is thought to be attributed to lack of time during the office visit, limited compensation for preventative counseling, and deficiency of physician training and perceived value as a behavioral counselor (Balde, Figueras, Hawking, \& Miller, 2003). This advances the researcher to believe that this wasted opportunity should be reproached via providing a behavior counselor educated on exercise implication, availability, and benefits to meet with patients during their office visits. Providing such a service may improve the adoption of physical activity by aging adults. 
Exercise Maintenance. It has been established that initiation of exercise is construed as being less challenging than maintaining the behavior of exercise (McAuley et al., 1993; Mullen et al., 2013). As previously discussed, the researcher believes that the individuals that volunteered for this study were at a heightened state of readiness, as evident by their action of joining the study. Their initiation of the program was probably a bit more challenging than that of a 'normal' exercise program in that they had to attend three assessment visits compounded over the course of three weeks leading up to the actual onset of exercise. During this assessment period, two of the participants dropped out of the study while one was asked not to continue due to language barriers that would adversely affect the cognitive testing parameters. Throughout the exercise intervention, two additional participants selected to discontinue participation due to personal choices while the other four subjects that dropped out did so in accordance to their physician's orders.

The low occurrence of self-selected attrition encourages the researcher to believe that the aquatic exercise program provided ample physiological stimulus to allow for challenge while eliciting a feeling of success (McAuley et al., 2000). The incorporation of adult education and progressive overload provided a step-wise advancement in the exercise sessions while integrating education for reasoning behind the exercises being performed. In addition to the low attrition rate, an average $91 \%(\mathrm{SD}=2.85)$ attendance was recorded, which attests to the participants' commitment to the intervention. Qualitative findings endorse that the participants were not only attending regularly due to their commitment, but they were also attending due to enjoyment and perceived physical improvement. Upon completion of the program, intervention participants in the focus group asked if they would continue with exercise: approximately $87 \%$ responded "yes." Not only did these individuals say that they would continue with some variety 
of exercise, they had specified plans for program continuation, many of whom wished to continue with some form of aquatic training.

When examining both qualitative and quantitative measures of the program in reference to exercise continuation, the findings lead the researcher to believe that the combination of adult education and the development of a safe learning environment, progressive overload, and the inherent characteristics of the aquatic environment fostered exercise continuation. Each of these components added value to the progression of the intervention, and ultimately, the intended continuation of exercise. The researcher proposes employing these variables to exercise programming for older adults to promote greater exercise success.

The ultimate goal is to convert sedentary, non-exercisers to physically active, chronic exercisers. Without continuation of habitual exercise participation, benefits resulting from exercise will diminish. Thus, it is imperative to build a program that promotes the individual's belief that exercise is important, achievable, accessible, and promotes health and wellness parameters central to their lifestyles.

Spatial Working Memory and Novice Exercisers. Findings from the present study promote the improvement of SWM in conjunction with movement economy via planning behaviors, goal selection, and execution (Badcock et al., 2005). Implications from this finding in combination with the literature support that novice exercisers may experience the most movement adaptation via aquatic exercise in accordance with SWM. During the early processes of skill acquisition, SWM is credited with contributing to visuomotor adaptation (Anguera et al., 2010; Anguera et al., 2011). Visuomotor adaptation effects how individuals interact with their environment and is believed to occur through sensorimotor alterations (Anguera et al., 2010; Anguera et al., 2011). Challenging the body to produce new movements and, in doing so, 
experience errors while deriving correction strategies and updating their understanding of movement space permits individuals to improve their SWM (Anguera et al., 2010; Anguera et al., 2011).

Sedentary individuals and novice exercisers may have a less diverse movement library resulting in an inefficiency to produce new or unfamiliar movements (Suetterlin \& Sayer, 2013). In direct relation to this, the lack of or loss of kinesthetic awareness is thought to be further stimulated by the aging process in accordance with reductions in other cognitive strategies such as movement processing speed and attention (Park \& Reuter-Lorenz, 2009). The culmination of movement strategy via visuomotor and sensorimotor adaptation may assist in the diversification of proprioceptive acuity.

The aquatic environments' unique attributes may aid in the ability of older adults to further develop SWM. The properties of the aquatic environment, buoyancy and viscosity in particular, naturally slow the movements down from what is normally experienced on land. This could allow for a more complete thought process of the performance, allowance of error strategy formulation, greater processing time for visuospatial information, and expansion of sensorimotor or kinesthetic awareness. Figure 5.2 summarizes the findings and how they interact with one another to expand and advance participation in physical activity with the integration of aquatic exercise. 
Figure 5.2: Continuation of Expansion with Exercise

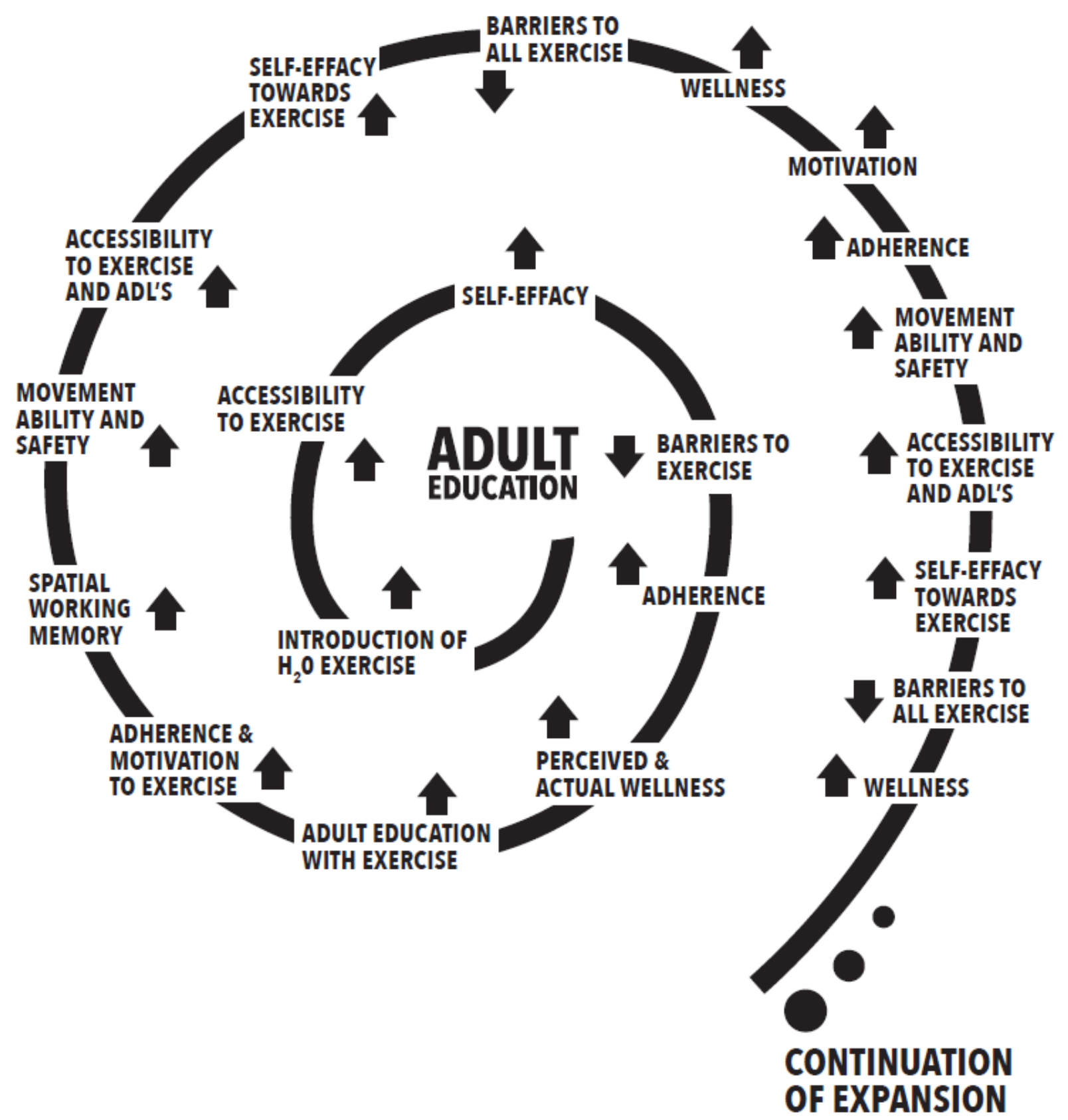

\section{Recommendations for Further Research}

Future research is necessary to elaborate and further justify the findings, or lack thereof, from this study. Due to the novelty of the aquatic environment and the lack of statistically significant results pertaining to executive function, the need for researching specified cognitive 
parameters in combination with aquatic exercise should be explored. It is evident from the qualitative remarks that the aquatic environment did positively affect participants' ability to take part in exercise. This could allow for greater exercise accessibility for aging adults, particularly those with physical limitations or low ability-related self-efficacy. However, confirmation that aquatic-based exercise can yield similar cognitive-related results to land-based training is needed in order to fully promote the use of aquatic exercise. Yes, an improvement in global wellness is an important factor, but cognitive enhancement is also of great concern for improved quality of life.

Procedural Alterations. Procedural alterations in research design as well as multiple formats of executive function testing should be considered. Of particular importance, considerations for frequency and duration of aquatic training as proposed by Denkinger (2012) in relation to the ACSM guidelines for older adults should be reexamined (Farrell, 2011). The current study utilized a 10-week aquatic intervention that met 3-days for 60 minutes each session. Studies support using a minimum of 8-10 week duration intervention for measurable physiologic changes (Becker, 2009). Future research warrants a study that compares the cognitive improvements gleaned over the course of a 10 -week exercise intervention with a control/non-exercise group, 5-day x 30 minute exercise group, and 3-day x 50 minute exercise group. This prescribed study could assist in defining a standard for exercise prescription in relation to optimal executive function gains through an exercise program. Both outcome and participant attendance should be monitored in order to determine best practices for cognitive function improvements. This is necessary to determine if individuals are more willing to participate in one exercise scheme over the other. Continued involvement in exercise training is 
paramount thus, determining if individuals would be willing to participate in exercise 5-days per week in contrast with 3-days per week is essential.

Duration. Duration of the exercise intervention should also be considered for further study. The current study assessed a 10-week intervention period but other duration intervals should be researched to evaluate both optimal gains in physiological as well as cognitive parameters when performing aquatic exercise. The literature covers the gamut of acute (a single bout) through chronic effects ( $\geq 3$ months) of exercise on both parameters for land-based training. A similar body of literature needs to be developed for the aquatic environment. Assessing various parameters of cognitive function and physiological adaptation is desirable to promote the use of aquatic exercise for global improvement in both variables.

Executive Function Assessment. Additionally, cognitive parameters should be evaluated with a variety of assessments to ensure validity of the results and to have a reserve method in place in the event of technical difficulties, such as those experienced during RTI testing in the current study. A litany of assessments for various elements of executive function have been validated. The CANTAB instrument was selected due to the professed sensitivity to diverse alterations in cognitive function. The researcher should have recognized that technical complications could transpire to alter the outcomes of the testing battery. Thus, for future studies, it would be recommended to include comparable tests to the battery being utilized. In conjunction with this, it would be wise to implement fewer cognitive tests to assist in the prevention of responder fatigue and assure a more accurate outcome. Assessments including Towers of London or Tower of Hanoi, Wisconsin Card Sorting Test, Sustained Attention to Response Task, Six Elements Test, Greenwich Test, or Multiple Errands Tests could also be 
employed that mirror tests from the CANTAB assessments (Chan, Shum, Toulopoulou, \& Chen, 2008).

Land vs. Aquatic. Future research concerning executive function fortification and advancement should be addressed comparing the effects of land and aquatic environments. The current study did not employ an analogous land-based exercise group to compare to the aquaticbased group. Comparison of the groups may be an effective means of deliberating the effects of exercise type on cognitive function. Additionally, utilizing a similar research design as employed in this study with the addition of a similar land-based exercise group could address questions concerning attrition and participation, psychosocial mediators--with special interest in barriers and self-efficacy as well as fitness and wellness.

Psychosocial Mediators. Implementation of a control participant focus group may assist in a more complete understanding of why psychosocial mediators remained so high throughout the study without participation in the intervention. Employing focus groups for both the control and intervention groups may yield interesting insights into changes, or lack thereof, in psychosocial mediator variables. Asking questions pertaining to social support, self-efficacy, barriers, and motivators may provide further information on how these variables change throughout the course of an intervention period. It may also be beneficial to determine the stage of change that the participants are in prior to and following the intervention period and relate this to the psychosocial mediators. Findings from this type of study may reveal connections to stages of change, psychosocial mediators, and projected participation in exercise.

Diversify Population Recruitment. The population in the study did not have clinically diagnosed cognitive impairment. The lack of impairment may have stunted the cognitive function improvements illustrated in this study. It may be that the lack of clinically impaired 
cognitive deficiency relegates the participants to the highest end of the cognitive function spectrum creating a virtual ceiling effect for current executive function assessments. Many studies in the literature base and validated assessments cater to individuals with mild to severe cognitive impairments. Studies commonly remark on the detail that individuals with greater impairment show greater improvement as a response to exercise. Diversifying the populations recruited for aquatic-based studies may assist in clarifying cognitive advantages garnered from aquatic exercise. Recruitment strategies would be similar in nature to the present study with the inclusion of individuals with mild cognitive impairment as well as severe cognitive impairment. One additional recruitment tactic would be the inclusion of working with physicians that specialize in cognitive function. This would allow the researcher to have greater access to the necessary population while providing an outlet for exercise to the patients. Performing research comparing non-impaired, mildly impaired, and severely-impaired individual's cognitive function changes during the course of an aquatic exercise intervention could provide a more complete understanding of how aquatic exercise affects executive function.

Follow-Up Assessments. The researcher recommends a 6 month follow-up on the current intervention participants to address physical activity status. Questions pertaining to level of physical activity, type of exercise participation, as well as duration and intensity of exercise involvement should be addressed. Inquiry on the individual's level of function along with their ability to complete their ADLs would also be researched. Lastly, modifications in health history including alterations in medical conditions and changes in medication dosage or usage would be investigated.

Advancing Aquatic Literature. A more solid body of literature in relation to the aquatic environment needs to be established. Though there are some foundational studies that 
provide an operational base knowledge for clinical practices and research, there is a litany of topics that need exploration. Research concerning the effects of the water temperature on hunger response, with particular focus on insulin and leptin response should also be conducted. Findings from such research could have a major impact on weight loss intervention programs that utilize aquatic exercise as the primary exercise medium. Further, findings might help to explain the results of the current study pertaining to the lack of weight loss or body composition alterations. In addition, studies concerning the effects of water immersion on beta-blocker response in relation to exercising heart rate must be examined. Reconfiguration of the Kruel deduction for aquatic target heart rates may need to be considered for this population beyond the land-standard of subtracting $20 \mathrm{bpm}$. This could have a major impact on training response with aquatic exercise, yielding superior advancements in cardiovascular fitness.

Aquatic Exercise as a Gateway. Further study investigating the use of aquatic exercise as a gateway exercise should be explored. Aquatic exercise provides a more inclusive environment for participation allowing for greater accessibility of exercise to various populations. The effects of the aquatic environment as a medium for pre-training, improved ability-related self-efficacy, and enhanced biomechanics in relation to exercise could pose answers to how to make exercise accessible to all. To assess this theory, research employing known barriers to exercise participation should be individually and jointly studied in relation to aquatic exercise. Empirically supported barriers in areas of health status (e.g., pain, balance, endurance, strength, injury, etc.), environment (availability and safety for exercise site), and knowledge (role of exercise, health benefits, etc.) should be the preliminary research concern (Schutzer \& Graves, 2004). 
Studies concerning a known barrier and how that barrier is perceived throughout an aquatic exercise intervention could provide considerable insight into positively altering exercise accessibility. For example, employing a study testing the effects of an aquatic exercise intervention on balance parameters in relation to exercise participation probability, physiological modifications, and effects on global physical activity as well as quality of life could provide valuable information on how to disrupt a sedentary lifestyle pattern in the aging adult.

Retirement Intervention Program. This study found that the lifestyle change of retirement was accompanied by a lifestyle shift in activity level. This finding has also been supported by the literature (Barnett, van, Ogilvie, \& Wareham, 2014; Chung, Domino, Stearns, \& Popkin, 2009; Barnett, van Sluijs, \& Ogilvie, 2012). With this known and planned lifestyle alteration, the researcher recommends investigating the potential for a retirement-focused exercise program implementation. With the major lifestyle shift and initial perceived availability of time, introducing an exercise program (land or aquatic) immediately following retirement may improve physical activity levels in the aging adult. Behavioral modifications and global physical activity levels should be studied with the inclusion of exercise exit programs for retirees.

Physician Education Directive. It has been noted in the literature that individuals that receive education or recommendations pertaining to exercise from a health care provider are more likely to initiate an exercise behavior (Burton et al., 1999; Glasgow et al., 2001; CohenMansfield et al., 2006; Schutzer \& Graves, 2004). A study implementing exercise education or prescription to older adults during a physician's office visit with physical activity tracking should be considered. Employing behavioral counselors knowledgeable on the topic of exercise to educate or make recommendations to older adults during physician visits could provide an influential means of exercise initiation. A preliminary physical activity questionnaire could 
assist in determining baseline levels while tracking physical activity via a pedometer, electronic device (mobile app or activity tracker band), or phone check-ups. Additionally, various adulteducation tactics (e.g., aligning reason for exercise with the adult's needs, providing options, linking to past experiences) should be employed to affirm the most effective educational strategy.

Alterations in Spatial Working Memory. One of the implications of this study is that SWM may experience greater alterations and advancements in novice exercisers and sedentary individuals. Findings from an experimental design that employed a pre- and post-test (assessment) of SWM across participants of varied baseline levels of exercise experience could be useful towards confirming this implication. Additionally, comparison of land versus water training may also be of value in SWM adaptation. The researcher speculated that the aquatic environment slows down movement and provides a setting for consequence-free movement, which assisted in the further adaptation of SWM. This theory should be tested via a land-aquatic comparison study.

Effects of Adult Education on Exercise Initiation. Lastly, a study that examined the effects of adult education on exercise in accordance with known barriers and motivators could provide useful information for enhancing exercise participation in the older adult population. Studies focusing on adult education should be concerned with two primary categories: initiation and continuation. Studies providing adult education as a stimulus for adopting exercise should examine the following: What content/information is most effective? What strategies and locations are optimal for the provision of education? At what age(s) are older adults most open to the suggestion of exercise? Additionally, studies are needed that examine different methods of creating a positive learning environment. These studies should incorporate education on the 
'why' of exercise and align with the needs and wants of participants as they pertain to exercise.

These studies should also allow for and examine self-selected individualization of exercise experience. 


\section{Appendix}

$\begin{array}{ll}\text { Appendix A: Informed Consent } & 205\end{array}$

Appendix B: Phone Transcript 210

Appendix C: Health History \& Demographic Data 212

Appendix D: Aquatic Program Design 216

$\begin{array}{ll}\text { Appendix E: Anthropometric Measurements } & 218\end{array}$

Appendix F: Psychosocial Mediator Surveys 219

Appendix G: 6-Minute Walk Test 222

$\begin{array}{ll}\text { Appendix H: Focus Group } & 224\end{array}$

Appendix I: Focus Group Transcripts 227

Appendix J: Triangulation Matrix for RQ1 257

Appendix K: Triangulation Matrix for RQ2 \& RQ3 259

Appendix L: Triangulation Matrix for RQ4 261

$\begin{array}{ll}\text { Appendix M: Matched Pairs } & 270\end{array}$ 


\section{Appendix A: Informed Consent}

\section{WestVirginiaUniversity}

Human Research Protocol Only Minimal RiskConsentEorm

(With HIPAA)

\section{Only Minimal Risk \\ Consent Information and HIPAA Form}

Principal Investigator

Department

Protocol Number

Study Title

Co-Investigator(s)

Sponsor (if any)

Performance and Applied Exercise Science

1306049622
Lori A. Sherlock, M.S.

Human Performance \& Applied Exercise Science

The effects of Aquatic Exercise on Executive Function

James Rye, Ph.D.

West Virginia University, College of Human Resources and Education \& Human

\section{Contact Persons}

In the event you experience any side effects or injury related to this research, you should contact Lori Sherlock at (304) 293-6509 (After hours contact: Lori Sherlock at (304) 685-4649). If you have any questions, concerns, or complaints about this research, you can contact Lori Sherlock at 304-293-6509.

For information regarding your rights as a research subject, to discuss problems, concerns, or suggestions related to the research, to obtain information or offer input about the research, contact the Office of Research Compliance at (304) $293-7073$.

In addition if you would like to discuss problems, concerns, have suggestions related to research, or would like to offer input about the research, contact the Office of Research Integrity and Compliance at 304-293-7073.

\section{Introduction}

You, have been asked to participate in this research study, which has been explained to you by Lori Sherlock. This study is being conducted by Lori Sherlock and James Rye, Ph.D. in the Department of Human Performance and Applied Exercise Science and the Department of Human Resources and Education \& the Department of Education at West Virginia University.

\section{Purpose(s) of the Study}

The purpose of this study is to learn more about the effects of aquatic exercise on cognitive (mental processes) function. WVU expects to enroll approximately $30-40$ participants for this study.

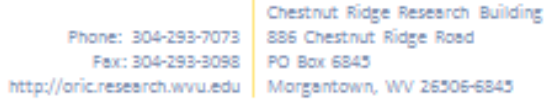

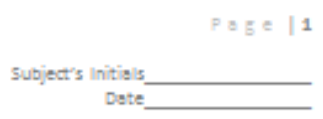




\section{Description of Procedures}

This study involves 3, 1-hour, assessment visits followed by a 10-week aquatic group fitness class that will meet on Monday, Wednesday and Friday for 1 hour each (3 hours/week). After the 10-weeks of exercise, there will be a focus group, lasting approximately 1 hour, and 2 assessment visits, lasting around 1 hour each. In total the study will take 14 weeks for you to complete. Participants will be randomly (like flipping a coin) assigned after the initial visits to exercise group or control group. The control group will receive the aquatic exercise program following the conclusion of the study. All participants will need to obtain physician clearance from their personal physician.

During the first visit, the consent form will be reviewed and then you will be asked to become familiar with a computer test on a touch-screen computer, complete a health history, demographic and physical activity interview. At the second visit, you will have height, weight, hip-to-waist, and body composition, blood pressure, and heart rate measurements taken. You will be asked to fill out questionnaire regarding barriers, social support, and self-efficacy (the ability to achieve a desired result) related to exercise. You do not have to answer all the questions. You will have the opportunity to see the questionnaire before signing this consent form. You will also complete a computerized cognitive screening test. Visit 1 and 2 should take approximately 1 hour each. The third visit you will complete a 6 -minute walk test on a flat, indoor area and should take no longer than 30 minutes.

After the preliminary assessments, you will take part in a 10-week aquatic exercise class that will meet on Monday, Wednesday and Friday for 1 hour each day, totaling 3 hours a week for 10 weeks. During week 5 of the aquatic exercise classes, you will be asked to repeat the same questionnaire regarding barriers, social support, and self-efficacy related to exercise that you completed during your initial assessment. You do not have to answer all the questions. During your final week of the exercise classes, you will be asked to participate in a focus group that will meet after the class for approximately 1 hour. The focus group will be audiotaped.

Following the 10-week aquatic exercise class, you will have height, weight, hip-to-waist, and body composition, blood pressure, and heart rate measurements taken, as well as note any changes in your health history. You will be asked to fill out the same questionnaire regarding barriers, social support, and self-efficacy related to exercise. You do not have to answer all the questions. You will also complete a computerized cognitive screening test. This visit should take around 1 hour to complete. Lastly, you will take part in another 6-minute walk test. The final visit should take no longer than 30 minutes. Participants will be asked to react confidentially to emerging themes collected during the focus group. In signing this, you are agreeing not to change your physical activity status until you are assigned to the treatment group, in which case you will participate in the aquatic exercise classes provided.

\section{Discomforts}

There are no known or expected risks from participating in this study, except for the mild frustration associated with answering the questions and the minimal risk of injury during the exercise test and aquatic exercise sessions. In rare cases these exertions can lead to chest pain, shortness of breath, irregular heartbeats and dizziness. During exercise there is a transient (existing briefly) increased risk of having a cardiac related complication, however, risk is extremely small. If you decide not to participate in this study it will not affect your employment or student status at WVU.

\section{Benefits}

You may not receive any direct benefit from this study. You will receive valuable health-related information as well as 10 weeks of professionally instructed aquatic aerobics classes. You may experience positive effects in cognitive function

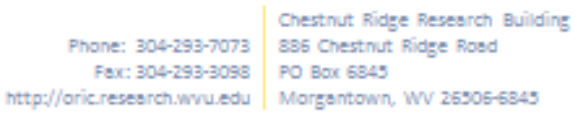

inttp://oricresearch.wvu.edu Morgentown, WV 25905-6849
$P \& g \in 12$

Subject's initials

Date 
and cardiovascular health. Aside from this, there may be no direct benefit for being in this study. Your participation in this study may eventually benefit others. The results of the assessments will be made available to you, and with your consent only, to your personal physician. You and your physician may find this information of value in their healthrelated recommendations to you.

\section{Financial Considerations}

If you are injured as a result of this research, treatment will be available. Responsibility for this treatment will be borne by you. In the event that you are physically injured as a result of participating in this research, care will be available. You will, however, be responsible for the charges for the care. There is no commitment to provide any compensation for research-related injury. You should realize, however, that you have not released this institution from liability for negligence. Please contact the investigator, Lori Sherlock at 304-293-6509 if you are injured or for further information.

\section{Confidentiality}

Any information about you that is obtained as a result of your participation in this research will be kept as confidential as legally possible. Your research records and test results, just like hospital records, may be subpoenaed by court order or may be inspected by the study sponsor or federal regulatory authorities without your additional consent.

Audiotapes or videotapes will be kept locked up and will be destroyed as soon as possible after the research is finished. In any publications that result from this research, neither your name nor any information from which you might be identified will be published without your consent.

\section{HIPAA}

We know that information about you and your health is private. We are dedicated to protecting the privacy of that information. Because of this promise, we must get your written authorization (permission) before we may use or disclose your protected health information or share it with others for research purposes.

You can decide to sign or not to sign this authorization section. However, if you choose not to sign this authorization, you will not be able to take part in the research study. Whatever choice you make about this research study will not have an effect on your access to medical care.

\section{Persons/Organizations Providing the Information}

The research site carrying out this study will be providing the information. This includes WVU College of Human Resources and Education (James Rye, Ph.D.) and the Department of Human Performance and Applied Exercise Science (Lori A. Sherlock, M.S.).

\section{Persons/Organizations Receiving the Information}

The research site receiving the information from this study includes WVU College of Human Resources and Education (James Rye, Ph.D.) and the Department of Human Performance and Applied Exercise Science (Lori A. Sherlock, M.S.).

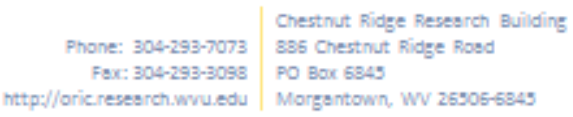

\author{
nttp://oricreseserch.wvu.edu Morgentown. WV 26905-5949
}

$P=g \in \mid 3$

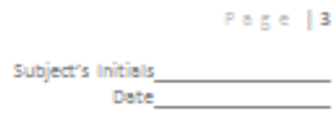




\section{The Following Information Will Be Used}

New information that is created or collected during the study such as: health history and physical assessments, visit notes, staff notes, demographic data, and study forms will be used for comparison and association to the relation of exercise and cognitive function.

\section{The Information is Being Disclosed for the Following Reasons}

- Review of your data for quality assurance purposes

- Publication of study results (without identifying you)

- Other research purposes such as reviewing the safety or effectiveness of the study therapies; evaluating

therapies for patients; developing a better understanding of cognitive function; improving the design of future exercise protocols

\section{You May Cancel this Authorization at Any Time by Writing to the Principal Investigator}

Lori A. Sherlock

PO Box 2227

Morgantown, WV 26506

If you cancel this authorization, any information that was collected already for this study cannot be withdrawn. Once information is disclosed, according to this authorization, the recipient may redisclose it and then the information may no longer be protected by federal regulations.

You have a right to see and make copies of your medical records. You will not be able to see or copy your records related to the study until the sponsor has completed all work related to the study. At that time you may ask to see the study doctor's files related to your participation in the study and have the study doctor correct any information about you that is wrong.

This authorization will expire at the end of the study unless you cancel it before that time.

\section{Voluntary Participation}

Participation in this study is voluntary. You are free to withdraw your consent to participate in this study at any time. Refusal to participate or withdrawal will not affect your future care, your employee status at WVU and will involve no penalty to you.

In the event new information becomes available that may affect your willingness to participate in this study, this information will be given to you so that you can make an informed decision about whether or not to continue your participation.

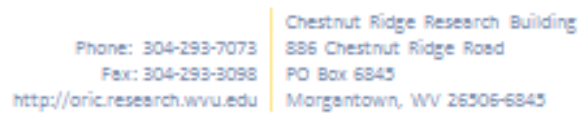

intp://oric.reseserch.wwu.edu Morgentown, Wv 26905-6945

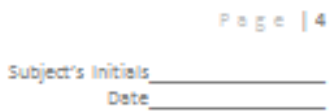




\section{WWestVirginiaUniversity.}

Human Research Protocol Only Minimal RiskConsentForm

(With HIPAA)

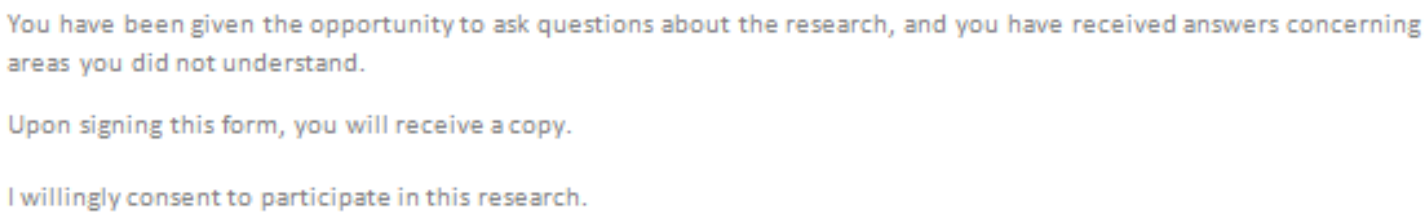

\section{Signatures}

Signature of Subject

\begin{tabular}{lll}
\hline Printed Name & Date & Time
\end{tabular}

Please indicate if you would like the incidental findings to be shared with you following the study.

NO

The participant has had the opportunity to have questions addressed. The participant willingly agrees to be in the study.

Signature of Investigator or Co-Investigator

$\begin{array}{lll}\text { Printed Name } & \text { Date }\end{array}$

$P a g e \mid 5$

Phone: 304-293-7073 895 Chertnut Ridge Roed

Fax: 304-293-3098 PO Box 6849

nttp://oricresearch.wvu.edu | Morgentown, WV 26905-6949

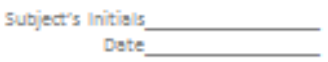




\section{Phone Transcript:}

Appendix B: Phone Transcript

Thank you for calling to inquire about the water exercise and cognitive function research study. Is it okay if I ask you a few questions to determine if you are eligible for the study?

Are you between the ages of 60-90? YES NO

Do you have any known neurological defect such as dementia, multiple sclerosis, brain tumor, or Parkinson's disease? YES NO

Are you willing and able to participate in a regular, water aerobic exercise program?

YES NO

Are you currently participating in regular exercise training for more than 30 minutes 3 times per week? YES NO

Would you be willing to obtain a physician's release to participate in an exercise program?

YES NO

Do you have one or more of the following conditions?

-open, draining wounds that cannot be covered by an occlusive dressing YES NO -severe burns YES NO

-fever, infection, or severely compromised immune function YES NO

-bowel or bladder incontinence YES NO

-cardiac failure or unstable blood pressure YES NO

-Deep Vein Thrombosis YES NO

-Uncontrolled Epilepsy YES NO

-Renal Disease or dysfunction YES NO

-severe or contagious dermatological disorder YES NO

-non-tunnel catheter YES NO

-respiratory disease with a $\mathrm{VC}<1500$ YES NO

If the potential participant meets the eligibility requirements read:

The answers you provided suggest that you are eligible to participate in this research study. If you are interested, I would like to set up an appointment for you to come to the Human Performance Lab on the ground floor of the Health Science Center to collect the preliminary information prior to you beginning the exercise program. When you come for your first visit, we will be collecting demographic data, anthropometric measurements including height, weight, hip-to-waist ratio and body fat assessment using the Bod Pod, completion of a lifestyle questionnaire (past year total physical activity questionnaire), and completion of a health history. Cognitive screening using the mini mental state examination will also be conducted to assess orientation, registration, attention, calculation, language and recall. We would also like to collect 
your medical release provided by your personal physician. I can send this form to you via mail or e-mail. Which would you prefer? MAIL E-MAIL

Name:

Street Address:

City/State/Zip:

E-Mail Address

OR

Date/Time of Appointment:

If the potential participant does NOT meet the eligibility requirements read:

Thank you for your interest in participating in this research study, however, you currently do not meet the eligibility requirements. Thank you for your time. 
Appendix C: Health History and Demographic Data Form

\title{
Health History \& Demographic Form
}

\author{
Participant Demographic Information
}

Participant \#:

Sex: Male

Female

Marital Status: $\mathrm{M}$

$\mathrm{S}$

W

$\mathrm{D}$

Birthdate:

Please circle the highest grade in school you have completed:

$\begin{array}{lllllllll}\text { Elementary School } & 1 & 2 & 3 & 4 & 5 & 6 & 7 & 8\end{array}$

$\begin{array}{lllll}\text { High School } & 9 & 10 & 11 & 12\end{array}$

$\begin{array}{llllllllll}\text { College/Postgrad } & 13 & 14 & 15 & 16 & 17 & 18 & 19 & 20+\end{array}$

Race or ethnic background:

White, not of Hispanic origin $\square$ American Indian/Alaskan native

Black, not of Hispanic origin $\square$ Pacific Islander

Asian

$\square$ Hispanic

What is your job or occupation? Check the one that applies to the greatest percent of your time.
Health professional
Disabled, unable to work
Service
Manager, educator, professional
Skilled crafts
Operator, fabricator, laborer
Unemployed
Technical, sales, support
$\square$ Homemaker
Retired
Student

Health History

Symptoms or Signs Suggestive of Disease

Place a check in the box if your answer is "yes."

1. Have you experienced unusual pain or discomfort in your chest, neck, jaw, arms, or other areas that may be due to heart problems?

2. Have you experienced unusual fatigue and/or shortness of breath at rest, during usual activities, or during mild-to-moderate exercise (e.g., climbing stairs, carrying groceries, brisk walking, cycling, etc.)?

3. Have you had any problems with dizziness or fainting?

4. When you stand up, or sometimes during the night while you are sleeping, do you have difficulty breathing?

5. Do you suffer from swelling of the ankles (ankle edema)?

6. Have you experienced an unusual and rapid throbbing or fluttering of the heart? 
7. Have you experienced severe pain in your leg muscles during walking?

8. Has a doctor told you that you have a heart murmur?

\section{Medical History}

Please check which of the following conditions you have had or now have. Also check medical conditions in your family (father, mother, brother(s), or sister(s)). Check as many as apply.

\section{Personal FamilyMedical Condition}

Coronary heart disease, heart attack, coronary artery surgery Angina

High blood pressure

Peripheral vascular disease

Phlebitis or emboli

Other heart problems (specify:

Lung cancer

Breast cancer

Prostate cancer

Colorectal cancer (bowel cancer)

Skin cancer

Other cancer (specify:

Stroke

Chronic obstructive pulmonary disease (emphysema)

Pneumonia

Asthma

Bronchitis

Diabetes mellitus

Thyroid problems

Kidney disease

Liver disease (cirrhosis of the liver)

Hepatitis (A, B, C, D, or E)

Gallstones/gallbladder disease

Osteoporosis

Arthritis

Gout

Anemia (low iron)

Bone fracture

Major injury to foot, leg, knee, hip, or shoulder

Major injury to back or neck

Stomach/duodenal ulcer

Rectal growth or bleeding

Cataracts

Glaucoma

Hearing loss 


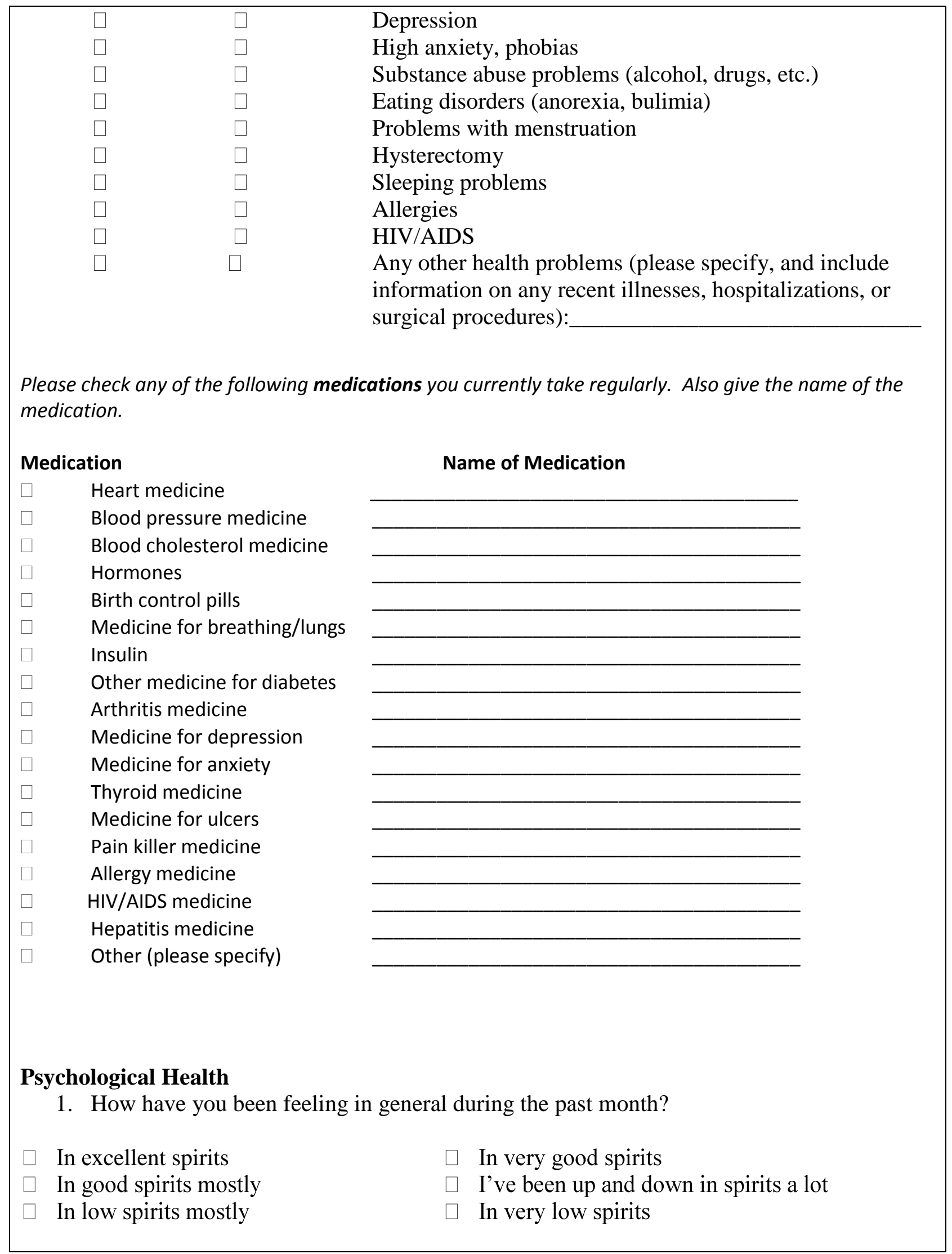


2. During the past month, would you say that you experienced a lot of $\quad \square$ moderate $\quad \square$ relatively little $\quad \square$ almost none stress?

3. In the past year, how much effect has stress had on your health:
a lot
some
hardly any or none

4. On average, how many hours of sleep do you get in a 24-hour period?

Less than $5 \quad \square \quad 5$ to $6.9 \quad \square 7$ to $9 \quad \square$ More than 9

\section{Substance Use}

1. Have you smoked at least 100 cigarettes in your entire life?
Yes
No

2. How would you describe your cigarette smoking habits?

Never smoked $\quad \square$ Used to smoke

How many years has it been since you smoked? years

Still smoke

How many cigarettes a day do you smoke on average? cigarettes/day

3. How many alcoholic drinks do you consume? (A "drink" is a glass of wine, a wine cooler, a bottle/can of beer, a shot glass of liquor, or a mixed drink).
Never use alcohol
Less than 1 per week
1 to 6 per week
1 per day
2 to 3 per day
More than 3 per day

REFERENCES USED IN FORMULATING QUESTIONNAIRE

1. National Center for Health Statistics, Stephens T and Schoenborn C. 1988. Adult health practices in the United States and Canada. Vital and Health Statistics. Series 5, No. 3. DHHS Public Health Service. Washington: U.S. Government Print Office.

2. Benson V and Marano MA. Current estimates from the National Health Interview Survey, 1993. National Center for Health Statistics. Vital Health Stat 10(190), 1994.

3. American School Health Association, Association for the Advancement of Health Education, Society for Public Health Education, Inc. The National Adolescent Student Health Survey: A Report on the Health of America's Youth. Oakland, CA: Third Party Publishing Company, 1989.

4. Nieman DC. Fitness and Sports Medicine: A Health-Related Approach. Palo Alto: Bull Publishing, 1995.

5. National Cancer Institute. DIETSYS Version 3.0, User's Guide: Health Habits and History Questionnaire. Bethesda, MD: National Cancer, Institute, 1993.

6. McDowell I and Newell C. Measuring Health: A Guide to Rating Scales and Questionnaires. New York: Oxford University Press, 1996.

7. American College of Sports Medicine. Guidelines for Graded Exercise Testing and Prescription. (5th edition). Philadelphia: Lea \& Febiger, 1995.

8. American College of Sports Medicine. Sol N and Foster C (eds). Health/Fitness Facility Standards and Guidelines. Champaign, IL: Human Kinetics Books, 1992.

9. U.S. Preventive Services Task Force. Guide to Clinical Preventive Services. (2nd edition). Alexandria, VA: International Medical Publishing, 1996. 
Appendix D: Aquatic Program Design

\section{Aquatic Exercise \& Cognitive Function Exercise Session Training Block}

\begin{tabular}{|c|c|c|c|}
\hline Session \# & Warm Up Duration & $\begin{array}{c}\text { Main Set Duration \& Intensity } \\
\% \text { are in HRR with Kruel } \\
\text { Modification Factor }\end{array}$ & Cool Down Duration \\
\hline 1 & $\begin{array}{l}10 \text { minutes } \\
\text { Building to } 40-50 \% \text { HRR }\end{array}$ & $\begin{array}{l}25 \text { minutes } \\
15 \text { min @ 45-50\% } \\
10 \text { min @ 50-60\% }\end{array}$ & 10 minutes \\
\hline 2 & $\begin{array}{l}10 \text { minutes } \\
\text { Building to } 40-50 \% \text { HRR }\end{array}$ & $\begin{array}{l}25 \text { minutes } \\
15 \text { min @ 45-50\% } \\
10 \text { min@ @ 50-60\% }\end{array}$ & 10 minutes \\
\hline 3 & $\begin{array}{l}10 \text { minutes } \\
\text { Building to } 40-50 \% \text { HRR }\end{array}$ & $\begin{array}{l}30 \text { minutes } \\
15 \text { min @ 45-50\% } \\
15 \text { min@ @ 50-60\% }\end{array}$ & 10 minutes \\
\hline 4 & $\begin{array}{l}10 \text { minutes } \\
\text { Building to } 40-50 \% \text { HRR }\end{array}$ & $\begin{array}{l}30 \text { minutes } \\
15 \text { min @ 45-50\% } \\
15 \text { min@50-60\% }\end{array}$ & 10 minutes \\
\hline 5 & $\begin{array}{l}10 \text { minutes } \\
\text { Building to } 40-50 \% \text { HRR }\end{array}$ & $\begin{array}{l}30 \text { minutes } \\
15 \text { min @ 45-50\% } \\
15 \text { min@ @ 50-60\% }\end{array}$ & 10 minutes \\
\hline 6 & $\begin{array}{l}10 \text { minutes } \\
\text { Building to } 40-50 \% \text { HRR }\end{array}$ & $\begin{array}{l}35 \text { minutes } \\
15 \text { min @ 45-50\% } \\
20 \min @ 50-60 \% \\
\end{array}$ & 10 minutes \\
\hline 7 & $\begin{array}{l}10 \text { minutes } \\
\text { Building to } 45-50 \% \text { HRR }\end{array}$ & $\begin{array}{l}35 \text { minutes } \\
15 \text { min @ 45-50\% } \\
20 \text { min@ @ 50-60\% }\end{array}$ & 10 minutes \\
\hline 8 & $\begin{array}{l}10 \text { minutes } \\
\text { Building to } 45-50 \% \text { HRR }\end{array}$ & $\begin{array}{l}35 \text { minutes } \\
25 \text { min @ 50-60\% } \\
10 \min @ 60-65 \% \\
\end{array}$ & 10 minutes \\
\hline 9 & $\begin{array}{l}10 \text { minutes } \\
\text { Building to } 45-50 \% \text { HRR }\end{array}$ & $\begin{array}{l}35 \text { minutes } \\
35 \text { min @ 55-65\% }\end{array}$ & 10 minutes \\
\hline 10 & $\begin{array}{l}10 \text { minutes } \\
\text { Building to } 45-50 \% \text { HRR }\end{array}$ & $\begin{array}{l}35 \text { minutes } \\
35 \text { min @ 60-65\% }\end{array}$ & 10 minutes \\
\hline 11 & $\begin{array}{l}\text { 5-7 minutes } \\
\text { Building to } 45-50 \% \text { HRR }\end{array}$ & $\begin{array}{l}35 \text { minutes } \\
35 \text { min @ 60-70\% }\end{array}$ & 10 minutes \\
\hline 12 & $\begin{array}{l}5-7 \text { minutes } \\
\text { Building to } 45-50 \% \text { HRR }\end{array}$ & $\begin{array}{l}40 \text { minutes } \\
40 \text { min @ 60-70\% }\end{array}$ & $5-7$ minutes \\
\hline 13 & $\begin{array}{l}5-7 \text { minutes } \\
\text { Building to } 50-60 \% \text { HRR }\end{array}$ & $\begin{array}{l}40 \text { minutes } \\
40 \text { min @ 60-70\% }\end{array}$ & 5-7 minutes \\
\hline 14 & $\begin{array}{l}5-7 \text { minutes } \\
\text { Building to } 50-60 \% \text { HRR }\end{array}$ & $\begin{array}{l}40 \text { minutes } \\
40 \text { min @ 65-70\% }\end{array}$ & $5-7$ minutes \\
\hline 15 & $\begin{array}{l}5-7 \text { minutes } \\
\text { Building to } 50-60 \% \text { HRR }\end{array}$ & $\begin{array}{l}45 \text { minutes } \\
15 \text { min @ 60-70\% } \\
30 \min @ 65-70 \% \\
\end{array}$ & 5-7 minutes \\
\hline 16 & $\begin{array}{l}5-7 \text { minutes } \\
\text { Building to } 50-60 \% \text { HRR }\end{array}$ & $\begin{array}{l}45 \text { minutes } \\
15 \text { min@60-70\% } \\
30 \text { min@65-70\% }\end{array}$ & $5-7$ minutes \\
\hline 17 & 5-7 minutes & 45 minutes & 5-7 minutes \\
\hline
\end{tabular}




\begin{tabular}{|c|c|c|c|}
\hline & Building to $55-60 \%$ HRR & $\begin{array}{l}15 \min @ 60-70 \% \\
30 \min @ 65-70 \%\end{array}$ & \\
\hline 18 & $\begin{array}{l}5-7 \text { minutes } \\
\text { Building to } 55-60 \% \text { HRR }\end{array}$ & $\begin{array}{l}45 \text { minutes } \\
5 \text { min @ 60-70\% } \\
40 \text { min @ 65-70\% }\end{array}$ & 5-7 minutes \\
\hline 19 & $\begin{array}{l}5-7 \text { minutes } \\
\text { Building to } 55-60 \% \text { HRR }\end{array}$ & $\begin{array}{l}45 \text { minutes } \\
45 \text { min @ 65-70\% }\end{array}$ & 5-7 minutes \\
\hline 20 & $\begin{array}{l}5-7 \text { minutes } \\
\text { Building to } 55-60 \% \text { HRR }\end{array}$ & $\begin{array}{l}45 \text { minutes } \\
45 \text { min @ 65-70\% } \\
\end{array}$ & 5-7 minutes \\
\hline 21 & $\begin{array}{l}5-7 \text { minutes } \\
\text { Building to } 55-60 \% \text { HRR }\end{array}$ & $\begin{array}{l}45 \text { minutes } \\
40 \text { min @ 65-70\% } \\
5 \text { min @ 70-75\% }\end{array}$ & 5-7 minutes \\
\hline 22 & $\begin{array}{l}5-7 \text { minutes } \\
\text { Building to } 55-60 \% \text { HRR }\end{array}$ & $\begin{array}{l}45 \text { minutes } \\
40 \text { min @ 65-70\% } \\
5 \text { min @ 70-75\% }\end{array}$ & 5-7 minutes \\
\hline 23 & $\begin{array}{l}5-7 \text { minutes } \\
\text { Building to } 55-60 \% \text { HRR }\end{array}$ & $\begin{array}{l}45 \text { minutes } \\
30 \text { min @ 65-70\% } \\
5 \text { min @ } 70-75 \% \\
5 \text { min @ 65-70\% } \\
5 \text { min @ 70-75\% }\end{array}$ & 5-7 minutes \\
\hline 24 & $\begin{array}{l}5-7 \text { minutes } \\
\text { Building to } 55-60 \% \text { HRR }\end{array}$ & $\begin{array}{l}45 \text { minutes } \\
30 \text { min @ 65-70\% } \\
5 \text { min @ 70-75\% } \\
5 \text { min @ 65-70\% } \\
5 \text { min @ 70-75\% }\end{array}$ & 5-7 minutes \\
\hline 25 & $\begin{array}{l}5-7 \text { minutes } \\
\text { Building to } 55-60 \% \text { HRR }\end{array}$ & $\begin{array}{l}45 \text { minutes } \\
20 \text { min @ 65-70\% } \\
10 \min @ 70-75 \% \\
5 \text { min @ 65-70\% } \\
10 \min @ 70-75 \%\end{array}$ & 5-7 minutes \\
\hline 26 & $\begin{array}{l}5-7 \text { minutes } \\
\text { Building to } 55-60 \% \text { HRR }\end{array}$ & $\begin{array}{l}45 \text { minutes } \\
20 \text { min @ 65-70\% } \\
10 \text { min @ 70-75\% } \\
5 \text { min @ 65-70\% } \\
10 \min @ 70-75 \%\end{array}$ & 5-7 minutes \\
\hline 27 & $\begin{array}{l}5-7 \text { minutes } \\
\text { Building to } 55-60 \% \text { HRR }\end{array}$ & $\begin{array}{l}50 \text { minutes } \\
20 \text { min @ 65-70\% } \\
10 \text { min @ 70-75\% } \\
10 \text { min @ 65-70\% } \\
10 \text { min @ } 70-75 \%\end{array}$ & 5-7 minutes \\
\hline 28 & $\begin{array}{l}5-7 \text { minutes } \\
\text { Building to } 55-60 \% \text { HRR }\end{array}$ & $\begin{array}{l}50 \text { minutes } \\
20 \text { min @ 65-70\% } \\
30 \text { min @ 70-75\% }\end{array}$ & 5-7 minutes \\
\hline 29 & $\begin{array}{l}5-7 \text { minutes } \\
\text { Building to } 55-60 \% \text { HRR }\end{array}$ & $\begin{array}{l}50 \text { minutes } \\
20 \text { min @ 65-70\% } \\
30 \mathrm{~min} @ 70-75 \%\end{array}$ & 5-7 minutes \\
\hline 30 & $\begin{array}{l}5-7 \text { minutes } \\
\text { Building to } 55-60 \% \text { HRR }\end{array}$ & $\begin{array}{l}50 \text { minutes } \\
20 \text { min @ 65-70\% } \\
30 \min @ 70-75 \%\end{array}$ & 5-7 minutes \\
\hline
\end{tabular}


Appendix E: Anthropometric Measurements

Participant \#:

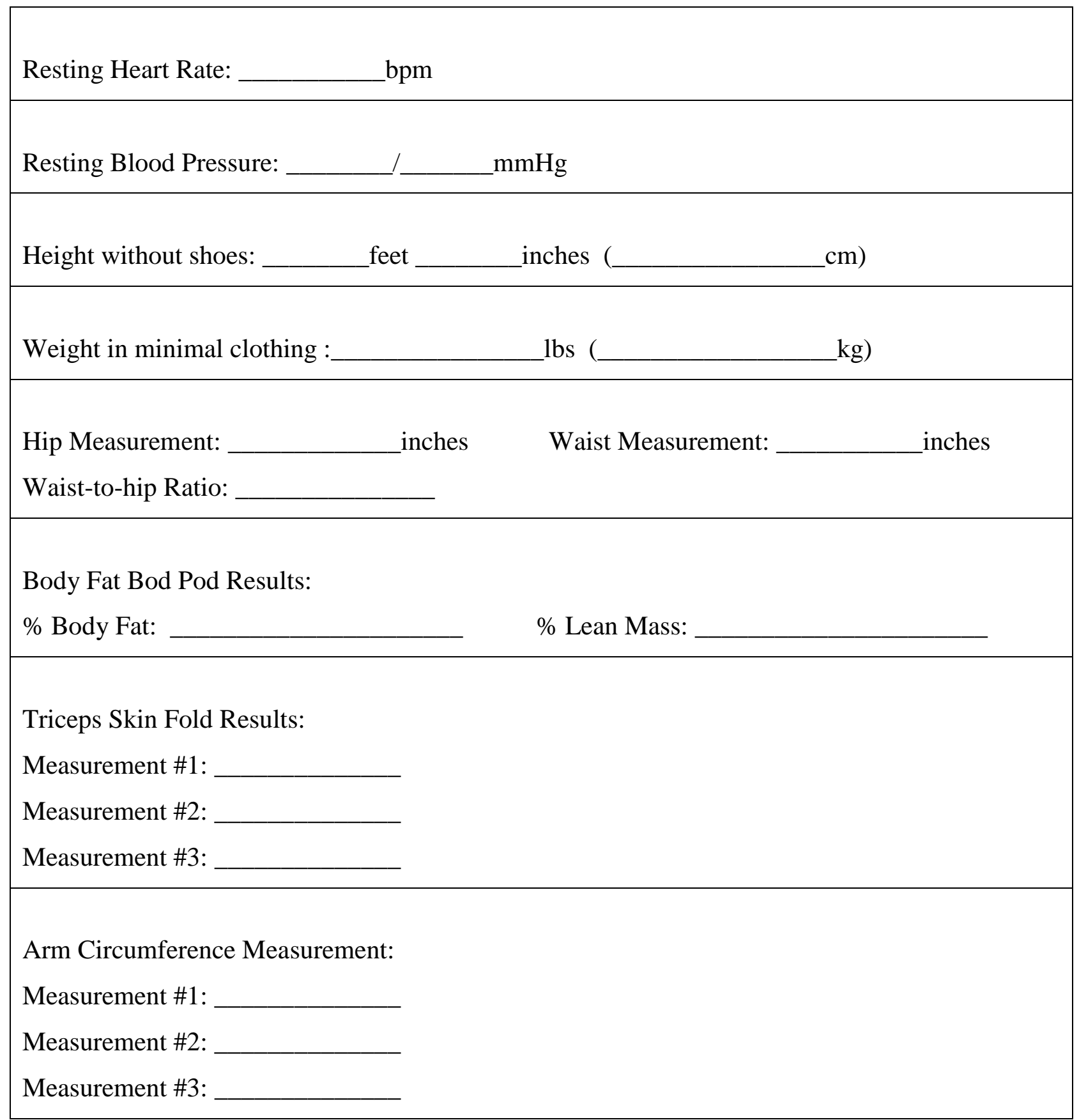


Appendix F: Psychosocial Mediator Surveys

Participant Number:

PRE / MID / POST

\section{Survey \#1: Barriers to Exercise}

For each item, indicate how often it interferes with or prevents you from exercising. The response options are:

$$
\begin{aligned}
& 1=\text { never } \\
& 2=\text { rarely } \\
& 3=\text { sometimes } \\
& 4=\text { often } \\
& 5=\text { very often }
\end{aligned}
$$

How often does any of the following interfere with or prevent you from exercising?

1. not having someone to exercise with

2. having other people discourage you

3. feeling self-conscious

4. being afraid of injury

5. not having time

6. feeling too tired

7. not having a safe place to exercise

8. being in pain

9. being afraid that exercise will cause pain

10. not having a place close to you to exercise

11. not having transportation to get to an exercise facility

12. having to take care of someone else

13. not being able to afford exercise membership fees 


\section{Survey \#2: Importance of Motivators to Exercise}

Please rate the following by their level of importance to you. $1=$ not at all important; $6=$ very important

$\begin{array}{llllll}1 & 2 & 3 & 4 & 5 & 6\end{array}$

not at all important $\rightarrow \rightarrow \rightarrow \rightarrow$ very important

What motivates you to exercise?

Preventing or reducing pain

Preventing or reducing illness

Having a safe place to exercise

Having a place near you to exercise

Advice from your doctor

Information about the health benefits of exercise

\section{Survey \#4: Self Efficacy \& Social Support}

1. How much could you count on those close to you for support and help if you wanted to become more physically active?

not at all, very little, somewhat, A lot

2. How sure or confident are you that you can start or continue to exercise for 30 minutes per day at least 5 days each week (or a total of 150 minutes)? Very unsure, somewhat unsure, somewhat sure, very sure

3. How has your non-aquatic class exercise changed during the time you have been participating in this program?

Decreased, Stayed the Same, Increased 
Please circle the number that represents how confident you are that you could participate in water exercise in each of the following situations.

$\begin{array}{ccccc}1 & 2 & 3 & 4 & 5 \\ \text { Not at all } & & & & \begin{array}{c}\text { Extremely } \\ \text { Confident }\end{array} \\ \text { Confident } & & & & \end{array}$

1. When I am tired

$\begin{array}{lllll}1 & 2 & 3 & 4 & 5\end{array}$

2. When I am in a bad mood

$\begin{array}{lllll}1 & 2 & 3 & 4 & 5\end{array}$

3. When I feel I don't have time

$\begin{array}{lllll}1 & 2 & 3 & 4 & 5\end{array}$

4. When I am on vacation

$\begin{array}{lllll}1 & 2 & 3 & 4 & 5\end{array}$

5. When it is raining or snowing

$\begin{array}{lllll}1 & 2 & 3 & 4 & 5\end{array}$

6. When I am sore

$\begin{array}{lllll}1 & 2 & 3 & 4 & 5\end{array}$

7. When I fear the exercise will cause pain

$\begin{array}{lllll}1 & 2 & 3 & 4 & 5\end{array}$

8. When I have to exercise alone

$\begin{array}{lllll}1 & 2 & 3 & 4 & 5\end{array}$

9. It is difficult to get to the exercise location

$\begin{array}{lllll}1 & 2 & 3 & 4 & 5\end{array}$

10. I felt self-conscious about my appearance $\quad \begin{array}{llllll}1 & 2 & 3 & 4 & 5\end{array}$ 


\section{Appendix G: 6-Minute Walk Protocol}

\section{6-Minute Walk Test Protocol}

Participant \#:

Date \& Time of Test:

PRE / POST

Have participant sit and rest near the start line for at least 10 minutes prior to the walk test begins. There is $\underline{\mathrm{NO}}$ Warm-up prior to beginning this test.

Take Resting HR:

bpm

Take Resting BP: $\mathrm{mmHg}$

Ask the participant: "Did you take any medications today? If so, could you tell me what you took and what time you took it?

Instruct the participant:

"The object of this test is to walk as far as possible in 6 minutes. You will walk back and forth in this hallway at your own pace. You are permitted to slow down, to stop, and to rest as necessary. You may lean against the wall while resting, but resume walking as soon as you are able. If you experience chest pain, shortness of breath, leg cramps, or extreme discomfort, please stop the test and let us know about your symptoms.

You will be walking back and forth around the cones. You should pivot briskly around the cones and continue back the other way without hesitation. Now I am going to show you."

\section{TESTER: DEMONSTRATE HOW TO TURN AROUND THE CONES}

"I am going to keep track of your laps on this worksheet. I will count a lap every time you turn around at this starting line. Remember that the object is to walk AS FAR AS POSSIBLE in 6 minutes, but don't run or jog.

Are you ready to begin? When I say 'GO' please start walking."

Have participant stand at the start line, instruct participant to 'GO' \& begin timer.

**Do NOT talk to participants during test except for below script. Use an even-tone when delivering scripted statements.

** If a participant stops walking during the test and needs to rest, tell them "you can lean against the wall if you would like; then continue walking whenever you feel able." Do NOT stop the 
timer. If they refuse to continue or feel that they cannot continue, offer them a chair to sit and rest. Note the distance, time that they stopped walking, and reason given.

At the end of the first minute tell the participant:

"You are doing well. You have 5 minutes to go."

At 4 minutes remaining, tell the participant:

"Keep up the good work. You have 4 minutes to go."

Record RPE

At 3 minutes remaining, tell the participant:

"You are doing well. You are half-way done."

At 2 minutes remaining, tell the participant:

"Keep up the good work. You have only 2 minutes left."

Record RPE

At 1 minute remaining, tell the participant:

"You are doing well. You have only 1 minute to go."

At 15 seconds, say:

"In a moment I'm going to tell you to stop. When I do, just stop right where you are and I will come to you."

At the 6 minute mark, say: "STOP!" walk over to the participant and mark their placement in the hallway with tape.

Take HR: bpm

Recovery Heart Rates:

Take BP: $\mathrm{mmHg}$

Record HR at 2 min post

Record Borg RPE:

Ask participant: "What, if anything, kept you from walking farther?"

Record Total Number of Laps: Record Additional Distance covered:

Calculate the total distance walked, rounding to the nearest meter Number of laps (x 200 meters $)+\frac{}{\text { additional meters }}=\frac{}{\text { Total Meters Covered }}$

Did the participant stop or pause during the test? YES

NO

Reason:

Calculate VO2:

$\mathrm{VO}_{2}=20.05+(0.019 \times$ Distance walked in meters $)-(0.278 \times \%$ body fat $)$

$20.05+(0.019 x$ m) $-(0.278 \mathrm{x}$

Distance \% Body Fat $\mathrm{VO}_{2}$ 


\section{Appendix H: Focus Group Guide \\ Focus Group Guide}

\section{Introduction to be read to the focus group prior to beginning the inquiry:}

I would like to thank you all for lending your time and thoughts. My name is Jim Rye and I will be moderating the group, and this is Sam Zizzi who will be acting as the recorder for our session today. Let's take a moment to go around the room and introduce ourselves. If you could let everyone know what your first name is and why you chose to participate in this study it will help us to get to know one another a little better.

During this focus group session, I will be asking questions in hopes to get all of your individual input. All of the information and ideas that you offer during this time is important to me so I would urge you to share your thoughts, experiences and ideas freely. All comments will remain confidential. Feel free to speak up anytime, but please speak one at a time and allow others to finish their comments before interjecting. You are free to address one another or the moderator; I would like to keep this casual and conversational. All comments are welcomed, both positive and negative. I would really love to get your truthful feelings concerning the discussion topics.

This session will go on for no longer than 1 hour, with no break. Feel free to help yourself to the provided refreshments and if you need to take a restroom break they are located (state location).

The primary purpose of this meeting is to see how you feel about the aquatic exercise sessions, any barriers you encountered with participation, and the effects it had on your lifestyle now that the study is over. I would also like to know how you relate exercise to your cognitive abilities as well as your candid thoughts on the project as a whole. Please keep in mind that there are no 'right' answers. All comments are welcome and will help give insight to allow the recorder and me to make adjustments and improvements to future study design. All comments will remain confidential. I am also asking you please to not share by name outside of this group something that another participant says (e.g., "John Doe said he has no social support from home.”) 


\section{Information goals for the Post-Program Focus Group:}

1. Find out how the participants feel about exercise in general and specifically water exercise and if these views have changed from their initial thoughts (related to exercise self-efficacy).

2. Identify if the participants exercise barriers have changed. (assisting in identifying the new/current barriers \& strategies they have adopted to deal with these barriers)

3. Do the participants relate their ability to perform ADLs (activities of daily living) to exercise (ADL self-efficacy related to exercise/ exercise importance)? Has this changed from the pre \& mid-intervention thoughts? Do they attribute any positive/negative changes in ADLs to participating in this project?

4. Do the participants feel they will continue to participate in regular exercise now that the intervention is over? Are they actively seeking out a new means of physical activity? Do they have a plan for continuing physical activity? (learned behavior \& exercise importance)

5. Have the participants increased their physical activity levels outside of the intervention?

6. Have their social support systems changed? Do they view the exercise group as a social support system (social support)?

7. What type of exercise environment do the participants prefer? Does this alter their willingness to participate in exercise? (self-efficacy \& adult learning related to environment)

8. Have the participants views on relation between exercise participation and cognitive function changed? (exercise importance) 


\section{POST-EXERCISE INTERVENTION QUESTIONS:}

-Now that you have completed the program, how do you feel about exercise?

-What did you like best about the aquatic exercise classes?

-What did you like least about aquatic exercise classes?

-Do you think that you will continue to exercise now that the program is over?

-If so, what type of exercise do you think you will participate in?

-What type of supports do you think would help you to continue exercising?

-How do you think exercise affected your cognitive function: thinking processes, remembering, learning?

-Do you have any personal examples since you have started this program?

-In what ways does exercise affect your daily activities?

-How do you think your daily activities have changed since you have been exercising?

-What motivated you come to the aquatic exercise classes?

-What discourages you from coming to the aquatic exercise classes?

-What were the barriers you encountered during this program?

-What solutions and strategies did you come up with to deal with those barriers?

-Did your barriers change throughout the program or did they stay the same? If they

changed, why do you think that happened?

-Who supports your decision to participate in exercise?

-Tell me how they have supported you while you have been participating in this program. 


\section{Appendix I: Focus Group Transcripts}

Focus Group A

Intro:

Jim: This little focus group we are doing today is, is, you know we have to run it through our human compliance, our human research compliance group. And so they give us some guidelines, some terms, of what we can and cannot do, so I do pretty much have to read this whole focus group guide script to you ahead of time. So just sort of bear with me, and if you have any questions at all, just stop me and then we will get to the questions. Okay. So. Thank you for lending your time to give me your thoughts and ideas. I'm Jim and I'll be moderating the group, and this is Pete who will be acting as the recorder for our sessions today. So let's take a moment to go around the room and introduce ourselves. And, um, I can see everyone's names, but what I would like to do is if we could just briefly just tell us a reason why you chose to participate. Anything you'd like to say. Or why don't we go clockwise so we'll start with Ann.

Ann: Okay. I'm Ann. I have been doing aerobic, or exercise in the pool at the water park, so this was a chance for me to learn some new exercises and what to do and have some professional somebody teaching me. And I thought, well it would be a good idea, it was free. The water exercise was free. But it has been very interesting.

Jim: Thank you. Jane?

Jane: I'm Jane. I've been exercising off and on I guess since the late 80s, and I was exposed to water exercise this summer through Bopark. And I enjoyed it and thought when I saw this in the paper I called about it, and I was accepted. It's a different type of exercise and it keeps me moving.

Jim: So you had some prior exposure to it?

Jane: That, that was the first time I had ever been in the water. And I'm no swimmer, and I definitely have a fear of water. So some of the things, when you take both feet off the floor (laughs), you lose me. I'm not good at that.

Jim: Okay. Tina.

Tina: I'm Tina. And I have a lot of arthritis, so I find it difficult to try on land. And I had never done aerobic exercise, but I'm sold and bought. So, I love it, and I see the difference I can do things I couldn't do.

Jim: Wow. Thanks. Okay, Bob?

Bob: I'm Bob. I got into it because my wife wanted to. Otherwise I wouldn't have done it, but on the other hand, I'm glad I did. I've lost weight, feel good about myself. 
Jim: so your wife encouraged you to?

Bob: Okay, that's my wife.

Jim: So is that why it says "P" afterward, for persuader? * group laughs* Meg?

Meg: I'm Meg. I have been wanting to exercise and wasn't doing it regularly, and by signing up for this it was a way to make me do it 3 times a week. And I had had a class with Lori a couple years ago in the deep end and I knew what a good teacher she was so that encouraged me too.

Jim: Wow. Nice compliment. Jill?

Jill: Previously I have done water exercise and I found it quite fruitful and helpful for me. And I do have arthritis starting and I've found that I'm able, since I've been coming to this, I've had more movement. It's been easier for me to get up and, um, it's been a great class. She just encouraged and pushed us to the place where it makes you want to do more. So I've given an A+.

Jim: Wow, these compliments. So, some of you are speaking of some things that we will have questions on, so feel free to bring them up again to the extent that you'd like to. Pam?

Pam: I'm Pam. I used to walk quite a bit with my dog, and she got older and I got older and retired, and I've got extensive arthritis so I just quite doing anything, which obviously didn't make me feel very good. I hurt my knee badly 2 years ago and I wasn't able to do anything and I thought maybe in the water I could move it. So I saw this advertised and thought I'll try it. And I don't like pools, and I don't swim, and I don't like water (laughs), but I thought I'd try it. And it made my knee better. I don't have to where my brace to do stairs, so I'm very pleased that I tried it.

Jim: Great. Well, thank you all. I'm just going to sort of scoot through the rest of this, and then we can get to the questions. So, during this focus group session, I will be asking questions in hopes to get all of your individual input. All of the information and ideas that you offer during this time is important to me so I would urge you to share your thoughts, experiences, and ideas freely. All comments will remain confidential. Feel free to speak up anytime, but please speak one at a time and allow others to finish their comments before interjecting. You are free to address one another or the moderator. I would like to keep this casual and conversational. All comments are welcomed, both positive and negative. I would really love to get your truthful feelings concerning the discussion topics. This session will go on for no longer than 1 hour with no break. Feel free to help yourself to the provided refreshments and if you need to take a restroom break, they are right outside here. I think there is a little poster beside it. So the primary focus of the meeting today to see how you feel about the aquatic exercise sessions, any barriers you encountered with participation, and the effects it had on your lifestyle now that the study is over. I would also like to know how you relate exercise to your cognitive abilities as 
well as your candid thoughts on the project as a while. Please keep in mind that there are no "right" answers. All comments are welcome and will help give insight to allow the recorder and me to make adjustments and improvements to future study design. All comments will remain confidential. I am also asking you to please not to share by name outside of this group something that another participant said. An example of this is "John Doe said he has no social support from home."

\section{Question 1:}

Jim: Okay, any questions before we start? Alright. What worked well with the last group was I just sort of held it up like this. So, okay. So now that you have completed the program, how do you feel about exercise?

Ann: it's beneficial, I know that, but at my age, if I exercise too much, I will end up in a chair and take a little nap afterwards, but I've always known exercise was beneficial. I've just kind of ignored it for a long time, and not really do what I need to do.

Jim: so you've been aware that exercise has been beneficial?

Ann: Well, when I first moved to Morgantown, they had a Y. Well, I went down there for exercise just to meet people and also to exercise. I didn't lose weight, but I lost inches just from that. I didn't diet, but I did lose inches. And it made me feel an awful lot better.

Jim: Thank you Ann. Anybody else have any thought about it?

Jane: It is important because all of us as aging and kind of forget that you can't do a whole lot of things if you don't have the real resolve to keep your house clean and etc. and so forth. It does help you to do your activities and everything at one time. So, you learn it does help. There is no doubt about it. If you don't use it you lose it.

Jill: That is exactly right. I mean I think it keeps you in shape, not only physically but even mentally.

Jim: Anybody else?

Meg: I think it does give you more energy throughout the day and it helps you move better an awful lot too.

Ann: it improves your mood too. (group agrees)

Question 2

Jim: I apologize; I thought I had these in order before we started. I thought I held up a different question than what I asked. You were all responding to how you felt now that the program is 
over. So, now if while we are doing this you have something to say in response to something we asked in the past, feel free to do so. Okay, now that I think we have the right order. So, do you think that you will continue to exercise now that the program is over?

Jill: yes, I think so.

Tina: yes, I know so. We're going to join the other aerobic class. The paid part.

Jim: So you are going to pay for it?

Tina: Right. Yeah. We are going to continue. It's very easy and there is a difference. First month after I leave here, I always go and do some more stuff at a chiropractic place, and get a massage, then go home. For the first month, I was so dead tired by the time I got home at night that you, just went to sleep, you would just sit down on the chair and you were done. And I don't even nap anymore now. The energy is so much better.

Jim: So you're (can't understand what is said). Anybody else have any thoughts or response they would like to give to do you think you will continue to exercise now that the program is over?

Jill: um, at the senior center there is a class two days a week up in the Wiles Hill area. And I probably, its 2 days, anyway, I will probably continue that either next month or the first of the year for sure. And perhaps get into some more water because I find that it is beneficial to me.

Jim: well, thank you Jill. Anybody else have anything to say for this one?

Ann: I'm going to book a hotel, down there that has a pool and a weight room. So I figured that with my new knowledge of what exercise is from the pool that'll be more beneficial and then I'll have access to other things too.

Jim: Great. Thanks.

Jane: I think I'll do dry, dry land (laughs). And wait until, I'll see since I've never compared it and see how that goes. I'll probably do the same thing that Jill is going to do because I had done it before, so we'll see how it works out.

Pam: I'm kind of afraid that I won't continue to exercise. This was a little overwhelming for me 3 times a week in the middle of the day. I'm not a morning person, but like a 10 or a 10:30 I think I could have handled, and then I wouldn't have met in the middle of the day kind of takes my whole day. I babysit and I volunteer some, but I am maybe hoping to find something like Jill was saying maybe a couple times a week, or something that would be flexible. Like the HealthSouth arthritis pool might be good so I've heard for me, so I'll probably look into that. Cause I do appreciate walking without pain.

Ann: HealthSouth is very beneficial. When I first started I was having some problems with my balance, I asked my doctor if he thought water might help, and he said "well, it's worth a try." So 
I went out to HealthSouth and I don't know if I did the whole course, but I live on the mountaineer mall side of town and the hotel is closer to me. So I got wind of this and that's why I changed, but I had access to exercises at HealthSouth...not HealthSouth, HealthWorks... with the group.

Question 2a

Jim: So for some of these questions, we have some sub-follow up questions. You have already provided some response in relation to some of these questions. Again, if you feel you want to, you can bring it up again. So the follow up question here is: If you plan to continue, what type of exercise do you think you will participate in?

Tina: Water. Both of us.

Meg: Yeah, I'm going to sign up. My only disappointment this whole study was my husband got in the control group. He is going to be enrolled next semester, so I'm going to sign up too. We'll do it together then.

Jim: Great. Anybody have anything else to say about what types of exercise you think you will participate in?

Ann: I may be forced to take up walking. My husband walks 20 miles a week, and he has been preaching to me that I need to walk on a regular basis. And I don't like to walk in the summer outside and I don't like to walk in the cold in the winter. That's why I'm going to join the hotel. But I haven't lost any weight, and then he is very disappointed in that. He doesn't realize that I'm still able to get around, but he wants me to lose weight. So I may have to start walking. I'll go Monday, Wednesday, and Friday to swim, and walk on Tuesdays and Thursdays. I may have to do more than I'm doing to lose weight.

Tina: There is a treadmill. You could always do the treadmill.

Ann: yeah, I can do that down there too. I'll take a few days to swim and also a few days to rest.

Jane: Yeah, you're supposed to relax your muscles in between a day or something.

\section{Question 2b}

Jim: So, um, what you said was a nice lead in to the next question. So, what types of supports do you think would help you continue to exercise?

Ann: You've heard my story. (laughs)

Tina: I think most people need a buddy system. Whether it is someone at home or prior to my husband retiring, my daughter and I always exercised and did everything together. I really think you need that support from somebody going with you or being there. And, you know, if you 
wake up and it's a lazy day, you've got somebody there telling you to get your stuff on cause we got to go.

Jill: I think it's a commitment. You set the time, and then you do it because you made that commitment. In other words, if you are going to meet someone to walk or if you have a set time like at the senior center, although it's early. I think it starts at 9. And you know you have to be there around quarter till. So you know, once you make that commitment, you follow through even though it is hard some mornings.

Ann: if you make an official commitment. It's one in the main things.

Tina: Life gets in the way sometimes. You'll think, oh I got to go do something or I've got to go exercise and then the phone rings, and you answer or before you know it or you stop here or there, the day is over. Like with the diet plan, I've lost 16 days this month on the diet plan.

Jane: And if it's a cold wet foggy morning, covers feel much better in your bed than getting out, but I know I've got to get out. (laughs)

Jim: Well, how about this one? So, how do you think exercise affected your cognitive function: like your thinking processes, remembering, learning?

Jane: It has to do something to you whether you realize it or not. It has to. It has to affect the whole body, your whole being.

Questions 3

Jim: well, um, just a reminder. You aren't going to hurt anyone's feelings by being as frank as you feel you should be. You aren't going to hurt anybody's feelings. Please be frank! Any other response to how you think exercise affected your cognitive function?

Pam: Well, I guess I was struggling before and I was hoping it would help me. I stumble for words sometimes. I certainly stumble remembering things, and I'm afraid that some of the things I've done have been worse. And I struggled very much with the different steps. I was okay with something regular, but if we had to move four parts of our body different ways at the same time, I struggled with that. I can't focus on one part and try to get the other part. But I'm interested to see when we take our tests to see if I've improved at all. I know I've made even bigger mistakes while driving. You know, I got out of the car at the grocery store with the car running. See, I really am struggling. But my husband says you may not have improved, but it could have been worse. You know, I know exercise endorsed me very good emotionally. I was very depressed when I started and I'm much better now. So I know there is a lot of benefits to it, but I feel like it's important to keep trying even if you aren't getting the results you think you should have gotten. I don't want to mess up her results with me (laughs), but that's how I feel. 
Jim: well, you know, the process for us to do our research involves the truths value. So please be as frank as possible.

Ann: I'm anxious to see what my, you know, one or two of the things I thought was simple. And when we got to 4,5,6 then I got all messed up and all, but I'm anxious to know. My husband, he suffers from dementia some, and I think the reason he pushed me into this was because if I got it too, then that would be the end. Between the two of us, it's one of us (laughs). He makes up for what I can't and I make up for what he can't do.

Jill: Um, my coordination isn't the best in the world, and I find by exercise or repetition like we did in the class, that I saw a big difference at the end of the class than in the beginning. Maybe you know when we did some exercise on one leg or you know, I didn't always do it just right, the way they were doing it on land (laughs), but you know, in the end, I felt I was much better in following.

Question 3a

Jim: Some of you have cited some examples, that the follow up question to this is: Do you have any personal experiences since you started the program, you know, ways in which exercise has affected you? Anybody want to add anything?

Tina: I can touch my feet now. I can bend over and touch my feet. I couldn't before. We like to place cards occasionally, and when we drop cards, I could barely reach down to get them. But now I can pick cards up. I noticed a difference on the stairs for me. I can actually get to the top and don't have to stop and can't breathe now. There is a difference. Still got a long way to go, but there is a difference.

Ann: I think my balance has increased. There for a while, especially on ground, it was rough. I leaned to the left, and now, I'm not leaning to the left as much as I used to.

Question 4

Jim: well, I guess some of your responses have lead nicely into the next question. And that is in what ways does exercise affect your daily activities?

Meg: I think my balanced has gotten a lot better too. Because I always try to, I've always heard that, I don't know it its true or not, but if you practice putting your socks on by just standing on one foot, that it will help. I never could do it, but I can now. So I think it's helped my balance a lot and my knees are better too.

Ann: My knees feel better too. Definitely better too. I don't think my knees have been bothering me as much. 
Tina: I have muscles for the first time in my life. I've got leg muscles and arm muscles. I mean, they were always there, but they were like nonexistent. I could barely use them. I have muscle now. And I have to be better.

Pam: I've increased my daily activities doing this because like you said, a commitment to have to be here and move that, and it moves a lot of stuff of what you have to get done at home and where you're going, and prioritize. And I made this a priority. I signed up and I wanted to stick with it, and I loved the people. That was great, but it certainly got me off the couch.

Ann: If you aren't using your brace, then that's a big plus right there.

Pam: now I can take stairs one step at a time, like these stairs we came up today. I can do that, but I couldn't at the beginning. It's scary because I'm afraid I'll lose it.

Ann: well, going from depending on a brace to not having to, that's got to be a big plus.

Jill: Well, we went from raking leaving to shoveling snow, so I had to do a little of both. I found it a little easier to bend and move from this class.

\section{Question 4a}

Jim: So again, so the answers you've been giving are related to this follow up question. And it is: How do you think your daily activities have changed since you have been exercising?

Pam: I answered that. Mine has increased a lot.

Bob: My activities haven't changed, but I don't have as much lower back pain as I did before I started.

Tina: You can move your shoulder now.

Bob: Yeah.

Tina: You couldn't put your arm up like this very well.

Jill: In other words, she wasn't getting enough hugs. (laughs)

\section{Question 5}

Jim: Thank you. We'll move onto a question now about motivation. What motivated you to come to the aquatic exercise classes?

Bob: I answered that one already. (laughs)

Tina: I have a friend in the morning class. When she got wind of it, she let me know. And you know, heard it through the grapevine. 
Jim: So that motivated you to come to the class?

Tina: well, we actually got lucky. I'm actually not 60 yet, but she made an exception because he was over 60 . So she made the exception because of that and because I really needed it.

Ann: I'm just wondering, what age did they ask you? I'm going to be 80 in February. I'm just wondering what the actual range was.

Jim: I haven't had anyone in the focus group tell me they were 90. (laughs)

Ann: I am proud of being $80 \&$ being able to do what I can do being $80 \ldots$ I know a lot of people that are younger than I am that can't.

Jim: I think the age gap was 60-90.

Ann: I was just wondering what the average actually turned out to be. It's none of my business, but I was wondering.

Jane: I think she is right in the fact that the older. I really feel the older you get, the more proud you should be. And if you are up and about, you should be proud of your age. Unfortunately we are a youth oriented country. Everybody, all of we gray hairs are not too popular anymore. Ann's going to be 80. I was 78 in July. I feel that that's you know...

Jim: Congratulations

Jill: I was 75 .

Question 5a

Jim: Well, how about some things you liked best about the aquatic exercise? What was the things you liked best?

Tina: The water.

Ann: Well, it could have been warmer, but. *group laughs*

Tina: I don't think we would have exercised as well if it had been warm water. We had to exercise to keep warm.

Jill: we did a lot of sharing. Personal lives, that was wonderful. Encourage, I think we encouraged one another. It was just good. We had a lot of laughter. It was exercise but you really enjoyed it.

Jim: So it sounds like you really enjoyed it?

Jill: Oh yeah. 
Ann: while we were in the water, I didn't say it, but I can get my foot up under my chin in the water, but on land I can't even get it up to my waist. So just being able to use my body in an easier way a plus for me because if I get down on the floor, it is really a chore to get back up.

Jim: so that's something that you liked that you were more mobile in the water?

Ann: Yes, I'm more mobile.

Jim: I saw some heads shaking in agreement with that statement. And I also saw some heads nodding in agreement with the coldness of the water.

Jill: Oh yes. And I think that even with the instructor, it was, she made it fun. And..

Tina: They all did. They were a wonderful group of people.

Jill: Yeah, they young people. They were superb.

Ann: I didn't even feel like I was exercising. Really. I mean, I knew that I was putting a stress on, and when we were trying to go real fast I was getting short of breath. I knew I was exercising then, but just with I (?), getting my feet and legs and hands and all going in the right direction, it's a challenge for me. But I kept going, and I can't say that I did the exercises right, but I kept moving. And it was a very good experience.

Meg: I liked the stretches too. I thought that was really a good part of it.

Tina: Almost a little bit like yoga.

Meg: Yeah. A little bit. I mean, it was just really well down. And the fact that you stretch to one side and then across, I think it was just really well done.

Jim: Thank you Meg.

Question 6

Jim: okay, now let's go to the other side. Again, please be frank. We understand everyone's true perspective here so we can make the programs better. What discouraged you from coming to the exercise class, the aquatic exercise classes?

Jill: The cold water. *group laughs*

Pam: It was hard to park sometimes. That was a trick to learn. To come early enough to find a parking place or gage that.

Tina: I think parking was the worst.

Pam: the water, I mean, it chilled you at first, but once you got moving, it was okay. 
Jill: It was okay.

Ann: It was more a big joke.

Jill: Yeah, it was more of a big joke.

Ann: It wasn't too bad.

Pam: But I think with me too, I lost whether.. I'm probably the one that struggled with the coordination and trying to do the steps they wanted me to do, plus keep my heart rate up. If I couldn't do the steps, then I didn't know whether I should just keep moving to keep the heart rate up. I was thinking in struggling to do the steps, I was helping my brain make different paths. So I struggled with that part. I was unsure whether I was supposed to just keep moving or, I just kept trying to do the steps. But I'd laugh at myself and fall over a lot (laughs), which is hard to do in the water.

Jill: I laughed at you too going around and around.

Pam: Well, see, I made everybody smile.

Jim: That is one of the things that I understand you were having problems with was trying to be coordinated. Now I don't want to put words in your mouth. I just want to understand.

Pam: And that changed from when I started I really struggled and I was almost more depressed because I couldn't do it right, and I thought how is this going to help me. I'm not doing it right. And my daughter and my husband were very good. My friends, you know, just keep going. Just moving, keep doing it. And I noticed today, everything felt so good in the water. And you knew the steps and there is still stuff I struggled with, but it was a whole different mindset of how it was going.

Ann: You stressed yourself trying to be perfect when it wasn't a perfect...

Pam: When I'm not*laughs*

Ann: You weren't supposed to be perfect.

Pam: well I didn't want to be perfect. I just wanted to do it right to get the benefit, and I there was times that I just couldn't.

Ann: You just put too much stress on yourself. Not that they forced you.

Pam: True. True.

Jim: well, nevertheless you're saying in the big picture, it wasn't discouragement. 
Pam: We had to accept that I wanted to come see the people and come. I do. You all were a big draw for me. That's why I think the buddy system is such a good idea, just to have a friend and make it a priority and just know that it is helping. You just make up your mind that this has got to be helping.

Jill: You had bronchitis also, and so, but I think that, in my personal opinion, I think that this exercise actually helped you recover faster. I do. It makes you try to breathe.

Tina: It takes the breathing from your lungs and makes you breathe harder. Never thought about it that way, but it is true.

Pam: well, I couldn't come a couple times it was so bad, but when I did come, I didn't want to get anybody sick. But I did think that moving was better than not moving. Plus if you lay down, you just cough all the time.

Ann: I had a cough when I came and I thought, well I'll probably scare everybody off coughing all over the place. And I got over in the corner as far as I could get and everything, but it was just something that it had to take time to get rid of the cough.

Meg: I had some health problems that discouraged me too. I had a dental things going on, and that's where I missed a couple classes. And then there is always traffic, because I live on the other side of town, and one day I sat in traffic for 30 minutes trying to get. I mean, and then, I was like there's no point in going 30 minutes late. Morgantown traffic is just terrible. *group agrees*

Tina: We drove. We live half an hour from here, but we don't really hit any traffic. We just get off the interstate. We live over in PA, so going to the coliseum works really well for us. You only have the boulevard and here you are.

Jane: yeah, well you all come from across town. That's commitment.

Question 6a

Jim: well, you've given some responses to this already. You've given some examples. So looking at what you like least about the aquatic exercise classes. Is there something you liked least? And maybe you could look at it this way. I liked everything, but there was one thing that maybe stuck out that didn't sit so well with me.

Jane: well, the one thing I said at the beginning that I didn't like was when I had to get both my feet up off the floor. I didn't like, I don't like it. But I tried to do it as best I could. And I'm just not comfortable enough in the water to let myself do it and go under. That's my fear! I mean, I'm afraid to go underneath it. To get my head under. And I didn't do that, but I just, that's the only thing I had a problem with. But I kept trying. 
Jill: I couldn't. I'm the same way because I can't swim. So when my feet leave the ground, I have this fear that I just kept exercising. I did something, whether it was right or wrong. *Jane agrees*

Jim: I think both Jane and Jill shared a good thing, that they both were unable to swim.

Jane: You can't swim either, can you?

Pam: I can side stroke, but I don't do good. I mean I don't have a fear. I can side stroke pretty long in deep deep water. I don't have a fear, but I run out of breath and sink. But I didn't so.

Jill: Just the certain exercise that I had problems with, but the rest I wasn't afraid of the water, you know. And I enjoyed it. It was just when she would say, you know, feet off the ground.

Ann: I don't have a problem. I might the rare one. I have enough fat that I can't keep my feet on the ground. (laughs)

Tina: I had that trouble the whole time. Don't worry. You're worried about your heels pounding. Mine never hit the ground.

Ann: When you were talking about the stretch, I couldn't stretch because I couldn't keep my feet on the ground.

Tina: I had that same trouble. I thought it was just me.

Ann: so, I learned to swim with water wings in the river when I was 4. So I'm not afraid of water. And I will tell everyone that is afraid of water that the fact that you are afraid, and if you could get over your fear, you would have any problems. You've just got to get over the fear. That's why with children, you should teach children to swim when they are young, and then you don't have this problem of being afraid of water. In our generation, most older people did not swim and they then the children got in, and they were so uptight about their children being able to swim that they scared the kids.

\section{Question 7}

Jim: we are learning a lot about ourselves here. So what about barriers? You know, what barriers did you encounter during this program? Were there any barriers that stuck out?

Tina: What do you mean by barriers?

Jim: Barriers are.. Did you encounter any barriers during the program that resulted in I guess you not participating as much as you could have? They could have been barriers in terms of being able to come to the program or they could have been barriers that could have been while you were in the water. Any barriers at all that, if they could have been removed, would have resulted in you participating more fully or freefully. 
Bob: well, we were gone for a while on vacation.

Jim: so vacation was a barrier.

Pam: well, like she said, she had some dental and I had bronchitis. I was ill the last couple weeks and that was certainly a barrier. And like we said before, just the traffic or the parking.

Jane: I was gone on that trip for a week.

Ann: I had to go to Virginia to settle up an estate problem.

Pam: Like Tina said, life happens.

\section{Question 7a}

Jim: What about solutions to any of those barriers that you talked about? Did you come up with any solutions to deal with the barriers?

Bob: I did. I had a noon retirement party to go to, so I came to the morning class. So..

Pam: I did that once too. We were going to travel and I went to the morning class and we left in the afternoon.

Jim: Ah, so you just went to a different class. That's how you removed the barriers.

Tina: I came earlier for a parking place.

Jim: You came earlier for a parking place, yeah.

Tina: Yeah, I don't know where that falls into, but I think they should maybe do something for parking on those game days and things so that, you know, some of them if you stopped and said there was a fellow that said he had a swim class for one hour and I can't walk that far, so they let him go park. And Ann and some of these others that walked from a mile away. And I think they should at least provide since we are only here an hour, they are only there an hour on those days, that they should allow parking for those programs.

Jane: If they ever get this parking at WVU straightened out, the world will probably be ending soon.

\section{Question 7b}

Jim: So, um, while some of you have, why don't we just move onto the next question because I want to make sure I don't keep you any longer. So, um, and again some of you have already responded to this, so if there is something you want to add that's fine. So here's the question. Did, I think I actually have a card for this one. Yes, yes I do have a card. Ok, so, did your 
barriers change throughout the program or did they stay the same? Did they change or stay the same? Any comments?

Ann: well, they didn't change. We just worked around them. That's all I did.

Jim: Don't change, just worked around them.

Ann: Yup, I just worked around them.

Jim: I see a lot of heads nodding.

Pam: I got more confident about doing the harder stuff I think from the beginning to the end. I got better at it.

Jim: So your confidence increased. I see heads nodding for that too. Okay. Oh, did you have something you wanted to share? No. Alright.

\section{Question 8}

Jim: Well, how about, I think their coming back. So, let's talk about briefly supports. This is the last regular question that I have with a little follow up question. So, who supports your decision to participate in exercise? And we are asking in general here. Who supports your decision to participate in exercise?

Tina: My entire family does. My girls, they were just as happy as can be that we were both doing it.

Jill: My daughters in fact, I have one in Michigan, and I talk to her every day. And she'll say "are you going to exercise?" And she said, "oh today is the last day. Now you'll need to continue something." So, and then I have one that lives next to me, and "you're looking better mom." So, family support has been wonderful.

Jim: So your children are the support for you.

Meg: yeah, my husband too. As I go out the door, he will say, "You're looking good. You're looking good."

Jim: Thank you Meg. Does anybody else have anything they would like to add about who supports you?

Pam: My husband and all my friends were very happy I was moving. And they were encouraging along the way. I tried to have them teach me the steps out of the water because they were all coordinated. And they said, just do it at your own speed. Just try to do it at your own speed.

Tina: That was the great thing about the program and Lori and the girls and everybody, but especially Lori. She encouraged you to do the best that you could do, and she accepted whatever 
limitations you had. And she didn't make you feel small. Didn't make you feel funny. Or not like I don't want to go because I'm the dunce in the class. You know. Nothing like that. She accepted us as individuals.

Ann: Her aides were good too.

Jill: I think we as individuals we accepted each other in the same manner, made each other feel comfortable.

Jim: So what I'm hearing you say is not only the instructor, but the instructor's aides, and you as individuals. You all supported each other.

Jill: We were a very happy group. I'm sorry to see it end. *group agrees*

Meg: you know, another thing I enjoyed were her recipes. *group talking and laughing*

Tina: Her runs, her stories. I'd be thinking I'm so tired. I don't think these legs are going to go any further, then she'd talk about riding her bike 150 miles and I'd think "Oh, I can do this." You know.

Ann: If she can ride over 100 miles on Saturday, then I can surely do this.

Jill: They all put the youth back into us.

Jim: So those 100 mile rides were motivators. *group agrees*

\section{Question 8a}

Jim: just a little follow up question, which once again you have already responded to to some extent. So, you've mentioned some supports. So, how have they supported you throughout the program? You've given some examples. Is there anything anybody wants to add about how these different entities and individuals have supported you?

Tina: well, Jesse (one of the students) actually got into the pool and worked out with us a few times. Like 2 or 3 times. And, not that it matters, but it was nice...that you know...

Jim: so one of the instructors got in the pool actually.

Tina: yeah, and participated. So therefore, you felt like they didn't think it was above them. It wasn't just like they were teaching a bunch of women. They got in and actually participated. Checked his heart rate and did the whole thing - gave my husband some competition.

Jim: Anything anybody wants to add about how you were supported by the individuals who gave you support?

Question 9 
Jim: Okay, well, just because we don't want to miss anything. So, we don't want to miss anything that may have come to mind earlier or now, we'll give you a chance to say it. So, are there any thoughts or ideas that you would like to share? Anything at all? Anything you have to say is important.

Bob: I'd like to thank the ladies for making me feel comfortable. *group laughs*

Jane: You were a brave soul. (laughs)

Ann: We're glad you wife was able to be included with being so young. *group laughs*

Tina: Just a few months dear. You know, sometimes people are really into their cut off points, and are real particular. And I admitted to her online through an email. And I didn't lie, but I pleaded with her, really milked it. As long as I go, we've got 6 months, so hopefully she will make that difference.

Pam: I'm glad I did it. I think that's the bottom line is I'm glad I did it.

Tina: I'm glad there is something else going on that we can continue it. You know. I'm not somebody who, I don't mind being in water that is 5 feet. If I can just get by knowing I can touch the ground, then I'm comfortable. But 13 and 9 foot pool really intimidates me. But it's nice to know we can still do the other ones and still do that one until I get my nerve better.

Jim: So you are glad there was an opportunity to continue. It sounds like, I'm not as acquainted as you are, but it sounds like there was some flexibility in terms of what you participate in.

Tina: yeah, we can be with the control group, and she said we can still be in that group. We are just paying a fee now. But saying we don't want to be in that one one time, we can go over into the deeper pool.

Jim: oh that's what you were talking about.

Tina: Yeah. So we have the flexibility to go back and forth and maybe ease us into, you know.

Ann: I knew that it was available, but I just never pursued it to find out what it took to get into it. And if I could get back into the hotel at \$35, then I will. But if not, then I'll probably come back here for $\$ 30$. And then there are some people in I don't know if any of you all, but your insurance will pay for it, for your exercise. I think Humana does. But I don't have anything that says mine does. There are some, and then there are others that, I have a friend. She goes to an aquatic class. I think it's through WVU. I think she goes to a yoga class, but I'm not sure where that's at. There are exercise classes that are part of the university. Just know where to call and find out about them

Jill: I think that we, and I speak for myself as a senior, and um, my insurance doesn't pay for it, but you are on a fixed income, it would be nice for some of the programs to consider giving a 
senior discount. I know that I'm, I am just so thankful that I was able to get into this program and the price was definitely right. But I did think that is something that the university, that the program should think about. Because I think they would have more people to do it.

Jim: so providing some sort of a financial subsidy or..

Jill: Yes.

Ann: I know it has been about 8 or 9 years since I was going to HealthWorks, but it was about $\$ 50$ admission fee, then you paid $\$ 41$ a month. Now it may be more than that now, but that's why I left there and went to the hotel because at that time it was $\$ 30$ and you had to pay for parking. Now it's more But after I was there a while they upped it to $\$ 35$. Now its more than that now but I got grandfathered in so mine still remains \$35 \& I'm hoping - I've been out for 3 months - I hope they let me come back. There are things available but they are all financial

Jim: any final comments??

Meg: They should just keep up the good work!

\section{$\underline{\text { Focus Group B }}$}

Jim: Let's briefly go around and tell us your first name and how come you wanted to participate in the study.

Pam: Pam...I saw the article in the paper and thought it was interesting but didn't think a whole lot about it and I have a cousin who needed to exercise and had mentioned that her doctor recommended aquatic therapy so I suggested if she would sign up I would sign up to encourage her. So I did and she's in the control group and I'm in the exercise group (LAUGHS), but I have found it to be a very pleasant experience \& I'm blessed that I've been doin it.

Patty: I saw it in the paper and I was more or less curious to what water aerobics was, and I've enjoyed it and I'm glad and I would do it again. And I'm the oldest in the group. I've met a lot of nice people and I've enjoyed the young girls.

Betty: Betty...I saw the advertisement at Kroger and called the phone number from there and now I'm here. I just think every extra exercise, or whatever you do, is good.

Susan: I have a granddaughter who lives here in Morgantown but I live 25 miles across the state line and she has called me and told me she saw it in the paper and I've always wanted to try water aerobics and so we decided we'd do this together; she ended up in the control group but I love it. But I can't swim a lick, but I love it and after I got comfortable and turned the wall loose. I love it and really enjoy it.

John: I need the exercise. I had never tried water aerobics before. I had looked at Sonja Bearce sign for water aerobics at WVU for years and never signed up for it, but Lori's grandmother goes 
to the same senior center I go to and she brought in a bunch of Lori's posters and she talked everybody into it if they had an interest - she's a lot like Lori, a swimming pool full of alkaseltzer, in it so that's why I'm here. I needed the exercise as I said, but I wanted to get a jump start by being forced into doing it 3 times a week because, you know, you sign up for a study and you don't want to let Lori down, so you have to keep coming.

Bob: Bob...I didn't see it but I heard about it through my wife. My wife works at the Health Science Center and she knows Lori. Lori was telling her about it, that she had this program coming up doing her thesis for her $\mathrm{PhD}$ and was wondering if I would be interested to do water aerobics for 3 day s a week. I said well sure, I just retired at the end of January and thought instead of sitting at home on the couch I might as well do something. I'm not much of a self motivated person but when something comes up I'm going to do it so I joined it and I've really enjoyed it and like I said it's getting me off the couch and I'm out here doing something. I've enjoyed it so much I hope to continue it, really. So I'm thinking about joining the HPL and continuing the water aerobics; I really like that because it's a low impact, you know with arthritis and stuff high impact stuff is hard on you. I'd like to continue it too, so I've enjoyed it very much.

Lisa: I'm a teacher and I retired in June and it was a really good way to start in September and be like really happy that I wasn't in school, but have discipline in my life and I exercise a lot anyway so this was just perfect. It came at the right time.

$* * * * * * * * * * * * * * * * * * * * * * * * * * * * * * * * * * * * * * * * * * * * * * * * * * * * * * * * * * * * * * * * * * * * * * * * * * * * * * * * * * * * * * * *$

Jim: Well thank you, everyone. So, I have some questions that I'll be asking from a script and we also have the same question on these that I'll hold up for each question to help us focus on that question. And we're hoping to get as much as possible each and everyone's individual input. So, all of the information and ideas that you offer today are very important to me, and of course, Lori. So I'm encouraging you to share your thoughts, experiences, and ideas freely and all your comments will remain confidential. Feel free to speak up any time, but please speak one at a time and allow others to finish their comments before interjecting. You are free to address one another or a moderator. I would like to keep this casual and conversational. All comments are welcomed, both positive and negative. The important thing is to really get your truthful feelings, how you really feel. Now this session will go on for no longer than one hour. We don't have a scheduled break but please feel free to help yourself to more coffee and so on, and if you need to use the restroom it is right out the hall to the left. Ok, so the primary purpose of this meeting is to see how you feel about the aquatic exercise sessions. Any barriers you encountered, participation, and the effects it had on your lifestyle now that the study is over. I'd also like to know how you relate exercise to your cognitive abilities, as well as your candid thoughts on the project as a whole. Please keep in mind there are no right answers. All comments are welcomed and will help give insight to allow the recorder and to me to make adjustments and improvements to the design of future studies. All comments will remain confidential. I'm also asking you please to not share 
outside of this group something that another participant says by name. For example, John Doe says he has no social support from home. Ok, any questions before we start? Alright. So, our first question is: Now that you have completed the program, how do you feel about exercise?

Lisa: It gives me energy.

Bob: It's a necessary part of everyday life. I've seen my parents and my mom was too sore to move around \& she doesn't move around, and her joints are all frozen up now so I just think it's a necessary part of life.

John: I think from my observation that people are getting along better physically and mentally and as they get older people who have created their own or joined exercise programs and I think I would do well to do the same.

Susan: I know exercise has always been good for my medical conditions, but that was not enough to actually get me out and exercise. I have 2 or 3 different machines at home, but I would never get on them. Since I've been doing this water aerobics and I saw those steps out there and knew there were more steps in here I thought oh my God I'm never going to make it up those steps because of my knees, but I had no trouble with my knees at all coming up all of those steps. I know how important it is.

Betty: Well I feel like the exercise keeps me more flexible and its real good and I like it. I am walking and I feel real good \& much better

John: For medical conditions my doctor said you would do well to lose 30 pounds and you need to do some exercise. And um, so I thought this was a good start, but he always says in order to get the ass you want, you have to get off the ass you have. (Laughter from group)

Pam: I agree with pretty much everyone else. The exercise gives me more energy and flexibility and it also makes me feel better about myself knowing that I'm taking control of my own body and doing what is good for it, so...

Jim: Anybody else have anything else they'd like to say in terms of how you feel about exercise now that the program is over?

Lisa: It's easier to be motivated when you're with a group of people.

Pam: I agree - lots of agreeing from group***

$* * * * * * * * * * * * * * * * * * * * * * * * * * * * * * * * * * * * * * * * * * * * * * * * * * * * * * * * * * * * * * * * * * * * * * * * * * * * * * *$

Jim: Thank you. Well, we'll move on to the next one. Do you think you will continue to exercise now that the program is over? 
***multiple people said yes all at the same time

Lisa: She said she'd give us a list of what's available in town that does stuff in the water so I do hope she does give that to us because I would pay to continue a class.

John: My comment was going around the same sort of thing. It's nice exercising and not getting all sweaty but being in a whole swimming pool full of sweat. And I would like to continue on with some sort of water exercise.

Jim: I heard and saw a few of you say yes and nod your head. Does anybody else have anything they'd like to add to this...

Bob: I plan to continue, but I'm not much of a self motivated person. So, something structured like this will help me because I hope to get involved in something else in the HPL. The only thing active I did before this was walking the dogs, and the dogs have back problems (laughs) so they don't walk very far and I don't get out and walk much on my own without them. I need something structured I guess. I hope to continue and just motivate myself to do it more. Like I said, it's an important and necessary thing to continue.

Patty: I'd like to continue to exercise. I more or less joined the class for motivation because I had always walked - I walked to school, I walked to work, I've always walked. And this is personal, but when I lost my husband...I had to take care of him for a few years and so I got out of the...continuing to walk, I just couldn't get out and walk and then going to an empty house, that's where it all just stopped. I did other things, but I didn't exercise. So I'm using this as a motivation to get myself back, hopefully

Pam: I have to agree with other people too. You know, feeling that you are obligated because you sign up, and if you sign up to do something you should do it even though some days you really don't want to go but you don't want to let Lori down. I have a group at my church that exercises and that's kind of how we motivate each other too; we don't want to let the other one down. So I think group exercise helps us.

Jim: Thank you. Anybody else?

Susan: I want to be able to continue water aerobics and I'd pay to continue. It's not...I don't feel like I'm exercising. I know I'm doing something good for my body, but it's not strenuous to me exercise is strenuous, to be exercising in the water. And since I've retired I don't get out of bed for anything. And I look forward to Monday, Wednesday, and Fridays to come into this class. I wake up several times during the night to make sure I didn't miss my alarm going off. So I don't feel like I'm exercising, but I know I'm doing something good for my body. So I would love to be able to continue it. And I've paid gym memberships, I've done curves, I've done everything and it's just strenuous, it's just hard work and I'm lazy (laughs). 
Lisa: I agree with Susan. I like an exercise that's fun. I do Zumba and I don't even know that I'm exercising. I'm just dancing and having a good time, and this feels like I'm having a good time.

Susan: And all the people you are with have a lot to do with it to and all of these people are so nice. I enjoy coming and talking with them. I hope to be able to continue this; I really enjoy it.

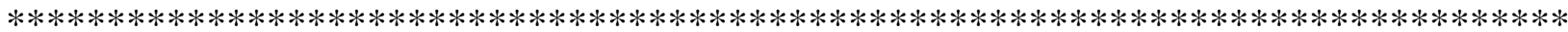

Jim: Thank you, Susan and Lisa. Well, we'll move on to the next one then. So for some of the questions that I will ask you will have already previously said something that really is the answer to the question. If there's something you want to add when the question comes up, feel free to do that. Ok, so if you do plan to continue, what type of exercise do you think you will participate in?

Lisa: I would love to do some water.

John: Again, I like the effect of buoyancy. It reduces the strenuousness of the exercise and it's something that you can do for an hour straight.

Bob: I hope to continue with the water aerobics too... like I said with the HPL. Plus I'd like to get back into walking, now it feels easier to do. I'd like to do continuous water aerobics and more walking.

Jim: Would anyone else like to comment on what type of exercise they will do?

Betty: I will continue to do some walking \& yoga. I would like to do the water exercise, but my skin is really messed up and sensitive and the chemicals in the water make me itch all over and it is very uncomfortable. So I probably won't be doing water aerobics which makes me sad because it was fun, but it makes me itch.

Patty: Walking.

Pam: Mostly walking, although I would probably participate in another water aerobics class. But walking is my main form.

$* * * * * * * * * * * * * * * * * * * * * * * * * * * * * * * * * * * * * * * * * * * * * * * * * * * * * * * * * * * * * * * * * * * * * * * * * * * * * * * * \pi$

Jim: Ok, well the next question here deals with support. So, what types of support do you think will help you keep exercising?

***many people stated being part of a group

Bob: Group participation, trying to get self motivated. 
Lisa: The one thing that helps me continue to exercise is walking, but I walk alone. I'd love to walk with somebody but all my friends are non ambulatory. So, I listen to that and I get out there and walk hills. But that does help me.

$* * * * * * * * * * * * * * * * * * * * * * * * * * * * * * * * * * * * * * * * * * * * * * * * * * * * * * * * * * * * * * * * * * * * * * * * * * * * * * * * * * *$

\section{Jim: So how do you think exercise affected your cognitive function: thinking processes, remembering, learning?}

Patty: I haven't seen any change.

Lisa: I'm anxious to see the results of the tests... am I going to remember these things from the last time, I don't know. But I do a lot of other things too like puzzles, reading a lot, and I think when I was teaching I had to remember so many things so I don't know whether my brain is leaving yet or not. (laughs) But I forget names all the time.

Jim: Does anyone else have any further comments on exercise and how it affected your remembering?

Bob: I thought that that was the main focus of this study, wasn't it? How it affects your cognitive function? I don't see where it has helped.

***many people stated hopefully when we take the test we will be able to see the results

Lisa: I'd like to say yes, it really has helped me but I really don't know.

Pam: You know, I'm not sure, but it makes me feel better about myself that I am doing something proactive. And I'd like to think that getting more blood flow and oxygen to my brain is helping, but I guess the test will tell.

Lisa: I thought that when she would say "do this, and now do this, and oh do this now", that she was trying to pounder our brains. But we'll see if it worked. I'd get it figured out then she would go to something else...group laughing

Jim: So you have mentioned some examples already but let me ask you, do you have any personal examples since you started this program of ways that exercise affected your cognitive function, or not really?

Lisa: Well, I feel more alert for the rest of the day so I think that helps.

Pam: I agree that the energy level and alertness improved.

$* * * * * * * * * * * * * * * * * * * * * * * * * * * * * * * * * * * * * * * * * * * * * * * * * * * * * * * * * * * * * * * * * * * * * * * * * * * * * * * * * * *$

Jim: Well that takes us into the next question: In what ways does exercise affect your daily activities? 
Lisa: I feel like there is a sense of accomplishment already. It's at the beginning of the day, so buddy now I can do anything.

John: When I first started doing this even though I did notice that Lori was taking us in a progression getting more difficult and more difficult each time we came out there. When I first started doing this I needed some recuperation time that I needed to take, but now my day is easier.

Jim: Anybody else have any comments of how exercise affected your daily activities?

Pam: It got me out of bed earlier. I was definitely up 1.5 to 2 hours earlier than I normally would have been, so it got me moving quicker.

Bob: Being that it is structured and within the classes, it has got me up moving earlier that I would, so I guess it provides structure within my life.

Jim: How about changes in your daily activities...how do you think your daily activities have changed since you've been exercising?

Lisa: It coincided with my retirement so I don't know what it would have been like. I got up yesterday at 7 and went on a walk, but I would have still done exercise. This made it easier because someone else provided the exercise for me \& I just let my body do it.

Jim: Anybody else have any comments of how your daily activities have changed since you've been exercising?

Betty: I think that because I have more energy I can do more stuff during the day than I could before...accomplish more.

Susan: It gets my day started earlier with energy rather than sitting the whole morning in front of the TV with a cup of coffee and not being able to really get myself into gear until noon or 1 o'clock. When I leave my exercise class and go home I'm ready to do stuff other than sit in front of the TV. Very seldom do I put my TV on anymore. You know, I do a lot of reading but usually I'm doing something piddling around the house, and when the weather was warm I was outside doing things out in the yard. It has made a difference in the way my day starts.

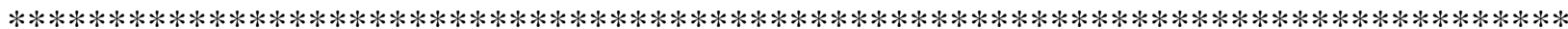

Jim: Now, in the beginning we shared comments about why we joined the program, but we have a question specifically on that. What motivated you to come to the aquatic exercise classes?

Lisa: It was free.

Pam: Yes, that was very helpful. (LOTS OF AGREEMENT FROM GROUP) I have a problem, I guess, paying money to do exercise when there is free exercise available such as walking. But 
with something free like this, and it was different. I had never done and I had heard people say it was a lot of fun, so I thought this was my opportunity as well as to support my cousin.

Patty: First thing, it was free. Second thing, I feel like I was helping to find out if water exercise would help cognitive function...helping Lori, helping in some way.

Susan: I've always wanted to try aquatic exercise, but I'm like those ladies...I didn't want to pay for something because I'm good for paying for memberships and not sticking to them. And when I found out it was free I jumped on the opportunity. I had been wanting to try it.

Patty: Seeing it in the newspaper offered through the University, but that caught my eye...seeing it in the newspaper was a factor for participation...

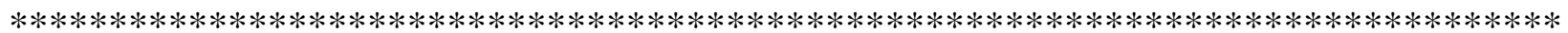

\section{Jim: So what did you like best about the aquatic exercise program?}

Lisa: Just how you feel afterwards. It is kind of energizing.

Pam: I like the fact that I can do the same kind of exercises that I can do on ground in the water and I can use more exertion and have less effect on my joints, I mean I could get the exercise I needed but it didn't hurt or it wasn't as hard to do as it would be if I was doing the exercises on the ground.

John: I think the heart rate monitors that we used in the aquatic exercise program really helped. It helped us figure out how much exertion you were putting in to the exercise. That was great input. Each time we were asked, "What's your heart rate? How hard are you working?"

LAUGHS - it was immediate feedback

Pam: Yes, but sometimes I felt like I was working really hard and the heart rate monitor said I wasn't. Laughs

John: Same thing here, so sometimes the heart rate monitors were faulty. Yea, it was the HR monitors...LAUGHS

Betty: I also liked the fact that the exercise is much easier for your joints and everything because whatever exercise I do out of the swimming pool is much harder on my joints and it was really much, much easier in the pool.

Susan: I like the fact that the class was structured that Lori and the girls and the guy were there telling us what to do and constantly kept us moving... because if I had to do it on my own I probably would have periods of time when I would do nothing, but they kept me moving and I like the fact that they were there and had it structured. 
John: Another thing about the pool, if I was in the middle of some movement and I miscalculated, instead of going down and busting my rear I had a chance to correct because I was less heavy that's that buoyancy thing.

Patty: I agree with all of the above and it was fun.

Bob: I think if anything would have come up I would have been anxious to join an exercise program but I just so happened to hear about this and it's a lot easier on the joints. It's a good starting point, I guess to start out in the water with the buoyancy factor. But any kind of exercise is good...just gotta get yourself self motivated. This was a great start.

Patty: She was encouraging. She always said, “That's good! Keep it up! Keep going!” And you just felt like you had to because you wanted to do it for her too...laughs...

$* * * * * * * * * * * * * * * * * * * * * * * * * * * * * * * * * * * * * * * * * * * * * * * * * * * * * * * * * * * * * * * * * * * * * * * * * * * * * * * * * * * * * *$

Jim: Well let's switch it up here a bit and talk about what discouraged you from going to the aquatic exercise classes?

John: WVU closing the parking lots.

***short, off topic discussion about parking! EASE \& ACCESSIBLITY OF EXERCISE WAS explored here in an off-topic discussion....this was a barrier!

Lisa: I substitute teach and would get a call and I would say no I can't come in because I have to go to this class...I would substitute teach, but never missed a class for it. I did sacrifice a few pay days for this program because I didn't want to let Lori down. So if I did it again \& she didn't need my HR \& need the data \& I didn't want to let her down. If I sign up later I will definitely go in for work and miss a class but I did sacrifice that for this...I made this a top priority.

John: If there were three like 8-9am, 1-2am, and 7-8pm we wouldn't have to miss anything.

Lisa: but it really was flexible because sometimes I would work in the morning and then I could go to the 1:00 - that was nice.

$* * * * * * * * * * * * * * * * * * * * * * * * * * * * * * * * * * * * * * * * * * * * * * * * * * * * * * * * * * * * * * * * * * * * * * * * * * * * * * * * *$

Jim: I just want to say, please be frank with the next question. We really want to know, so it's not going to hurt anybody's feelings. So, what did you like least about the aquatic exercise classes?

Pam: The only thing that comes to mind is that cold jolt when you first get in.

Lisa: The object was to wear the heart rate monitor and they would say "come on, get your heart rate up". But then they would give us exercises that didn't...like the rocking horse, well that didn't do a thing to get my heart rate up. So some of the exercises worked $\&$ they were fun but 
the didn't increase the HR. Another thing is, we would do something really hard and my heart rate would be really high and then they finally get around to me and my heart rate had already gone down. So that kind of tension for me, I mean the heart rate monitor was good but at the same time it made me more tense. And one more thing... for some reason my heart rate seemed like it had to be higher than other people \& I don't understand the mechanics of that but I wish someone would explain - on your goal HR is... and I wish they would explain the reasoning behind that. I didn't know if I was letting someone down if it wasn't in the range it was supposed to be in. Here's my recommendation: tell someone what their heart rate is supposed to be AND why.

Susan: And on the other hand, mine seemed to be lower than everybody else's. I felt like I was working really hard, but I'm on like 5 different blood pressure medicines so I'm assuming that has something to do with slowing me down. I'm hearing people say 100 this, 100 that, and I'm saying 89, 90. Then they would ask how hard we were working and I thought I was working hard but evidently I wasn't.

John: I agree with what Lisa said. But you could add some kind of telemetry heart rate broadcasting that shows up on a screen (LAUGHS)... wouldn't have to wait for someone to come around to you. I take (name of medicine...I think Toporol) which limits the number of beats I can put out per minute anyway and I have no control over it once I take that medication. I offered to go off of it for this, but Lori said NO! (Laughs)

$* * * * * * * * * * * * * * * * * * * * * * * * * * * * * * * * * * * * * * * * * * * * * * * * * * * * * * * * * * * * * * * * * * * * * * * * * * * * * * * * * * * * * * * *$

Jim: Anything else that anyone would like to share about what they liked least about the aquatic exercise program classes? $* * *$ no answer*** Ok, how about barriers. What were the barriers you encountered during this program?

\section{WVU parking brought up again....}

Pam (and two others...probably nodded their heads): I can't think of anything...no barriers.

Bob: Like I said, the program was free so I can't really complain. If I was paying and ran into some of the parking problems I might have a bigger complaint, but other than that there were no barriers.

Jim: Ok, well maybe the next question isn't relevant then but I will throw it out there. So, what solutions and strategies did you come up with to deal with these barriers?

Lisa: Well for me, sometimes I get a sub job just for in the morning or afternoon, so the strategy to get around it was she said if you can't come in the morning you can come in the afternoon. And that was a good solution. It wasn't like if you're in the morning you can only come in the morning. There was the alternative if you had time in the afternoon and that was a good solution to a potential problem. 
Others agreed $* * *$

\section{Jim: So did the barriers change throughout the program or did they remain the same?}

Susan: My only barrier was I had to miss two of the classes for babysitting...my daughter and son in law decided they wanted to go to FL while this was going on. I spent 5-days 24/7 babysitting which was 2 of my days - nothing I could have done about that.

***many said stayed the same...no barriers

$* * * * * * * * * * * * * * * * * * * * * * * * * * * * * * * * * * * * * * * * * * * * * * * * * * * * * * * * * * * * * * * * * * * * * * * * * * * * * * * \pi$

Jim: Who Supports your decision to participate in exercise?

$* * *$ many stated family

Lisa: Self motivated. I like the bragging rights to tell my kids!

Pam: I am pretty much self motivated. I mean my family doesn't usually say ' well I don't think that you should do that' but they don't know the other way either... 'that's great mom' - 'well whatever you want to do mom'

Patty: I'm glad I was participating. I had full support - they were glad to get me out of the house - not that they lived with me - but they were glad I was out.

John: My sister hyper supports this...because as we speak, her husband is lying at home because of diabetes with his legs both removed above the knee, he's on dialysis, but had a heart attack and was 60/40 and now cant go up $1 \%$ solution with dialysis so - his struggle....if anyone ever gets diabetes, you need to do exactly what the doctor says to do because nothing ever pertained to $\mathrm{Jim}$, this is not for me...

Lisa: so your sister's really happy for you

John: Oh, oh yeah.

Bob: I haven't seen anything but support...from the other participants and the students. Wife, students, participants...nothing but support. It's a good program.

John: One of the good things is that the students would come around and ask what's your HR? and even if it dropped off from 107-97 because we changed exercises to something more cerebral they never were judgmental in the way they looked at us or....

Pam: They were kind, weren't they (laughs)

John: They did a good job. 
Pam: I have to say too that in regard to the students and Lori. I did not hear sweetie, dearie you know, that kind of thing. They treated us as people - not as little old people...laughs

Susan: They made me feel comfortable, they really did - always smiling \& happy...not 'oh, another day'. None of em they were always happy. That helped us too because....

***multiple people said students and Lori had a cheerful attitude

\section{Jim: How have they supported you while you are doing this program?}

Pam: Words of encouragement \& talking to me telling me it was really great I was joining something, getting out, exercising.

Lisa: And it was a very nice facility to go to, I mean we had showers, locker rooms, I mean we're blessed to have - many communities don't have something like that. I was really glad, you know its been here for years but being a member of the community and not the university I could never come. So I was like so excited that I could come. Like on...'sacred ground', otherwise you can't help...

Jim: are you saying that the nice facility was a support?

Lisa: I think it was...I was grateful for it.

Patty: When I called I told my family, my son and daughter - they were excited but then they said, how long does it last? And I said well, we'll we will finish up mid of November - mom are you sure you want to do this? And I said yes, I do, cause I think they were proud of me for the fact that I have continued to...I'm $86 \ldots$ and they were glad when I finished they said how you doing, and I said I'm doing fine - hang in there mom \& that's how they supported me during this. After the first couple of weeks they asked 'are you the oldest?' so I asked Lori - quietly(LAUGHS) and she said yes. I'm sure when Friday comes I'm going to get a pat on the backI'm really proud of myself.

$* * * * * * * * * * * * * * * * * * * * * * * * * * * * * * * * * * * * * * * * * * * * * * * * * * * * * * * * * * * * * * * * * * * * * * * * * * * * * * * * * * * * * * *$

Jim:

Betty: I'm just glad I did this whole thing \& thanks for the opportunity.

***LOTS of agreeing \& thank you's from the group

Lisa: I pray it helped Lori, first off, but it really helped us immeasurably.

John: In the early 80 's they had a community outreach program - there were 100 family tickets or cards that you could get into the natatorium when nothing else was going on and I wouldn't mind seeing them reinstate that! It would also be great - since we don't have an indoor 
community pool - I think it would be nice to have a nice pool like that WVU could use for on and off periods.

Lisa: Lori is a professional at everything she does (several nods), in her own self-discipline, class, how she treats her students and us...it's just been inspiring.

John: She would be giving us a recipe - and in the middle yell UP-UP-UP-UP then go right back to the recipe (laughs)

Lisa: where she gets her energy?

Susan: Thankful and grateful for the opportunity to be able to participate in this and get to know Lori and the girls and all - they always had time for you if you had a question it never seems like they were in a hurry and I know that they had other things to do but they would take time to answer your question and you just felt so comfortable with em.

Bob: Grateful for the opportunity.

John: A lot of nice group mates.

Susan: I can't wait to get measured. 


\section{Appendix J: Triangulation Matrix for RQ1}

Table Matrix on Qualitative and Quantitative Data for Cognitive Parameters in the Intervention Group

\begin{tabular}{|c|c|c|c|c|c|}
\hline \multirow{3}{*}{$\begin{array}{l}\text { Cognitive Parameters } \\
\text { OTS: Problems Solved in the } 1^{\text {st }} \text { Choice }\end{array}$} & \multicolumn{5}{|c|}{ Quantitative Findings $(n=17)$} \\
\hline & \multicolumn{2}{|r|}{ Pre } & \multicolumn{2}{|c|}{ Post } & \multirow[b]{2}{*}{$\mathrm{P}=0.260$} \\
\hline & 15.25 & $\mathrm{SD}=2.52$ & 15.75 & $\mathrm{SD}=2.60$ & \\
\hline $\begin{array}{l}\text { OTS: Mean Latency to Correct } \\
\text { (seconds) }\end{array}$ & $1.33 \mathrm{~s}$ & $\mathrm{SD}=0.18$ & $1.30 \mathrm{~s}$ & $\mathrm{SD}=0.20$ & $\mathrm{p}=0.170$ \\
\hline $\begin{array}{l}\text { OTS: Mean Latency to First Choice } \\
\text { (milliseconds) }\end{array}$ & $\begin{array}{l}13089.05 \\
\mathrm{~ms}\end{array}$ & $\mathrm{SD}=4788.43$ & $11537.88 \mathrm{~ms}$ & $\mathrm{SD}=4803.05$ & $\mathrm{p}=0.0$ \\
\hline PAL: Total Errors & 34.88 & $\mathrm{SD}=24.19$ & 16.29 & $\mathrm{SD}=19.02$ & $\mathrm{p}=0.584$ \\
\hline SWM: Strategy Score & 17.41 & $\mathrm{SD}=3.45$ & 17.12 & $\mathrm{SD}=3.26$ & $p=0.152$ \\
\hline SWM: Between Errors & 19.65 & $\mathrm{SD}=7.76$ & 14.65 & $\mathrm{SD}=8.58$ & $\mathrm{p}=0.014$ \\
\hline AST: Congruency Cost & $188.75 \mathrm{~s}$ & $\mathrm{SD}=99.70$ & $155.00 \mathrm{~s}$ & $\mathrm{SD}=93.59$ & $\mathrm{p}=0.956$ \\
\hline AST: Switching Cost & -106.65 & $\mathrm{SD}=153.21$ & -171.00 & $\mathrm{SD}=122.06$ & $\mathrm{p}=0$ \\
\hline AST: Percent of Correct Trials & $89.15 \%$ & $\mathrm{SD}=7.91$ & $91.54 \%$ & $\mathrm{SD}=8.59$ & $\mathrm{p}=0.521$ \\
\hline RTI: 5-Choice Reaction Time & $379.30 \mathrm{~ms}$ & $\mathrm{SD}=76.63$ & $374.16 \mathrm{~ms}$ & $\mathrm{SD}=67.96$ & $\mathrm{p}=0.912$ \\
\hline RTI: 5-Choice Movement Time & $466.33 \mathrm{~ms}$ & $\mathrm{SD}=87.67$ & $471.46 \mathrm{~ms}$ & $\mathrm{SD}=85.13$ & $\mathrm{p}=0.309$ \\
\hline \multicolumn{6}{|c|}{$\begin{array}{l}\text {-I think it keeps you in shape, not only physically but even mentally } \\
\text {-it has to do something to your whether you realize it or not. It has to. It has to affect the whole body, your wh } \\
\text { cognitive function] } \\
\text {-well, I guess I was struggling before [cognitively] and I was hoping it would help me. I stumble for words son } \\
\text { stumble remembering things, and I'm afraid that some of the things I've done have been worse. And I struggle } \\
\text { different steps... I know I've made even bigger mistakes while driving. You know, I got out of the car at the gro } \\
\text { running. See, I really am struggling. But my husband says you may not have improved, but it could have been } \\
\text { know exercise has endorsed me very good emotionally. I was very depressed when I started and I'm much bett } \\
\text { there is a lot of benefits to it, but I feel like it's important to keep trying even if you aren't getting the results yo } \\
\text { have gotten* } \\
\text {-my coordination isn't the best in the world, and I find by exercise or repetition like we did in class that I saw a } \\
\text { end of the class than the beginning... at the end I felt that I was much better in following } \\
\text {-I was thinking in struggling to do the steps, I was helping my brain make different paths.-I think from my obse } \\
\text { getting along better physically and mentally and as they get older people who have created their own or joined } \\
\text { I think I would do well to do the same } \\
\text {-I haven't seen any change [relating to cognitive function] } \\
\text {-I don't see where it has helped [relating to cog fx] }\end{array}$} \\
\hline
\end{tabular}


-I'd like to say yes, it really has helped me but I really don't know [cog fx]

-I'd like to think that getting more blood flow and oxygen to my brain is helping, but I guess the test will tell

-I thought that when she would say 'do this, and now do this, and oh do this now' that she was trying to pound o figured out then she would go to something else

-I feel more alert the rest of the day

-I agree that the energy level and alertness improved

-my husband, he suffers from dementia some \& I think the reason he pushed me into this was because if I got it 1

the end. Between the two of us there is 1 of us.

*negatively associated comment 


\section{Appendix K: Triangulation Matrix for RQ2 \& RQ3}

Table Matrix on Qualitative and Quantitative Data for Wellness Parameters in the Intervention Group

\begin{tabular}{|c|c|c|c|c|}
\hline \multirow{2}{*}{$\begin{array}{l}\text { Wellness } \\
\text { Parameters }\end{array}$} & \multicolumn{3}{|c|}{ Quantitative Findings $(\mathrm{n}=17)$} & \multirow{2}{*}{$\begin{array}{l}\text { Qualitative Findings }(\mathrm{n}=14 \\
\text { Participant Comments (Frequency: } 35\end{array}$} \\
\hline & Pre & Post & p-value & \\
\hline Distance Walked & $458.98 \mathrm{~m}$ & $\mathrm{SD}=106.35$ & $\mathrm{p}=0.177$ & \multirow{6}{*}{$\begin{array}{l}\text {-it gives me energy } \\
\text {-I had no trouble with my knees at all coming up } \\
\text { [steps leading to FG room] } \\
\text {-gives me more energy and flexibility and makes } \\
\text { myself } \\
\text {-I see the difference I can do things I couldn't do } \\
\text {-I found it quite fruitful and helpful for me...I ha } \\
\text { movement...it's been easier for me to get up } \\
\text {-it made my knee better, I don't have to wear my } \\
\text {-I am walking now and I feel real good \& much } \\
\text {-now it [walking] feels easier to do } \\
\text {-when I first started I needed some recuperation } \\
\text { easier } \\
\text {-I have more energy I can do more stuff during t } \\
\text { before... accomplish more } \\
\text {-gets my day started earlier with energy } \\
\text {-I noticed a difference on the stairs for me. I can } \\
\text { top [of the stairs] and don't have to stop and can } \\
\text {-my knees are a lot better too } \\
\text {-my knees feel better too } \\
\text {-I've increased my daily activities } \\
\text {-I can take the stairs one step at a time...but I co } \\
\text { beginning [of the program]. } \\
\text {-going from depending on a [knee] brace to not } \\
\text {-raking leaves to shoveling snow...easier to ben } \\
\text { class } \\
\text {-I don't have as much lower back pain as I did b } \\
\text { program] } \\
\text {-I have muscles for the first time in my life. } \\
\text {-I've lost weight, feel good about myself } \\
\text {-It does help you to do your activities and everyt } \\
\text {-it keeps you in shape } \\
\text {-gives you more energy throughout the day and } \\
\text { better an awful lot too } \\
\text {-improves your mood too (group agrees) } \\
\text {-for the first month, I was so dead tired by the tir } \\
\text { that you just went to sleep....I don't even nap at } \\
\text { energy is so much better. }\end{array}$} \\
\hline RHR & 63.71bpm & $\mathrm{SD}=7.24$ & $p=0.841$ & \\
\hline Resting SBP & $128.71 \mathrm{mmHg}$ & $\mathrm{SD}=18.96$ & $p=0.462$ & \\
\hline Resting DBP & $69.29 \mathrm{mmHg}$ & $\mathrm{SD}=9.59$ & $\mathrm{p}=0.014$ & \\
\hline Body Weight & $185.941 \mathrm{lbs}$ & $\mathrm{SD}=45.74$ & $\mathrm{p}=0.289$ & \\
\hline $\begin{array}{l}\text { Body } \\
\text { Composition }\end{array}$ & $40.67 \%$ & $\mathrm{SD}=7.64$ & $\mathrm{p}=0.560$ & \\
\hline
\end{tabular}




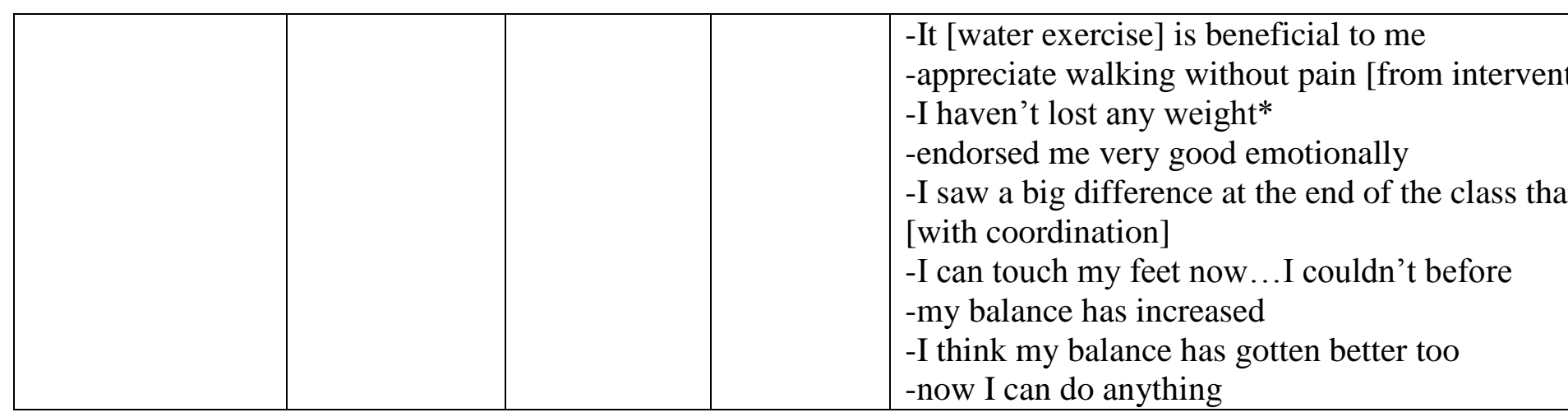

*negatively associated comment 


\section{Appendix L: Triangulation Matrix for RQ4}

Table Matrix on Qualitative and Quantitative Data for Psychosocial Mediators in the Intervention Group

\begin{tabular}{|c|c|c|c|c|c|}
\hline \multirow{3}{*}{$\begin{array}{l}\text { Total Barriers } \\
\text { (excluding } \\
\text { barriers to SE) }\end{array}$} & \multicolumn{5}{|c|}{ Quantitative Findings $(\mathrm{n}=17)$} \\
\hline & \multicolumn{2}{|r|}{ Pre } & \multicolumn{2}{|c|}{ Post } & p-value \\
\hline & $\begin{array}{l}2.1 \\
1\end{array}$ & $\mathrm{SD}=0.59$ & 2.09 & $\mathrm{SD}=0.38$ & $\mathrm{p}=0.918$ \\
\hline
\end{tabular}

Qualitative Findings ( $=14)$

\section{ACCESSIBILITY:}

-I would like to do the water exercise, but my skin is really messed up and sensitive and the chemicals in the wat over and its very uncomfortable. So I probably won't be doing water aerobics which makes me sad because it w me itch.

-WVU closing the parking lots [discourages me from coming] LOTS of agreeing from the group -the cold water [discourages me from coming] - GROUP LAUGHS - more of a big joke

-It was hard to park sometimes

-parking was the worst

-traffic because I live on the other side of town \& one day I sat in traffic for $30 \mathrm{~min} . .$. no point in going $30 \mathrm{~min}$ la AGREES

-cold jolt when you get in [in reference to what they liked least about the program]

-didn't like when I had to get both my fee up off the floor. I didn't like it. I don't like it...I'm just not comfortab water to let myself do it and go under. That's my fear.

-I'm the same way [fearful] because I can't swim. So when my feet leave the ground I have fear.

-Parking! [in reference to barriers encountered]- GROUP PROCLIMATION

-traffic or parking

-they should do something about parking

-if they ever get this parking at WVU straightened out the world will probably be ending soon

-since we don't have an indoor community pool, I think it would be nice to have a pool like that WVU could use periods

-it was free - yes, that was very helpful - GROUP AGREES

-my insurance doesn't pay for it, but you're on a fixed income...

\section{SUPPORT:}

-my only disappointment with the study is that my husband got in the control group

-I'm pretty much self motivated, I mean my family doesn't usually say ' well I don't think that you should do the know the other way ' that's great mom' - 'well whatever you want to do mom'

-when I told my family, they were excited but then they asked, how long does it last? ... are you sure you want to -I'd love to walk with somebody but all my friends are non-ambulatory

\section{FEAR OF FAILURE:}

-I didn't want to pay for something because I'm good for paying for membership and not sticking to them -we would do something really hard and my HR would be really high... when they finally got to me it had gone tension for me...made me more tense

-my HR seemed lower than everyone else's...I thought I was working hard but evidently I wasn't [referring to th -I didn't know whether I should just keep moving to keep the HR up...or trying to do the steps

-for some reason my HR had to be higher...I wish they would explain the reasoning for this...tell someone what to be and why 
-all of us as aging $\&$ kind of forget that you can't do a whole lot of things if you don't have the real resolve... -but at my age, if I exercise too much I will end up in a chair

\section{PRIORITIES:}

-life gets in the way sometimes...stop here or there and then the day is over

-I had to miss 2 classes for babysitting...nothing I could have done about that

-we were gone for a while on vacation

-I was gone on that trip for a week

-I had to go to VA to settle up an estate problem

-life happens

-commitment... although its early...even though its hard some mornings

-and if it's a cold, wet, foggy morning, covers feel much better in your bed than getting out, but I know I have to -if there were like 3: 8-9, 1-2, and 7-8 we wouldn't have to miss anything

\section{HEALTH STATUS}

-buoyancy. It reduces the strenuousness...something that you can do for an hour straight

-dependent on a brace

-low back pain when I started

-can use more exertion and have less effect on my joints. I could get the exercise I needed but it didn't hurt or it

as...on the ground

-a lot easier on the joints... buoyancy factor

-I couldn't come a couple of times... sick

-I had some health problems that discouraged me too...dental

-she had some dental and I had bronchitis. I was ill the last couple of weeks and that was certainly a barrier

-with arthritis and stuff, high impact is hard on you

-I have a lot of arthritis, so I find it difficult to try on land

-... arthritis starting and I've found that ...I've had more movement

-I've got extensive arthritis so I just quit doing anything...I hurt my knee badly 2 yrs ago and I wasn't able to do -having problems with my balance

-for a while especially on the ground it [balance] was rough

-the exercise is much easier for your joints \& everything because whatever exercise I do out of the swimming po my joints and it was really much, much easier in the pool

-if I was in the middle of some movement \& miscalculated, instead of going down...I had a chance to correct

-it's easier on the joints

\section{BARRIER REMOVAL:}

-Just retired

-retired in June

-keeps me more flexible...feel much better

-she said she'd give us a list of what's available

-nice exercising and not getting all sweaty

-we don't want to let the other one down

-when I first stated...problems with my balance... water might help

-I'd like to get back to walking, now it feels easier to do

-feel more alert for the rest of the day

-energy level and alertness improved

-coincided with my retirement so I don't know what it would have been like

-much easier on the joints

Quantitative Findings (n = 17) 


\begin{tabular}{|c|c|l|l|l|c|}
\hline \multirow{2}{*}{} & \multicolumn{2}{|c|}{ Pre } & \multicolumn{2}{c|}{ Post } & p-value \\
\cline { 2 - 6 } & 4.60 & $\mathrm{SD}=1.11$ & 4.57 & $\mathrm{SD}=0.87$ & $\mathrm{p}=0.323$ \\
\hline
\end{tabular}

Qualitative Findings $(n=14)$

HEALTH/ADLs:

-...'Sick, but I did think moving was better than not moving'

-feel better about myself that I'm doing something proactive

-when I first started I needed recuperation time...now my day is easier

-balanced increased...leaned to the left before

-my balance has gotten a lot better too... and my knees are better too

-my knees are better too...my knees have not been bothering me as much

-I have muscles for the first time in my life

-I have increased my daily activities doing this

-I can take stairs one step at a time...I couldn't do that at the beginning

-going from depending on a brace to not having to... big plus

-we went from raking leaves to shoveling snow, so I had to do a little of both. I found it a little easier to bend an -more energy... do more stuff...accomplish more

-I can get my foot up under my chin in water, but on land I can't even get up to my waist. So just being able to u easier way, a plus for me because if I get down on the floor, its really a chore to get back up...yes, I'm more mol -how you feel afterwards...it's kind of energizing

-I need the exercise [in reference to health concerns]

-it keeps me moving

-I see the difference, I can do things I couldn't

-I've lost weight, feel good about myself

-\& I do have arthritis starting and I've found that...I'm able to have more movement

-I don't have to wear my brace anymore

-I think it keeps you in shape, not only physically but mentally

-gives you more energy throughout the day \& it helps you move better and awful lot too

-it gives me energy

-I know exercise has always been good for my medical conditions

-I'm never going to make it up those steps...but I had no trouble...

-the exercise gives me more energy \& flexibility \& it also makes me feel better about myself knowing that I'm t own body \& doing what is good for it.

-the energy is so much better now

-find that it [water exercise] is beneficial to me

-I do appreciate walking without pain [attributes to water exercise]

-it improves your mood too - GROUP AGREES

-I know exercise endorsed me very good emotionally. I was very depressed when I started and I'm much better $r$ -I've seen my parents \& my mom was too sore to move around and she doesn't move around, and her joints are think it's [exercise] a necessary part of life

-I walk alone, I'd love to walk with somebody but all my friends are non-ambulatory...but that does help me.

-I was struggling before and I was hoping it would help me

-it makes me feel better about myself that I'm doing something proactive

-it's scary because I'm afraid I'll lose it [ability to walk steps]

-my sister hyper-supports this... her husband has diabetes

\section{KNOWLEDGE:}

-her doc recommended aquatic therapy 
-I needed the exercise [doc says]

-my doctor said [to exercise]

-I asked my doc if he thought water might help [with balance] and he said well its worth a try

PROGRAM \& FACILITY ACCESSIBLITY:

-I'm not much of a self-motivated person but when something comes up I'm going to do it

-it came at the right time

-a chance for me to learn some new exercise and what to do

-I joined the class for motivation because I had always walked... but when I lost my husband...I just couldn't get going into an empty house, that's where it all just stopped...I'm using this as motivation to get myself back

-coincided with my retirement

-sounded fun and thought this was my opportunity [exercise]

-don't want to let Lori down so you have to keep coming

-needed a jump start... forced into doing it $3 \mathrm{x} / \mathrm{wk}$

-I needed something structured I guess I'm not much of a self-motivated person

-feeling you are obligated because you sign up \& if you sign up to do something you should do it even though so don't want to

-I pray it helped Lori

-I think its commitment. You set a time and then you do it because you made that commitment

-if you make an official commitment. Its one of the the main things

-and if it's a cold, wet, foggy morning, covers feel much better in your bed than getting out but I know I have to

-commitment to have to be here

-like that the class was structured...telling us what to do

-I sub...would say 'no' because I have to go to this class...I made this a priority

-it was free

-it was free - GROUP AGREES

-first thing, it was free

-I didn't want to pay for something because I'm good for paying for memberships and not sticking to them \& wh free I jumped on the opportunity. I had been wanting to try it.

-the program was free so I can't really complain

-I'm so thankful I was able to get into this program \& the price was definitely right.

-a very nice facility to go to...we're blessed to have

\section{PERCIEVED STATUS LEVEL/PRESTIGE:}

-seeing it in the newspaper offered by the University...caught my eye

-its been here for years but being a member of the community \& not the university, I could have never come. So that I could come. Like on sacred ground

\section{NOVEL/FUN EXERCISE:}

-its nice exercising and not getting all sweaty

-different type of exercise, it keeps me moving

-it was different, I had never done

-I like exercise that's fun

-I didn't even feel like I was exercising... knew I was putting stress on... getting short of breath

-I liked the stretches too, that was a good part of it - almost like yoga

-telling us what to do and constantly kept us moving

-easier to be motivated when your with a group of people

-we motivate each other too

$-\&$ all the people you are with have a lot to do with it [fun] too $\&$ all these people are so nice. I enjoy coming an 
-I love the people

-we did a lot of sharing. Personal lives, that was wonderful

-I wanted to see the people... you all were a big draw for me

-a lot of nice group mates

-you know another thing I enjoyed were her [instructors] recipes

- her [instructors] run, her stories, I's be thinking I'm so tired. I don't think these legs ar going to go any further, riding her bike 150 miles and I'd think Oh, I can do this.

-if she can ride over 100 miles on Sat then I can surely do this

-they all [instructors] put youth back into us

-it was exercise but you really enjoyed it

-The instructors made it fun

-they all did, they were a wonderful group of people [ instructors]

-the young people [instructors] were superb

\begin{tabular}{|c|c|c|c|c|c|}
\hline \multirow{3}{*}{$\begin{array}{c}\text { Barriers to Self- } \\
\text { Efficacy }\end{array}$} & \multicolumn{4}{|c|}{ Quantitative Findings (n = 17) } \\
\cline { 2 - 6 } & \multicolumn{2}{|c|}{ Pre } & \multicolumn{2}{|c|}{ Post } & p-value \\
\cline { 2 - 6 } & 3.82 & $\mathrm{SD}=0.72$ & 3.68 & $\mathrm{SD}=0.75$ & $\mathrm{p}=0$. \\
\hline
\end{tabular}

Qualitative Findings $(n=14)$

HEALTH AND ABILITY LEVEL:

-I haven't lost any weight and my husband is very disappointed in that*

-I struggled very much with the different steps*

-sometimes I felt like I was working really hard \& the HR monitor said I wasn't*

-sometimes the HR monitors were faulty* - LAUGHS

-struggled with coordination \& trying to do the steps*

FEAR OF WATER:

-can't swim a lick, but I love it \& after I got comfortable \& turned the wall lose...

-that was the first time I had ever been in the water...definitely have fear of water...so some of the things, when the floor, you lose me

-definitely have a fear of water. so some of the things, when you take both feet off the floor, you lose me...

-I don't like pools \& I don't swim \& I don't like water

-I didn't like when I had to get both my feet up off the floor...not comfortable enough in water to let myself do i

That's my fear

-I'm the same way [fear] because I can't swim. So when my feet leave the ground I have this fear.

\begin{tabular}{|c|c|c|c|c|c|}
\hline \multirow{2}{*}{ Social Support } & \multicolumn{4}{|c|}{ Quantitative Findings (n= 17) } \\
\cline { 2 - 5 } & \multicolumn{2}{|c|}{ Pre } & \multicolumn{2}{c|}{ Post } & p-value \\
\cline { 2 - 6 } & 3.59 & $\mathrm{SD}=0.71$ & 3.65 & $\mathrm{SD}=0.70$ & $\mathrm{p}=0$. \\
\hline
\end{tabular}

Qualitative Findings $(n=14)$

INFORMATIONAL:

-lori's grandmother goes to the same Sr. Ctr...brought in a bunch of lori's posters and talked everybody into it if -I have a cousin... mentioned that...

-hear about it through my wife

-chance to learn new exercises

-my doctor said

-said she would give us a list of what's available

-hear people say it was fun [water ex]

-got wind of it, she let me know...herd it through the grapevine 
-HR monitors helped us figure out how much exertion

-likes that the class was structured...telling us what to do

\section{INSTRUCTORS:}

-I've enjoyed the young girls[instructors]

-don't want to let Lori down

-She [lori] just encouraged and pushed us to the place where it makes you want to do more

-I need something structured

-I had access to exercise at HW (place to learn)

-made it easier because someone else provided the exercise for me

-with the instructor, she made it fun

-they all did [made it fun]

-HR monitors... helped us figure out how much exertion

-class was structured...telling us what to do and constantly kept us moving

-she was encouraging [lori] you just felt like you had to

-I haven't seen anything but support...from the other participants and students[instructors]

-one of the good things is that the students would come around and ask what's your HR? \& even if it dropped of judgmental in the way they looked at us or...

-in regards to the students \& lori. I did not hear sweetie....they treated us as people, not as little old people

-they [inst] made me feel comfortable - always smiling \& happy - LOTS OF AGREEMENT

-I pray it helped Lori

-they always had time for you if you had a questions... and you just felt so comfortable with them

-that was the great thing a about the program \& lori \& the girls \& everybody, but especially Lori. She encourag that you could do and she accepted whatever limitations you had and she didn't make you feel small...or funny want to go because I'm the dunce of the class... she accepted us as individuals.

-here aids were good too

-instructors actually got in...it was nice...you didn't think it was above them

\section{FAMILY:}

-GROUP STATED FAMILY AS SUPPORTERS

-I had full support [family] - they were glad to get me out of the house...they were just glad I was out

-my sister hyper-supported this...her husband had DM...

-I haven't seen anything but support...wife

-words of encouragement \& talking to me telling me it was really great I was joining something, getting out exer -I don't my family \& they were excited...but then asked how long is the program...I'm sure when Friday comes pat on the back

-my entire family does [supports] - my girls were just as happy as can be that we were both doing it [exercise] -my daughters...everyday...will say are you going to ex? Oh today is the last day. Now you'll need to continue support has been wonderful

-my husband too... as I go out the door he will say, you're looking good

-my husband...were very happy I was moving. And they were encouraging along the way. I tried to have them the water...

-I have a cousin who needed to ex and had mentioned that her doctor recommended aqua therapy so I suggested would sign up to encourage her

-we decided to do it together

-hear about it through my wife

-I got into it because my wife wanted to otherwise I wouldn't have done it, I'm glad I did

-my only disappointment this whole study was that my husband got in the control group. He is going to be enrol 
I'm going to sign up too. We'll do it together then

-I may be forced to take up walking. My husband walks $20 \mathrm{mi} / \mathrm{wk} \&$ he has been preaching to me that I need to basis

-I think most people need a buddy system. Whether it is someone at home or, prior to my husband retiring, my c did everything together. I really think you need support from somebody...somebody telling you to get your stuf go.

-my husband, he suffers from dementia some \& I think the reason he pushed me into this was...

-wanted support my cousin

-\& my daughter and my husband were very good...just keep going... keep doing it

\section{FRIEND:}

-I think most people need a buddy system. Whether it is someone at home or, prior to my husband retiring, my c did everything together. I really think you need support from somebody...somebody telling you to get your stuf go.

-commitment. If you are going to meet someone to walk...you follow through even though its hard some morni -I'd love to walk with somebody but all my friends are non-ambulatory

-I have a friend in the AM class. When she got wind of it she let me know

-we did a lot of sharing. Personal lives, that was wonderful. encourage, I think we encouraged one another...we -my friends, you know, 'just keep going'

-I wanted to come \& see the people. You all were a big draw for me. That's why I think a buddy system is such have a friend helping

-my friends were very happy I was moving and they were encouraging along the way. I tried to have them teach water because they were all coordinated.

\section{EXERCISE GROUP:}

-I've met a lot of nice people

-easier to be motivated when your with a group of people

-when I lost my husband....[strong group support]

-we motivate each other too...we don't want to let the other done down. So I think group exercise helps us

$-\&$ all the people you are with have a lot to do with it [fun]

-I had access... to HW... with the group

-[in relation to support] being part of group - GROUP AGREES

-group participation, trying to get self-motivated

-I love the people

-I wanted to see the people...you all were a big draw for me

-I haven't seen anything but support...from the other participants, the students

-a lot of nice group mates

-we accepted each other as individuals in the same manner. We made each other feel comfortable

-we were a very happy group. I'm sorry to see it end

-I'd like to than the ladies for making me feel comfortable [only guy in group]

\begin{tabular}{|c|c|c|c|c|c|}
\hline \multirow{2}{*}{ Self-Efficacy } & \multicolumn{4}{|c|}{ Quantitative Findings (n = 17) } \\
\cline { 2 - 5 } & \multicolumn{2}{|c|}{ Pre } & \multicolumn{2}{|c|}{ Post } & p-value \\
\cline { 2 - 6 } & 3.35 & $\mathrm{SD}=0.87$ & 3.29 & $\mathrm{SD}=0.67$ & $\mathrm{p}=0$. \\
\hline
\end{tabular}

\section{Qualitative Findings $(n=14)$}

\section{HEALTH AND ABILITY LEVEL}

-I joined it \& have really enjoyed it

-was exposed to water exercise and enjoyed it [previous experience]

-it does help you to do your activities and everything at 1 time... if you don't use it you lose it 
-feel better about myself that I'm doing something proactive

-when I first started I needed recuperation time...now my day is easier

-balanced increased...leaned to the left before

-my balance has gotten a lot better too... and my knees are better too

-my knees are better too...my knees have not been bothering me as much

-I have muscles for the first time in my life

-I have increased my daily activities doing this

-I can take stairs one step at a time...I couldn't do that at the beginning

-going from depending on a brace to not having to... big plus

-we went from raking leaves to shoveling snow, so I had to do a little of both. I found it a little easier to bend an -more energy... do more stuff...accomplish more

-I can get my foot up under my chin in water, but on land I can't even get up to my waist. So just being able to u easier way, a plus for me because if I get down on the floor, its really a chore to get back up...yes, I'm more mol -how you feel afterwards...it's kind of energizing

-I need the exercise [in reference to health concerns]

-it keeps me moving

-I see the difference, I can do things I couldn't

-I've lost weight, feel good about myself

-\& I do have arthritis starting and I've found that...I'm able to have more movement

-I don't have to wear my brace anymore

-I think it keeps you in shape, not only physically but mentally

-gives you more energy throughout the day \& it helps you move better and awful lot too

-it gives me energy

-I know exercise has always been good for my medical conditions

-I'm never going to make it up those steps...but I had no trouble...

-the exercise gives me more energy \& flexibility \& it also makes me feel better about myself knowing that I'm t: own body \& doing what is good for it.

-the energy is so much better now

-find that it [water exercise] is beneficial to me

-I do appreciate walking without pain [attributes to water exercise]

-it improves your mood too - GROUP AGREES

-I know exercise endorsed me very good emotionally. I was very depressed when I started and I'm much better $r$ -I've seen my parents \& my mom was too sore to move around and she doesn't move around, and her joints are think it's [exercise] a necessary part of life

-I walk alone, I'd love to walk with somebody but all my friends are non-ambulatory...but that does help me.

-I was struggling before and I was hoping it would help me

-it makes me feel better about myself that I'm doing something proactive

-it's scary because I'm afraid I'll lose it [ability to walk steps]

-my sister hyper-supports this... her husband has diabetes

-I haven't lost any weight and my husband is very disappointed in that*

-I struggled very much with the different steps*

-sometimes I felt like I was working really hard \& the HR monitor said I wasn't*

-sometimes the HR monitors were faulty* - LAUGHS

-struggled with coordination \& trying to do the steps*

FEAR OF WATER:

-can't swim a lick, but I love it \& after I got comfortable \& turned the wall lose... 
-that was the first time I had ever been in the water...definitely have fear of water...so some of the things, when the floor, you lose me

\section{KNOWLEDGE:}

-with my new knowledge of what exercise is from the pool that'll be more beneficial and then I'll have access to [joining a gym]

-liked the fact that class was structured...telling us what to do and constantly kept us moving...if I had to do it o would have periods of time when I do nothing

-have done water exercise and I found it to be quite fruitful and helpful

-went there for exercise [past]...made me feel an awful lot better

-the only thing I did active before this was walking the dogs \& those dogs have back problems sot the don't wall get out much on my own...I need something structured

-feel like I was helping to find out if water exercise would help cognitive function...helping Lori, helping in som AGE:

-and I'm the oldest in the group [stated with pride]

-all of us as aging \& kind of forget that you can't do a whole lot of things if you don't have the real resolve...

-but at my age, if I exercise too much I will end up in a chair

-I really feel the older you get, the more proud you should be \& if you are up and about, you should be proud of

ACCOMPLISHMENT:

-sense of accomplishment...now I can do anything

-steps no trouble

-muscles for the $1^{\text {st }}$ time in my life

-no I can take the stairs one step at time...I couldn't in the beginning

-more energy... do more stuff... accomplish more

-how good you feel afterwards...it is kind of energizing

-self-motivated. I like the bragging rights to tell my kids.

-I'm pretty much self-motivated.

-I'm really proud of myself 
Appendix M: Matched Pairs

\begin{tabular}{|c|c|c|c|c|c|c|c|c|c|}
\hline $\begin{array}{l}\text { Participant } \\
\#\end{array}$ & Age & Sex & $\begin{array}{l}\text { FITNESS } \\
\text { PARAMETERS }\end{array}$ & GROUP & $\begin{array}{l}\text { Participant } \\
\#\end{array}$ & Age & Sex & $\begin{array}{l}\text { FITNESS } \\
\text { PARAMETERS }\end{array}$ & GROUP \\
\hline 037 & 84 & $F$ & $\begin{array}{l}\text { RHR }-93 \\
\text { RBP }-136 / 70 \\
\text { BODY FAT - } \\
46.4 \%\end{array}$ & & 015 & 77 & $F$ & $\begin{array}{l}\text { RHR - 62 } \\
\text { RBP - 153/77 } \\
\text { BODY FAT - } \\
41.7\end{array}$ & \\
\hline 004 & 69 & $F$ & $\begin{array}{l}\text { RHR }-72 \\
\text { RBP - 112/68 } \\
\text { BODY FAT - } \\
57.1 \%\end{array}$ & & 022 & 67 & $F$ & $\begin{array}{l}\text { RHR - 76 } \\
\text { RBP - 126/62 } \\
\text { BODY FAT - } \\
51.8 \%\end{array}$ & \\
\hline 005 & 60 & $F$ & $\begin{array}{l}\text { RHR }-75 \\
\text { RBP }-117 / 61 \\
\text { BODY FAT - } \\
33.0\end{array}$ & & 010 & 61 & $F$ & $\begin{array}{l}\text { RHR - 71 } \\
\text { RBP - 112/63 } \\
\text { BODY FAT - } \\
33.7 \%\end{array}$ & \\
\hline 006 & 68 & $F$ & $\begin{array}{l}\text { RHR }-65 \\
\text { RBP }-128 / 64 \\
\text { BODY FAT - } \\
45.9 \%\end{array}$ & & 014 & 69 & $F$ & $\begin{array}{l}\text { RHR }-50 \\
\text { RBP - 145/65 } \\
\text { BODY FAT - } \\
43.9 \%\end{array}$ & \\
\hline 011 & 67 & $M$ & $\begin{array}{l}\text { RHR }-80 \\
\text { RBP }-138 / 87 \\
\text { BODY FAT - } \\
39.5 \%\end{array}$ & & 008 & 66 & $M$ & $\begin{array}{l}\text { RHR - 81 } \\
\text { RBP - 140/83 } \\
\text { BODY FAT - } \\
\text { measurement } \\
\text { needs to be } \\
\text { redone }\end{array}$ & \\
\hline 007 & 60 & $M$ & $\begin{array}{l}\text { RHR }-64 \\
\text { RBP - 146/77 } \\
\text { BODY FAT - } \\
38.3\end{array}$ & & 040 & 62 & $M$ & $\begin{array}{l}\text { RHR }-65 \\
\text { RBP - 159/78 } \\
\text { BODY FAT - } \\
37.5 \%\end{array}$ & \\
\hline 009 & 85 & $F$ & $\begin{array}{l}\text { RHR }-63 \\
\text { RBP - 154/75 } \\
\text { BODY FAT - } \\
45.3 \%\end{array}$ & & 017 & 83 & $F$ & $\begin{array}{l}\text { RHR }-89 \\
\text { RBP }-200 / 85 \\
\text { BODY FAT - } \\
43 \%\end{array}$ & \\
\hline 012 & 68 & $M$ & $\begin{array}{l}\text { RHR }-66 \\
\text { RBP }-140 / 82 \\
\text { BODY FAT - } \\
47.7\end{array}$ & & 029 & 63 & $M$ & $\begin{array}{l}\text { RHR }-59 \\
\text { RBP }-152 / 68 \\
\text { BODY FAT - } \\
32.6 \%\end{array}$ & \\
\hline 013 & 78 & $\mathrm{~F}$ & $\begin{array}{l}\text { RHR }-57 \\
\text { RBP }-133 / 58 \\
\text { BODY FAT - } \\
50.8 \%\end{array}$ & & 025 & 74 & $\mathrm{~F}$ & $\begin{array}{l}\text { RHR }-68 \\
\text { RBP }-144 / 72 \\
\text { BODY FAT - } \\
44.1 \%\end{array}$ & \\
\hline 018 & 63 & $F$ & $\begin{array}{l}\text { RHR }-61 \\
\text { RBP }-144 / 69 \\
\text { BODY FAT - } \\
44.3\end{array}$ & & 033 & 65 & $F$ & $\begin{array}{l}\text { RHR }-83 \\
\text { RBP }-122 / 62 \\
\text { BODY FAT - } \\
49.8 \%\end{array}$ & \\
\hline 019 & 60 & $\mathrm{~F}$ & RHR - 59 & & 036 & 60 & $\mathrm{~F}$ & $\mathrm{RHR}-72$ & \\
\hline
\end{tabular}




\begin{tabular}{|c|c|c|c|c|c|c|c|}
\hline & & & $\begin{array}{l}\text { RBP - 115/68 } \\
\text { BODY FAT - } \\
32.5 \%\end{array}$ & & & & $\begin{array}{l}\text { RBP - 98/62 } \\
\text { BODY FAT - } \\
33 \%\end{array}$ \\
\hline 020 & 64 & $F$ & $\begin{array}{l}\text { RHR }-63 \\
\text { RBP }-97 / 60 \\
\text { BODY FAT - } \\
40 \%\end{array}$ & 034 & 64 & $F$ & $\begin{array}{l}\text { RHR }-61 \\
\text { RBP - 126/78 } \\
\text { BODY FAT - } \\
38.3 \%\end{array}$ \\
\hline 021 & 61 & $M$ & $\begin{array}{l}\text { RHR }-61 \\
\text { RBP }-119 / 76 \\
\text { BODY FAT - } \\
30.4 \% \\
\end{array}$ & 031 & 60 & $M$ & $\begin{array}{l}\text { RHR }-97 \\
\text { RBP - 146/86 } \\
\text { BODY FAT - } \\
29.8 \% \\
\end{array}$ \\
\hline 023 & 79 & $\mathrm{M}$ & $\begin{array}{l}\text { RHR }-119 \\
\text { RBP }-107 / 58 \\
\text { BODY FAT - } \\
29.2 \% \\
\end{array}$ & 026 & 82 & $M$ & $\begin{array}{l}\text { RHR }-54 \\
\text { RBP }-113 / 52 \\
\text { BODY FAT - } \\
33.2 \% \\
\end{array}$ \\
\hline 027 & 68 & $F$ & $\begin{array}{l}\text { RHR }-65 \\
\text { RBP - 104/47 } \\
\text { BODY FAT - } \\
35.5 \% \\
\end{array}$ & 028 & 67 & $F$ & $\begin{array}{l}\text { RHR - 50 } \\
\text { RBP - 135/73 } \\
\text { BODY FAT - } \\
41.8 \% \\
\end{array}$ \\
\hline 030 & 64 & $F$ & $\begin{array}{l}\text { RHR }-61 \\
\text { RBP }-120 / 75 \\
\text { BODY FAT - } \\
31.2 \%\end{array}$ & 039 & 60 & $F$ & $\begin{array}{l}\text { RHR }-68 \\
\text { RBP - 119/59 } \\
\text { BODY FAT - } \\
52 \%\end{array}$ \\
\hline 035 & 62 & $F$ & $\begin{array}{l}\text { RHR }-90 \\
\text { RBP - 150/84 } \\
\text { BODY FAT - } \\
45.5 \% \\
\end{array}$ & 041 & 60 & $F$ & $\begin{array}{l}\text { RHR }-67 \\
\text { RBP - 150/87 } \\
\text { BODY FAT - } \\
46.4 \% \\
\end{array}$ \\
\hline 042 & 66 & $F$ & $\begin{array}{l}\text { RHR - 64 } \\
\text { RBP - 106/62 } \\
\text { BODY FAT - } \\
49.5 \%\end{array}$ & 043 & 66 & $F$ & $\begin{array}{l}\text { RHR }-74 \\
\text { RBP - 151/77 } \\
\text { BODY FAT - } \\
36.9 \%\end{array}$ \\
\hline 024 & 79 & $F$ & $\begin{array}{l}\text { RHR }-72 \\
\text { RBP }-137 / 63 \\
\text { BODY FAT - } \\
28.5 \%\end{array}$ & 002 & 75 & $F$ & $\begin{array}{l}\text { RHR }-52 \\
\text { RBP - 146/62 } \\
\text { BODY FAT - } \\
39.7 \%\end{array}$ \\
\hline 003 & 63 & $M$ & $\begin{array}{l}\text { RHR }-73 \\
\text { RBP }-107 / 60 \\
\text { BODY FAT - } \\
19.9 \%\end{array}$ & 032 & 68 & $F$ & $\begin{array}{l}\text { Parameters } \\
\text { collected on } \\
\text { Tuesday...was } \\
\text { out of town } \\
\text { for } 2^{\text {nd }} \\
\text { appointment } \\
\text { and will } \\
\text { double-up } \\
\text { this week } \\
\text { (Tuesday \& } \\
\text { Friday) }\end{array}$ \\
\hline
\end{tabular}




\section{Reference List}

Exercise and Type 2 Diabetes: American College of Sports Medicine and the American Diabetes Association: Joint Position Statement (2010). Medicine \& Science in Sports \& Exercise, 42.

Abbasi, A., Sadeghi, H., Tabrizi, H. B., Bagheri, K., \& Ghasemizad, A. (2012). Effects of aquatic balance training and detraining on neuromuscular performance and balance in healthy middle aged male. Koomesh, 13, e345-Pe353, En46.

Adams, J. A. (1971). A closed-loop theory of motor learning. J Mot.Behav., 3, 111-149.

Adlard, P. A., Engesser-Cesar, C., \& Cotman, C. W. (2011). Mild stress facilitates learning and exercise improves retention in aged mice. Exp.Gerontol., 46, 53-59.

Administration on Aging (2011). Department of Health \& Human Services. Retrieved 328-2013, from http://www.aoa.gov/Aging_Statistics/

Ahamed, Y., Macdonald, H., Reed, K., Naylor, P. J., Liu-Ambrose, T., \& McKay, H. (2007). School-based physical activity does not compromise children's academic performance. Med.Sci.Sports Exerc., 39, 371-376.

Ainsworth, B. E., Haskell, W. L., Leon, A. S., Jacobs, D. R., Jr., Montoye, H. J., Sallis, J. F. et al. (1993). Compendium of physical activities: classification of energy costs of human physical activities. Med.Sci.Sports Exerc., 25, 71-80. 
Alkarmi, A., Thijssen, D. H., Albouaini, K., Cable, N. T., Wright, D. J., Green, D. J. et al. (2010). Arterial prehabilitation: can exercise induce changes in artery size and function that decrease complications of catheterization? Sports Med, 40, 481-492.

Alzheimer's Association (2011). Alzheimer's Disease Facts and Figures. Alzhemier's \& Dementia, 7.

Ambroza, C. \& Geigle, P. R. (2010). Aquatic Exercise as a Management Tool for Breast Cancer-Related Lymphedema. Topics in Geriatric Rehabilitation, 26.

American Heart Association (2012). Cardiovascular Disease and Diabetes.

Anderton, B. H. (2002). Ageing of the brain. Mech.Ageing Dev., 123, 811-817.

Angevaren, M., Aufdemkampe, G., Verhaar, H. J., Aleman, A., \& Vanhees, L. (2008). Physical activity and enhanced fitness to improve cognitive function in older people without known cognitive impairment. Cochrane.Database.Syst.Rev., CD005381.

Angevaren, M., Vanhees, L., Nooyens, A. C., Wendel-Vos, C. G., \& Verschuren, W. M. (2010). Physical activity and 5-year cognitive decline in the Doetinchem cohort study. Ann.Epidemiol., 20, 473-479.

Anguera, J. A., Reuter-Lorenz, P. A., Willingham, D. T., \& Seidler, R. D. (2010). Contributions of spatial working memory to visuomotor learning. J Cogn Neurosci., 22, 19171930. 
Anguera, J. A., Reuter-Lorenz, P. A., Willingham, D. T., \& Seidler, R. D. (2011). Failure to engage spatial working memory contributes to age-related declines in visuomotor learning. $J$ Cogn Neurosci., 23, 11-25.

Asl, N. A., Sheikhzade, F., Torchi, M., Roshangar, L., \& Khamnei, S. (2008). Long-term regular exercise promotes memory and learning in young but not in older rats. Pathophysiology., $15,9-12$.

Atalay M \& DE Laaksonen (2002). Diebetes,Oxidative Stress \& Physical Exercise. Journal of Sports Science and Medicine, 1, 1-14.

Badcock, J. C., Michiel, P. T., \& Rock, D. (2005). Spatial working memory and planning ability: contrasts between schizophrenia and bipolar I disorder. Cortex, 41, 753-763.

Baker, L. D., Frank, L. L., Foster-Schubert, K., Green, P. S., Wilkinson, C. W., McTiernan, A. et al. (2010). Aerobic exercise improves cognition for older adults with glucose intolerance, a risk factor for Alzheimer's disease. J.Alzheimers.Dis., 22, 569-579.

Balde, A., Figueras, J., Hawking, D., \& Miller, J. (2003). Physician advice to the elderly about physical acvitity. Journal of Aging \& Physical Activity, 11, 90-97.

Balldin, U. I., Dahlback, G. O., \& Lundgren, C. E. (1971). Changes in vital capacity produced by oxygen breathing during immersion with the head above water. Aerosp.Med., 42, 384-387. 
Baltes, P. B. \& Lindenberger, U. (1997). Emergence of a powerful connection between sensory and cognitive functions across the adult life span: a new window to the study of cognitive aging? Psychol.Aging, 12, 12-21.

Bandura, A. (2001). Social cognitive theory: an agentic perspective. Annu.Rev.Psychol., $52,1-26$.

Bandura, A. (2004). Health promotion by social cognitive means. Health Educ.Behav., $31,143-164$.

Barnes, D. E., Cauley, J. A., Lui, L. Y., Fink, H. A., McCulloch, C., Stone, K. L. et al. (2007). Women who maintain optimal cognitive function into old age. J.Am.Geriatr.Soc., 55, 259-264.

Barnes, D. E., Santos-Modesitt, W., Poelke, G., Kramer, A. F., Castro, C., Middleton, L. E. et al. (2013). The Mental Activity and eXercise (MAX) trial: a randomized controlled trial to enhance cognitive function in older adults. JAMA Intern.Med, 173, 797-804.

Barnes, D. E. \& Yaffe, K. (2011). The projected effect of risk factor reduction on Alzheimer's disease prevalence. Lancet Neurol., 10, 819-828.

Barnes, D. E., Yaffe, K., Satariano, W. A., \& Tager, I. B. (2003). A longitudinal study of cardiorespiratory fitness and cognitive function in healthy older adults. J.Am.Geriatr.Soc., 51, 459-465. 
Barnes, P. M. \& Schoenborn, C. A. (2012). Trends in adults receiving a recommendation for exercise or other physical activity from a physician or other health professional. NCHS.Data Brief., 1-8.

Barnett, I., van Sluijs, E. M., \& Ogilvie, D. (2012). Physical activity and transitioning to retirement: a systematic review. Am.J Prev.Med, 43, 329-336.

Barnett, I., van, S. E., Ogilvie, D., \& Wareham, N. J. (2014). Changes in household, transport and recreational physical activity and television viewing time across the transition to retirement: longitudinal evidence from the EPIC-Norfolk cohort. J Epidemiol.Community Health.

Barnett, J. H., Robbins, T. W., Leeson, V. C., Sahakian, B. J., Joyce, E. M., \& Blackwell, A. D. (2010). Assessing cognitive function in clinical trials of schizophrenia. Neurosci.Biobehav.Rev., 34, 1161-1177.

Bartzokis, G., Cummings, J. L., Sultzer, D., Henderson, V. W., Nuechterlein, K. H., \& Mintz, J. (2003). White matter structural integrity in healthy aging adults and patients with Alzheimer disease: a magnetic resonance imaging study. Arch.Neurol., 60, 393-398.

Basile, J. N. (2002). Systolic blood pressure. BMJ, 325, 917-918.

Bates, K. A., Sohrabi, H. R., Rodrigues, M., Beilby, J., Dhaliwal, S. S., Taddei, K. et al. (2009a). Association of cardiovascular factors and Alzheimer's disease plasma amyloid-beta protein in subjective memory complainers. J.Alzheimers.Dis., 17, 305-318. 
Bates, K. A., Verdile, G., Li, Q. X., Ames, D., Hudson, P., Masters, C. L. et al. (2009b). Clearance mechanisms of Alzheimer's amyloid-beta peptide: implications for therapeutic design and diagnostic tests. Mol.Psychiatry, 14, 469-486.

Bauman, A. E., Reis, R. S., Sallis, J. F., Wells, J. C., Loos, R. J., \& Martin, B. W. (2012). Correlates of physical activity: why are some people physically active and others not? Lancet, 380, 258-271.

Bauman, A. E., Sallis, J. F., Dzewaltowski, D. A., \& Owen, N. (2002). Toward a better understanding of the influences on physical activity: the role of determinants, correlates, causal variables, mediators, moderators, and confounders. Am.J.Prev.Med., 23, 5-14.

Baumgartner, R. N., Wayne, S. J., Waters, D. L., Janssen, I., Gallagher, D., \& Morley, J. E. (2004). Sarcopenic obesity predicts instrumental activities of daily living disability in the elderly. Obes.Res., 12, 1995-2004.

Becker BE \& AJ Cole (2011). Comprehensive Aquatic Therapy. (3rd ed.) Pullman WA: Washington State University Publishing.

Becker, B. E. (2009). Aquatic therapy: scientific foundations and clinical rehabilitation applications. PM.R., 1, 859-872.

Belza, B., Topolski, T., Kinne, S., Patrick, D. L., \& Ramsey, S. D. (2002). Does adherence make a difference? Results from a community-based aquatic exercise program. Nurs.Res., 51, 285-291. 
Benloucif, S., Orbeta, L., Ortiz, R., Janssen, I., Finkel, S. I., Bleiberg, J. et al. (2004). Morning or evening activity improves neuropsychological performance and subjective sleep quality in older adults. Sleep, 27, 1542-1551.

Bennell, K. L. \& Hinman, R. S. (2011). A review of the clinical evidence for exercise in osteoarthritis of the hip and knee. J.Sci.Med.Sport, 14, 4-9.

Berkman, L. F., Seeman, T. E., Albert, M., Blazer, D., Kahn, R., Mohs, R. et al. (1993). High, usual and impaired functioning in community-dwelling older men and women: findings from the MacArthur Foundation Research Network on Successful Aging. J.Clin.Epidemiol., 46, 1129-1140.

Bielak, A. A. (2010). How can we not 'lose it' if we still don't understand how to 'use it'? Unanswered questions about the influence of activity participation on cognitive performance in older age--a mini-review. Gerontology, 56, 507-519.

Binder, E. F., Storandt, M., \& Birge, S. J. (1999). The relation between psychometric test performance and physical performance in older adults. J.Gerontol.A Biol.Sci.Med.Sci., 54, M428-M432.

Birren, J. E. \& Fisher, L. M. (1995). Aging and speed of behavior: possible consequences for psychological functioning. Annu.Rev.Psychol., 46, 329-353.

Black, J. E., Isaacs, K. R., Anderson, B. J., Alcantara, A. A., \& Greenough, W. T. (1990). Learning causes synaptogenesis, whereas motor activity causes angiogenesis, in cerebellar cortex of adult rats. Proc.Natl.Acad.Sci.U.S.A, 87, 5568-5572. 
Blackmore, D. G., Golmohammadi, M. G., Large, B., Waters, M. J., \& Rietze, R. L. (2009). Exercise increases neural stem cell number in a growth hormone-dependent manner, augmenting the regenerative response in aged mice. Stem Cells, 27, 2044-2052.

Blumenthal, J. A. \& Madden, D. J. (1988). Effects of aerobic exercise training, age, and physical fitness on memory-search performance. Psychol.Aging, 3, 280-285.

Booth, M. L., Bauman, A., Owen, N., \& Gore, C. J. (1997). Physical activity preferences, preferred sources of assistance, and perceived barriers to increased activity among physically inactive Australians. Prev.Med., 26, 131-137.

Booth, M. L., Owen, N., Bauman, A., Clavisi, O., \& Leslie, E. (2000). Social-cognitive and perceived environment influences associated with physical activity in older Australians. Prev.Med., 31, 15-22.

Brach, J. S., Van Swearingen, J. M., Perera, S., Wert, D. M., \& Studenski, S. (2013). Motor learning versus standard walking exercise in older adults with subclinical gait dysfunction: a randomized clinical trial. J Am.Geriatr.Soc., 61, 1879-1886.

Brassington, G. S., Atienza, A. A., Perczek, R. E., DiLorenzo, T. M., \& King, A. C. (2002). Intervention-related cognitive versus social mediators of exercise adherence in the elderly. Am.J.Prev.Med., 23, 80-86.

Brown, L. A., Shumway-Cook, A., \& Woollacott, M. H. (1999). Attentional demands and postural recovery: the effects of aging. J Gerontol.A Biol.Sci.Med Sci., 54, M165-M171. 
Buckner, R. L. (2004). Memory and executive function in aging and AD: multiple factors that cause decline and reserve factors that compensate. Neuron, 44, 195-208.

Buckworth, J. \& Dishman, R. K. (2002). Exercise psychology. Human kinetics.

Budde, H., Voelcker-Rehage, C., Pietrabyk-Kendziorra, S., Ribeiro, P., \& Tidow, G. (2008). Acute coordinative exercise improves attentional performance in adolescents. Neurosci.Lett., 441, 219-223.

Burns, J. M., Cronk, B. B., Anderson, H. S., Donnelly, J. E., Thomas, G. P., Harsha, A. et al. (2008). Cardiorespiratory fitness and brain atrophy in early Alzheimer disease. Neurology, 71, 210-216.

Burton, L. C., Shapiro, S., \& German, P. S. (1999). Determinants of physical activity initiation and maintenance among community-dwelling older persons. Prev.Med., 29, 422-430.

Cabeza, R., Anderson, N. D., Locantore, J. K., \& McIntosh, A. R. (2002). Aging gracefully: compensatory brain activity in high-performing older adults. Neuroimage., 17, 13941402.

Cadore, E. L., Rodriguez-Manas, L., Sinclair, A., \& Izquierdo, M. (2013). Effects of different exercise interventions on risk of falls, gait ability, and balance in physically frail older adults: a systematic review. Rejuvenation.Res., 16, 105-114.

Carles S Jr, Curnier, D., Pathak, A., Roncalli, J., Bousquet, M., Garcia, J. L. et al. (2007). Effects of short-term exercise and exercise training on cognitive function among patients with cardiac disease. J.Cardiopulm.Rehabil.Prev., 27, 395-399. 
Carro, E., Trejo, J. L., Busiguina, S., \& Torres-Aleman, I. (2001). Circulating insulin-like growth factor I mediates the protective effects of physical exercise against brain insults of different etiology and anatomy. J.Neurosci., 21, 5678-5684.

CDC (2011). Implications of an Aging Society. http://www.cdc.gov/chronicdisease/resources/publications/AAG/aging.htm. Retrieved 3-282013, from http://www.cdc.gov/chronicdisease/resources/publications/AAG/aging.htm

CDC (2014). Ambulatory Care Use and Physician Visits. http://www.cdc.gov/nchs/fastats/docvisit.htm

Center for Disease Control (2012). Dementia/Alzheimer's Disease.

Centers for Disease Control and Prevention (2012). Heart Disease. cdc.gov. cdc.gov/heartdisease/facts.htm

Chaddock, L., Erickson, K. I., Prakash, R. S., Kim, J. S., Voss, M. W., VanPatter, M. et al. (2010). A neuroimaging investigation of the association between aerobic fitness, hippocampal volume, and memory performance in preadolescent children. Brain Res., 1358, 172-183.

Chaddock, L., Hillman, C. H., Buck, S. M., \& Cohen, N. J. (2011). Aerobic fitness and executive control of relational memory in preadolescent children. Med.Sci.Sports Exerc., 43, 344-349.

Chan, A. S., Ho, Y. C., Cheung, M. C., Albert, M. S., Chiu, H. F., \& Lam, L. C. (2005). Association between mind-body and cardiovascular exercises and memory in older adults. J.Am.Geriatr.Soc., 53, 1754-1760. 
Chan, R. C., Shum, D., Toulopoulou, T., \& Chen, E. Y. (2008). Assessment of executive functions: review of instruments and identification of critical issues. Arch.Clin.Neuropsychol., 23, 201-216.

Chiviacowsky, S., Campos, T., \& Domingues, M. R. (2010). Reduced frequency of knowledge of results enhances learning in persons with Parkinson's disease. Front Psychol., 1, 226.

Chiviacowsky, S. \& Wulf, G. (2007). Feedback after good trials enhances learning. Res.Q.Exerc.Sport, 78, 40-47.

Chiviacowsky, S., Wulf, G., \& Wally, R. (2010). An external focus of attention enhances balance learning in older adults. Gait.Posture., 32, 572-575.

Chodzko-Zajko \& DN Proctor, M. F. S. C. M. C. N. G. S. J. S. (2009). Exercise and Physical Activity for Older Adults. Medicine \& Science in Sports \& Exercise, 41, 1510-1530.

Chuang, Y. F., Eldreth, D., Erickson, K. I., Varma, V., Harris, G., Fried, L. P. et al. (2013). Cardiovascular risks and brain function: a functional magnetic resonance imaging study of executive function in older adults. Neurobiol.Aging.

Chun, M. M. \& Turk-Browne, N. B. (2007). Interactions between attention and memory. Curr.Opin.Neurobiol., 17, 177-184.

Chung, S., Domino, M. E., Stearns, S. C., \& Popkin, B. M. (2009). Retirement and physical activity: analyses by occupation and wealth. Am.J Prev.Med, 36, 422-428. 
Clarkson-Smith, L. \& Hartley, A. A. (1989). Relationships between physical exercise and cognitive abilities in older adults. Psychol.Aging, 4, 183-189.

Clarkson-Smith, L. \& Hartley, A. A. (1990). Structural equation models of relationships between exercise and cognitive abilities. Psychol.Aging, 5, 437-446.

Coe, D. P., Pivarnik, J. M., Womack, C. J., Reeves, M. J., \& Malina, R. M. (2006). Effect of physical education and activity levels on academic achievement in children. Med.Sci.Sports Exerc., 38, 1515-1519.

Cohen-Mansfield, J. (2003). Nonpharmacologic interventions for psychotic symptoms in dementia. J.Geriatr.Psychiatry Neurol., 16, 219-224.

Cohen-Mansfield, J., Marx, M. S., \& Guralnik, J. M. (2006). Comparison of exercise models in an elderly population. Aging Clin.Exp.Res., 18, 312-319.

Colado, J. C., Tella, V., \& Triplett, N. T. (2008). A method for monitoring intensity during aquatic resistance exercises. J.Strength.Cond.Res., 22, 2045-2049.

Colado, J. C., Tella, V., Triplett, N. T., \& Gonzalez, L. M. (2009). Effects of a short-term aquatic resistance program on strength and body composition in fit young men. J.Strength.Cond.Res., 23, 549-559.

Colado, J. C., Triplett, N. T., Tella, V., Saucedo, P., \& Abellan, J. (2009). Effects of aquatic resistance training on health and fitness in postmenopausal women. Eur.J.Appl.Physiol, $106,113-122$. 
Colcombe, S. \& Kramer, A. F. (2003). Fitness effects on the cognitive function of older adults: a meta-analytic study. Psychol.Sci., 14, 125-130.

Coles, K. \& Tomporowski, P. D. (2008). Effects of acute exercise on executive processing, short-term and long-term memory. J.Sports Sci., 26, 333-344.

Collins JL (1995). Understanding and Promoting Physical Activity CDC.

Cruise, K. E., Bucks, R. S., Loftus, A. M., Newton, R. U., Pegoraro, R., \& Thomas, M. G. (2011). Exercise and Parkinson's: benefits for cognition and quality of life. Acta Neurol.Scand., 123, 13-19.

Davis, C. L., Tomporowski, P. D., Boyle, C. A., Waller, J. L., Miller, P. H., Naglieri, J. A. et al. (2007). Effects of aerobic exercise on overweight children's cognitive functioning: a randomized controlled trial. Res.Q.Exerc.Sport, 78, 510-519.

Davis, S. W., Dennis, N. A., Daselaar, S. M., Fleck, M. S., \& Cabeza, R. (2008). Que PASA? The posterior-anterior shift in aging. Cereb.Cortex, 18, 1201-1209.

Deeny, S. P., Poeppel, D., Zimmerman, J. B., Roth, S. M., Brandauer, J., Witkowski, S. et al. (2008). Exercise, APOE, and working memory: MEG and behavioral evidence for benefit of exercise in epsilon4 carriers. Biol.Psychol., 78, 179-187.

Denkinger, M. D., Nikolaus, T., Denkinger, C., \& Lukas, A. (2012). Physical activity for the prevention of cognitive decline: current evidence from observational and controlled studies. Z.Gerontol.Geriatr., 45, 11-16. 
Dik, M., Deeg, D. J., Visser, M., \& Jonker, C. (2003). Early life physical activity and cognition at old age. J.Clin.Exp.Neuropsychol., 25, 643-653.

DiLorenzo, T. M., Bargman, E. P., Stucky-Ropp, R., Brassington, G. S., Frensch, P. A., \& LaFontaine, T. (1999). Long-term effects of aerobic exercise on psychological outcomes. Prev.Med., 28, 75-85.

Ding, Q., Vaynman, S., Akhavan, M., Ying, Z., \& Gomez-Pinilla, F. (2006). Insulin-like growth factor I interfaces with brain-derived neurotrophic factor-mediated synaptic plasticity to modulate aspects of exercise-induced cognitive function. Neuroscience, 140, 823-833.

Dishman, R. K. \& Ickes, W. (1981). Self-motivation and adherence to therapeutic exercise. J.Behav.Med., 4, 421-438.

Dogra, S. \& Stathokostas, L. (2012). Sedentary behavior and physical activity are independent predictors of successful aging in middle-aged and older adults. J.Aging Res., 2012, 190654.

Egerhazi, A., Berecz, R., Bartok, E., \& Degrell, I. (2007). Automated Neuropsychological Test Battery (CANTAB) in mild cognitive impairment and in Alzheimer's disease. Prog.Neuropsychopharmacol.Biol.Psychiatry, 31, 746-751.

Elliott, E. M., Cherry, K. E., Brown, J. S., Smitherman, E. A., Jazwinski, S. M., Yu, Q. et al. (2011). Working memory in the oldest-old: evidence from output serial position curves. Mem.Cognit., 39, 1423-1434.

Enright, P. L. (2003). The six-minute walk test. Respir.Care, 48, 783-785. 
Erickson, K. I., Prakash, R. S., Voss, M. W., Chaddock, L., Hu, L., Morris, K. S. et al. (2009). Aerobic fitness is associated with hippocampal volume in elderly humans. Hippocampus, 19, 1030-1039.

Erickson, K. I., Voss, M. W., Prakash, R. S., Basak, C., Szabo, A., Chaddock, L. et al. (2011). Exercise training increases size of hippocampus and improves memory. Proc.Natl.Acad.Sci.U.S.A, 108, 3017-3022.

Etnier, J. L., Nowell, P. M., Landers, D. M., \& Sibley, B. A. (2006). A meta-regression to examine the relationship between aerobic fitness and cognitive performance. Brain Research Reviews, 52, 119-130.

Ettinger, W. H., Jr., Burns, R., Messier, S. P., Applegate, W., Rejeski, W. J., Morgan, T. et al. (1997). A randomized trial comparing aerobic exercise and resistance exercise with a health education program in older adults with knee osteoarthritis. The Fitness Arthritis and Seniors Trial (FAST). JAMA, 277, 25-31.

Evcik, D., Yigit, I., Pusak, H., \& Kavuncu, V. (2008). Effectiveness of aquatic therapy in the treatment of fibromyalgia syndrome: a randomized controlled open study. Rheumatol.Int., 28, 885-890.

Evenson, K. R., Buchner, D. M., \& Morland, K. B. (2012). Objective measurement of physical activity and sedentary behavior among US adults aged 60 years or older. Prev.Chronic.Dis., 9, E26. 
Fabre, C., Chamari, K., Mucci, P., Masse-Biron, J., \& Prefaut, C. (2002). Improvement of cognitive function by mental and/or individualized aerobic training in healthy elderly subjects. Int.J.Sports Med., 23, 415-421.

Farrell, P. M. J. V. C. (2011). ACSM's Advanced Exercise Physiology. (2nd ed.) Lippincott Williams \& Wilkins.

Fitts, P. M. (1992). The information capacity of the human motor system in controlling the amplitude of movement. 1954. J Exp.Psychol.Gen., 121, 262-269.

Floel, A., Ruscheweyh, R., Kruger, K., Willemer, C., Winter, B., Volker, K. et al. (2010). Physical activity and memory functions: are neurotrophins and cerebral gray matter volume the missing link? Neuroimage., 49, 2756-2763.

Foottit, J. \& Anderson, D. (2012). Associations between perception of wellness and health-related quality of life, comorbidities, modifiable lifestyle factors and demographics in older Australians. Australas.J Ageing, 31, 22-27.

Forestier, R., Desfour, H., Tessier, J. M., Francon, A., Foote, A. M., Genty, C. et al. (2010). Spa therapy in the treatment of knee osteoarthritis: a large randomised multicentre trial. Ann.Rheum.Dis., 69, 660-665.

Fowler, K. S., Saling, M. M., Conway, E. L., Semple, J. M., \& Louis, W. J. (2002). Paired associate performance in the early detection of DAT. J.Int.Neuropsychol.Soc., 8, 58-71. 
Friedenreich, C. M., Courneya, K. S., Neilson, H. K., Matthews, C. E., Willis, G., Irwin, M. et al. (2006). Reliability and validity of the Past Year Total Physical Activity Questionnaire. Am.J.Epidemiol., 163, 959-970.

Friedland, R. P., Fritsch, T., Smyth, K. A., Koss, E., Lerner, A. J., Chen, C. H. et al. (2001). Patients with Alzheimer's disease have reduced activities in midlife compared with healthy control-group members. Proc.Natl.Acad.Sci.U.S.A, 98, 3440-3445.

Fylkesnes, K. \& Forde, O. H. (1991). The Tromso Study: predictors of self-evaluated health--has society adopted the expanded health concept? Soc.Sci.Med, 32, 141-146.

Fylkesnes, K. \& Forde, O. H. (1992). Determinants and dimensions involved in selfevaluation of health. Soc.Sci.Med, 35, 271-279.

Galvan, V. \& Bredesen, D. E. (2007). Neurogenesis in the adult brain: implications for Alzheimer's disease. CNS.Neurol.Disord.Drug Targets., 6, 303-310.

Galvan, V. \& Jin, K. (2007). Neurogenesis in the aging brain. Clin.Interv.Aging, 2, 605610.

Gau, S. S. \& Shang, C. Y. (2010). Executive functions as endophenotypes in ADHD: evidence from the Cambridge Neuropsychological Test Battery (CANTAB). J Child Psychol.Psychiatry, 51, 838-849.

Gay, L. R., G.E.Mills, \& P.Airasian (2006). Educational Research: Competencies for Analysis and Application. (8th ed.) Berkley: Pearson Education Ltd. 
Geda, Y. E., Roberts, R. O., Knopman, D. S., Christianson, T. J., Pankratz, V. S., Ivnik, R. J. et al. (2010). Physical exercise, aging, and mild cognitive impairment: a population-based study. Arch.Neurol., 67, 80-86.

Geelen, R. J. \& Soons, P. H. (1996). Rehabilitation: an 'everyday' motivation model. Patient.Educ.Couns., 28, 69-77.

Glasgow, R. E., Eakin, E. G., Fisher, E. B., Bacak, S. J., \& Brownson, R. C. (2001). Physician advice and support for physical activity: results from a national survey. Am.J Prev.Med, 21, 189-196.

Goekint, M., Roelands, B., De, P. K., Knaepen, K., Bos, I., \& Meeusen, R. (2010). Does a period of detraining cause a decrease in serum brain-derived neurotrophic factor? Neurosci.Lett., 486, 146-149.

Goh, J. O., Beason-Held, L. L., An, Y., Kraut, M. A., \& Resnick, S. M. (2013). Frontal function and executive processing in older adults: process and region specific age-related longitudinal functional changes. Neuroimage., 69, 43-50.

Goldstein, D. J. (1992). Beneficial health effects of modest weight loss. Int.J Obes.Relat Metab Disord., 16, 397-415.

Goldstein, F. C., Ashley, A. V., Endeshaw, Y. W., Hanfelt, J., Lah, J. J., \& Levey, A. I. (2008). Effects of hypertension and hypercholesterolemia on cognitive functioning in patients with alzheimer disease. Alzheimer Dis.Assoc.Disord., 22, 336-342. 
Graef, F. I. s. \& Kruel, L. F. M. (2006). Freq+++ᄀncia card + ¡aca e percep $+{ }^{\circ}+$ úo subjetiva do esfor $+^{\circ}$ o no meio aqu +1 tico: diferen $+{ }^{\circ}$ as em rela $+^{\circ}+$ úo ao meio terrestre e aplica $+^{\circ}+{ }^{\prime}$ es na prescri $+{ }^{\circ}+$ úo do exerc+¡cio - uma revis+úo. Revista Brasileira de Medicina do Esporte, 12, 221228.

Green, D. J., Maiorana, A., O'Driscoll, G., \& Taylor, R. (2004). Effect of exercise training on endothelium-derived nitric oxide function in humans. J.Physiol, 561, 1-25.

Gulick, D. T. (2010). Effects of Aquatic Intervention on the Cardiopulmonary System in the Geriatric Population. Topics in Geriatric Rehabilitation, 26.

Gunther V, E. M. U. K. C. M. (1994). Fibromyalgia - the effect of relazation and hydrogavanic bath therapy on the subjective pain experience. Clinical Rheumatology, 13, 573578.

Guyton AC, J. H. (2011). Medical Physiology. (12th ed.) Philadelphia: W.B. Saunders Co.

Hale, L. A., Waters, D., \& Herbison, P. (2012). A randomized controlled trial to investigate the effects of water-based exercise to improve falls risk and physical function in older adults with lower-extremity osteoarthritis. Arch.Phys.Med.Rehabil., 93, 27-34.

Haskell, W. L., Lee, I. M., Pate, R. R., Powell, K. E., Blair, S. N., Franklin, B. A. et al. (2007). Physical activity and public health: updated recommendation for adults from the American College of Sports Medicine and the American Heart Association. Med Sci.Sports Exerc., 39, 1423-1434. 
Head, D., Buckner, R. L., Shimony, J. S., Williams, L. E., Akbudak, E., Conturo, T. E. et al. (2004). Differential vulnerability of anterior white matter in nondemented aging with minimal acceleration in dementia of the Alzheimer type: evidence from diffusion tensor imaging. Cereb.Cortex, 14, 410-423.

Hebert LE, S. P. B. J. B. D. E. D. (2003). Alzheimer disease in the us population: Prevalence estimates using the 2000 census. Archives of Neurology, 60, 1119-1122.

Hehman, J. A. \& Bugental, D. B. (2013). "Life stage-specific" variations in performance in response to age stereotypes. Dev.Psychol., 49, 1396-1406.

Herholz, K. (2003). PET studies in dementia. Ann.Nucl.Med., 17, 79-89.

Heyn, P., Abreu, B. C., \& Ottenbacher, K. J. (2004). The effects of exercise training on elderly persons with cognitive impairment and dementia: a meta-analysis. Arch.Phys.Med.Rehabil., 85, 1694-1704.

Heyn, P. C., Johnson, K. E., \& Kramer, A. F. (2008). Endurance and strength training outcomes on cognitively impaired and cognitively intact older adults: a meta-analysis. J.Nutr.Health Aging, 12, 401-409.

Hillman, C. H., Erickson, K. I., \& Kramer, A. F. (2008). Be smart, exercise your heart: exercise effects on brain and cognition. Nat.Rev.Neurosci., 9, 58-65.

Hillman, C. H., Pontifex, M. B., Raine, L. B., Castelli, D. M., Hall, E. E., \& Kramer, A. F. (2009). The effect of acute treadmill walking on cognitive control and academic achievement in preadolescent children. Neuroscience, 159, 1044-1054. 
Hillman, C. H., Snook, E. M., \& Jerome, G. J. (2003). Acute cardiovascular exercise and executive control function. Int.J.Psychophysiol., 48, 307-314.

Hogan, C. L., Mata, J., \& Carstensen, L. L. (2013). Exercise holds immediate benefits for affect and cognition in younger and older adults. Psychol.Aging, 28, 587-594.

Honda, T. \& Kamioka, H. (2012). Curative and health enhancement effects of aquatic exercise: evidence based on interventional studies. Open.Access.J Sports Med, 3, 27-34.

Hong, S. K., Cerretelli, P., Cruz, J. C., \& Rahn, H. (1969). Mechanics of respiration during submersion in water. J.Appl.Physiol, 27, 535-538.

Hultsch, D. F., Hertzog, C., Small, B. J., \& Dixon, R. A. (1999). Use it or lose it: engaged lifestyle as a buffer of cognitive decline in aging? Psychol.Aging, 14, 245-263.

Idler, E., Leventhal, H., McLaughlin, J., \& Leventhal, E. (2004). In sickness but not in health: self-ratings, identity, and mortality. J Health Soc.Behav., 45, 336-356.

Idler, E. L. \& Benyamini, Y. (1997). Self-rated health and mortality: a review of twentyseven community studies. J Health Soc.Behav., 38, 21-37.

Isaacs, K. R., Anderson, B. J., Alcantara, A. A., Black, J. E., \& Greenough, W. T. (1992). Exercise and the brain: angiogenesis in the adult rat cerebellum after vigorous physical activity and motor skill learning. J.Cereb.Blood Flow Metab, 12, 110-119.

Israel BA \& Schurman SJ (1990). Social support, control, and the stress process. In Glanz K, Lewis FM, \& Rimer BK (Eds.), Health behavior and health education: theory, research, and practice (pp. 187-215). San Fancisco: Jossey-Bass Publishers. 
Jack, S., West, M., \& Grocott, M. P. (2011). Perioperative exercise training in elderly subjects. Best.Pract.Res.Clin.Anaesthesiol., 25, 461-472.

Jacobs, H. I., Leritz, E. C., Williams, V. J., Van Boxtel, M. P., van der Elst, W., Jolles, J. et al. (2013). Association between white matter microstructure, executive functions, and processing speed in older adults: the impact of vascular health. Hum.Brain Mapp., 34, 77-95.

Janus, C., Pearson, J., McLaurin, J., Mathews, P. M., Jiang, Y., Schmidt, S. D. et al. (2000). A beta peptide immunization reduces behavioural impairment and plaques in a model of Alzheimer's disease. Nature, 408, 979-982.

Jazbec, S., Pantelis, C., Robbins, T., Weickert, T., Weinberger, D. R., \& Goldberg, T. E. (2007). Intra-dimensional/extra-dimensional set-shifting performance in schizophrenia: impact of distractors. Schizophr.Res., 89, 339-349.

Jensen, M. T., Suadicani, P., Hein, H. O., \& Gyntelberg, F. (2013). Elevated resting heart rate, physical fitness and all-cause mortality: a 16-year follow-up in the Copenhagen Male Study. Heart, 99, 882-887.

Jette, A. M., Lachman, M., Giorgetti, M. M., Assmann, S. F., Harris, B. A., Levenson, C. et al. (1999). Exercise--it's never too late: the strong-for-life program. Am.J Public Health, 89, $66-72$.

Joyce, E., Blumenthal, S., \& Wessely, S. (1996). Memory, attention, and executive function in chronic fatigue syndrome. J Neurol.Neurosurg.Psychiatry, 60, 495-503. 
Joyce, J., Smyth, P. J., Donnelly, A. E., \& Davranche, K. (2014). The simon task and aging: does acute moderate exercise influence cognitive control? Med Sci.Sports Exerc., 46, 630639.

Junkkila, J., Oja, S., Laine, M., \& Karrasch, M. (2012). Applicability of the CANTABPAL computerized memory test in identifying amnestic mild cognitive impairment and Alzheimer's disease. Dement.Geriatr.Cogn Disord., 34, 83-89.

Kamioka, H., Tsutani, K., Mutoh, Y., Okuizum, H., Ohta, M., Handa, S. et al. (2011). A systematic review of nonrandomized controlled trials on the curative effects of aquatic exercise. Int.J Gen.Med, 4, 239-260.

Kamioka, H., Tsutani, K., Okuizumi, H., Mutoh, Y., Ohta, M., Handa, S. et al. (2010). Effectiveness of aquatic exercise and balneotherapy: a summary of systematic reviews based on randomized controlled trials of water immersion therapies. J Epidemiol., 20, 2-12.

Kang, H. S., Ferrans, C. E., Kim, M. J., Kim, J. I., \& Lee, E. O. (2007). Aquatic exercise in older Korean women with arthritis: identifying barriers to and facilitators of long-term adherence. J.Gerontol.Nurs., 33, 48-56.

Kantor, M. A., Cullinane, E. M., Sady, S. P., Herbert, P. N., \& Thompson, P. D. (1987). Exercise acutely increases high density lipoprotein-cholesterol and lipoprotein lipase activity in trained and untrained men. Metabolism, 36, 188-192.

Kassavou, A., Turner, A., \& French, D. P. (2013). Do interventions to promote walking in groups increase physical activity? A meta-analysis. Int.J.Behav.Nutr.Phys.Act., 10, 18. 
Ke, H. C., Huang, H. J., Liang, K. C., \& Hsieh-Li, H. M. (2011). Selective improvement of cognitive function in adult and aged APP/PS1 transgenic mice by continuous non-shock treadmill exercise. Brain Res., 1403, 1-11.

Kervio, G., Carre, F., \& Ville, N. S. (2003). Reliability and intensity of the six-minute walk test in healthy elderly subjects. Med Sci.Sports Exerc., 35, 169-174.

Kim, S. E., Ko, I. G., Kim, B. K., Shin, M. S., Cho, S., Kim, C. J. et al. (2010). Treadmill exercise prevents aging-induced failure of memory through an increase in neurogenesis and suppression of apoptosis in rat hippocampus. Exp.Gerontol., 45, 357-365.

King, A. C., Haskell, W. L., Young, D. R., Oka, R. K., \& Stefanick, M. L. (1995). Longterm effects of varying intensities and formats of physical activity on participation rates, fitness, and lipoproteins in men and women aged 50 to 65 years. Circulation, 91, 2596-2604.

King, N. A., Caudwell, P., Hopkins, M., Byrne, N. M., Colley, R., Hills, A. P. et al. (2007). Metabolic and behavioral compensatory responses to exercise interventions: barriers to weight loss. Obesity.(Silver.Spring), 15, 1373-1383.

Klusmann, V., Evers, A., Schwarzer, R., Schlattmann, P., Reischies, F. M., Heuser, I. et al. (2010). Complex mental and physical activity in older women and cognitive performance: a 6-month randomized controlled trial. J.Gerontol.A Biol.Sci.Med.Sci., 65, 680-688.

Knowles M.S., Holton III, E. F., \& Swanson RA (2011). The adult learner: The definitive classic in adult education and human resource deveopment. Butterworth-Heinemann. 
Kolb, B. W., I (1990). Fundmentals of Human Neuropsychology. New York: W.H. Freeman and Co.

Komulainen, P. M. K. T. A. L. K. S. M. H. V. K. T. H. R. R. (2010). Exercise, fitness and cognition - a randomised controlled trial in older individuals: the DR's EXTRA study. European Geriatric Medicine, 1, 266-272.

Komulainen, P., Pedersen, M., Hanninen, T., Bruunsgaard, H., Lakka, T. A., Kivipelto, M. et al. (2008). BDNF is a novel marker of cognitive function in ageing women: the DR's EXTRA Study. Neurobiol.Learn.Mem., 90, 596-603.

Konczak, J., Meeuwsen, H. J., \& Cress, M. E. (1992). Changing affordances in stair climbing: the perception of maximum climbability in young and older adults. $J$ Exp.Psychol.Hum.Percept.Perform., 18, 691-697.

Kramer, A. F., Colcombe, S., Erickson, K., Belopolsky, A., McAuley, E., Cohen, N. J. et al. (2002). Effects of aerobic fitness training on human cortical function: a proposal. J.Mol.Neurosci., 19, 227-231.

Kramer, A. F. \& Erickson, K. I. (2007). Capitalizing on cortical plasticity: influence of physical activity on cognition and brain function. Trends Cogn Sci., 11, 342-348.

Kruel, L. L. T. C. A. F. M. R. P. (2009). Effects of Hydrostatic Weight on Heart Rate During Water Immersion. International Journal of Aquatic Research and Education, 3, 178-185. 
Kwak, L., Kremers, S. P., Bergman, P., Ruiz, J. R., Rizzo, N. S., \& Sjostrom, M. (2009). Associations between physical activity, fitness, and academic achievement. J.Pediatr., 155, 914918.

Lachman ME, S. D. N. R. B. a. A. M. J. (2006). The Effects of Strength Training on Memory in Older Adults. Journal of Aging \& Physical Activity, 14, 59-73.

Lajoie, Y., Teasdale, N., Bard, C., \& Fleury, M. (1993). Attentional demands for static and dynamic equilibrium. Exp.Brain Res., 97, 139-144.

Lajoie, Y., Teasdale, N., Bard, C., \& Fleury, M. (1996). Upright standing and gait: are there changes in attentional requirements related to normal aging? Exp.Aging Res., 22, 185-198.

Lautenschlager, N. T., Cox, K. L., Flicker, L., Foster, J. K., van Bockxmeer, F. M., Xiao, J. et al. (2008). Effect of physical activity on cognitive function in older adults at risk for Alzheimer disease: a randomized trial. JAMA, 300, 1027-1037.

Leal, S. L. \& Yassa, M. A. (2013). Perturbations of neural circuitry in aging, mild cognitive impairment, and Alzheimer's disease. Ageing Res.Rev., 12, 823-831.

Lesne, S. \& Kotilinek, L. (2005). Amyloid plaques and amyloid-beta oligomers: an ongoing debate. J.Neurosci., 25, 9319-9320.

Liu-Ambrose, T., Donaldson, M. G., Ahamed, Y., Graf, P., Cook, W. L., Close, J. et al. (2008). Otago home-based strength and balance retraining improves executive functioning in older fallers: a randomized controlled trial. J.Am.Geriatr.Soc., 56, 1821-1830. 
Liu-Ambrose, T., Nagamatsu, L. S., Graf, P., Beattie, B. L., Ashe, M. C., \& Handy, T. C. (2010). Resistance training and executive functions: a 12-month randomized controlled trial. Arch.Intern.Med., 170, 170-178.

Lloyd-Jones, D., Adams, R. J., Brown, T. M., Carnethon, M., Dai, S., De Simone, G. et al. (2010). Heart Disease and Stroke StatisticsGÇö2010 Update: A Report From the American Heart Association. Circulation, 121, e46-e215.

Logsdon, R. G., McCurry, S. M., Pike, K. C., \& Teri, L. (2009). Making physical activity accessible to older adults with memory loss: a feasibility study. Gerontologist, 49 Suppl 1, S94S99.

Lorge, I. (1947). Effective Methods in Adult Education: Report of the Southern Regional Workshop for Agricultural Extension Specialists Raleigh, NC: North Carolina State College.

Luchsinger, J. A., Tang, M. X., Stern, Y., Shea, S., \& Mayeux, R. (2001). Diabetes mellitus and risk of Alzheimer's disease and dementia with stroke in a multiethnic cohort. Am.J.Epidemiol., 154, 635-641.

Madden, D. J., Spaniol, J., Costello, M. C., Bucur, B., White, L. E., Cabeza, R. et al. (2009). Cerebral white matter integrity mediates adult age differences in cognitive performance. J.Cogn Neurosci., 21, 289-302.

Madsen, K., Pedersen, P. K., Djurhuus, M. S., \& Klitgaard, N. A. (1993). Effects of detraining on endurance capacity and metabolic changes during prolonged exhaustive exercise. $J$ Appl.Physiol (1985.), 75, 1444-1451. 
Magistretti, P. J., Sorg, O., Naichen, Y., Pellerin, L., de, R. S., \& Martin, J. L. (1994). Regulation of astrocyte energy metabolism by neurotransmitters. Ren Physiol Biochem., 17, 168171.

Man, D. W., Tsang, W. W., \& Hui-Chan, C. W. (2010). Do older t'ai chi practitioners have better attention and memory function? J.Altern.Complement Med., 16, 1259-1264.

Marcus, B. H., Rossi, J. S., Selby, V. C., Niaura, R. S., \& Abrams, D. B. (1992). The stages and processes of exercise adoption and maintenance in a worksite sample. Health Psychol., 11, 386-395.

Marcus, B. H., Selby, V. C., Niaura, R. S., \& Rossi, J. S. (1992). Self-efficacy and the stages of exercise behavior change. Res.Q.Exerc.Sport, 63, 60-66.

Markowska, A. L., Mooney, M., \& Sonntag, W. E. (1998). Insulin-like growth factor-1 ameliorates age-related behavioral deficits. Neuroscience, 87, 559-569.

Marks, R., Allegrante, J. P., \& Lorig, K. (2005). A review and synthesis of research evidence for self-efficacy-enhancing interventions for reducing chronic disability: implications for health education practice (part II). Health Promot.Pract., 6, 148-156.

Martins, I. J., Hone, E., Foster, J. K., Sunram-Lea, S. I., Gnjec, A., Fuller, S. J. et al. (2006). Apolipoprotein E, cholesterol metabolism, diabetes, and the convergence of risk factors for Alzheimer's disease and cardiovascular disease. Mol.Psychiatry, 11, 721-736. 
Masel, M. C., Raji, M., \& Peek, M. K. (2010). Education and physical activity mediate the relationship between ethnicity and cognitive function in late middle-aged adults. Ethn.Health, $15,283-302$.

McAuley, E., Blissmer, B., Katula, J., Duncan, T. E., \& Mihalko, S. L. (2000). Physical activity, self-esteem, and self-efficacy relationships in older adults: a randomized controlled trial. Ann.Behav.Med, 22, 131-139.

McAuley, E., Jerome, G. J., Elavsky, S., Marquez, D. X., \& Ramsey, S. N. (2003). Predicting long-term maintenance of physical activity in older adults. Prev.Med., 37, 110-118.

McAuley, E., Lox, C., \& Duncan, T. E. (1993). Long-term maintenance of exercise, selfefficacy, and physiological change in older adults. J Gerontol., 48, 218-224.

McAuley, E., Szabo, A. N., Mailey, E. L., Erickson, K. I., Voss, M., White, S. M. et al. (2011). Non-Exercise Estimated Cardiorespiratory Fitness: Associations with Brain Structure, Cognition, and Memory Complaints in Older Adults. Ment.Health Phys.Act., 4, 5-11.

McCartney, N., Hicks, A. L., Martin, J., \& Webber, C. E. (1995). Long-term resistance training in the elderly: effects on dynamic strength, exercise capacity, muscle, and bone. $J$ Gerontol.A Biol.Sci.Med Sci., 50, B97-104.

Meredith-Jones, K., Waters, D., Legge, M., \& Jones, L. (2011). Upright water-based exercise to improve cardiovascular and metabolic health: a qualitative review. Complement Ther.Med, 19, 93-103. 
Michael, J. (2003). Science and Human Behavior: a tutorial in behavior analysis. J.Exp.Anal.Behav., 80, 321-328.

Middleton, L. E., Barnes, D. E., Lui, L. Y., \& Yaffe, K. (2010). Physical activity over the life course and its association with cognitive performance and impairment in old age. J.Am.Geriatr.Soc., 58, 1322-1326.

Middleton, L. E., Manini, T. M., Simonsick, E. M., Harris, T. B., Barnes, D. E., Tylavsky, F. et al. (2011). Activity energy expenditure and incident cognitive impairment in older adults. Arch.Intern.Med., 171, 1251-1257.

Mourot, L., Teffaha, D., Bouhaddi, M., Ounissi, F., Vernochet, P., Dugue, B. et al. (2010). Exercise rehabilitation restores physiological cardiovascular responses to short-term head-out water immersion in patients with chronic heart failure. J.Cardiopulm.Rehabil.Prev., 30, 22-27.

Mueller, A., Kiesewetter, F., Binder, H., Beckmann, M. W., \& Dittrich, R. (2007). Longterm administration of testosterone undecanoate every 3 months for testosterone supplementation in female-to-male transsexuals. J.Clin.Endocrinol.Metab, 92, 3470-3475.

Mullen, S. P., Wojcicki, T. R., Mailey, E. L., Szabo, A. N., Gothe, N. P., Olson, E. A. et al. (2013). A profile for predicting attrition from exercise in older adults. Prev.Sci., 14, 489-496.

Munguia-Izquierdo, D. \& Legaz-Arrese, A. (2008). Assessment of the effects of aquatic therapy on global symptomatology in patients with fibromyalgia syndrome: a randomized controlled trial. Arch.Phys.Med.Rehabil., 89, 2250-2257. 
Myers, J. S. (2008). Factors associated with changing cognitive function in older adults: implications for nursing rehabilitation. Rehabil.Nurs., 33, 117-123.

Myerson J, Emery L, White DA, \& Hale S (2003). Effects of age, domain, and processing demands on memory span: Evidence for differential decline. Aging, Neuropsychology, \& Cognition, 10, 20-27.

Nair, K. S. (1995). Muscle protein turnover: methodological issues and the effect of aging. J Gerontol.A Biol.Sci.Med Sci., 50 Spec No, 107-112.

Nation, D. A., Hong, S., Jak, A. J., Delano-Wood, L., Mills, P. J., Bondi, M. W. et al. (2011). Stress, exercise, and Alzheimer's disease: a neurovascular pathway. Med.Hypotheses, 76, $847-854$.

National Center for Health Statistics (2012). Health, United States, 2011: With Special Feature on Socioeconomic Status and Health Hayattsville, MD.

Nelson, M. E., Rejeski, W. J., Blair, S. N., Duncan, P. W., Judge, J. O., King, A. C. et al. (2007). Physical activity and public health in older adults: recommendation from the American College of Sports Medicine and the American Heart Association. Circulation, 116, 1094-1105.

Nichol, K. E., Parachikova, A. I., \& Cotman, C. W. (2007). Three weeks of running wheel exposure improves cognitive performance in the aged Tg2576 mouse. Behav.Brain Res., $184,124-132$. 
Novak V, P. Z. B. M. E. S. D. A. A. A. P. K. R. M. M. a. P. N. (2011). Adhesion Molecules, Altered Vasoreactivity, and Brain Atrophy in Type 2 Diabetes. Diabetes Care, 34, 2438-2441.

O'Brien Cousins, S. (2005). Overcoming Ageism in Active Living.

O'Brien, S. J. \& Vertinsky, P. A. (1991). Unfit survivors: exercise as a resource for aging women. Gerontologist, 31, 347-357.

O'Neill, K. \& Reid, G. (1991). Perceived barriers to physical activity by older adults. Can.J.Public Health, 82, 392-396.

O'Rourke, H. M. \& Ceci, C. (2013). Reexamining the boundaries of the 'normal' in ageing. Nurs.Inq., 20, 51-59.

Owen, A. M., Downes, J. J., Sahakian, B. J., Polkey, C. E., \& Robbins, T. W. (1990). Planning and spatial working memory following frontal lobe lesions in man. Neuropsychologia, 28, 1021-1034.

Owen, A. M., Roberts, A. C., Polkey, C. E., Sahakian, B. J., \& Robbins, T. W. (1991). Extra-dimensional versus intra-dimensional set shifting performance following frontal lobe excisions, temporal lobe excisions or amygdalo-hippocampectomy in man. Neuropsychologia, 29, 993-1006.

Park, C. H., Chodzko-Zajko, W., Ory, M. G., Gleason-Senior, J., Bazzarre, T. L., \& Mockenhaupt, R. (2010). The impact of a national strategy to increase physical activity among older adults on national organizations. J.Aging Phys.Act., 18, 425-438. 
Park, D. C. \& Reuter-Lorenz, P. (2009). The adaptive brain: aging and neurocognitive scaffolding. Annu.Rev.Psychol., 60, 173-196.

Patton, M. Q. (1999). Enhancing the quality and credibility of qualitative analysis. Health Serv.Res., 34, 1189-1208.

Patton, M. Q. (2002). Qualitative Research \& Evaluation Methods. (3rd ed.) Thousand Oaks: Sage Publications.

Pellerin, L., Pellegri, G., Bittar, P. G., Charnay, Y., Bouras, C., Martin, J. L. et al. (1998). Evidence supporting the existence of an activity-dependent astrocyte-neuron lactate shuttle. Dev.Neurosci., 20, 291-299.

Pereira, A. C., Huddleston, D. E., Brickman, A. M., Sosunov, A. A., Hen, R., McKhann, G. M. et al. (2007). An in vivo correlate of exercise-induced neurogenesis in the adult dentate gyrus. Proc.Natl.Acad.Sci.U.S.A, 104, 5638-5643.

Perrig-Chiello, P., Perrig, W. J., Ehrsam, R., Staehelin, H. B., \& Krings, F. (1998). The effects of resistance training on well-being and memory in elderly volunteers. Age Ageing, 27, $469-475$.

Peters, R. (2006). Ageing and the brain. Postgrad.Med.J., 82, 84-88.

Phillips, E. M., Schneider, J. C., \& Mercer, G. R. (2004). Motivating elders to initiate and maintain exercise. Arch.Phys.Med.Rehabil., 85, S52-S57. 
Pontifex, M. B., Hillman, C. H., Fernhall, B., Thompson, K. M., \& Valentini, T. A. (2009). The effect of acute aerobic and resistance exercise on working memory. Med.Sci.Sports Exerc., 41, 927-934.

Poole, J. L. (1991). Application of motor learning principles in occupational therapy. Am.J Occup.Ther., 45, 531-537.

Poon, L., W.Chodzeko-Zajko, \& PD.Tomporowski (2006). Active Living, Cognitive Functioning, and Aging. (vols. 1) Champaign, IL: Human Kinetics.

Prochaska, J. O. \& Velicer, W. F. (1997). The transtheoretical model of health behavior change. Am.J Health Promot., 12, 38-48.

Radaelli, R., Botton, C. E., Wilhelm, E. N., Bottaro, M., Lacerda, F., Gaya, A. et al. (2013). Low- and high-volume strength training induces similar neuromuscular improvements in muscle quality in elderly women. Exp.Gerontol., 48, 710-716.

Radak, Z., Hart, N., Sarga, L., Koltai, E., Atalay, M., Ohno, H. et al. (2010). Exercise plays a preventive role against Alzheimer's disease. J.Alzheimers.Dis., 20, 777-783.

Radak, Z., Kaneko, T., Tahara, S., Nakamoto, H., Pucsok, J., Sasvari, M. et al. (2001). Regular exercise improves cognitive function and decreases oxidative damage in rat brain. Neurochem.Int., 38, 17-23.

Ratcliff, R., Thapar, A., \& McKoon, G. (2006a). Aging and individual differences in rapid two-choice decisions. Psychon.Bull.Rev., 13, 626-635. 
Ratcliff, R., Thapar, A., \& McKoon, G. (2006b). Aging, practice, and perceptual tasks: a diffusion model analysis. Psychol.Aging, 21, 353-371.

Raz N (2004). The ageing Brain: structural changes and their implications for cognitive ageing. In L.B.L.N.R Dixon (Ed.), New Frontiers in cognitive ageing (pp. 115-134). Oxford: Oxford University Press.

Raz, N. \& Rodrigue, K. M. (2006). Differential aging of the brain: patterns, cognitive correlates and modifiers. Neurosci.Biobehav.Rev., 30, 730-748.

Raz, N., Rodrigue, K. M., \& Acker, J. D. (2003). Hypertension and the brain: vulnerability of the prefrontal regions and executive functions. Behav.Neurosci., 117, 1169-1180.

Redelmeier, D. A., Bayoumi, A. M., Goldstein, R. S., \& Guyatt, G. H. (1997). Interpreting small differences in functional status: the Six Minute Walk test in chronic lung disease patients. Am.J Respir.Crit Care Med, 155, 1278-1282.

Resnick, B., Orwig, D., Magaziner, J., \& Wynne, C. (2002). The effect of social support on exercise behavior in older adults. Clin.Nurs.Res., 11, 52-70.

Rhodes, R. E., Martin, A. D., \& Taunton, J. E. (2001). Temporal relationships of selfefficacy and social support as predictors of adherence in a 6-month strength-training program for older women. Percept.Mot.Skills, 93, 693-703.

Rica, R. L., Carneiro, R. M., Serra, A. J., Rodriguez, D., Pontes Junior, F. L., \& Bocalini, D. S. (2013). Effects of water-based exercise in obese older women: impact of short-term follow- 
up study on anthropometric, functional fitness and quality of life parameters.

Geriatr.Gerontol.Int., 13, 209-214.

Richards, M., Hardy, R., \& Wadsworth, M. E. (2003). Does active leisure protect cognition? Evidence from a national birth cohort. Soc.Sci.Med., 56, 785-792.

Rikli R.E. \& Jones C.J. (1998). The Reliability and Validity of a 6-Minute Walk Test as a Measure of Physical Endurance in Older Adults. Journal of Aging and Physical Activity, 6, 363375.

Rimmer, J. H., Riley, B., Wang, E., \& Rauworth, A. (2005). Accessibility of health clubs for people with mobility disabilities and visual impairments. Am.J.Public Health, 95, 2022-2028.

Ritsner, M. S., Blumenkrantz, H., Dubinsky, T., \& Dwolatzky, T. (2006). The detection of neurocognitive decline in schizophrenia using the Mindstreams Computerized Cognitive Test Battery. Schizophr.Res., 82, 39-49.

Robinson, T. E., White, G. L., Jr., \& Houchins, J. C. (2006). Improving communication with older patients: tips from the literature. Fam.Pract.Manag., 13, 73-78.

Rodriguez, D., Silva, V., Prestes, J., Rica, R. L., Serra, A. J., Bocalini, D. S. et al. (2011). Hypotensive response after water-walking and land-walking exercise sessions in healthy trained and untrained women. Int.J Gen.Med, 4, 549-554.

Rosenstock, I. M. (1988). Adoption and maintenance of lifestyle modifications. Am.J.Prev.Med., 4, 349-352. 
Rosenstock, I. M. (1990). The health belief model: explaining health behavior through expectancies. In Health Behavior and Health Education (pp. 39-62). San Francisco: Jossey-Bass PUblishers.

Rosenstock, I. M., Strecher, V. J., \& Becker, M. H. (1988). Social learning theory and the Health Belief Model. Health Educ.Q., 15, 175-183.

Sallis JF, Owen N, \& Fisher EB (2008). Ecological models of health behavior. In Glanz K, Rimer BK, \& Viswanath K (Eds.), Health Behavior and health education: theory, research and practice (4th ed., pp. 465-86). San Fancisco, CA: Jossey-Bass.

Sanders, M. E., Takeshima, N., Rogers, M. E., Colado, J. C., \& Borreani, S. (2013). Impact of the s.w.e.a.T. water-exercise method on activities of daily living for older women. $J$ Sports Sci.Med, 12, 707-715.

Scahill, R. I., Frost, C., Jenkins, R., Whitwell, J. L., Rossor, M. N., \& Fox, N. C. (2003). A longitudinal study of brain volume changes in normal aging using serial registered magnetic resonance imaging. Arch.Neurol., 60, 989-994.

Scherder, E. J., Eggermont, L. H., Geuze, R. H., Vis, J., \& Verkerke, G. J. (2010). Quadriceps strength and executive functions in older women. Am.J.Phys.Med.Rehabil., 89, 458463.

Schutzer, K. A. \& Graves, B. S. (2004). Barriers and motivations to exercise in older adults. Prev.Med., 39, 1056-1061. 
Scullin, M. K., Gordon, B. A., Shelton, J. T., Lee, J. H., Head, D., \& McDaniel, M. A. (2013). Evidence for a detrimental relationship between hypertension history, prospective memory, and prefrontal cortex white matter in cognitively normal older adults. Cogn Affect.Behav.Neurosci., 13, 405-416.

Seefeldt, V., Malina, R. M., \& Clark, M. A. (2002). Factors affecting levels of physical activity in adults. Sports Med., 32, 143-168.

Seidler, R. D. (2007). Older adults can learn to learn new motor skills. Behav.Brain Res., $183,118-122$.

Sheldahl, L. M., Buskirk, E. R., Loomis, J. L., Hodgson, J. L., \& Mendez, J. (1982). Effects of exercise in cool water on body weight loss. Int.J Obes., 6, 29-42.

Shepard, R. (1987). Physical Activity and Aging. (2nd ed.) London: Croom Helm.

Sheppard, L., Senior, J., Park, C. H., Mockenhaupt, R., Chodzko-Zajko, W., \& Bazzarre, T. (2003). The National Blueprint Consensus Conference summary report: strategic priorities for increasing physical activity among adults aged >or=50. Am.J.Prev.Med., 25, 209-213.

Shumway-Cook, A. \& Woollacott, M. (2000). Attentional demands and postural control: the effect of sensory context. J Gerontol.A Biol.Sci.Med Sci., 55, M10-M16.

SJH Biddle, W Brehm, M Verheijdn, \& M Hopman-Rock (2012). Population physical activity behavior change: A review for the European College of Sport Science. European Journal of Sport Science, 12, 367-383.

Skinner BF (1953). Science and Human Behavior. New York: The Macmillan Company. 
Small, B. J., Dixon, R. A., McArdle, J. J., \& Grimm, K. J. (2012). Do changes in lifestyle engagement moderate cognitive decline in normal aging? Evidence from the Victoria Longitudinal Study. Neuropsychology., 26, 144-155.

Small, G. W. (2002). What we need to know about age related memory loss. BMJ, 324, 1502-1505.

Small, G. W., Rabins, P. V., Barry, P. P., Buckholtz, N. S., DeKosky, S. T., Ferris, S. H. et al. (1997). Diagnosis and treatment of Alzheimer disease and related disorders. Consensus statement of the American Association for Geriatric Psychiatry, the Alzheimer's Association, and the American Geriatrics Society. JAMA, 278, 1363-1371.

Small, G. W., Silverman, D. H., Siddarth, P., Ercoli, L. M., Miller, K. J., Lavretsky, H. et al. (2006). Effects of a 14-day healthy longevity lifestyle program on cognition and brain function. Am.J.Geriatr.Psychiatry, 14, 538-545.

Smith, M. A., Ghazizadeh, A., \& Shadmehr, R. (2006). Interacting adaptive processes with different timescales underlie short-term motor learning. PLoS.Biol., 4, e179.

Smith, P. J., Blumenthal, J. A., Hoffman, B. M., Cooper, H., Strauman, T. A., WelshBohmer, K. et al. (2010). Aerobic exercise and neurocognitive performance: a meta-analytic review of randomized controlled trials. Psychosom.Med., 72, 239-252.

Snowden, M., Steinman, L., Mochan, K., Grodstein, F., Prohaska, T. R., Thurman, D. J. et al. (2011). Effect of exercise on cognitive performance in community-dwelling older adults: review of intervention trials and recommendations for public health practice and research. J.Am.Geriatr.Soc., 59, 704-716. 
Sonstroem, R. J. \& Morgan, W. P. (1989). Exercise and self-esteem: rationale and model. Med Sci.Sports Exerc., 21, 329-337.

Stampfer, M. J. (2006). Cardiovascular disease and Alzheimer's disease: common links. J.Intern.Med., 260, 211-223.

Steffens, D., Beckenkamp, P. R., Hancock, M., Paiva, D. N., Alison, J. A., \& MennaBarreto, S. S. (2013). Activity level predicts 6-minute walk distance in healthy older females: an observational study. Physiotherapy., 99, 21-26.

Stroth, S., Hille, K., Spitzer, M., \& Reinhardt, R. (2009). Aerobic endurance exercise benefits memory and affect in young adults. Neuropsychol.Rehabil., 19, 223-243.

Studenski, S., Carlson, M. C., Fillit, H., Greenough, W. T., Kramer, A., \& Rebok, G. W. (2006). From bedside to bench: does mental and physical activity promote cognitive vitality in late life? Sci.Aging Knowledge.Environ., 2006, e21.

Suetterlin, K. J. \& Sayer, A. A. (2013). Proprioception: where are we now? A commentary on clinical assessment, changes across the life course, functional implications and future interventions. Age and ageing, aft174.

Sullivan R.L., W. J. L., Arnold, S. S., \& Sarkees, M. D. (1990). The Trainer's guide: A practical manual for the design, delivery, and evaulation of training. Aspen Publishers.

Suomi, R. \& Koceja, D. M. (2000). Postural sway characteristics in women with lower extremity arthritis before and after an aquatic exercise intervention. Arch.Phys.Med.Rehabil., 81, 780-785. 
Swainson, R., Hodges, J. R., Galton, C. J., Semple, J., Michael, A., Dunn, B. D. et al. (2001). Early detection and differential diagnosis of Alzheimer's disease and depression with neuropsychological tasks. Dement.Geriatr.Cogn Disord., 12, 265-280.

Swank, A. M., Kachelman, J. B., Bibeau, W., Quesada, P. M., Nyland, J., Malkani, A. et al. (2011). Prehabilitation before total knee arthroplasty increases strength and function in older adults with severe osteoarthritis. J Strength Cond.Res., 25, 318-325.

Swardfager, W., Herrmann, N., Marzolini, S., Saleem, M., Kiss, A., Shammi, P. et al. (2010). Cardiopulmonary fitness is associated with cognitive performance in patients with coronary artery disease. J.Am.Geriatr.Soc., 58, 1519-1525.

swisher AK \& Goldfarb AH (1998). Use of the six-minute walk/run test to predict peak oxygen consumption in older adults. Cardiopulmonary Physical Therapy Journal, 9, 3-5.

Taaffe, D. R., Duret, C., Wheeler, S., \& Marcus, R. (1999). Once-weekly resistance exercise improves muscle strength and neuromuscular performance in older adults. $J$ Am.Geriatr.Soc., 47, 1208-1214.

Teixeira, C. V., Gobbi, L. T., Corazza, D. I., Stella, F., Costa, J. L., \& Gobbi, S. (2012). Non-pharmacological interventions on cognitive functions in older people with mild cognitive impairment (MCI). Arch.Gerontol.Geriatr., 54, 175-180.

Terblanche, E. \& Millen, A. M. (2012). The magnitude and duration of post-exercise hypotension after land and water exercises. Eur.J Appl.Physiol, 112, 4111-4118. 
Tidhar, D., Drouin, J., \& Shimony, A. (2007). Aqua lymphatic therapy in managing lower extremity lymphedema. J.Support.Oncol., 5, 179-183.

Tomporowski PD, N. E. (1986). Effects of Exercise on Cognitive Processes: A Review. Psychological Bulletin, 99, 338-346.

Triplett, N. T., Colado, J. C., Benavent, J., Alakhdar, Y., Madera, J., Gonzalez, L. M. et al. (2009). Concentric and impact forces of single-leg jumps in an aquatic environment versus on land. Med.Sci.Sports Exerc., 41, 1790-1796.

Tucker, J. M., Welk, G. J., \& Beyler, N. K. (2011). Physical activity in U.S.: adults compliance with the Physical Activity Guidelines for Americans. Am.J.Prev.Med., 40, 454-461.

Ullrich, C., Pirchl, M., \& Humpel, C. (2010). Hypercholesterolemia in rats impairs the cholinergic system and leads to memory deficits. Mol.Cell Neurosci., 45, 408-417.

Van Boxtel, M. P., Langerak, K., Houx, P. J., \& Jolles, J. (1996). Self-reported physical activity, subjective health, and cognitive performance in older adults. Exp.Aging Res., 22, 363379.

Van Boxtel, M. P., Paas, F. G., Houx, P. J., Adam, J. J., Teeken, J. C., \& Jolles, J. (1997). Aerobic capacity and cognitive performance in a cross-sectional aging study. Med.Sci.Sports Exerc., 29, 1357-1365.

Van der Borght, K., Havekes, R., Bos, T., Eggen, B. J., \& Van der Zee, E. A. (2007). Exercise improves memory acquisition and retrieval in the Y-maze task: relationship with hippocampal neurogenesis. Behav.Neurosci., 121, 324-334. 
van Praag, H. (2009). Exercise and the brain: something to chew on. Trends Neurosci., 32, 283-290.

van Uffelen, J. G., Chinapaw, M. J., Hopman-Rock, M., \& van, M. W. (2009). Feasibility and effectiveness of a walking program for community-dwelling older adults with mild cognitive impairment. J.Aging Phys.Act., 17, 398-415.

Verhey, F. R., Huppert, F. A., Korten, E. C., Houx, P., de, V. M., van, L. N. et al. (2003). Cross-national comparisons of the Cambridge Cognitive Examination-revised: the CAMCOG-R: results from the European Harmonization Project for Instruments in Dementia. Age Ageing, 32, 534-540.

Vidal, J. (2002). Updated review on the benefits of weight loss. Int.J Obes.Relat Metab Disord., 26 Suppl 4, S25-S28.

Voss, M. W., Heo, S., Prakash, R. S., Erickson, K. I., Alves, H., Chaddock, L. et al. (2013). The influence of aerobic fitness on cerebral white matter integrity and cognitive function in older adults: results of a one-year exercise intervention. Hum.Brain Mapp., 34, 2972-2985.

Voss, M. W., Nagamatsu, L. S., Liu-Ambrose, T., \& Kramer, A. F. (2011). Exercise, brain, and cognition across the life span. J.Appl.Physiol, 111, 1505-1513.

Voss, M. W., Prakash, R. S., Erickson, K. I., Basak, C., Chaddock, L., Kim, J. S. et al. (2010). Plasticity of brain networks in a randomized intervention trial of exercise training in older adults. Front Aging Neurosci., 2. 
Walsh, J. M., Pressman, A. R., Cauley, J. A., \& Browner, W. S. (2001). Predictors of physical activity in community-dwelling elderly white women. J.Gen.Intern.Med., 16, 721-727.

Wang, T. J., Belza, B., Elaine, T. F., Whitney, J. D., \& Bennett, K. (2007). Effects of aquatic exercise on flexibility, strength and aerobic fitness in adults with osteoarthritis of the hip or knee. J.Adv.Nurs., 57, 141-152.

Werle, C. O., Wansink, B., \& Payne, C. R. (2011). Just thinking about exercise makes me serve more food. Physical activity and calorie compensation. Appetite, 56, 332-335.

Westerman, M. A., Cooper-Blacketer, D., Mariash, A., Kotilinek, L., Kawarabayashi, T., Younkin, L. H. et al. (2002). The relationship between Abeta and memory in the Tg2576 mouse model of Alzheimer's disease. J.Neurosci., 22, 1858-1867.

Weuve, J., Kang, J. H., Manson, J. E., Breteler, M. M., Ware, J. H., \& Grodstein, F. (2004). Physical activity, including walking, and cognitive function in older women. JAMA, 292, 1454-1461.

Whelton, S. P., Chin, A., Xin, X., \& He, J. (2002). Effect of aerobic exercise on blood pressure: a meta-analysis of randomized, controlled trials. Ann.Intern.Med., 136, 493-503.

White, L. J., Dressendorfer, R. H., Holland, E., McCoy, S. C., \& Ferguson, M. A. (2005). Increased caloric intake soon after exercise in cold water. Int.J Sport Nutr.Exerc.Metab, 15, 3847.

Wiener, J. M., Hanley, R. J., Clark, R., \& Van Nostrand, J. F. (1990). Measuring the activities of daily living: comparisons across national surveys. J.Gerontol., 45, S229-S237. 
Willingham, D. B. (1998). A neuropsychological theory of motor skill learning. Psychol.Rev., 105, 558-584.

Willingham, D. B., Peterson, E. W., Manning, C., \& Brashear, H. R. (1997). Patients with Alzheimer's disease who cannot perform some motor skills show normal learning of other motor skills. Neuropsychology, 11, 261-271.

Wilson, R. S., Barnes, L. L., Krueger, K. R., Hoganson, G., Bienias, J. L., \& Bennett, D. A. (2005). Early and late life cognitive activity and cognitive systems in old age. J.Int.Neuropsychol.Soc., 11, 400-407.

Winter, B., Breitenstein, C., Mooren, F. C., Voelker, K., Fobker, M., Lechtermann, A. et al. (2007). High impact running improves learning. Neurobiol.Learn.Mem., 87, 597-609.

Woollacott, M. \& Shumway-Cook, A. (2002). Attention and the control of posture and gait: a review of an emerging area of research. Gait.Posture., 16, 1-14.

Yaguez, L., Shaw, K. N., Morris, R., \& Matthews, D. (2011). The effects on cognitive functions of a movement-based intervention in patients with Alzheimer's type dementia: a pilot study. Int.J.Geriatr.Psychiatry, 26, 173-181.

Yu, F., Ryan, L. H., Schaie, K. W., Willis, S. L., \& Kolanowski, A. (2009). Factors associated with cognition in adults: the Seattle Longitudinal Study. Res.Nurs.Health, 32, 540550.

Zec, R. F. (1995). The neuropsychology of aging. Exp.Gerontol., 30, 431-442. 
Zimet GD, Dahlem NW, Zimet SG, \& Farley GK (1988). The Multidimensional Scale of Percieved Social Support. The Journal of Personality Assessment, 52, 30-41.

Zizzi, S. (2012). Participant Survey.

Ref Type: Personal Communication

Zlomanczuk, P., Milczarek, B., Dmitruk, K., Sikorski, W., Adamczyk, W., Zegarski, T. et al. (2006). Improvement in the face/name association performance after three months of physical training in elderly women. J.Physiol Pharmacol., 57 Suppl 4, 417-424. 